\title{
SPURENELEMENTUNTERSUCHUNGEN AN BODENGELAGERTEM SKELETTMATERIAL
}

\section{VALIDITÄTSERWÄGUNGEN IM KONTEXT DIAGENETISCH BEDINGTER KONZENTRATIONSÄNDERUNGEN DES KNOCHENMINERALS}

\author{
Dissertation zur Erlangung des Doktorgrades \\ der Mathematisch-Naturwissenschaftlichen Fakultäten \\ der Georg-August-Universität zu Göttingen
}

vorgelegt von

Alexander Fabig

aus Mühlhausen

Göttingen 2002 
D 7

Referent:

Korreferent:

Tag der mündlichen Prüfungen:
Prof. Dr. B. Herrmann

Prof. Dr. H. Rothe

24. April 2002 


\section{INHALTSVERZEICHNIS}

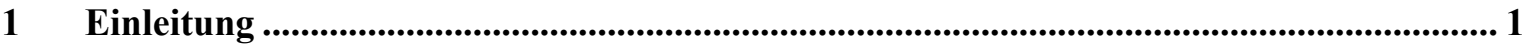

1.1 Ernährungsrekonstruktion durch Interpretation von Spurenelementgehalten in

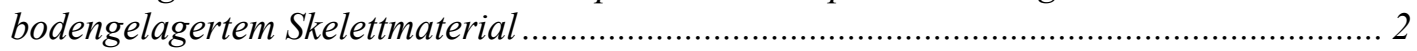

1.2 Die Kontroverse zur Diagenese der Knochensubstanz - ein (v-)erkanntes Problem? ........ 5

2 Konzeption der Untersuchungen ............................................................................................... 11

2.1 Die Möglichkeit diagenetischer Konzentrationsveränderungen: Überprüfungen zur populationsinternen Variabilität von $\mathrm{Ba} / \mathrm{Ca}$ - und $\mathrm{Sr} / \mathrm{Ca}$-Werten ...................................... 11

Histologiegruppen als diagenetische Gruppen ............................................................ 12

Populationsinterne Gegenüberstellung von diagenetischen und ernährungsbedingten

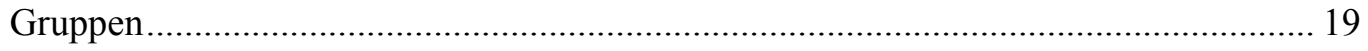

Interpopulationsvergleich diagenetischer Konzentrationsgruppen ............................. 21

2.2 Reversibilität diagenetischer Konzentrationsveränderungen: Untersuchungen zur

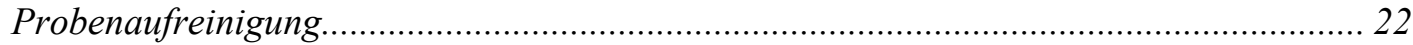

2.3 Das Problem einer zuverlässigen Diagnose diagenetischer Veränderungen: Kontrollkriterien im Test ................................................................................... 26

Der ernährungsbedingte Barium-Strontium-Zusammenhang innerhalb einer

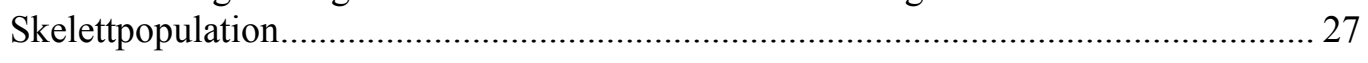

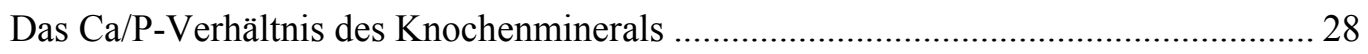

Magnesium (und Natrium) als knochenimmanente Elemente ..................................... 30

Aluminium als kontaminationsanzeigendes Element................................................ 31

Masseverlust der Knochenproben bei der chemischen Probenaufreinigung.................. 31

Masseverlust der Knochenprobe beim Veraschen .................................................. 32

2.4 Diagenetische Veränderungen des Knochenminerals im Detail: Mikroanalytische

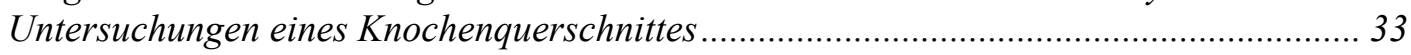

2.5 Ein diageneseunabhängiges Problem: Untersuchung zur intraossären Variabilität von

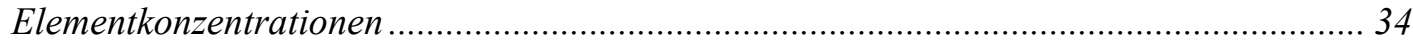

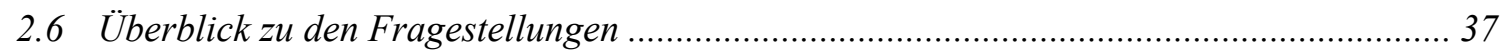

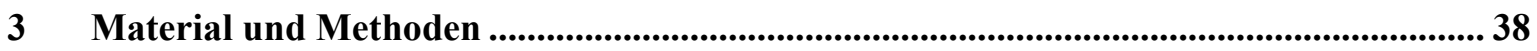

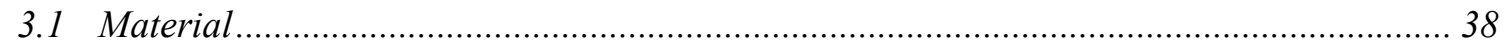

3.1.1 Skelettserien für die Untersuchungen zur populationsinternen Variabilität von

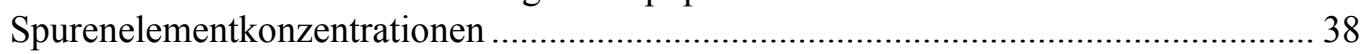

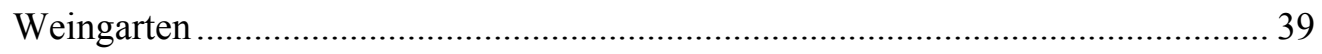

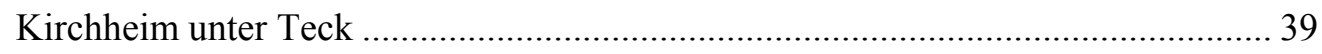

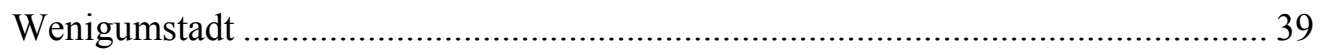

3.1.2 Material für die Untersuchungen zur Probenaufreinigung und für die mikroanalytischen

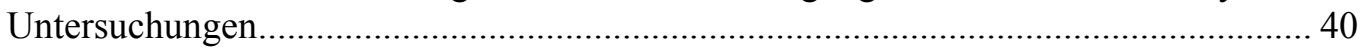

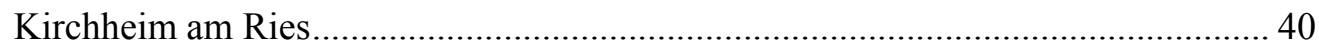

3.1.3 Rezentes Knochenmaterial für die Untersuchung zur intraossären Variabilität von

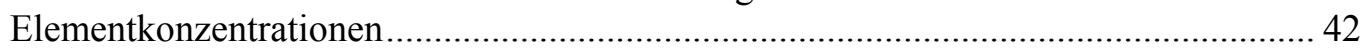

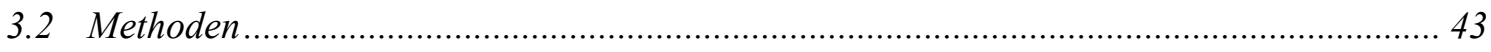

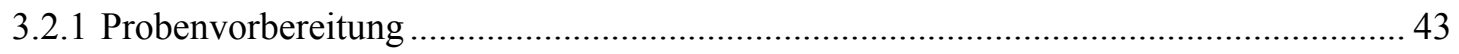

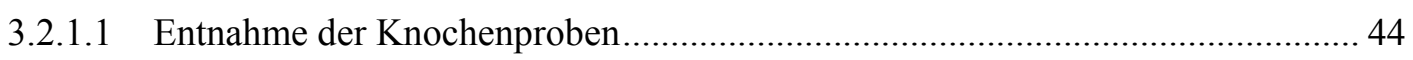

3.2.1.2 Herstellung der histologischen Knochenpräparate............................................. 45

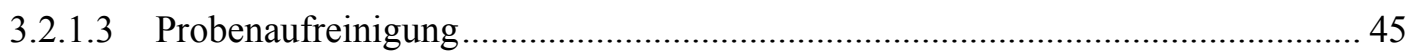


3.2.1.4 Vorbereitung der Proben zur Elementanalytik in Lösung.................................... 47

3.2.1.5 Massekontrollen während Probenaufreinigung und -vorbereitung ....................... 48

3.2.1.6 Modifikationen der Probenvorbereitung für den Untersuchungsteil zur

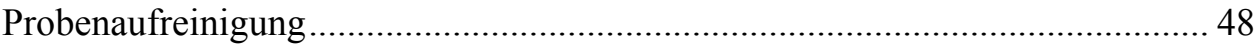

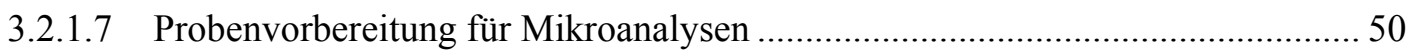

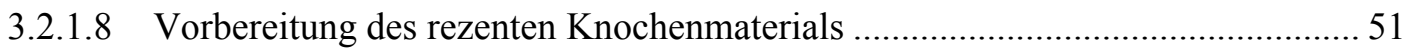

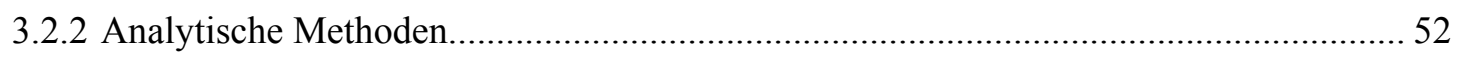

3.2.2.1 Konzentrationsbestimmungen mit AAS, Photometrie und ICP-OES ……........... 52

3.2.2.2 Mikroanalysen mit EPMA und LA-ICP-TOFMS ............................................... 54

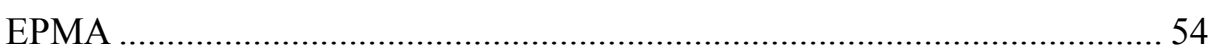

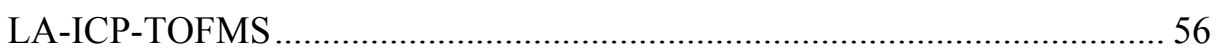

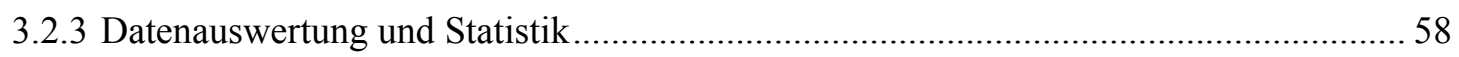

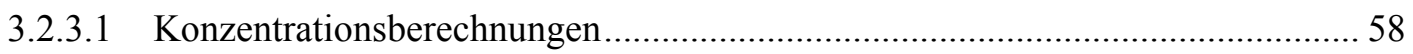

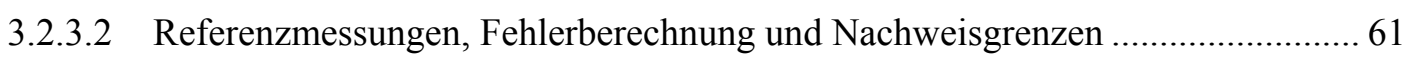

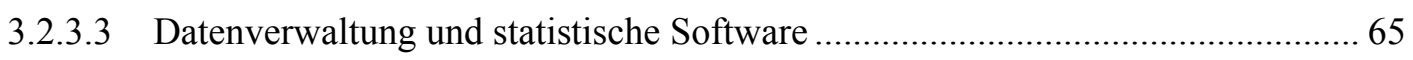

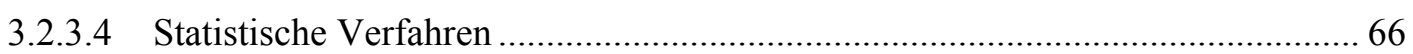

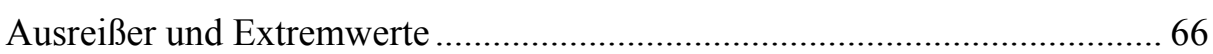

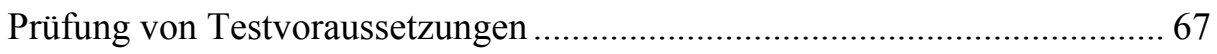

Entscheidung zwischen parametrischen und nichtparametrischen Verfahren zur Prüfung von Unterschiedshypothesen .................................................... 68

Verwendete Verfahren zur Prüfung von Unterschiedshypothesen .................. 69

Verwendetes Verfahren zur Prüfung von Zusammenhangshypothesen ......... 71

Testvorgaben und Signifikanzniveau.......................................................... 72

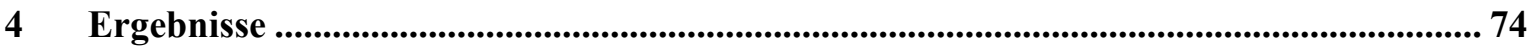

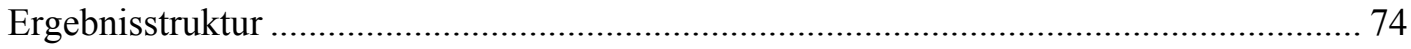

4.1 Ergebnisse zur populationsinternen Variabilität von $\mathrm{Ba} / \mathrm{Ca}$ - und $\mathrm{Sr} / \mathrm{Ca}$-Werten ................ 75

Ein Gruppenmuster der $\mathrm{Ba} / \mathrm{Ca}$ - und $\mathrm{Sr} / \mathrm{Ca}-W e r t e$ in der Skelettpopulation von

Weingarten und die Varianzanalyse seiner möglichen Faktoren ................................... 75

Histologischer Erhaltungszustand und Konzentrationsgruppen in den Populationen von

Kirchheim unter Teck und Wenigumstadt ................................................................. 78

Histologischer Erhaltungszustand und ein systematisches Gruppenmuster................... 80

4.2 Ergebnisse der Untersuchungen zur Probenaufreinigung ........................................... 82

Histologischer Erhaltungszustand und Konzentrationsgruppen in der Population von

Kirchheim am Ries vor und nach der Probenaufreinigung .......................................... 82

Aufreinigungsbedingte Veränderungen der Barium- und Strontiumkonzentrationen im

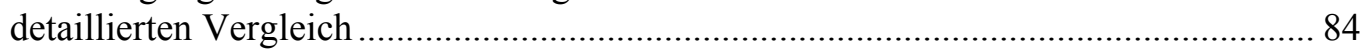

Zusammenfassung der Ergebnisse der Untersuchungen zur Probenaufreinigung ......... 87

4.3 Ergebnisse zur Prüfung diageneseanzeigender Kontrollkriterien .................................. 87

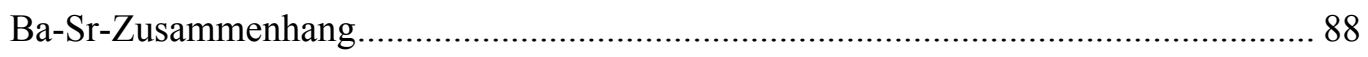

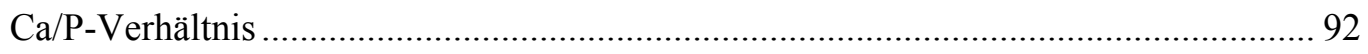

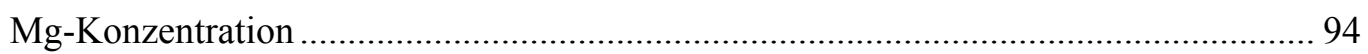

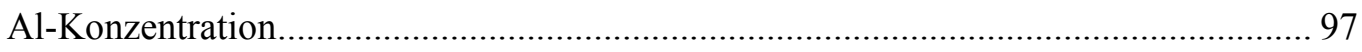

Masseverlust bei der Probenaufreinigung durch Ätzen .............................................. 100

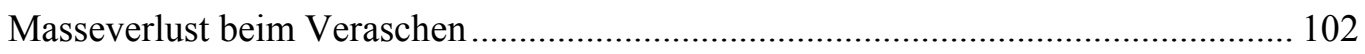

Zusammenfassung der Ergebnisse zu den diagenesecharakterisierenden

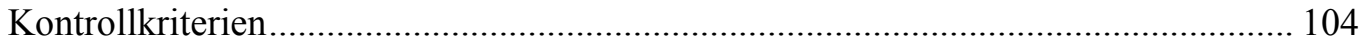


4.4 Ergebnisse der mikroanalytischen Untersuchungen eines Kompaktaquerschnittes ......... 106

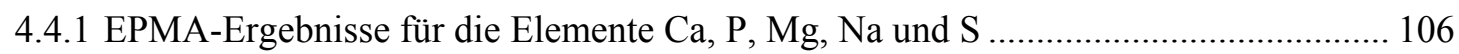

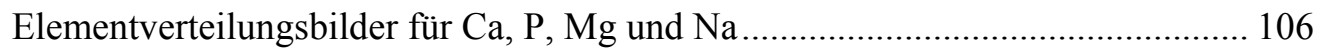

Einzelpunktanalysen der Konzentrationen von $\mathrm{Ca}, \mathrm{P}, \mathrm{Mg}, \mathrm{Na}$ und $\mathrm{S}$..................... 112

4.4.2 LA-ICP-TOFMS-Ergebnisse der Elemente Ba, Sr und U ............................................ 116

4.4.3 Zusammenfassung der mikroanalytischen Ergebnisse................................................ 121

4.4.4 Nachtrag zum Element Na: Vergleich zwischen gut und schlecht erhaltenen

Knochenproben in einer Stichprobe von Kirchheim am Ries ...................................... 123

4.5 Intraossäre Variabilität der Sr- und Mg-Konzentrationen .............................................. 125

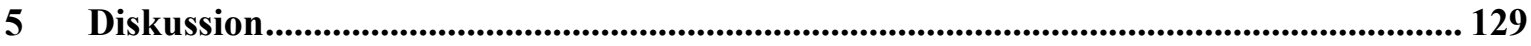

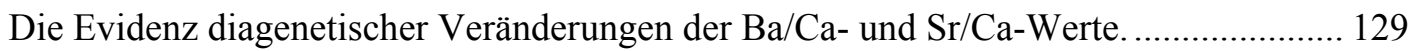

Der systematische Charakter der diagenetischen Veränderungen der $\mathrm{Ba} / \mathrm{Ca}-$ und $\mathrm{Sr} / \mathrm{Ca}-$

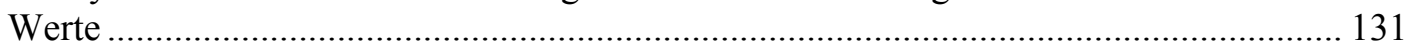

Probenaufreinigung und diageneseanzeigende Kontrollkriterien - Möglichkeiten einer erweiterten Charakterisierung der Knochendiagenese ...................................................... 135

Schlußfolgerungen für die Interpretationsmöglichkeiten von

Spurenelementkonzentrationen in bodengelagertem Skelettmaterial.

Die intraossäre Konzentrationsvariabilität: Elementverteilung im Knochenmineral undifferenziert oder knochenphysiologisch systematisch? ........................................... 142

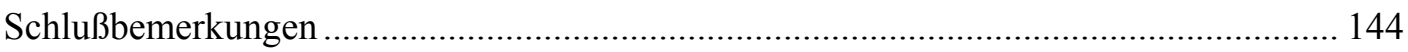

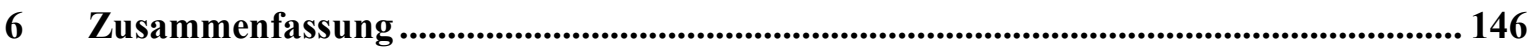

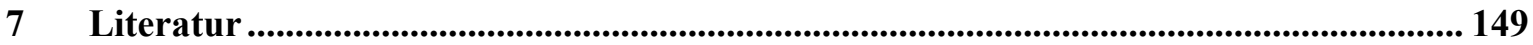

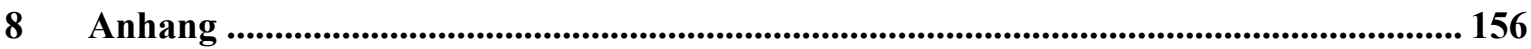

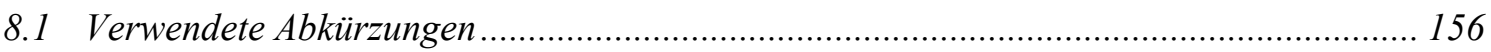

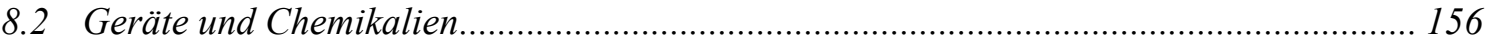

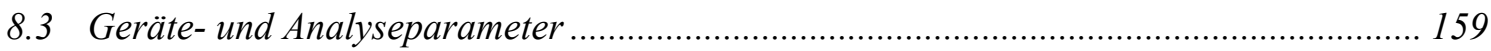

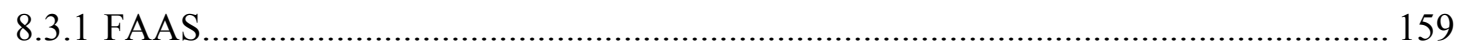

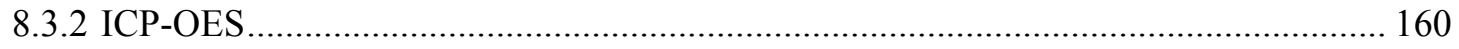

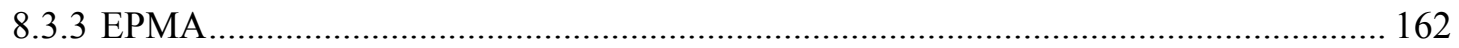

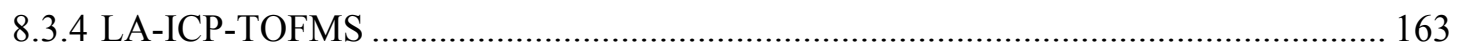

8.4 Konzentrationsberechnungen, Referenzmessungen, Meßfehler und Nachweisgrenzen .... 163

8.5 Probenlisten, Meßdaten und tabellierte statistisch deskriptive Daten .............................. 166 


\section{ABBILDUNGSVERZEICHNIS}

Abbildung 1: Lichtmikroskopische Aufnahmen eines Knochendünnschnittes von einer histologisch als gut kategorisierten Knochenprobe von Kirchheim am Ries (KR 106). ............................. 16

Abbildung 2: Lichtmikroskopische Aufnahmen eines Knochendünnschnittes von einer histologisch als mittel kategorisierten Knochenprobe von Kirchheim am Ries (KR 261), ....................... 17

Abbildung 3: Lichtmikroskopische Aufnahmen eines Knochendünnschnittes von einer histologisch als schlecht kategorisierten Knochenprobe von Kirchheim am Ries (KR 206).

Abbildung 4: Lageplan der Bestattungen des Gräberfeldes von Kirchheim am Ries mit markierten Grabstellen der untersuchten Knochenproben kategorisiert nach histologischem Erhaltungszustand.

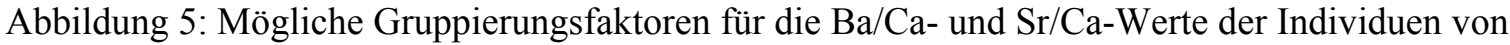
Weingarten.

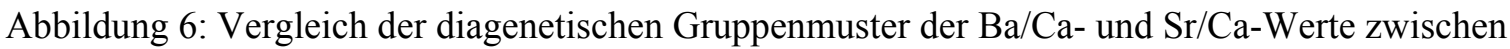
a) Kirchheim unter Teck, b) Wenigumstadt und c) Weingarten.

Abbildung 7: Statistisch deskriptiver Vergleich der Ba/Ca- (a-c) und $\mathrm{Sr} / \mathrm{Ca}-W e r t e(d-f)$ zwischen den Histologiegruppen für Kirchheim unter Teck $(a+d)$, Wenigumstadt $(b+e)$ und Weingarten $(\mathrm{c}+\mathrm{f})$.

Abbildung 8: Das Gruppenmuster (gute vs. schlechte Histologie) der $\mathrm{Ba} / \mathrm{Ca}-$ und $\mathrm{Sr} / \mathrm{Ca}-\mathrm{Werte}$ von

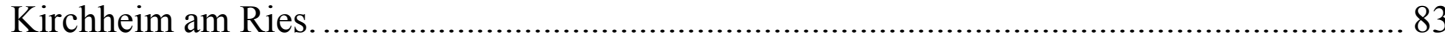

Abbildung 9: Statistisch deskriptiver Vergleich von a) Ba/Ca-Werten und b) $\mathrm{Sr} / \mathrm{Ca}-W e r t e n$ zwischen den Histologiegruppen von Kirchheim am Ries.

Abbildung 10: Aufreinigungsbedingte Veränderungen der $\mathrm{Ba} / \mathrm{Ca}-\mathrm{Werte}$ (a, c, e) und $\mathrm{Sr} / \mathrm{Ca}-\mathrm{Werte}$ $(b, d, f)$ für die Knochenproben von Kirchheim am Ries.

Abbildung 11: Vergleich der gruppeninternen Zusammenhänge von $\mathrm{Ba} / \mathrm{Ca}-$ und $\mathrm{Sr} / \mathrm{Ca}-W e r t e n$ zwischen den Histologiegruppen für Kirchheim unter Teck $(a+d)$, Wenigumstadt $(b+e)$ und Weingarten $(\mathrm{c}+\mathrm{f})$.

Abbildung 12: Vergleich der gruppeninternen Zusammenhänge von $\mathrm{Ba} / \mathrm{Ca}-$ und $\mathrm{Sr} / \mathrm{Ca}-W e r t e n$ zwischen den Histologiegruppen für Kirchheim am Ries.

Abbildung 13: Statistisch deskriptiver Vergleich der Ca/P-Werte zwischen den Histologiegruppen für a) Kirchheim unter Teck, b) Wenigumstadt, c) Weingarten und d) Kirchheim am Ries... 93

Abbildung 14: Statistisch deskriptiver Vergleich der Mg/Ca-Werte zwischen den Histologiegruppen von Kirchheim am Ries.

Abbildung 15: Aufreinigungsbedingte Veränderungen der $\mathrm{Mg} / \mathrm{Ca}-W e r t e$ für die Knochenproben von Kirchheim am Ries.

Abbildung 16: Vergleich der Al/Ca-Werte zwischen den Histologiegruppen von Kirchheim am Ries vor und nach verschiedenen Methoden der Probenaufreinigung.

Abbildung 17: Aufreinigungsbedingte Veränderungen der $\mathrm{Al} / \mathrm{Ca}-W e r t e$ für die Knochenproben von Kirchheim am Ries.

Abbildung 18: Statistisch deskriptiver Vergleich der durch das Ätzen bedingten Masseverluste zwischen den Histologiegruppen.....

Abbildung 19: Statistisch deskriptiver Vergleich der durch das Veraschen bedingten Masseverluste zwischen den Histologiegruppen für a) Wenigumstadt, b) Weingarten und c) Kirchheim am Ries.

Abbildung 20: Ca- und P-Intensitäten (counts per step, als Maß für die jeweiligen Elementkonzentrationen) der Elementverteilungsanalyse mit der EPMA für den periostnahen Bereich des Kompaktaquerschnittes der Knochenprobe KR 261 von Kirchheim am Ries... 107

Abbildung 21: Lichtmikroskopische Aufnahmen eines Dünnschnittes (Transversalebene) der Probe KR 261 von Kirchheim am Ries. 
Abbildung 22: Elementverteilungen im periostnahen Bereich des Kompaktaquerschnittes von KR 261

Abbildung 23: Elementverteilungen im markraumnahen Bereich des Kompaktaquerschnittes von KR 261.

Abbildung 24: SEM-Detailaufnahmen im Composition-Modus vom Kompaktaquerschnitt der Knochenprobe KR 261.

Abbildung 25: Ca- und P-Gehalte und resultierende $\mathrm{Ca} / \mathrm{P}-W e r t e$ (eingefügte Grafik) der EPMAMeßpunkte im Kompaktaquerschnitt.

Abbildung 26: $\mathrm{Na} / \mathrm{Ca}-, \mathrm{Mg} / \mathrm{Ca}$ - und $\mathrm{S} / \mathrm{Ca}-W e r t e$ sowie die Mineralsummen innerhalb eines Kompaktaquerschnittes mittels EMP bestimmt und nach knochenmineralisch "nativen" bzw. diagenetisch veränderten Mineralarealen kategorisiert.

Abbildung 27: Auswertung der Meßpunkte der LA-ICP-TOFMS-Analyse.

Abbildung 28: Korrelation der $\mathrm{Ba} / \mathrm{Ca}$ - und $\mathrm{Sr} / \mathrm{Ca}$-Signalquotienten der LA-ICP-TOFMS-Analysen innerhalb des Kompaktaquerschnittes der Knochenprobe KR 261 von Kirchheim am Ries. 121

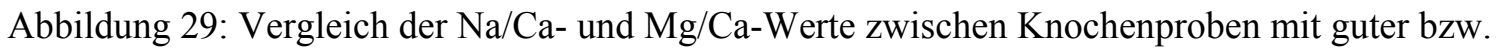
schlechter histologischer Erhaltung von Kirchheim am Ries.

Abbildung 30: Sr/Ca-Werte-Verteilung innerhalb einer Femurdiaphyse (rechts, männlich, senil).

Abbildung 31: Mg/Ca-Werte-Verteilung innerhalb einer Femurdiaphyse (rechts, männlich, senil). 


\section{TABELLENVERZEICHNIS}

Tabelle 1: Ergebnisse für die drei möglichen Gruppierungsfaktoren der $\mathrm{Ba} / \mathrm{Ca}-$ und $\mathrm{Sr} / \mathrm{Ca}-\mathrm{Werte}$ von Weingarten (nichtparametrische Varianzanalyse).

Tabelle 2: Statistische Vergleiche (Mann-Whitney-U-Test) der histologischen Gruppen von Kirchheim unter Teck und Wenigumstadt hinsichtlich Unterschieden der $\mathrm{Ba} / \mathrm{Ca}$ - und $\mathrm{Sr} / \mathrm{Ca}-$ Werte.

Tabelle 3: Statistische Überprüfungen für die $\mathrm{Ba} / \mathrm{Ca}$ - und $\mathrm{Sr} / \mathrm{Ca}$-Werte von Kirchheim am Ries. . 85

Tabelle 4: Korrelationsanalysen (Produkt-Moment-Korrelation) für den Zusammenhang der $\mathrm{Ba} / \mathrm{Ca}$ und der $\mathrm{Sr} / \mathrm{Ca}-W e r t e$.

Tabelle 5: Vergleiche der Histologiegruppen hinsichtlich der Ca/P-Werte (Mann-Whitney-U-Test)

Tabelle 6: Statistische Überprüfungen für die $\mathrm{Mg} / \mathrm{Ca}$-Werte von Kirchheim am Ries..................... 96

Tabelle 7: Statistische Überprüfungen für die Al/Ca-Werte von Kirchheim am Ries...................... 99

Tabelle 8: Vergleiche der Histologiegruppen hinsichtlich Masseverlust durch Ätzen (MannWhitney-U-Test)

Tabelle 9: Vergleiche der Histologiegruppen hinsichtlich Veraschungsverlusten (Mann-Whitney-UTest).

Tabelle 10: Vergleich "nativer" und veränderter Mineralareale innerhalb eines

Kompaktaquerschnittes bezüglich der $\mathrm{Ca} / \mathrm{P}-, \mathrm{Na} / \mathrm{Ca}-, \mathrm{Mg} / \mathrm{Ca}$ - und $\mathrm{S} / \mathrm{Ca}-W e r t e$ anhand der

EPMA-Einzelmeßpunkte (Mann-Whitney-U-Test) .

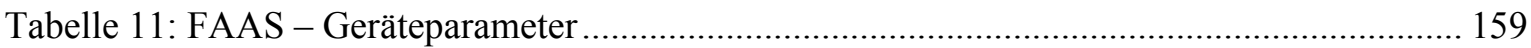

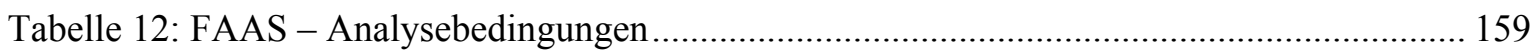

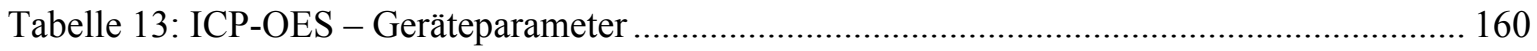

Tabelle 14: ICP-OES - Elementspezifische Analyseparameter....................................................... 161

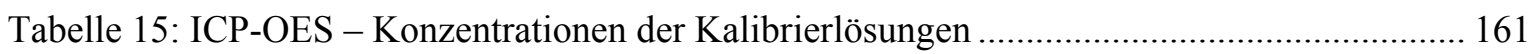

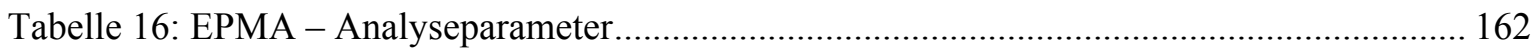

Tabelle 17: EPMA - Kalibrierstandards und elementspezifische Analyseparameter..................... 162

Tabelle 18: LA-ICP-TOFMS - Geräte- und Analyseparameter ................................................... 163

Tabelle 19: EPMA und ICP-OES - Faktoren für die Konzentrationsumrechnung

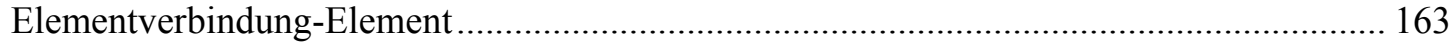

Tabelle 20: Konzentrationen der Referenzmaterialien SRM 1400 und Animal Bone H5 ............. 164

Tabelle 21: FAAS - Ergebnisse der Referenzmessungen und resultierende relative Fehler für die Konzentrationsquotienten.

Tabelle 22: ICP-OES - Ergebnisse der Referenzmessungen des SRM 1400 und die resultierenden relativen Fehler für die Konzentrationsquotienten ............................................................ 165

Tabelle 23: ICP-OES - Nachweisgrenzen für die Elemente in der Meßlösung und deren Umrechnung auf Nachweisgrenze als Knochenkonzentrationen

Tabelle 24: EPMA - Nachweisgrenzen und relative Fehler der auswertungsrelevanten Elemente einschließlich der resultierenden relativen Fehler für die Konzentrationsquotienten. .......... 165

Tabelle 25: Weingarten - Probenliste und auswertungsrelevante Individualdaten......................... 166

Tabelle 26: Kirchheim unter Teck - Probenliste und auswertungsrelevante Individualdaten....... 168

Tabelle 27: Wenigumstadt - Probenliste und auswertungsrelevante Individualdaten.................. 170

Tabelle 28: Kirchheim am Ries - Auswertungsrelevante Individualdaten für den unbehandelten Probensatz.

Tabelle 29: Kirchheim am Ries - Auswertungsrelevante Individualdaten für den Probensatz, der nur geätzt wurde. 
Tabelle 30: Kirchheim am Ries - Auswertungsrelevante Individualdaten für den Probensatz, der oberflächenbehandelt wurde.

Tabelle 31: Kirchheim am Ries - Auswertungsrelevante Individualdaten für den Probensatz, der oberflächenbehandelt und geätzt wurde.

Tabelle 32: Kirchheim am Ries - Daten von den Natriumanalysen .......................................... 175

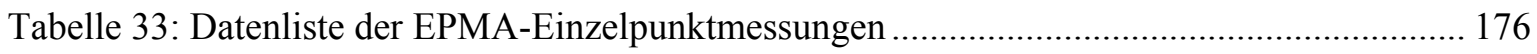

Tabelle 34: Datenliste der LA-ICP-TOFMS Analysen - Meßsignale und Signalquotienten. ....... 177

Tabelle 35: Rezente Femurdiaphyse - Liste der Einzelproben einschließlich auswertungsrelevanter

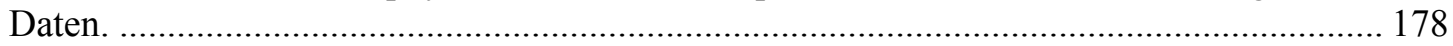

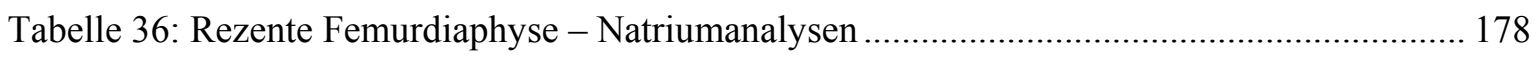

Tabelle 37: Weingarten - Statistisch deskriptive Daten für die drei Gruppierungsfaktoren.......... 179

Tabelle 38: Kirchheim unter Teck - Statistisch deskriptive Daten der Histologiegruppen ........... 179

Tabelle 39: Wenigumstadt - Statistisch deskriptive Daten der Histologiegruppen ....................... 179

Tabelle 40: Kirchheim am Ries - Statistisch deskriptive Daten der Histologiegruppen des

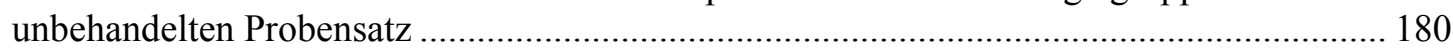

Tabelle 41: Kirchheim am Ries - Statistisch deskriptive Daten der Histologiegruppen des geätzten Probensatzes ....

Tabelle 42: Kirchheim am Ries - Statistisch deskriptive Daten der Histologiegruppen des oberflächenbehandelten Probensatz

Tabelle 43: Kirchheim am Ries - Statistisch deskriptive Daten der Histologiegruppen des Probensatzes, der oberflächenbehandelt und geätzt wurde

Tabelle 44: EPMA-Analysen - Statistisch deskriptive Daten für "native" und veränderte Mineralareale innerhalb des Kompaktaquerschnittes der Knochenprobe KR 261 von Kirchheim am Ries 


\section{$1 \quad$ Einleitung}

Erkenntnisse über historische Bevölkerungen zu gewinnen, ist ein Prozeß der Nutzbarmachung überdauerter Informationsquellen. Das Wechselspiel zwischen Lebensbedingungen und Lebensweise von Menschen in vergangenen Zeiten kann nicht direkt erfahren werden. Ein diesbezüglicher Erkenntnisprozeß bleibt grundsätzlich darauf beschränkt, erhaltene Information erfahrbar zu machen und aus ihr die historische Wirklichkeit zu rekonstruieren. Basale Voraussetzung für eine hierauf ausgerichtete Auswertung und Interpretation jeglicher Art historischer Informationsquellen ist die Kenntnis des Überlieferungsprozesses. Alle Aspekte der Historie der Information, ihre Genese, ihre Konservierung und nicht zuletzt auch die Möglichkeiten ihrer Diagenese (im weiteren Sinne) sind zu beachten. Erst durch die Einbeziehung aller den Informationsgehalt beeinflußenden Faktoren können die aus einer Informationsquelle gewonnenen Erkenntnisse und Folgerungen objektiviert werden.

Für die Historische Anthropologie ist bodengelagertes Skelettmaterial die entscheidende und oftmals einzige überdauerte Informationsquelle. Die Rekonstruktion von Lebensbedingungen historischer Bevölkerungen erfolgt unter Auswertung des Informationsgehaltes der überlieferten Knochen. Diese beinhalten zunächst Art und Weise der Bestattung, vor allem aber morphologische (makro- und mikroskopische), molekulargenetische und chemische Merkmale. Durch die Integration der Individualbefunde (Alter, Geschlecht, etwaige Pathologie, Verwandtschaft, Ernährungssituation usw.) in Gruppenbefunde wird der Versuch unternommen, die bestatteten Bevölkerungen zu charakterisieren und für deren Lebensweisen und Lebensbedingungen sozio-ökologische Szenarien zu rekonstruieren (vgl. Herrmann 2001).

Innerhalb dieses Forschungsrahmens befaßt sich die vorliegende Arbeit mit dem Skelettmaterial als chemischer Informationsquelle. Es wird der Informationsgehalt des Knochenminerals in Form der in ihm enthaltenen Spurenelemente ausgewertet. Zusammengefaßt erfolgt die Interpretation dieser Elementkonzentrationen auf der Grundlage bekannter, spezifischer Wechselwirkungen der Knochensubstanz als Bestandteil des menschlichen Organismus mit dessen Umwelt.

Der einleitenden Prämisse folgend beinhaltet das zu untersuchende Gefüge KnochenUmwelt zwei Wirkungskreise. Einerseits jenen der Informationsspeicherung zu Lebzeiten 
der Individuen, dem eigentlichen Gegenstand dieser Forschungsrichtung, und andererseits die Prozesse einer möglichen Informationsdiagenese nach dem Tode.

Wie im folgenden Kapitel gezeigt wird, sind für Zusammenhänge der Knochenchemie zu Lebzeiten kontinuierliche Erkenntniserweiterungen zu verzeichnen, aus welchen konsequent Limitierungen der Aussagemöglichkeiten abgeleitet wurden.

Aus dem darauffolgenden Kapitel bezüglich möglicher Informationsveränderungen respektive Konzentrationsveränderungen des Knochenminerals nach dem Tode wird eine unzureichend kritische Forschungsstrategie deutlich werden. Bezüglich der Berücksichtigung diagenetischer Veränderungen des Knochens bei der Interpretation von Spurenelementkonzentrationen sollte man sich Poppers auf wissenschaftliches Erkenntnisstreben allgemein bezogener Warnung entsinnen: "Die Suche nach objektiver Wahrheit ist bedroht von unserer Hoffnung, sie bereits gefunden zu haben" (Popper 1993, S. VIII).

Genau jene "Hoffnung" hat Fehlinterpretationen der chemischen Information bodengelagerten Skelettmaterials zur Folge. Die Aufklärung solcher Irrtümer, verbunden mit der Frage nach der Tauglichkeit der bisherigen Lösungsstrategien einschließlich der Überprüfbarkeit dieser Lösungsansätze, sind Gegenstand der folgenden Abhandlung.

\section{$1.1 \quad$ Ernährungsrekonstruktion durch Interpretation von Spurenelementgehalten in bodengelagertem Skelettmaterial}

Hauptziel der Auswertung von Spurenelementgehalten in bodengelagertem Skelettmaterial ist es, aus den Knochengehalten auf das Elementangebot in der zugeführten Nahrung zu schließen und damit die Ernährungssituation der untersuchten historischen Bevölkerungen zu rekonstruieren.

Das Prinzip dieser Ernährungsrekonstruktion gründet auf den Präferenzen, die Organismen im Zusammenhang mit dem Stoffaustausch bei Nahrungsaufnahme und Verstoffwechselung haben. Bestimmte Stoffe bzw. Elemente haben physiologische Relevanz und werden daher bevorzugt aus der Nahrung in den Organismus inkorporiert. Andere werden physiologisch ignoriert oder es wird bei schädlichen Folgen für den Organismus die Aufnahme gezielt unterbunden.

Für das Element Calcium gibt es für alle Organismen eine gleichgerichtete Präferenz zur Anreicherung. Hieraus resultiert innerhalb eines Nahrungsnetzes bei Übergängen von niederen zu höheren Trophiestufen ein Anreicherungs- bzw. Aufreinigungseffekt für dieses 
Element. Im Zuge dieser Biopurifikation des Calciums (vgl. Elias et al. 1982) wird gleichzeitig gegen die physiologisch unbedeutenden Elemente Barium und Strontium diskriminiert. Dies führt zu deren Abreicherung innerhalb der Nahrungskette oder präziser, der "Kette der Trophiestufen".

Diese Abnahme der Konzentrationen von Barium und Strontium in Relation zu Calcium ist der basale Zusammenhang, auf welchen Spurenelementuntersuchungen von Knochenmaterial aufsetzen.

Grundlage hierfür ist die knochenspezifische Mineralmatrix. Die anorganische Komponente des Knochens besteht im Wesentlichen aus verschiedenen Calcium-PhosphorVerbindungen, wobei die Hauptkomponente Hydroxylapatit $\left[\mathrm{Ca}_{10}\left(\mathrm{PO}_{4}\right)_{6}(\mathrm{OH})_{2}\right]$ ist (Driessens 1980; vgl. auch Pate \& Brown 1985; Posner 1978). Entscheidendes Charakteristikum dieser Verbindungen ist ihre kristalline Struktur. In diesen Kristallen besteht prinzipiell die Möglichkeit von Substitutionen der Calciumatome durch Strontium und Barium.

Der Mensch ist als Nahrungskonsument in das Nahrungsnetz eingebunden. Seine physiologischen Prozesse folgen bei der Nahrungsresorption dem Diskriminierungsprinzip gegen Barium und Strontium zu Gunsten von Calcium. Da die Diskriminierung ein relativer Prozeß ist, verbleibt von beiden Elementen für den Knochenstoffwechsel ein "Restangebot" in Relation zu Calcium, das von der Nahrungskomposition abhängt. Folglich werden Barium und Strontium bei Auf- bzw. Umbauprozessen der Knochensubstanz in das Kristallgitter der Knochenmatrix integriert. Die hierbei noch wirksame Diskriminierung gegen beide Elemente auf der kristallchemischen Ebene (vgl. Stark 1968) ist im Vergleich zur ernährungsphysiologischen von untergeordneter Bedeutung. In Abhängigkeit von der ursprünglichen Relation in der Nahrung ergeben sich im Knochenmineral bestimmte Konzentrationsverhältnisse zwischen den beiden Elementen Barium und Strontium in Bezug auf Calcium. Hierbei ist zu beachten, daß die Knochenumsatzrate gering ist und folglich die im Skelett gemessenen Elementgehalte die durchschnittliche Mineralzusammensetzung einer längerfristigen Ernährungsperiode widerspiegeln.

Für die Ernährungsrekonstruktion werden diese beiden Konzentrationsverhältnisse bzw. -quotienten als $\mathrm{Ba} / \mathrm{Ca}$ - und $\mathrm{Sr} / \mathrm{Ca}-$ Verhältnis der Knochensubstanz ausgewertet.

Die Interpretation der Konzentrationsquotienten erfolgt unter Berücksichtigung der generellen Abreicherung von Barium und Strontium von Trophiestufe zu Trophiestufe, denn hieraus resultiert ein Gradient von höheren $\mathrm{Ba} / \mathrm{Ca}$ - und $\mathrm{Sr} / \mathrm{Ca}-\mathrm{Verhältnissen} \mathrm{in} \mathrm{pflanzlichen}$ 
Nahrungskomponenten zu niedrigeren Werten in tierischen Produkten (vgl. Runia 1988). Hiermit ist auf der Basis empirisch quantifizierter Konzentrationsrelationen zwischen Nahrung und Knochenmineral eine Abschätzung der Ernährungsgrundlage möglich. Für Sr/Ca ist diese Relation als observed ratio etabliert (0,25 nach Comar et al. 1957, bzw. 0,2 nach Daten von Elias et al. 1982). Nach den Daten der letztgenannten Autoren kann eine solche Relation auch für $\mathrm{Ba} / \mathrm{Ca}$ mit einem Wert von 0,1 abgeleitet werden.

Zwischenzeitlich wurde das für die Ernährungsrekonstruktion auszuwertende Elementspektrum erweitert. Im Mittelpunkt stand hierbei Zink, außerdem wurden auch Eisen und Magnesium in die Modelle integriert und in verschiedenen Kontexten interpretiert (vgl. z.B. Schutkowski 1995; Buikstra et al. 1989; Francalacci \& Borgognini Tarli 1988 Blakely \& Beck 1981). Diese Elemente sind jedoch physiologisch erforderlich und unterliegen daher teilweise der körpereigenen Homöostase. Die Knochenkonzentrationen variieren damit nicht zwingend nahrungsabhängig. Eine strukturelle Einbindung in das Knochenmineral wie für Barium und Strontium ist ebenfalls nicht gesichert, wodurch die Umsatzrate nicht abschätzbar ist. Ein weiteres Problem ist die Tatsache, daß diese Elemente nicht hinreichend Trophiestufen oder spezielle Nahrungskomponenten charakterisieren (zusammenfassend in Ezzo 1994). Infolge der zunehmend kritischen Bewertung der Einbindung dieser Elemente in die Ernährungsrekonstruktion werden schließlich wieder nur Barium und Strontium in Relation zum Calciumgehalt des Knochens als verlässliche nahrungsanzeigende Spurenelemente präferiert (vgl. Sandford \& Weaver 2000).

Für die Auswertung der Konzentrationen beider Elemente als Repräsentanz für pflanzliche und tierische Nahrung in unterschiedlichen Anteilen erfolgte inzwischen eine weitere Einschränkung. Ursache hierfür sind die divergierenden Absolutmineralgehalte in verschiedenen Nahrungskomponenten. Bei Mehrkomponentenernährung ist eine lineare Umsetzung der Knochenkonzentrationen in quantifizierte Proportionen der einzelnen Komponenten nicht möglich. Mineralreiche Nahrungsbestandteile dominieren die Konzentrationsverhältnisse in der Nahrung. Mögliche größere Nahrungsanteile mineralarmer Komponenten können dadurch maskiert werden. Die $\mathrm{Ba} / \mathrm{Ca}$ - und $\mathrm{Sr} / \mathrm{Ca}-W e r t e$ des Knochenminerals spiegeln hiernach nur das Verhältnis der mineralreichen Hauptkomponenten der Nahrung wider (Burton \& Wright 1995). Experimentelle Vergleiche zwischen definierter Nahrungszufuhr und daraus resultierenden Knochenkonzentrationen deuten auf weitere Disproportionen zwischen Nahrungs- und Knochenkonzentrationen (Lambert \& Weydert-Homeyer 1993). 
Unabhängig von einer direkten Zuordnung von Konzentrationsverhältnissen und Nahrungszusammensetzung bleiben Konzentrationsunterschiede zwischen Individuen, Gruppen und Bevölkerungen auf der Basis der Ernährungsrekonstruktion interpretierbar. Innerhalb historischer Bevölkerungen sind hiernach entsprechende Auswertungen auf den verschiedenen sozio-ökologischen Interpretationsebenen prinzipiell möglich. So wurden auf Individualniveau ontogenetische Trends insbesondere im Kontext des Reproduktionsverhaltens erfaßt (z.B. Sillen \& Smith 1984, Blakely 1989). Auf Populationsniveau bildete die Charakterisierung sozialdifferenzierter Ernährungsqualitäten einen Schwerpunkt (z.B. Schoeninger 1979, Schutkowski 1995, Schutkowski et al. 1999). Populationsvergleichend erschließen sich diachrone Subsistenztrends (z.B. Grupe \& Schutkowski 1989, Katzenberg 1984) sowie in geographischer Habitatsvariabilität begründete Subsistenzdivergenzen (z.B. Schutkowski \& Herrmann 1996). Auf der Grundlage einer habitatabhängig variablen Verfügbarkeit von Barium und Strontium konnte durch kombinierte Auswertung beider Elementgehalte insbesondere auch zwischen terrestrischen und marinen Nahrungsgrundlagen unterschieden werden (Burton \& Price 1990b). Mit dem gleichen Prinzip war die Charakterisierung von Nahrungsressourcen aus Wüstengebieten möglich (Burton \& Price 1990a).

Es wird deutlich, daß die Etablierung und Anwendung des Konzeptes der Ernährungsrekonstruktion mit einen Wechsel von Modellerweiterungen und -einschränkungen verbunden ist. Die Probleme sind aber modellimmanent. Sie resultieren einzig aus der Berücksichtigung ernährungs- und stoffwechselphysiologischer Gesetzmäßigkeiten zu Lebezeiten der Individuen. Hiermit wird nur der Aspekt der Speicherung der chemischen Information im Knochenmineral erfaßt. Probleme der Informationskonservierung unter der Liegezeit des Skelettmaterials im Boden und die damit einhergehende Möglichkeit der Diagenese der chemischen Information bedürfen einer eigenen Erörterung. Sich daraus ergebende Einschränkungen der Interpretationsansätze werden in Kombination mit den Ergebnissen der experimentellen und statistischen Überprüfungen der vorliegenden Arbeit zu diskutieren sein.

\subsection{Die Kontroverse zur Diagenese der Knochensubstanz - ein (v-)erkanntes Problem?}

Die Konzentrationsverhältnisse in bodengelagertem Skelettmaterial zur Rekonstruktion sozio-ökologischer Szenarien historischer Bevölkerungen auszuwerten erfolgt unter der Vorannahme, daß die im Knochen analysierten Elementgehalte die Konzentrationsverhält- 
nisse der Individuen zu deren Lebzeiten (in vivo) widerspiegeln. Die Konzentrationsinformationen werden als authentisch vorausgesetzt.

Während der Bodenlagerung der Knochensubstanz können jedoch Konzentrationsänderungen nicht ausgeschlossen werden. Eine Diagenese (hier und im Folgenden i.e.S.) des Knochens muß in Betracht gezogen werden. Als solche sind für die weiteren Betrachtungen alle bodenchemisch bedingten und mikrobiellen Veränderungen der Knochensubstanz zu definieren. Ebenfalls als diagenetische Veränderung einzubeziehen sind Substanz- respektive Elementeinträge infolge postmortaler Auflagerungen auf die Knochenoberfläche bzw. Einträge in Hohlräume der Knochensubstanz, die keine chemische Wechselwirkung einschließen.

Bezüglich der Existenz dieses Problems besteht wissenschaftlicher Konsens. Seine Relevanz sowie Lösungsstrategien für die Interpretation von Knochenkonzentrationen werden seit langem kontrovers diskutiert.

Von jenen Elementen, die mit den genannten ernährungs- bzw. knochenphysiologischen Begründungen aus der Ernährungsrekonstruktion zu eliminieren waren, finden sich u.a. Magnesium und Eisen unter den ersten diagenetisch kritischen Kandidaten. Die hohe Mobilität dieser Elemente und/oder allgemein erhöhte Konzentrationen im Boden schließen sie im Rahmen der Diagenesediskussion von einer weiteren Verwendung zur Ernährungsrekonstruktion aus (vgl. Ezzo 1994). Es ist erstaunlich, daß Magnesium ursprünglich als relativ unempfindlich gegenüber diagenetischen Veränderungen bewertet wurde (zu dieser Diskussion vgl. Pate 1994, S. 183ff). Da aus den in 1.1 (S. 4) genannten Gründen das weitere Interesse an diesen Elementen nachgelassen hat, ist der Diskussionsbedarf für diese (und die anderen im Kontext der Ernährungsrekonstruktion nicht mehr relevanten) Elemente gering.

Dies verhält sich bezüglich Strontium grundsätzlich anders. Das für die Ernährungsrekonstruktion wichtigste Spurenelement (nicht zuletzt, weil nur Strontium und Barium noch als auswertbare Elemente empfohlen werden, s.o.) zeigt das Problem der Diagenesediskussion am deutlichsten.

In der Initialpublikation zur Verwendung von Elementkonzentrationen in Skelettmaterial für ernährungsökologische Fragestellungen wird für Strontium eine hinreichende Unempfindlichkeit gegenüber liegezeitbedingten Konzentrationsveränderungen postuliert (Toots \& Voorhies 1965). Diese Einschätzung wurde wiederholt bekräftigt (z.B. Parker \& Toots 
1970; Parker \& Toots 1980; Lambert et al. 1979; Lambert et al. 1982; Burton \& Price 1990b). Als "Sicherheitsoption" wird die Verwendung von Kompaktaproben der Langknochen vorgeschlagen, da diese diagenetischen Einflüssen gegenüber besonders stabil seien (Pate \& Brown 1985).

Eine zunehmend kritischere Bewertung von Knochenkonzentrationen wurde zunächst auf diagenesebegünstigende Bodenbedingungen (geringer $\mathrm{pH}$-Wert mit entsprechend erhöhter Ionenmobilität, vgl. Pate \& Hutton 1988) oder lange Zeiträume beschränkt (Sillen 1981). Unter Berücksichtigung boden- sowie knochenmineralchemischer Wechselwirkungen (die Komplexität derselben ist bereits in Pate \& Hutton 1988 erwähnt) wird inzwischen generell die Möglichkeit von relevanten diagenetischen Konzentrationsveränderungen (auch für Strontium) eingeräumt (zusammenfassend in Price et al. 1992; Ezzo 1994; Pate 1994).

Diesem Prozeß der Einsicht folgte die Einführung und Überprüfung von Probenaufreinigungsverfahren. Beschränkten sich die Methoden zunächst auf eine mechanische Oberflächenbehandlung der $\mathrm{zu}$ analysierenden Knochenproben (Lambert et al. 1989), konzentrierte sich die Aufreinigung sehr schnell auf eine chemische Variante (Sillen 1986). Anhand von Modellen zur Knochendiagenese, einschließlich experimenteller Überprüfungen, etablierte sich "ein Prinzip" der Probenaufreinigung, welches insbesondere für Strontium getestet wurde (Sillen 1986; Sillen \& LeGeros 1991). Dieses Prinzip sowie verschiedene inzwischen genutzte Modifikationen basieren auf den differentiellen Löslichkeiten von nativem bzw. diagenem Knochenmineral z.B. infolge variablem Anteils von Carbonat (vgl. Sillen 1989) oder leichter löslicherer Rekristallisationsprodukte im Zuge des Hydroxylapatitab- bzw. -umbaus (vgl. Herrmann \& Newesely 1982). Natives (in vivo gebildetes) Knochenmineral ist hiernach in Lösungen mit geringem $\mathrm{pH}$-Wert (durch Säurezusatz) wegen seiner geringeren Löslichkeit stabiler als diagenetisch verändertes Knochenmineral. Durch die erhöhte Löslichkeit wird letzteres überproportional aus der Probe entfernt und bei einem hinreichenden Lösungsprozeß verbleibt nur noch natives Knochenmineral in der Probe (Überblick zu den Methodenvarianten z.B. in Price et al. 1992).

Abgesehen von der Diskussion über verschiedene Varianten solcher Aufreinigungen (Sillen \& Sealy 1995 vs. Nelson et al. 1986) gibt es grundsätzlichen Widerspruch gegen diese Methoden. Auf der Basis eines erweiterten Verständnisses der Diagenese als eines eher multi- denn unidirektionalen Prozesses wurde die hinreichende Effizienz, sowie das Löslichkeitsprinzip als solches wiederholt in Frage gestellt (eine Auswahl: Hanson \& Buikstra 1987; Tuross et al. 1989; Pate et al. 1989; Radosevich 1993; Quattropani et al. 
1999; Budd et al. 2000; Nielsen-Marsh \& Hedges 2000b). Man beachte die lange Zeitspanne von 1987 bis einschließlich heute, in welcher die Zweifel wiederholt werden. Inwieweit in Anbetracht dieser Kritik die prinzipielle Beibehaltung dieser "Lösung des Diageneseproblems" ein Folge mangelnder Alternativen ist oder hierfür jene einleitend erwähnte "Hoffnung auf eine bereits gefundene Wahrheit" (vgl. S. 2) verantwortlich ist, soll offen bleiben. Tatsache ist, daß Spurenelementanalysen an bodengelagertem Skelettmaterial unter Verwendung säureinduzierter Aufreinigung der Knochenproben durchgeführt wurden und werden (vgl. zusammenfassend in Sandford \& Weaver 2000).

Die weiteren Untersuchungen bezüglich der Diagenese beschränken sich auf den Versuch, durch Kontrollparameter den Grad der diagenetischen Veränderung bzw. den des Aufreinigungserfolges $\mathrm{zu}$ prüfen. Die histologische Erhaltung der Knochenbinnenstruktur gilt hierbei als ein erstes Kriterium (z.B. Hanson \& Buikstra 1987; Schoeninger et al. 1989; Hedges et al. 1995). Erweitert umfassen solche Parameter Kontrollen der knochenmineralspezifischen Stöchiometrie von Calcium und Phosphor (als Ca/P-Verhältnis, vgl. z.B. White \& Hannus 1983 Hancock et al. 1989) sowie der Kristallinität der Mineralmatrix (z.B. Lee et al. 1995 Wright \& Schwarcz 1996; Quattropani et al. 1999; Nielsen-Marsh \& Hedges 2000a). Über Konzentrationsprofile bzw. -gradienten innerhalb der Knochenproben werden diagenetische Konzentrationsänderungen anhand ihrer Diffusionskomponente charakterisiert (z.B. Williams et al. 1997; Millard \& Hedges 1995; Reiche et al. 1999). Durch die kombinierte Auswertung der Konzentrationen möglichst vieler Elemente erfolgt der Versuch, korrelierende Elementveränderungen als Diageneseindikatoren zu spezifizieren (sog. Multielementstudien, z.B. Buikstra et al. 1989; Price 1989). Schließlich wurde auch bei einer Beschränkung auf die inzwischen allein präferierten ernährungsrelevanten Elemente Barium und Strontium eine aus der Ernährungsrekonstruktion abgeleitete Korrelation beider Elemente innerhalb einer Bevölkerung als Kontrollfunktion vorgeschlagen (Burton \& Price 1990a; Burton et al. 1999).

Damit sind Kriterien für die Charakterisierung diagenetischer Veränderungen am Knochenmineral hinreichend vorhanden. Abgesehen davon, daß es hinsichtlich ihrer allgemeinen Zuverlässigkeit selbst bei kombinierter Anwendung Zweifel gibt (vgl. Sillen 1989, Radosevich 1993; Hedges et al. 1995), bleibt ein entscheidendes Problem: Alle Parameter ermöglichen keine hinreichende Quantifizierung der diagenetischen Konzentrationsänderungen für die ernährungsrelevanten Elementkonzentrationen von Barium und Strontium, sondern allenfalls eine Abschätzung des allgemeinen Grades der Veränderung. 
Hiernach kann einer Forderung, primär sicherzustellen, nur in vivo generierte Elementsignaturen für die Ernährungsrekonstruktion einschließlich ihrer Folgeinterpretationen zu verwenden, nicht entsprochen werden. Es muß festgestellt werden, daß für die Auswertung von Elementgehalten bodengelagerten Skelettmaterials die aus diagenetischen Konzentrationsänderungen resultierenden Limitierungen nur in einem begrenzten Umfang definiert sind. Die Folge ist eine wissenschaftlich wenig zufriedenstellende Praxis mit dem Konsens, Elementgehalte des Knochenminerals human- bzw. sozio-ökologisch zu interpretieren, und mit Hinweis auf nicht auszuschließende diagenetische Interferenzen die Aussagesicherheit einzuschränken (vgl. Radosevich 1993).

Selbst diese Vorgehensweise einer unter Vorbehalt durchgeführten Interpretation setzt voraus, daß die Kontrollkriterien eine zuverlässige Einschätzung der diagenetischen Veränderungen erlauben. Eine Minimalforderung an ein Kontrollkriterium wäre eine hinreichend systematische Abgrenzung auswert- bzw. interpretierbarer Knochenkonzentrationen von diagenetisch maskierten Elementgehalten.

Diese Forschungssituation wurde in der vorliegenden Arbeit zum Anlaß genommen, eine kritische Evaluation für eine Auswahl frühere Auswertungen der Spurenelementdaten bodengelagerten Skelettmaterials dreier Gräberfelder durchzuführen. Von den Ergebnissen dieser Neuauswertungen wurden experimentelle Überprüfungen zum Problem der Knochenprobenaufreinigung abgeleitet. Ebenfalls resultierende Fragestellungen zur Tauglichkeit von diagenesecharakterisierenden Kontrollkriterien wurden in diese Experimente integriert.

Im ersten Untersuchungsteil wurden vorhandene Spurenelementdaten von drei Skelettpopulationen mit dem Ziel neu ausgewertet, Konzentrationsveränderungen in Abhängigkeit von diagenetischen Veränderungen der Einzelproben nachzuweisen. Ausgangspunkt bildete hierbei die Überlegung, daß im Falle des Vorhandenseins einer diagenetischen Konzentrationsbeeinflussung innerhalb einer Skelettpopulation systematische Unterschiede zwischen Knochenproben unterschiedlicher Erhaltung statistisch nachweisbar sein müßten. Über die histologische Erhaltung der Knochenproben als Maß für diagenetische Beeinflussung wurden innerhalb einer Population entsprechende Probengruppen definiert und diese Gruppen hinsichtlich ihrer $\mathrm{Ba} / \mathrm{Ca}$ - und $\mathrm{Sr} / \mathrm{Ca}-\mathrm{Werte}$ verglichen. Um ein mögliches diagenetisches Konzentrationsmuster von einem populationsinternen in vivo generierten abzugrenzen, wurde das diagenetische Gruppenkonstrukt dem früheren Interpretationsansatz einer ernährungsabhängigen Gruppenstruktur im soziokulturellen Kontext gegenüberge- 
stellt. Anschließend wurden für zwei weitere Skelettkollektive die Spurenelementdaten hinsichtlich diagenetischer Abhängigkeiten geprüft. Damit war die Erschließung einer Systematik diagenetischer Konzentrationsveränderungen mit statistischer Absicherung auf einer breiten Datenbasis möglich.

Die ursprünglichen ernährungsabhängigen Interpretationen der populationsinternen Konzentrationsmuster erfolgten im Rahmen des dargelegten wissenschaftlichen Konsenses bezüglich Spurenelementdaten von bodengelagertem Knochenmaterial. Die Konzentrationsdaten resultieren aus Analysen von Knochenproben, die nach dem wissenschaftlich akzeptierten Prinzip einer säureinduzierten Aufreinigung vorbereitet wurden. Nach der anschließenden Prüfung hinsichtlich eines ebenfalls akzeptierten Kontrollkriteriums für natives (in vivo bedingtes) Knochenmineral ( $\mathrm{Ca} / \mathrm{P}-$ Verhältnis) waren die Proben für eine Auswertung als hinreichend befunden worden. Daher ergab sich aus der Aufdeckung eines diagenetischen Konzentrationsmusters innerhalb der überprüften Bevölkerungen als logische Konsequenz eine experimentelle Kontrolle von Effizienz und prinzipieller Tauglichkeit der Aufreinigungsmethode sowie des diagenesecharakterisierenden Kontrollkriteriums. Ein Versuch zur Überprüfung der Aufreinigungsmethodik wurde deshalb im zweiten Untersuchungsteil integriert. Hierfür wurde eine Stichprobe einer zusätzlichen historischen Bevölkerung verwendet.

Die Überprüfung des Kontrollkriteriums für den Erhaltungszustand des Knochenminerals (Ca/P-Verhältnis) wurde um weitere diagenesecharakterisierende Kriterien ergänzt. Als eigenständiger Untersuchungsaspekt erfolgten entsprechend Auswertungen für die Daten der Knochenproben aus dem Aufreinigungsversuch sowie die der primär überprüften Untersuchungen.

Eine differenzierte Charakterisierung diagenetischer Veränderungen des Knochenminerals insbesondere im Zusammenhang mit den überprüften diagenesecharakterisierenden Kontrollkriterien erfolgte im vierten Untersuchungsteil durch mikroanalytische Untersuchungen einer bodengelagerten Knochenprobe.

Der letzte Teil ist losgelöst von der Problematik der Knochendiagenese. Die Analysen zur Konzentrationsvariabilität innerhalb eines Femurschaftes erfolgten ursprünglich im Kontext der Aufreinigungsüberprüfung als Kontrollexperiment. Die Ergebnisse werden als eigenständiges Problem für die Interpretation von Spurenelementgehalten im Knochenmaterial bewertet und daher entsprechend vorgestellt. 


\section{$2 \quad$ Konzeption der Untersuchungen}

Im Folgenden werden die verschiedenen Untersuchungsaspekte einzeln erläutert. Es werden die Auswertungsansätze und die Versuchsplanungen dargelegt. Die Strukturierung der Gesamtuntersuchung resultiert aus aufeinander aufbauenden Teilversuchen, d.h. die Fragestellungen verschiedener Teilversuche ergaben sich aus jeweils vorangegangenen Auswertungen. Die folgende ausführliche Darstellung dieses Untersuchungsdesigns dient einem verbesserten Verständnis der Vorgehensweise und wird deshalb dem Kapitel zu Material und Methoden vorangestellt. Im Rahmen der Erläuterungen ist es jedoch unvermeidlich, teilweise Informationen zum untersuchten Material bzw. auch methodische Aspekte zu integrieren. Dies bezieht sich insbesondere auf die als Ausgangspunkt für alle Fragestellungsaspekte dienende histologische Bewertung der Knochenbinnenstruktur. Vom Einzelversuch unabhängige Informationen zum Material sowie detaillierte Angaben zur Probenvorbereitung, Analytik und Datenauswertung werden jedoch erst im anschließenden Kapitel 3 aufgeführt.

Die dargestellte Versuchskonzeption entspricht einer chronologischen Abfolge von Fragestellungen. Dargestellt wird ein Prozeß beginnend mit der "Herleitung" des Problems der diagenetischen Beeinflussung von Knochenkonzentrationen (2.1). Es folgt die Überprüfung der Probenaufreinigungsmethode, die als bisherige Strategie zur Lösung des Diageneseproblems vorgeschlagen und angewendet wird (2.2). Im Zusammenhang mit der Tauglichkeit der Probenaufreinigung ergibt sich eine kritische Bewertung sowie Erweiterung von diageneseanzeigenden Kontrollkriterien (2.3) und der Versuch diagenetische Veränderungen mikroanalytisch zu charakterisieren (2.4). Der abschließende Untersuchungsaspekt zur intraossären Variabilität von Elementkonzentrationen (2.5) wird unabhängig von den anderen Teiluntersuchungen dargestellt.

\subsection{Die Möglichkeit diagenetischer Konzentrationsveränderungen: Überprüfungen zur populationsinternen Variabilität von $\mathrm{Ba} / \mathrm{Ca}$ - und $\mathrm{Sr} / \mathrm{Ca}$-Werten}

Die Überprüfungen auf mögliche diagenetische Veränderungen der Spurenelementkonzentrationen erfolgten im Hinblick auf die Konsequenzen für die Ernährungsrekonstruktion beschränkt auf die Elemente Barium und Strontium (als Ba/Ca- und $\mathrm{Sr} / \mathrm{Ca}-\mathrm{Quotienten}$ ), da nach der in 1.1 (S. 4) erwähnten Empfehlung diese Elemente im Mittelpunkt weiterer Forschungen stehen werden. 
Ein diagenetischer Einfluß auf Spurenelementkonzentrationen kann nur erfaßt werden, wenn er innerhalb einer Population zu systematischen Veränderungen von Elementkonzentrationen bzw. Veränderungen der genannten Konzentrationsquotienten führt. Die populationsinterne Konzentrationsvariabilität sollte Abhängigkeiten von diagenetischen Veränderungen zeigen.

Für die vorliegende Untersuchung wurde als diagenetischer Marker die histologische Erhaltung der Knochensubstanz gewählt. Der lichtmikroskopisch bewertete Erhaltungszustand der Knochenbinnenstruktur wird als ein von den gemessenen Spurenelementkonzentrationen unabhängiges, externes Bezugsystem verwendet. Hiermit werden innerhalb der Populationen Gruppen definiert, die anschließend hinsichtlich möglicher Unterschiede ihrer Elementkonzentrationen überprüft werden. Zu beachten ist hierbei, daß die histologische Gruppeneinteilung prinzipiell unabhängig vom makroskopischen Erscheinungsbild des Knochens ist. Es ist unerheblich, ob die Knochenproben von Knochenfragmenten oder aus einer intakten Gesamtstruktur entnommen wurden.

Die Definition dieser Histologiegruppen bildet die Grundlage für die verschiedenen Teilversuche zur Charakterisierung diagenetischer Veränderungen. Die Vorgehensweise zur Einordnung der Knochenproben in Gruppen unterschiedlicher histologischer Erhaltung wird daher den weiteren Erörterungen vorangestellt.

\section{Histologiegruppen als diagenetische Gruppen}

Die Bewertung histologischer Präparate zur Charakterisierung des Erhaltungszustandes und damit zur Abschätzung diagenetischer Veränderungen der Knochensubstanz wird in der Literatur wiederholt genannt (Hanson \& Buikstra 1987; Schoeninger et al. 1989; Hedges et al. 1995; Pfeiffer \& Varney 2000). Hierbei ist zu beachten, daß in Abhängigkeit vom Kontext (z.B. Kollagengehalt vs. Kollagenerhaltung) in den beiden letztgenannten Publikation kritische Anmerkungen enthalten sind, aber als rein phänomenologische Charakterisierung der Erhaltung der Knochenbinnenstruktur bleibt diese Methode unbestritten.

Für diese Untersuchung erfolgte die Definition der Histologiegruppen auf der Grundlage der Kategorien von Hedges et al. (1995). Deren Einteilung nach dem geschätzten prozentualen Flächenanteil histologisch intakter Knochenbinnenstruktur im Kompaktaquerschnitt ist in sechs Kategorien untergliedert. Wie der Publikation zu entnehmen, sind die mittleren Kategorien meist nur mit wenigen Proben besetzt. Hinzu kommt die Problematik einer 
genauen Zuordnung von Proben in die sechs Einzelkategorien. Für eine praktikable Einteilung der Knochenproben und zur Sicherstellung einer statistisch relevanten Auswertung wurde daher entschieden, zunächst jeweils zwei dieser sechs Kategorien zu einer zusammenzufassen. Damit ergaben sich drei Kategorien, in welche die Knochenproben aller in dieser Arbeit untersuchten Skelettpopulationen eingeordnet wurden. Diese Kategorien bzw. Histologiegruppen wurden mit gut, mittel und schlecht bezeichnet. Aus dieser Zusammenfassung zu drei Kategorien resultieren folgende Vorgaben für eine Abschätzung des Flächenanteils histologisch intakter Knochenbinnenstruktur innerhalb eines Kompaktaquerschnittes:

- gut:

Anteil der intakten Struktur

größer $85 \%$

- mittel:

Anteil der intakten Struktur

zwischen $15 \%$ und $85 \%$

- schlecht:

Anteil der intakten Struktur

kleiner $15 \%$

Durch diese Einteilung wurde erreicht, daß mit gut und schlecht zwei klar abgegrenzte Histologiegruppen definiert sind. Die mittlere Gruppe ist durch den weiten Bereich der möglichen Einordnung per se sehr heterogen. Trotzdem ergaben sich für diese Gruppe nur wenige Proben. Das von Hedges et al. (1995) dokumentierte Phänomen wurde bestätigt. Bei einer histologischen Beurteilung von bodengelagerten Knochenproben wird ein entweder-oder-Muster deutlich. Die Knochenbinnenstruktur ist entweder großflächig unverändert oder nahezu vollständig zerstört. Infolge der heterogenen Zusammensetzung der Histologiegruppe mittlerer Erhaltung sowie ihre für alle untersuchten Populationen geringen Besetzung wurde diese Kategorie von den Auswertungen ausgeschlossen. Nicht zuletzt würde durch drei Kategorien die Interpretierbarkeit der statistischen Auswertungen eingeschränkt.

Unabhängig von diesem Probenausschluß werden in den Abbildungen 1 bis 3 auf den folgenden Seiten anhand von Beispielen für alle drei Histologiegruppen die lichtmikroskopisch erfaßten Charakteristika zur Differenzierung zwischen intakten und veränderten Arealen der Knochenbinnenstruktur erläutert (zur Herstellung der histologischen Präparate vgl. 3.2.1.2).

Jede Abbildung enthält eine Übersichtsaufnahme des Kompaktaquerschnittes sowie eine vergrößerte Detailaufnahme zur Hervorhebung spezifischer Veränderungen bzw. bei Abbildung 1 zur Darstellung der intakten Knochenbinnenstruktur. 
In Abbildung 1 ist die Histologie einer als gut erhalten eingeordneten Knochenprobe dargestellt. Osteonstrukturen einschließlich der konzentrischen Lamellenanordnung mit Osteozytenspalten um die Haversschen Kanäle sind vollständig erkennbar. Auffällige Veränderungen des Knochengefüges können nicht festgestellt werden.

Das Beispiel einer als mittel erhalten bestimmten Histologie in Abbildung 2 zeigt periostseitig einen schmalen sowie zum Markraum einen breiteren Bereich mit ebenfalls intakter Knochenstruktur. Dazwischen sind jedoch Strukturen sichtbar, die als diagenetisch veränderte Knochensubstanz angesprochen werden. Solche Strukturen stellen sich sowohl als helle blasig erscheinende Hohlräume wie auch sich dunkel abzeichnende gefüllte Hohlräume dar (beide Hohlraumstrukturen wahrscheinlich mikrobiellen Ursprungs, vgl. Grupe et al. 1993). Die Detailaufnahme (Abbildung 2b) verdeutlicht den Übergang von intakter zu veränderter Knochenstruktur. Bei dem Osteon am linken unteren Bildrand ist die konzentrische Lamellenstruktur um den Haversschen Kanal noch deutlich erkennbar und nur der äußere Bereich leicht gestört. Von dem rechts anschließenden Osteon ist nur noch der Haverssche Kanal sichtbar, die restliche knochenspezifische Struktur ist komplett umgebildet. Das gezeigte Beispiel ist wegen der beiden nebeneinander vorkommenden Strukturtypen als typisch mittel erhalten einzuordnen. Außerdem ist die beobachtete Anordnung als Schichtung von Periost bis zum Markraum (gut-schlecht-gut erhalten) für viele Proben dieser Kategorie charakteristisch.

Der Kompaktaquerschnitt der als schlecht kategorisierten Probe in Abbildung 3 unterscheidet sich von Abbildung 2 dadurch, daß als einzige intakte Struktur periostseitig nur noch ein sehr schmaler Streifen vorhanden ist. Der restliche Querschnitt ist bis auf die Öffnungen der Haversschen Kanäle durch eine vollständige Strukturauslöschung gekennzeichnet.

Es wird deutlich, daß eine Zuordnung zu den beiden Histologiegruppen gut und schlecht prinzipiell unkritisch ist. Für die Grenzfälle der problematischeren Entscheidung zwischen gutem und mittlerem sowie zwischen mittlerem und schlechtem Erhaltungszustand war eine eindeutige Konvention erforderlich. Im Zweifelsfall erfolgte daher grundsätzlich eine Zuordnung zur mindererhaltenen Kategorie.

Die histologischen Präparate der für diesen ersten Versuchsteil verwendeten drei Skelettkollektive (Weingarten, Kirchheim unter Teck und Wenigumstadt, vgl. 3.1.1) wurden in Zusammenhang mit früheren Untersuchungen bereits einmal bewertet. Es galt einen ein- 
heitlichen Bewertungsmaßstab für die Einordnung der Knochenproben in die drei definierten knochenhistologischen Erhaltungskategorien zu gewährleisten. Daher erfolgte für die vorliegende Untersuchung eine vollständige Neubewertung der Histologien aller drei Skelettserien unter konsequenter Einhaltung der erläuterten Kriterien. Aus der genannten Zuordnungskonvention resultierte eine gegenüber früheren Bewertungen teilweise systematisch veränderte Einordnung der Proben in die Histologiegruppen.

Im einzelnen erhöht sich die Anzahl der als schlecht erhalten bewerteten Proben, wohingegen die Anzahl der gut erhaltenen Proben abnimmt. Die Gruppe der Proben mit mittlerer Erhaltung wird durch die Zuordnung bisher als gut erhalten eingeteilter Proben zahlenmäßig vergrößert und wiederum durch die neue Zuordnung ihr bisher zugehöriger Proben in die Gruppe der schlecht erhaltenen verringert. Das Resultat der neuen Histologiebeurteilungen ist daher eine "Zuordnungsverschiebung" der Knochenproben zu tendenziell schlechteren Histologien ${ }^{1}$.

Zusätzlich zu den erläuterten systematischen Zuordnungsverschiebungen resultieren aus der Neubewertung der Histologien durch nichtidentische Beobachter unweigerlich subjektive Bewertungsabweichungen gegenüber den früheren Beurteilungen. Diese führen infolge der weit gefaßten und daher vereinfachten Bewertungskategorien (vgl. S. 12f) nur für wenige Einzelproben zu abweichenden Beurteilungen. Innerhalb der statistischen Auswertungen der Konzentrationsdaten sollten hieraus resultierende möglich Fehler nivelliert werden.

Selbstredend erfolgte die Neubewertung der Histologien für die bereits elementanalytisch untersuchten Skelettpopulationen unabhängig von den probenzugehörigen Konzentrationsdaten, d.h. ohne deren Kenntnis bzw. unter Ausschluß einer direkten Vergleichsmöglichkeit.

\footnotetext{
${ }^{1}$ Es ist an dieser Stelle auf folgenden Sachverhalt hinzuweisen:

Durch diese Vorgehensweise sind insbesondere Fehleinordnungen von Proben mit mittlerem Erhaltungszustand in die Gruppe der schlecht erhaltenen möglich. Hierdurch kann ein erhaltungsabhängiges Konzentrationsmuster innerhalb einer Skelettpopulation nur falschnegativ maskiert werden, denn: Falsch zugeordnete, eigentlich mittel erhaltene Proben würden eine vorhandene gruppenspezifische Konzentrationscharakteristik der schlecht erhaltenen Proben in Richtung besser erhaltene verfälschen.

Andererseits gilt: Gut erhaltene Proben, die infolge der Einordnungskonvention fälschlich in die Gruppe der mittel erhaltenen eingeordnet wurden, bedeuten zwar eine zahlenmäßige Verminderung der ersteren, aber keine Verfälschung ihrer Erhaltungscharakteristik. Die Gruppe der mittel erhaltenen ist für die Auswertung irrelevant.

Infolge der verwendeten Zuordnungsvorschrift sind daher artifizielle Konzentrationsgruppen unwahrscheinlich.
} 

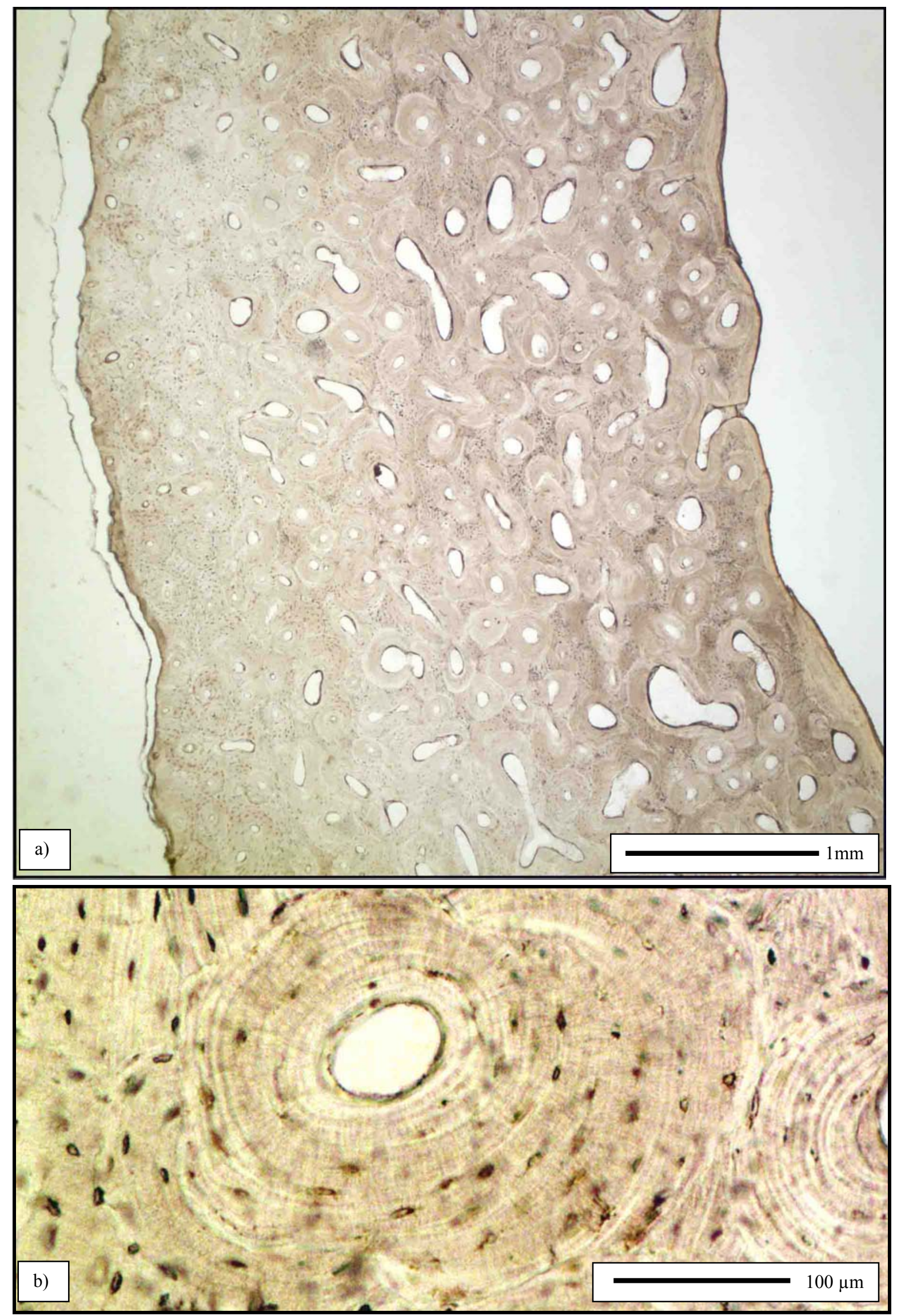

Abbildung 1: Lichtmikroskopische Aufnahmen eines Knochendünnschnittes von einer histologisch als gut kategorisierten Knochenprobe von Kirchheim am Ries (KR 106).

a) Übersicht, b) Detailausschnitt (vgl. Text) 

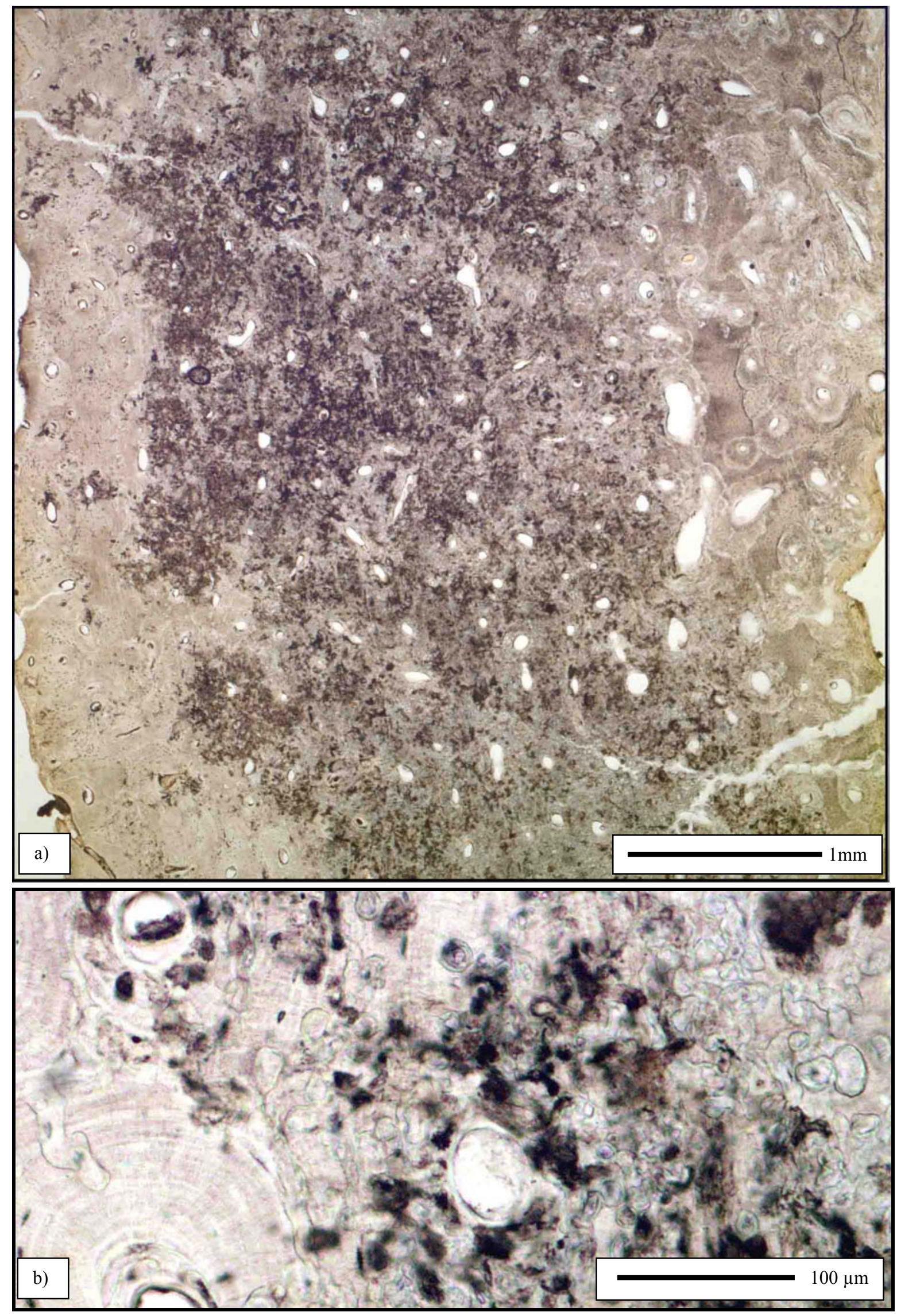

Abbildung 2: Lichtmikroskopische Aufnahmen eines Knochendünnschnittes von einer histologisch als mittel kategorisierten Knochenprobe von Kirchheim am Ries (KR 261).

a) Übersicht, b) Detailausschnitt (vgl. Text) 

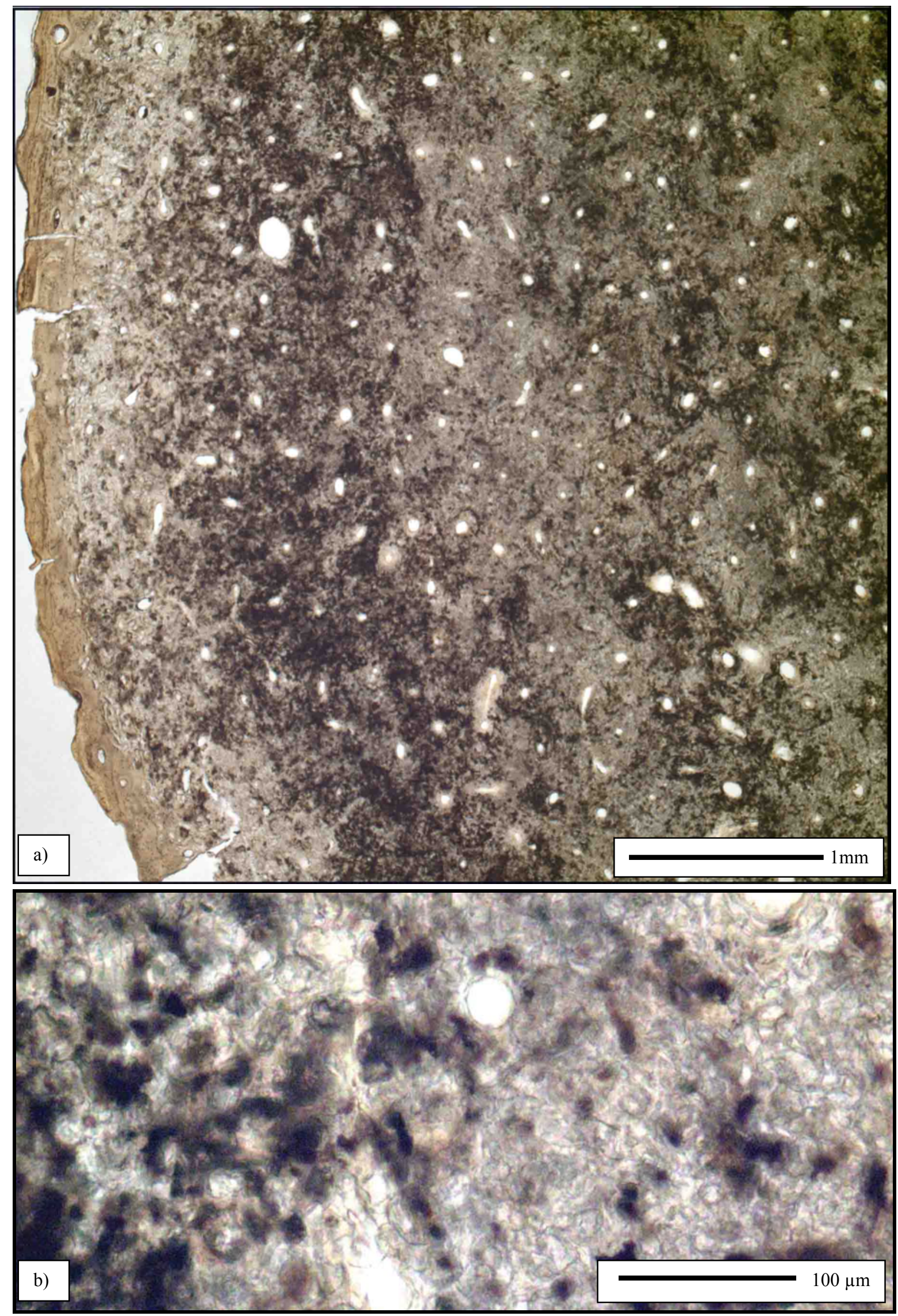

Abbildung 3: Lichtmikroskopische Aufnahmen eines Knochendünnschnittes von einer histologisch als schlecht kategorisierten Knochenprobe von Kirchheim am Ries (KR 206).

a) Übersicht, b) Detailausschnitt (vgl. Text) 
Mit diesem histologischen Einteilungssystem wurden die Knochenproben der drei historischen Bevölkerungen (Weingarten, Kirchheim unter Teck und Wenigumstadt) populationsintern primär in drei Gruppen ( gut/mittel/schlecht) eingeordnet. Von diesen drei wurden für die statistischen Auswertungen zwei Gruppen (gut/schlecht) verwendet (vgl. S. 13).

Die beiden histologisch definierten Gruppen (gut/schlecht) wurden als Entsprechungen für Knochenproben mit unterschiedlichen diagenetischen Veränderungen verstanden. Wenn diagenetische Veränderungen der Knochenbinnenstruktur systematische Änderungen der eigentlich ernährungsanzeigenden Elementrelationen von Barium und Strontium in Bezug auf Calcium verursachen, sollten sich populationsintern zwischen diesen beiden Probengruppen Unterschiede hinsichtlich ihrer $\mathrm{Ba} / \mathrm{Ca}$ - und $\mathrm{Sr} / \mathrm{Ca}-W e r t e$ ergeben. Dies galt es statistisch zu überprüfen.

Alle drei genannten historischen Bevölkerungen sind in früheren Forschungsvorhaben zur Ernährungsrekonstruktion innerhalb soziokultureller Interpretationsansätze bereits einmal ausgewertet worden (vgl. Schutkowski 1995; Schutkowski 1998; Schutkowski et al. 1999). Die archäologischen und anthropologischen Informationen zu diesen historischen Bevölkerungen in 3.1.1 sind jedoch für das Verständnis der hier erläuterten Fragestellung nicht erforderlich, sie werden an der genannten Stelle gemeinsam mit den Angaben zum Material für die anderen Untersuchungsteile formal aufgeführt.

\section{Populationsinterne Gegenüberstellung von diagenetischen und ernährungsbedingten Gruppen}

Auf die Interpretationen früherer Auswertungen der drei historischen Bevölkerungen wird in der vorliegenden Arbeit nur hinsichtlich einer der Populationen (Weingarten, vgl. 3.1.1, S. 39) eingegangen. Für Weingarten wurde die Hypothese einer möglichen diagenetischen Beeinflussung der $\mathrm{Ba} / \mathrm{Ca}$ - und $\mathrm{Sr} / \mathrm{Ca}-W e r t e$ den ursprünglichen Interpretationen ernährungsbedingter Unterschiede beider Variablen gegenübergestellt. Hierzu sind zusätzlich zur histologischen Gruppendefinition weitere aus den früheren Auswertungen ableitbare Gruppenstrukturen in die statistischen Auswertungen integriert worden.

Für das Verständnis dieser Vorgehensweise ist zunächst zu beachten, daß die statistische Auswertung der früheren Untersuchung zu Weingarten sich prinzipiell von der für die vorliegende Untersuchung unterscheidet. Wurden für die Überprüfung Histologiegruppen als externes Bezugssystem definiert, in welches die Knochenproben eingeordnet wurden, er- 
folgte ursprünglich eine ansatzfreie Clusteranalyse anhand der Spurenelementdaten. Es wurden also zunächst explorativ Gruppen ohne externen Bezug gebildet. In diese Clusteranalyse gingen zusätzlich zu den Konzentrationen von Barium und Strontium noch die von Zink und Kupfer ein (vgl. Schutkowski et al. 1999).

Unabhängig von der in 1.1 (S. 4) dargelegten allgemeinen Problematik der Verwendung von Zink innerhalb der Ernährungsrekonstruktion wird dieses Element sowie Kupfer bei der weiteren Betrachtung vernachlässigt, weil die mit der Clusteranalyse ermittelten Gruppen (Cluster) sich hauptsächlich über die Barium- und Strontiumgehalte der Knochenproben definierten. Bei den berechneten drei Gruppen ist eine deutliche Zweiteilung hinsichtlich der beiden Elementgehalte festzustellen (ein Cluster mit hohen und ein Cluster mit niedrigen Konzentrationen sowie eines mit einer analogen internen Differenzierung).

Die Cluster wurden in den ursprünglichen Auswertungen als Ernährungsgruppen interpretiert. Zwischen diesen konzentrationsdefinierten Ernährungsgruppen und archäologischen Zuordnungen der untersuchten Individuen in zwei soziale Gruppen (hoher/niederer Rang) wurde eine Kongruenz beobachtet. Einschränkend ist anzumerken, daß bei dieser Interpretation das dritte Cluster (mit der kleinsten Individuenzahl) über seine Zinkgehalte integriert wurde und die Argumentation der Sozialgruppen-Ernährungsgruppen-Kongruenz für dieses Cluster unabhängig von den Barium- und Strontiumkonzentrationen erfolgte. Da aber dieses dritte Cluster intern eine Differenzierung nach Barium- und Strontiumkonzentrationen zeigt, wurde bei der Beschränkung der statistischen Auswertungen auf diese beiden Elemente (wie für die aktuelle Auswertung geplant) dieser dominierende Strukturierungseffekt als entscheidend bewertet.

Das frühere Konzept bedeutete aus (hauptsächlich über Barium und Strontium definierten) Konzentrationsgruppen auf Ernährungsgruppen zu schließen und für diese in den Sozialgruppen eine Entsprechung zu finden. Hiervon ausgehend wurden die archäologisch bestimmten Sozialgruppen als zweites (alternatives) externes Gruppierungssystem neben den Histologiegruppen in die aktuellen statistischen Auswertungen integriert.

$\mathrm{Da}$ die beobachtete Kongruenz zwischen konzentrationsdefinierten Ernährungsgruppen und Sozialgruppen zwischen Männern und Frauen spezifische Unterschiede aufwies, wurde diese biologische Gruppierung (Geschlecht) ebenfalls in den neuen Auswertungen berücksichtigt. Es wurde die Interaktion zwischen den Gruppierungsfaktoren Geschlecht und Sozialgruppen ausgewertet. 
Der Fragestellungskomplex zu Weingarten kann statistisch in einer mehrfaktoriellen Varianzanalyse zusammengefaßt werden. Die drei genannten Faktoren (Geschlecht, Sozialstatus und Histologie) wurden hinsichtlich ihres Einflusses auf die Barium- und Strontiumkonzentrationen (respektive $\mathrm{Ba} / \mathrm{Ca}$ - und $\mathrm{Sr} / \mathrm{Ca}$-Werte) überprüft (zum statistischen Verfahren vgl. 3.2.3.4, S. 69). Die Ergebnisse zu diesen Fragestellungen sind in 4.1 (S. 75) aufgeführt.

Damit wurde für Weingarten exemplarisch überprüft, ob die $\mathrm{Ba} / \mathrm{Ca}$ - und $\mathrm{Sr} / \mathrm{Ca}-W e r t e$ ernährungsanzeigend interpretierbar sind oder ob die populationsinterne Variabilität beider (oder auch nur einer) Variablen diagenetische Ursachen hat. Die dargestellte Hypothesenstruktur sowie das varianzanalytische Auswertungsdesign ermöglichte außerdem die Aufklärung von Wechselwirkungen zwischen den biogenen, sozialen und diagenetischen Gruppierungsfaktoren (beschränkt auf die genannten hypothetischen Zusammenhänge).

\section{Interpopulationsvergleich diagenetischer Konzentrationsgruppen}

Mit der für Weingarten erläuterten Gegenüberstellung von in vivo vs. diagenetischen Ursachen für eine Konzentrationsvariabilität innerhalb eines Skelettkollektivs wurde zunächst nur für eine Population ein möglicher diagenetischer Einfluß überprüft. Die Frage eines systematischen Charakters diagenetischer Konzentrationsveränderungen konnte nur durch die Überprüfung weiterer Skelettkollektive beantwortet werden.

Hierfür wurden die Konzentrationsdaten der zwei weiteren historischen Bevölkerungen (Kirchheim unter Teck und Wenigumstadt) neu ausgewertet. Für beide Populationen ergaben die ursprünglichen clusteranalytischen Untersuchungen Konzentrationsgruppen, die sich hinsichtlich der beiden relevanten Elemente Barium und Strontium nicht oder bei Wenigumstadt nur für Barium unterschieden (vgl. Schutkowski 1995; Schutkowski 1998). Die ursprüngliche Hypothese der Kongruenz zwischen diesen Konzentrationsgruppen (als Ernährungsgruppen) und den Sozialgruppen macht daher einen Unterschied zwischen den Sozialgruppen hinsichtlich der $\mathrm{Ba} / \mathrm{Ca}$ - und $\mathrm{Sr} / \mathrm{Ca}-$ Werte nicht wahrscheinlich und stellt deshalb für diese beiden Bevölkerungen keine Alternative zum histologischen (diagenetischen) Gruppenkonstrukt dar. Gleiches gilt für die Geschlechtergruppen, da für diese ebenfalls keine Differenzierungen erkennbar waren. 
Für beide Populationen wurde daher jeweils nur die Histologiegruppe der gut erhaltenen Knochenproben mit jener der schlecht erhaltenen hinsichtlich Unterschieden der $\mathrm{Ba} / \mathrm{Ca}-$ und $\mathrm{Sr} / \mathrm{Ca}-W e r t e$ verglichen (zu den Ergebnissen vgl. 4.1, S. 78f).

Innerhalb dieses Untersuchungsteils war mit der vergleichenden Auswertung dreier Skelettpopulationen (Weingarten zusammen mit Kirchheim unter Teck und Wenigumstadt) die Möglichkeit gegeben, zu prüfen, ob diagenetische Veränderungen der Knochensubstanz (als histologische Veränderungen beobachtet) einen systematischen Einfluß auf die $\mathrm{Ba} / \mathrm{Ca}$ und $\mathrm{Sr} / \mathrm{Ca}$-Werte der Knochensubstanz haben. Ein mögliches populationsinternes diagenetisches Konzentrationsmuster fände seine Bestätigung in der Wiederholung (Zusammenfassung dieser Ergebnisse in 4.1, S. 80f).

\subsection{Reversibilität diagenetischer Konzentrationsveränderungen: Untersuchungen zur Probenaufreinigung}

Die statistischen Überprüfungen bezüglich möglicher diagenetischer Veränderungen der $\mathrm{Ba} / \mathrm{Ca}-$ und $\mathrm{Sr} / \mathrm{Ca}-W e r t e$ im ersten Untersuchungsteil erfolgten ausschließlich an Konzentrationsdaten von Knochenproben, die nach dem wissenschaftlich akzeptierten Aufreinigungsprinzip (unter Säureeinwirkung, zur Theorie vgl. 1.2, S. 7, zur Methode 3.2.1.3, S. 46f) vorbehandelt wurden. Alle Proben galten nach einer Überprüfung des $\mathrm{Ca} / \mathrm{P}-\mathrm{Wertes}$ (vgl. 1.2, S. 8) als hinreichend aufgereinigt und ihre Konzentrationen wurden daher ursprünglich als in vivo generiert bewertet und interpretiert. Für keine der in diesem Zusammenhang überprüften Konzentrationsdaten standen Analyseergebnisse unbehandelter Knochenproben zur Verfügung.

Hieraus resultierte folgende Situation: Ein mögliches, von der histologischen Erhaltung abhängiges (also diageneseabhängiges) Konzentrationsmuster könnte als Beleg einer unzureichenden (oder untauglichen) Aufreinigung interpretiert werden. Andererseits müssen mit veränderter histologischer Knochencharakteristik einhergehende veränderte chemische Eigenschaften des Knochenminerals hypothetisch nicht zwingend primär mit Konzentrationsveränderungen verbunden sein. Die diagenetisch veränderte Chemie des Knochenminerals könnte auch erst in Wechselwirkung mit der säureinduzierten Aufreinigung zu Konzentrationsveränderungen geführt haben.

Hieraus resultierte die Notwendigkeit eines experimentellen Vergleichs von Knochenkonzentrationen vor und nach der Aufreinigung. Es galt zunächst zu prüfen, ob es primär Kon- 
zentrationsunterschiede zwischen Knochenproben unterschiedlicher histologischer Erhaltung gibt. Wenn solche vorhanden sind, folgt die Frage, ob und inwieweit die Probenaufreinigung Einfluß auf diese Konzentrationsunterschiede hat. Mit letzterer Frage wird die Effizienz bzw. die prinzipielle Tauglichkeit der Probenaufreinigung geprüft.

Für diesen Untersuchungsaspekt wurde eine weitere Skelettpopulation aus der gleichen Zeitstellung und dem gleichen geographischen Raum wie die im ersten Versuchsteil ausgewerteten verwendet (Kirchheim am Ries, vgl. 3.1.2). Diese Bevölkerung war bisher spurenelementanalytisch nicht untersucht.

Da der Versuch einen prinzipiellen Kontrollcharakter hatte, wurden biologische Parameter, die unabhängig von Ernährung oder Diagenese primär die Knochenkonzentrationen beeinflussen bzw. auch zu variablem Verhalten der Knochen unter der Liegezeit und während der Probenaufreinigung führen könnten, möglichst konstant gehalten.

Um altersabhängige Veränderungen der Mineraleigenschaften infolge eines geänderten Hormonstatus gering zu halten, sind für diesen Versuch nur Knochenproben von Frauen in einem morphologisch bestimmten Alter zwischen 20 und 40 Jahren und Männer zwischen 20 und 50 Jahren genutzt worden. Eine ausschließliche Beschränkung auf Männer war infolge der weiteren Vorgaben für die Knochenproben nicht möglich, da der Stichprobenumfang dann zu gering geworden wäre.

Zur Vermeidung intraindividueller Konzentrationsunterschiede (vgl. Tanaka et al. 1981; Brätter et al. 1977) wurden ausschließlich Femurproben genutzt (dies war bei den bisher erwähnten Skelettkollektiven nicht konsequent möglich, vgl. 3.2.1.1).

Als weiteres Auswahlkriterium wurde versucht, Proben von Skelettfunden zu verwenden, deren Bestattungen möglichst gleichmäßig auf dem Gräberfeld verteilt waren (vgl. 3.1.2, Abbildung 4). Hiermit sollte vermieden werden, daß mögliche lokale Besonderheiten des Liegemilieus, die zu spezifischen Veränderungen des Knochensubstanz führen könnten, in der Stichprobe überrepräsentiert sind.

Mit diesen Vorgaben wurden zur Erfassung der wahrscheinlichen diagenesebedingten Konzentrationsveränderungen wieder Proben aus den beiden histologischen Gruppen (gut/schlecht) ausgewählt.

Die Aufreinigungsüberprüfung erfolgte als Vergleich zwischen vier Varianten der Probenvorbereitung, d.h. es wurden für jede der untersuchten Knochenproben die Elementkonzentrationen einer Teilprobe unbehandelt und drei weiterer Teilproben nach verschiedenen 
Modifikationen der Probenaufreinigung analysiert. Es resultieren 4 Probensätze bzw. für jede Knochenprobe ein Probenquartett, daß sich auf diese vier Probensätze verteilt.

Die Varianten der Probenvorbereitung können zusammenfassend wie folgt beschrieben werden:

- Proben unbehandelt: es erfolgte keinerlei Probenaufreinigung; nur etwaige Sedimentsowie Spongiosareste wurden entfernt

- Proben geätzt: Säurebehandlung (detailliert in 3.2.1.6, S. 49f)

- Proben oberflächenbehandelt: sowohl periost- als markraumseitig wurde die Oberflächenschicht mechanisch entfernt (detailliert in 3.2.1.6, S. 49)

- oberflächenbehandelt und geätzt: zunächst periost- und markraumseitig die Oberfläche mechanisch entfernt und zusätzlich geätzt

Die Variante der Säurebehandlung entspricht der Probenaufreinigung, wie sie auch für die im ersten Untersuchungsteil neu ausgewerteten Skelettserien verwendet wurde. Die in 3.2.1.6 (S. 49f) dargelegten Modifikationen bedeuten gegenüber der früheren Methode nur eine graduelle Optimierung für einen effektiveren Lösungsprozeß, stellen aber keine prinzipielle Änderung dar. Ein dadurch eventuell verbesserter Aufreinigungseffekt könnte in den Auswertungen den früheren Untersuchungen gegenübergestellt werden. Die hiermit verwendete säureinduzierte Aufreinigung ist dem allgemein anerkannten Aufreinigungsprinzip analog, daß auf der Annahme differentieller Löslichkeiten von nativem und diagenetisch verändertem Knochenmineral beruht (vgl. 1.2, S. 7).

Werden mit der chemischen Methode des Ätzens chemische Veränderungen innerhalb der gesamten Knochenkompakta erfaßt, ist die Oberflächenbehandlung ausgerichtet auf Konzentrationsveränderungen entsprechend eines Diffusionsgradienten (vgl. z.B. Lambert et al. 1983; Williams 1988; Reiche et al. 1999) für Elemente innerhalb des Knochens. Mit der Entfernung der oberflächennahen Schicht wird jener Bereich der Probe, der am schnellsten durch Elementeinträge aus dem Boden beeinflußt werden kann, entfernt. Da Knochenproben generell auch von Femurfragmenten stammen können und daher auch die Markhöhle mit Sediment gefüllt sein kann, ist eine Entfernung der markraumseitigen Oberfläche ebenfalls zweckmäßig. Diese Oberflächenentfernung entspricht den ersten Maßnahmen zur Probenbehandlung im Diagenesekontext (vgl. 1.2, S. 7), die aber auch oft zusammen mit 
der chemischen Aufreinigung verwendet wird. Deshalb erfolgte in der letzten Aufreinigungsvariante eine Kombination aus chemischer und mechanischer Probenaufreinigung.

Durch das dargestellte Versuchsdesign der vier Probenaufreinigungsvarianten sind folgen-

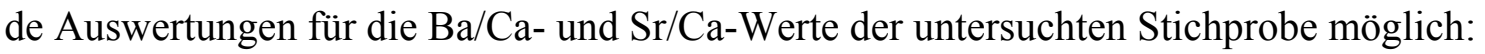

- Die Konzentrationsdaten des unbehandelten Probensatzes erlauben über den Vergleich der beiden Histologiegruppen (gut/schlecht) ein Aussage (unter Ausschluß einer möglichen artifiziellen Beeinflussung durch Probenaufreinigung!), ob diagenetische Veränderungen der Knochensubstanz zu Unterschieden der zur Ernährungsrekonstruktion verwendeten $\mathrm{Ba} / \mathrm{Ca}$ - und $\mathrm{Sr} / \mathrm{Ca}-W e r t e$ führen (Ergebnisse in 4.2, S. 82ff).

- Sollte ein diageneseabhängiges Konzentrationsmuster im unbehandelten Probensatz vorhanden sein, kann durch Vergleich der Histologiegruppen innerhalb der Probensätze nach den verschiedenen Methoden der Vorbehandlung überprüft werden, ob dieses Muster erhalten bleibt bzw. wie es sich verändert. Hiermit würde Tauglichkeit und Effizienz der Aufreinigungsmethoden allgemein erfaßt werden.

- Unabhängig, ob und wie sich ein mögliches diageneseabhängiges Konzentrationsmuster ändert, kann geprüft werden, ob die $\mathrm{Ba} / \mathrm{Ca}$ - und $\mathrm{Sr} / \mathrm{Ca}-W e r t e$ der beiden Histologiegruppen (gut/schlecht) durch die verschiedenen Aufreinigungsmethoden differentiell beeinflußt werden. Hierdurch können Aussagen zur Theorie der Aufreinigung abgeleitet werden, die eine differentielle Beeinflussung diagenetisch unterschiedlich veränderter Knochenproben annimmt.

Hierbei bilden die beiden letztgenannten Auswertungsoptionen eine inhaltliche Einheit bezüglich der Überprüfung der Aufreinigungsmethoden. Diese werden im Ergebnisteil zusammengefaßt ausgewertet (vgl. 4.2, S. 84ff).

Das Auswertungsdesign dieses Teilversuches entspricht einer Meßwiederholungsuntersuchung. Es erfolgen Vergleiche der Konzentrationsdaten von unbehandelten mit nach drei verschiedenen Aufreinigungsmethoden vorbereiteten Knochenproben. Zusätzlich werden die beiden Histologiegruppen jeweils miteinander verglichen. Die Überprüfung auf eine differentielle Aufreinigungswirkung auf die Histologiegruppen beschreibt die Interaktion zwischen Histologiegruppe und Behandlungsmethode. Das verwendete statistische Verfahren wird in 3.2.3.4 (S. 69f) vorgestellt.

Da für diesen Versuch jede Knochenprobe in mehrere Probenstücke unterteilt wurde, ist grundsätzlich die Möglichkeit von Konzentrationsunterschieden innerhalb des untersuch- 
ten Gesamtstückes zu berücksichtigen. Die experimentelle Überprüfung einer solchen etwaigen intraossären Variabilität war daher erforderlich. Diese wird infolge ihrer unabhängig von diesem Teilversuch erweiterten Untersuchungsrelevanz als eigenständiger Teilversuch in 2.5 vorgestellt.

\subsection{Das Problem einer zuverlässigen Diagnose diagenetischer Veränderungen: Kontrollkriterien im Test}

Wie schon für den Versuchsteil zur Überprüfung der Probenaufreinigung bildet auch für diesen Untersuchungsaspekt die Neuauswertung der früheren Untersuchungen (vgl. 2.1) den Ausgangspunkt. Die ursprünglichen Auswertungen jener Untersuchungen stützten sich auf die Überprüfung der Knochenproben anhand eines in der Literatur allgemein anerkannten Kontrollkriteriums (Ca/P-Wert, ausführlich in diesem Kapitel, S. 28f). Dieser Prüfung zufolge wurden die Knochenproben nach der Probenaufreinigung als von diagenetischem Knochenmaterial hinreichend befreit eingeschätzt, entsprechend ausgewertet und die $\mathrm{Ba} / \mathrm{Ca}$ und $\mathrm{Sr} / \mathrm{Ca}-W e r t e$ als in vivo generierte, ernährungsanzeigende Konzentrationsverhältnisse interpretiert.

Aus dem (begründeten) Verdacht einer unzureichenden Aufreinigung trotz dieser Beurteilung resultiert die Fragestellung für diesen Teilversuch:

Wie zuverlässig sind die verwendeten Kontrollkriterien zur Charakterisierung diagenetischer Veränderungen des Knochenminerals?

Ausgangspunkt aller bisherigen Teiluntersuchungen war die histologische Zuordnung, d.h. es wurde zunächst ausschließlich die lichtmikroskopische Beurteilung der Knochenbinnenstruktur zur Einteilung von Knochenproben in Gruppen unterschiedlicher diagenetischer Veränderung (gut/schlecht) verwendet (vgl. 2.1, S. 12ff). Diese Gruppen wurden anschließend hinsichtlich möglicher Unterschiede ihrer $\mathrm{Ba} / \mathrm{Ca}$ - und $\mathrm{Sr} / \mathrm{Ca}-W e r t e$ überprüft. Vorhandene Unterschiede sind als diagenetische Konzentrationsveränderungen des Knochenminerals zu verstehen. Kontrollkriterien, die zwischen unterschiedlichen Graden chemischer diagenetischer Veränderungen des Knochenminerals differenzieren sollen, müßten im Falle solcher Konzentrationsunterschiede zwischen den beiden histologischen Gruppen (gut/schlecht) differenzieren.

Nach der Feststellung solcher Konzentrationsunterschiede zwischen den Histologiegruppen (dieser Vorgriff auf Ergebnisse ist für das Verständnis der weiteren Vorgehensweise 
unumgänglich, vgl. 4.3) wurde für die im folgenden aufgeführten Kontrollkriterien dieses Differenzierungsvermögen überprüft. Hierfür wurden sowohl die Daten der bereits früher untersuchten Populationen (Weingarten, Kirchheim unter Teck, Wenigumstadt) als auch die im Probenaufreinigungsversuch verwendete Stichprobe von Kirchheim am Ries entsprechend statistisch ausgewertet.

\section{Der ernährungsbedingte Barium-Strontium-Zusammenhang innerhalb einer Skelettpopulation}

Begonnen wird mit einem Kriterium, das sich direkt auf die beiden ernährungsrelevanten Elemente Barium und Strontium bezieht. Dieses Prüfkriterium stellt den Versuch dar, ohne externe Kontrolle, nur anhand der eigentlich untersuchten Elemente, diagenetische Effekte zu erfassen (Burton et al. 1999).

Die Erklärung für das Kontrollprinzip erfolgt über die gleichgerichtete Abnahme beider Konzentrationsquotienten in der Nahrungskette (vgl. 1.1, S. 2f). Hieraus resultiert für die verschiedenen pflanzlichen und tierischen Nahrungskomponenten einer aus einem Nahrungsnetz zusammengestellten Mischkost ein enger Zusammenhang zwischen den $\mathrm{Ba} / \mathrm{Ca}-$ und $\mathrm{Sr} / \mathrm{Ca}-W e r t e n$. Ist in einer Nahrungskomponente im Vergleich zu einer weiteren das $\mathrm{Ba} / \mathrm{Ca}-$ Verhältnis geringer, so gilt das gleiche auch für das $\mathrm{Sr} / \mathrm{Ca}-\mathrm{Verhältnis.} \mathrm{Dies} \mathrm{trifft}$ unabhängig davon zu, daß der Diskriminierungsprozeß innerhalb der Nahrungskette für Barium stärker verläuft und daraus eine überproportionale Abnahme für dieses Element erfolgt (Gilbert et al. 1994; Elias et al. 1982). Hieraus folgt bei einem Vergleich zweier Individuen, die sich innerhalb dieses Nahrungsnetzes ernähren, für jenes mit dem geringeren nahrungsbedingten $\mathrm{Ba} / \mathrm{Ca}$-Verhältnis im Knochenmineral auch das geringere $\mathrm{Sr} / \mathrm{Ca}$ Verhältnis und dies unabhängig von der in 1.1 (S. 4) erläuterten Einschränkung für eine direkte Übersetzung von Knochenkonzentrationen in Nahrungszusammensetzung. Innerhalb einer Population wird entsprechend (weniger als erste denn als hinreichende Näherung) eine Korrelation zwischen den $\mathrm{Ba} / \mathrm{Ca}$ - und $\mathrm{Sr} / \mathrm{Ca}-W e r t e n$ der Knochenproben vorausgesagt und empirisch bestätigt. Wichtig ist hierbei die Beschränkung auf eine Bevölkerung deren Ernährung auf terrestrischen Nahrungsressourcen basiert (vgl. 1.1, S 5). Desweiteren ist auf den regional variablen geochemischen Hintegrund für Barium und Strontium im Falle verschiedener Nahrungsnetzen hinzuweisen. Der Zusammenhang zwischen Barium und Strontium besteht nur innerhalb einer Bevölkerung. Zwei Individuen zweier Bevölkerungen aus verschiedenen Nahrungsnetzen können bei gleich hohen $\mathrm{Sr} / \mathrm{Ca}-$ 
Werte davon unabhängig unterschiedliche hohe $\mathrm{Ba} / \mathrm{Ca}$-Werte aufweisen (zum BariumStrontium-Zusammenhang vgl. Burton \& Price 1990a; Burton et al. 1999).

Die Argumentation der genannten Autoren hinsichtlich der Kontrollfunktion dieses Zusammenhanges geht dahin, daß, im Falle diagenetischer Veränderungen der Knochenkonzentrationen von Barium und Strontium, diese unsystematisch sind und die Korrelation zwischen $\mathrm{Ba} / \mathrm{Ca}$ - und $\mathrm{Sr} / \mathrm{Ca}-W e r t e n$ zunehmend unwahrscheinlicher bzw. der Korrelationskoeffizient kleiner wird.

Alle in der vorliegenden Arbeit untersuchten Bevölkerungen hatten terrestrische Ernährungsgrundlagen (vgl. geographische und archäologische Einordnung in 3.1). Für die Knochenproben sollte innerhalb der Bevölkerungen der charakteristische Zusammenhang der $\mathrm{Ba} / \mathrm{Ca}$ - und $\mathrm{Sr} / \mathrm{Ca}-W e r t e$ vorhanden sein und das genannte Kontrollprinzip kann auf die beiden Histologiegruppen übertragen werden. Die Vorgehensweise besteht dann in einem Vergleich der gruppeninternen Korrelationskoeffizienten. Diagenetische Veränderungen der $\mathrm{Ba} / \mathrm{Ca}-$ bzw. Sr/Ca-Werte verringern den Korrelationskoeffizienten. Sind die histologisch schlecht erhaltenen Knochenproben diagenetisch stärker verändert (einschließlich einer Beeinflussung der $\mathrm{Ba} / \mathrm{Ca}$ - und $\mathrm{Sr} / \mathrm{Ca}-W e r t e)$ als die gut erhaltenen, sollte ihr Korrelationskoeffizient kleiner sein. Die Ergebnisse dieser Korrelationsvergleiche für alle Populationen (Kirchheim unter Teck, Wenigumstadt, Weingarten, Kirchheim am Ries) sind in 4.3 (S. 88ff) zusammengestellt.

\section{Das Ca/P-Verhältnis des Knochenminerals}

Der Quotient aus der Calcium- und Phosphorkonzentration $(\mathrm{Ca} / \mathrm{P}$, innerhalb dieser Arbeit konsequent als Massenrelation verwendet) einer Knochenprobe gilt als ein wichtiger (oft verwendeter bzw. zitierter) mineralchemischer Parameter zur Charakterisierung des Erhaltungszustandes. Aus der Stöchiometrie der Hauptkomponente des Knochenminerals $\left[\mathrm{Ca}_{10}\left(\mathrm{PO}_{4}\right)_{6}(\mathrm{OH})_{2}\right.$, stöchiometrisches Verhältnis von $\left.\mathrm{Ca}: \mathrm{P}=10: 6\right]$ resultiert als theoretische Vorgabe für die Massenrelation ein Wert von 2,16. Durch empirische rezente Daten wurde dieser theoretische chemische Wert um die biologische Variabilität des Knochenmi-

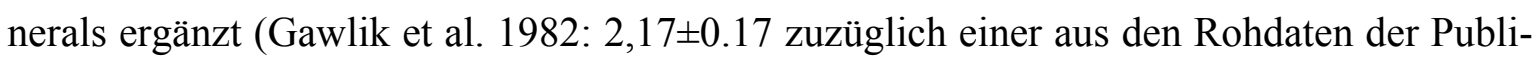
kation berechneten Spannweite von 2,0-2,36). Damit steht prinzipiell ein nativer Referenzwert zur Verfügung, der einer biochemischen (physiologischen) Konstanten entspricht. Das bedeutet insbesondere, daß dieses $\mathrm{Ca} / \mathrm{P}-$ Verhältnis ernährungsunabhängig ist 
und von daher ein universelles Kriterium zur Kontrolle des nativen Charakters (menschlichen) Knochenminerals sein sollte. Es ist aber prinzipiell zu berücksichtigen, daß Knochenmineral eine mehrphasige Zusammensetzung (verschiedene Ca-P-Verbindungen) hat und das spezifische $\mathrm{Ca} / \mathrm{P}-$ Verhältnis aus der charakteristischen Komposition dieser Phasen resultiert (Driessens \& Verbeeck 1980).

Im Zusammenhang mit diagenetischen Veränderungen der Mineralkomposition des Knochens werden Abweichungen des Quotienten von seinem nativen Wert erwartet, wobei ein systematischer Charakter der Veränderung diskutiert wird. Einerseits können Umkristallisationsprozesse zu veränderten Relationen der verschiedenen Ca-P-Verbindungen der Mineralmatrix führen. Hierbei werden unabhängig vom möglichen multidirektionalen Charakter dieser Prozesse (Pate \& Brown 1985) bei spezifischem Liegemilieu Verringerungen des $\mathrm{Ca} / \mathrm{P}-$ Wertes erwartet (vgl. Herrmann \& Newesely 1982; Herrmann \& Nitsch 1984). Andererseits sind infolge von Calciumcarbonateinträgen erhöhte $\mathrm{Ca} / \mathrm{P}-\mathrm{Werte}$ möglich (z.B. White \& Hannus 1983; Sillen 1989). [Es sei an dieser Stelle angemerkt, daß das chemische Aufreinigungsprinzip auf der erhöhten Löslichkeit dieser veränderten Ca-PVerbindungen bzw. der fremdeingetragenen Verbindungen beruht (vgl. 1.2, S. 7)]

Das Ca/P-Kontrollkriterium wurde für die früheren Auswertungen (Weingarten, Kirchheim unter Teck und Wenigumstadt) zwar genutzt, aber dabei war nicht eine serieninterne Differenzierung nach histologischer Erhaltung relevant, sondern die Werte wurden mit den rezenten Referenzwerten verglichen und auf dieser Grundlage als hinreichend erhalten bzw. aufgereinigt bewertet.

Im Rahmen der jetzigen Untersuchung wurde spezifisch geprüft, ob es Unterschiede der $\mathrm{Ca} / \mathrm{P}-$ Werte zwischen den beiden Gruppen mit diagenetisch unterschiedlich veränderter Histologie (gut/schlecht) gibt. Hervorzuheben ist, daß dieser Vergleich bei der im Probenaufreinigungsversuch analysierten Stichprobe von Kirchheim am Ries für den unbehandelten Probensatz durchgeführt wurde. Etwaige diagenetische Veränderungen des $\mathrm{Ca} / \mathrm{P}-$ Verhältnisses müßten zwischen den beiden Histologiegruppen hierbei am deutlichsten zu erfassen sein.

Die Ergebnisse der Ca/P-Vergleiche der Histologiegruppen aller ausgewerteten Populationen (Kirchheim unter Teck, Wenigumstadt, Weingarten, Kirchheim am Ries) sind in 4.3 (S. 92ff) aufgeführt. 


\section{Magnesium (und Natrium) als knochenimmanente Elemente}

Magnesium wurde als ein weiteres Kontrollkriterium im Hinblick auf seine physiologische Relevanz verwendet. Infolge dieser Bedeutung in vivo ist es in relativ hoher Konzentration im Knochenmineral vorhanden und wird hauptsächlich in einer spezifischen und insbesondere leichtlöslichen Mineralkomponente gebunden (Driessens 1980). Dies bedeutet, daß dieses Element während der Bodenlagerung des Knochenminerals forciert aus dem Knochen herausgelöst werden kann (vgl. z.B. Reiche et al. 1999).

Magnesium steht infolge der spezifischen Einbindung in das Knochenmineral nicht in direkter Korrespondenz mit den wahrscheinlich hauptsächlich in das Hydroxylapatit integrierten Elementen Barium und Strontium, es kann aber als sensitiver Marker für einen Vergleich unterschiedlich starker diagenetischer Veränderungen von Knochenproben verwendet werden.

Zusätzlich stehen auch für Magnesium rezente Referenzdaten zur Verfügung (vgl. z.B. (D'Haese et al. 1999; Utsumi et al. 1999; Mongiorgi et al. 1990). Trotz einer homöostatischen Kontrolle (vgl. 1.1, S. 4) ist eine hohe interindividuelle Variabilität festzustellen. Als Referenz sind daher nur die Größenordnungen zu verwenden.

Im Rahmen der früheren Untersuchungen wurden keine Magnesiumkonzentrationen bestimmt. Entsprechende Auswertungen waren für diese Skelettserien daher nicht möglich.

In der vorliegenden Untersuchung Arbeit wurden für die Stichprobe von Kirchheim am Ries die Magnesiumkonzentrationen gemessen. Dies erfolgte insbesondere für alle vier Probensätze des Aufreinigungsversuches. Hiermit bestand die Möglichkeit, spezifische Aufreinigungseffekte über Konzentrationsveränderungen dieses Elementes zu charakterisieren (vgl. 2.2).

Die Magnesiumgehalte (als Mg/Ca-Werte) beider Histologiegruppen (gut/schlecht) wurden somit sowohl für unbehandelte Proben als auch für die Probensätze nach den verschiedenen Aufreinigungsvarianten verglichen. Die Ergebnisse zu den Magnesiumanalysen sind in 4.3 (S. 94ff) aufgeführt.

Natrium wurde ursprünglich nicht mit in das Untersuchungskonzept eingebunden. Dies begründete sich aus der Ambivalenz einer einerseits möglichen Anreicherung unter der Liegezeit infolge der Kosubstitution von $\mathrm{Na}^{+}$und $\mathrm{CO}_{3}{ }^{2-}$ für $\mathrm{Ca}^{2+}$ und $\mathrm{PO}_{4}{ }^{3-}$ (Mechanismus nach Chickerur et al. 1980; zur Natriumeinbindung im nativen Knochenmineral vgl. Driessens 1980) und andererseits der beobachteten und mit hoher Mobilität begründeten 
Abreicherung (vgl. z.B. Reiche et al. 1999). Eine eindeutige Kontrollfunktion war hiernach empirisch fraglich. Nachdem jedoch die mikroanalytisch Untersuchung einer Knochenprobe (vgl. 2.4) eine spezifische Charakteristik für Natrium ergab, wurden für eine kleine Stichprobe für Kirchheim am Ries die Natriumgehalte der Knochenproben bestimmt. Die Ergebnisse sind als $\mathrm{Na} / \mathrm{Ca}-W e r t e$ in 4.4 .4 dokumentiert.

\section{Aluminium als kontaminationsanzeigendes Element}

Aluminium ist unter normalen physiologischen Bedingungen nicht bzw. nur in sehr geringen Konzentrationen im Knochenmineral enthalten (vgl. z.B. D'Haese et al. 1999). Im Boden meist in erhöhten Konzentrationen vorhanden und gleichzeitig hoch mobil, ist es ein hervorragender Marker für ein in das Knochenmineral leicht diffundierendes Element (vgl. z.B. Lambert et al. 1985; Price 1989 Reiche et al. 1999).

Die entsprechenden Vergleiche der Histologiegruppen konnten für dieses Element ebenfalls nur für Kirchheim am Ries durchgeführt werden. Hier aber wieder sowohl für die unbehandelten Proben als auch für die differentiell vorgereinigten Probensätze (vgl. 2.2). Die Ergebnisse der Aluminiumanalysen (als Al/Ca-Werte) sind in 4.3 (S. 97ff) dokumentiert.

\section{Masseverlust der Knochenproben bei der chemischen Probenaufreinigung}

Mit diesem Kontrollkriterium werden Daten aus der Probenaufreinigung ausgewertet. Der relative Masseverlust einer Knochenprobe während der chemischen Aufreinigung ist in Verbindung mit dem Aufreinigungsprinzip zu verstehen (vgl. 1.2, S. 7 bzw. in diesem Kapitel zum Ca/P, S. 28f). Ausgangspunkt ist, daß durch eine säureinduzierte Aufreinigung verändertes Mineral infolge seiner erhöhten Löslichkeit forciert aus einer Knochenprobe entfernt wird. Unter der Annahme, daß histologisch schlecht erhaltene Knochenproben stärker diagenetisch verändert sind als gut erhaltene, sollten diese Knochenproben einen gegenüber gut erhaltenen Proben höheren Masseverlust durch die Säureeinwirkung zeigen. Da durch die Säure auch natives Material herausgelöst wird und bei den früheren Untersuchungen auch die Säurebehandlungsdauer nicht exakt definiert war, wurde dieses Kriterium nur unter Vorbehalt quantitativ ausgewertet (zur detaillierten Methode der chemischen Aufreinigung durch Ätzen vgl. 3.2.1.3, S. 46f). 
Die Resultate dieses Masseverlustes liefern keine Information über den Zustand des Knochens nach der Aufreinigung (inwieweit die Aufreinigung erfolgreich war), sondern nur eine Abschätzung über den Masseanteil wahrscheinlich diagenetisch veränderter Mineralbestandteile der Knochenprobe vor der Aufreinigung.

Bezüglich dieses charakteristischen Masseverlustes konnten die beiden definierten Histologiegruppen für drei (Wenigumstadt, Weingarten, Kirchheim am Ries) der vier innerhalb dieser Arbeit ausgewerteten Skelettpopulationen verglichen werden. Die Ergebnisse sind in 4.3 (S. 100ff) aufgelistet.

\section{Masseverlust der Knochenprobe beim Veraschen}

Mit diesem Masseverlust während eines Verbrennungsvorganges im Rahmen der Probenvorbereitung (vgl. 3.2.1.4, S. 47f) wird der noch in der Knochensubstanz enthaltene (in vivo bedingte) Organikanteil abgeschätzt, da dieser durch die definierten Parameter der Verbrennung annähernd vollständig oxidiert wird.

Die organische Matrix der Knochensubstanz (90 \% Kollagen, vgl. Hedges et al. 1995) ist in zweierlei Hinsicht mit diagenetischen Veränderungen der Knochensubstanz assoziiert. Einerseits kann angenommen werden, daß durch das organische Gefüge das Knochenmineral primär vor diagenetischen Veränderungen geschützt wird (Sillen 1989; Pate et al. 1989). Andererseits ist genau diese organische Komponente als Nahrungsressource von Mikroorganismen bedroht. Führt schon deren primäre Einwirkung zur mineralischen Umbildung mit möglichen Konzentrationsveränderungen (vgl. Grupe et al. 1993; Grupe \& Piepenbrink 1988), sind in ihrer Folge bodenchemische Wechselwirkungen durch die fehlende Schutzwirkung sehr wahrscheinlich erleichtert möglich.

Da die histologische Bewertung der Knochenproben auf charakteristischen Veränderungen der Knochenbinnenstruktur beruht und diese mit mikrobiellem Einfluß in Verbindung gebracht werden (vgl. 2.1, S. 12ff), ist eine Differenzierung der beiden Histologiegruppen (gut/schlecht) durch dieses Kontrollkriterium des Veraschungsverlustes sehr naheliegend.

Hinzu kommt, daß sich der Veraschungsverlust als Maß für den Organikanteil der Knochensubstanz durch einen wichtigen Vorteil auszeichnet. Wie für das $\mathrm{Ca} / \mathrm{P}-$ Verhältnis (vgl. S. 28f), steht für den organischen Masseanteil ein Referenzwert für natives Knochenmaterial zur Verfügung (30\%, vgl. Leblond \& Weinstock 1976). Hiermit ist zusätzlich 
zu einer relativen Unterscheidung zwischen Knochenproben unterschiedlicher Erhaltung ein absoluter Bezug zu rezentem Knochenmaterial möglich.

Die Ergebnisse der diesbezüglichen Vergleiche der Histologiegruppen für Wenigumstadt, Weingarten und Kirchheim am Ries sind in 4.3 (S. 102ff) aufgeführt.

\subsection{Diagenetische Veränderungen des Knochenminerals im Detail: Mikroanalytische Untersuchungen eines Knochenquerschnittes}

Die Vergleiche der Elementkonzentrationen zwischen verschiedenen Knochenproben sowie die Auswertung möglicher diageneseanzeigender Charakteristika wurden mit dem folgenden Teilversuch ergänzt. Es erfolgte die Auswertung spezifischer Konzentrationsverteilungen innerhalb der Mikrostruktur einer Knochenprobe.

Die Nutzung mikroanalytischer Techniken wird im Rahmen von Spurenelementuntersuchungen an bodengelagertem Skelettmaterial seit längerem empfohlen, aber nur mit "...varying degrees of success..." realisiert (vgl. Überblick und das genannte Zitat in Sandford \& Weaver 2000, S. 341). Im Rahmen der vorliegenden Arbeit konnte vom Fortschritt der apparativen Technik (und insbesondere von der Kompetenz von Dr. Klaus Simon und Dr. Andreas Kronz, Abteilung Geochemie des Göttinger Zentrums Geowissenschaften) profitiert werden. Die Verwendung modernster Analysetechnik (EPMA und LA-ICP-TOFMS, vgl. 3.2.2.2) ermöglichte hochauflösende Analysen von Hauptelementen des Knochenminerals sowie die Erstellung radialer Konzentrationsprofile für Spurenelemente innerhalb eines Kompaktaquerschnittes.

Für diesen Versuch wurde eine histologisch als mittel erhalten eingeordnete Probe gewählt, d.h. eine Probe aus jener Erhaltungskategorie, die von den statistischen Vergleichen der Knochenproben in den vorangegangenen Versuchsteilen ausgeschlossen wurde (vgl. 2.1, S. 12ff; analysiert wurde die dort in Abbildung 2 dargestellte Knochenprobe KR 261 von Kirchheim am Ries). Es wurde gezielt eine Probe mit einer variierenden Erhaltungscharakteristik aus intakter (histologisch gut) und veränderter (histologisch schlecht) Knochenbinnenstruktur ausgewählt, da es das Ziel dieser Untersuchung war, innerhalb der Kompakta spezifische Elementverteilungsmuster in Abhängigkeit von einem solchen Erhaltungsmosaik zu dokumentieren.

In den Analysen wurden die Matrixelemente Calcium und Phosphor (insbesondere bezüglich ihrer $\mathrm{Ca} / \mathrm{P}$-Charakteristik) sowie die in den vorangegangen Untersuchungsteilen analy- 
sierten Elemente Magnesium und Natrium im Kontext diageneseanzeigender Kontrollkriterien erfaßt. Zusätzlich hierzu konnte Schwefel analysiert werden, ein Element, das infolge technischer Limitierungen bei den Pauschalanalysen der Gesamtknochenproben nicht bestimmt werden konnte (vgl. 3.2.2.1). Dies eröffnete die Möglichkeit einer zusätzliche Diagenesecharakterisierung.

Im Kontext der primären Fragestellung bezüglich möglicher diagenetischer Veränderungen der eigentlich als ernährungsanzeigend ausgewerteten Elementkonzentrationen von Barium und Strontium wurden für diese beiden Elemente radiale Konzentrationsprofile erfaßt. Zur Auswertung als rein diagenetischer Marker wurde ein solches Profil auch für das Element Uran erstellt (vgl. Millard \& Hedges 1995).

Die Ergebnisse dieses Teilversuches bieten durch die präzise (elektronenmikroskopische) Zuordnung der diagenetischen Morphologie der Knochenbinnenstruktur zu einer spezifischen Charakteristik der Elementverteilung die Möglichkeit einer erweiterten Interpretation der pauschalanalytischen Vergleiche ganzer Knochenproben der vorangegangen Untersuchungsteile. Die dort ausgewerteten Teilaspekte diagenetischer Prozesse können hier in einen Zusammenhang gestellt werden, auch wenn diese kombinierte Betrachtung den Rahmen einer erweiterten Phänomenologie diagenetischer Veränderungen des Knochenminerals nicht überschreitet.

\subsection{Ein diageneseunabhängiges Problem: Untersuchung zur intraossären Variabilität von Elementkonzentrationen}

Wie bereits im Kapitel zum Probenaufreinigungsversuch erwähnt (vgl. 2.2), ging dieser Versuch aus einem Kontrollversuch zur Probenaufreinigung hervor. Die vergleichenden Analysen jeweils mehrerer Teilproben einer Femurdiaphyse erforderten die Überprüfung auf eine mögliche intraossäre Variabilität, da eine solche den zu überprüfenden Aufreinigungseffekt hätte maskieren können.

Die diesbezügliche ursprüngliche Fragestellung war auf die Konzentrationsvariabilität innerhalb eines anterioren Femurstreifens beschränkt, da dies die standardisierte Probenentnahmestelle für die untersuchten Knochenproben ist (vgl. 3.2.1.1).

Die bisherigen Untersuchungen zur intraindividuellen Konzentrationsvariabilität waren meist auf die Frage von Konzentrationsunterschieden zwischen verschiedenen Skelettelementen beschränkt oder erfaßten nur longitudinale Konzentrationsunterschiede innerhalb 
eines Langknochens (Tanaka et al. 1981, Grupe 1988; Brätter et al. 1977). Frühere Untersuchungsergebnisse charakteristischer Mineraldichteverteilungen innerhalb des Femurs (Amtmann \& Schmitt 1968) bzw. funktionsmorphologische Anpassungen der Femurkompakta (Amtmann \& Doden 1981) machen aber eine physiologisch variable Mineralkomposition wahrscheinlich. Diese Untersuchungen sowie die Arbeit von Schug (1997), die für ein bodengelagertes Femur eine horizontale Konzentrationsvariabilität aufzeigte, wurden zum Anlaß genommen, die Untersuchung der intraossären Variabilität von Elementkonzentrationen auf einen längeren Diaphysenabschnitt in seiner Gesamtheit (ohne Bereich der Linea aspera) auszuweiten.

Es wurde ein Abschnitt von ca. $10 \mathrm{~cm}$ aus der Diaphysenmitte eines rezenten Femurs (Material detailliert in 3.1.3) untersucht. Das Diaphysenstück wurde longitudinal in fünf Ebenen und in jeder Ebene horizontal in acht Teilstücke (zur Probenvorbereitung vgl. 3.2.1.8) untergliedert.

Mit dieser Probenaufteilung konnte die Konzentrationsvariabilität eines Diaphysenabschnittes über 40 Teilproben $(5 \times 8)$ mit definierter horizontaler und longitudinaler $\mathrm{Zu}$ ordnung der Teilproben "kartiert" werden. Für die Teilproben wurde das auch in den anderen Teiluntersuchungen dieser Arbeit analysierte Elementspektrum (Ca, P, Ba, Sr, Mg, Al sowie für acht Teilproben Na) gemessen. Im Rahmen der Probenvorbereitung wurde für das rezente Knochenmaterial ebenfalls der Veraschungsverlust dokumentiert (vgl. 2.3, S. 32f). Diese Daten standen außer für die Fragestellung dieses Versuchsteils auch zum Vergleich mit dem bodengelagerten Skelettmaterial aus den anderen Teiluntersuchungen zur Verfügung.

Als ein Abschätzungexperiment zur Elementverteilung innerhalb des Femurs geplant, wurde im Rahmen dieser Arbeit nur eine Femurdiaphyse untersucht. Systematische Verteilungsmuster nahrungsanzeigender Elemente (Barium und Strontium) wären ein Hinweis auf physiologische Überzeichnungen des Nahrungssignals. Daraus würde sich die Notwendigkeit weiterer Überprüfungen zur intraossären Variabilität von Spurenelementkonzentrationen ableiten. Eine physiologische Abhängigkeit der Elementverteilung innerhalb eines Femurs wäre bei einer Interpretation der Elementkonzentrationen von bodengelagertem Skelettmaterial im Kontext der Ernährungsrekonstruktion unbedingt zu berücksichtigen. 
Dieser Aspekt ist von den anderen Teilversuchen dieser Arbeit zwar losgelöst, gliedert sich aus den genannten Gründen jedoch in die Validitätserwägungen zur Spurenelementanalytik ein. Die Ergebnisse sind in 4.5 dargestellt. 


\section{$2.6 \quad$ Überblick zu den Fragestellungen}

Aus den Kapiteln 2.1 bis 2.5 können folgende Fragestellungen zusammengefaßt werden. Die einzelnen Fragen sind den versuchsbeschreibenden Kapiteln zugeordnet. Zusätzlich werden jeweils im Anschluß der Frage die zugehörigen Ergebniskapitel angegeben.

\section{Fragestellungen zum Versuchsteil 2.1:}

- Gibt es populationsintern für die $\mathrm{Ba} / \mathrm{Ca}$ - und $\mathrm{Sr} / \mathrm{Ca}$-Werte von Knochenproben verschiedener Individuen Unterschiede zwischen den Geschlechtergruppen oder/und zwischen den Sozialgruppen oder/und zwischen Gruppen, die über den histologischen Erhaltungszustand der Knochensubstanz definiert wurden? (4.1, S. 75ff)

- Gibt es innerhalb verschiedener Skelettpopulationen ein vom histologischen Erhaltungszustand der Knochenproben abhängiges Muster der $\mathrm{Ba} / \mathrm{Ca}-$ und $\mathrm{Sr} / \mathrm{Ca}-W e r t e ~(4.1$, S. 78f) und hat dieses Konzentrationsmuster einen systematischen Charakter? (4.1, S. 80f)

\section{Fragestellung zum Versuchsteil 2.2:}

- Sind Unterschiede der $\mathrm{Ba} / \mathrm{Ca}$ - und $\mathrm{Sr} / \mathrm{Ca}-W e r t e$ zwischen knochenhistologisch definierten Gruppen durch die Aufreinigungsmethode bedingte Artefakte bzw. welche Auswirkungen haben modifizierte Aufreinigungsvarianten auf die Konzentrationsverhältnisse? (4.2)

\section{Fragestellung zum Versuchsteil 2.3:}

- Welche Unterschiede ergeben sich für verschiedene Variablen zur Charakterisierung diagenetisch veränderter Knochensubstanz zwischen den histologisch definierten Gruppen innerhalb verschiedener Populationen? (4.3)

\section{Fragestellung zu 2.4:}

- Gibt es innerhalb eines Kompaktaquerschnitts einer Knochenprobe vom probenintern variierenden histologischen Erhaltungszustand abhängige charakteristische Elementverteilungen? (4.4)

\section{Fragestellung zu 2.5:}

- Gibt es eine physiologische Variabilität von Spurenelementkonzentrationen innerhalb eines Femurs und hat diese einen systematischen Charakter? (4.5) 


\section{$3 \quad$ Material und Methoden}

\subsection{Material}

\subsubsection{Skelettserien für die Untersuchungen zur populationsinternen Variabilität von Spurenelementkonzentrationen}

Für diesen ersten Untersuchungsteil wurde auf bereits vorhandene Daten früherer Spurenelementuntersuchungen zurückgegriffen.

Aus den in den vergangen acht Jahren in der Historischen Anthropologie und Humanökologie Göttingen untersuchten Skelettserien (vgl. Schutkowski 1995; Schutkowski \& Herrmann 1996; Schutkowski et al. 1999; Fabig et al. 2000) wurden die Gräberfelder von Kirchheim unter Teck, Wenigumstadt und Weingarten für Untersuchungen zur populationsinternen Variabilität von Elementkonzentrationen herangezogen. Alle drei Serien verfügen über eine hinreichend große Anzahl von Individuen mit zugehörigen Datensätzen. Für die erforderlichen populationsinternen Auswertungen war hierdurch jeweils eine umfangreiche statistische Datenbasis gewährleistet. Damit waren die Voraussetzungen gegeben, einen erfolgversprechenden Vergleich zwischen diesen Populationen mit der angestrebten Musteridentifikation durchzuführen. Möglichst große primäre Stichprobenumfänge waren notwendig, da für die Fragestellung der Untersuchung eine Beschränkung der Auswertungen auf bestimmte Individuen erforderlich waren. Es wurden nur Erwachsene berücksichtigt, von welchen für die detaillierten Statistiken alle Datensätze von Knochenproben mit mittlerem histologischen Erhaltungszustand ausgeschlossen wurden (zur Begründung vgl. 2.1, S. 12ff).

Entsprechend der ausschließlichen Orientierung dieser Arbeit auf den Diagenesekontext beschränken sich die folgenden Informationen zur Archäologie und Anthropologie auf Angaben zur geographischen Lage, Zeitstellung und Individuenzahl. Kulturhistorische Aspekte finden nur für Weingarten Erwähnung, soweit sie für den Vergleich zwischen den beiden Erklärungsansätzen für Spurenelementsignaturen über Sozialgruppen oder Diagenesegruppen erforderlich sind (vgl. 2.1, S. 19ff).

Die drei Skelettserien stammen von alamannischen Friedhöfen im süddeutschen Raum. Der Zeitrahmen der Belegungen vom 4. bis $8 \mathrm{Jh}$. umfaßt die gesamte Völkerwanderungszeit (Ende des 4. Jh. bis Anfang 7. Jh.) und schließt die Merowinger-Zeit (6. Jh. bis Mitte 8. Jh.) mit ein bzw. reicht bis in das frühe Mittelalter (zur zeitlichen Einordnung vgl. Filip 1969; Überblick zur Archäologie der Alamannen in Christlein 1991). 


\section{Weingarten}

Weingarten, Kr. Ravensburg, liegt im Bodenseevorland. Die etwa 900 Gräber dieses Bestattungsplatzes datieren von der zweiten Hälfte des 5. Jh. bis Mitte des 8. Jh.

Von den 801 geborgenen Gräbern (vgl. Roth \& Theune 1995) konnte für 235 Individuen auf Spurenelementdaten zurückgegriffen werden. Die Gruppe der Erwachsenen umfaßt 193 Individuen (89 §ぇ und 104 우). Die Prämissen zur histologischen Erhaltung reduzierten diesen Datensatz auf 178 Individuen (78 ふぇ઼ und 100 우).

Für die Gegenüberstellung sozialer vs. diagenetischer Faktoren populationsinterner Elementkonzentrationsmuster ist die Sozialstratifizierung der durch die Bestatteten charakterisierten Bevölkerung relevant. Anhand der archäologischen Auswertung der Grabbeigabensituation wurden die 193 Erwachsenen in 104 Individuen niederen sowie 89 Individuen hohen sozialen Ranges eingeteilt (vgl. Roth \& Theune 1995). Für die auswertungsrelevanten 178 Individuen verbleiben 95 Rangniedere und 83 Ranghohe. Die Probenliste zu Weingarten ist im Anhang (Tabelle 25) enthalten.

\section{Kirchheim unter Teck}

Kirchheim unter Teck, Lkr. Esslingen, ist am Nordrand der schwäbischen Alb in einem Tal gelegen. Die Belegungszeit für diesen Friedhof reicht von der zweiten Hälfte des 5. Jh. bis Ende des 6. Jh.. Aus der Ergrabung von 300 der wahrscheinlich 1000 Bestattungen resultierten 167 erhaltene Funde (zur Archäologie und Anthropologie vgl. Knott 1987; Becker 1985). Von diesen Skelettfunden wurden ursprünglich 151 Individuen elementanalytisch untersucht (vgl. Schutkowski 1995). In die Auswertungen der jetzigen Arbeit sind primär die 121 Erwachsenen (64 ふふふ, 56 우, 1 unbestimmt) einbezogen worden. Für die detaillierten statistischen Analysen verblieben nach dem Ausschluß von Proben mit mittlerer histologischer Erhaltung der Knochenbinnenstruktur die Knochenprobendaten von 101

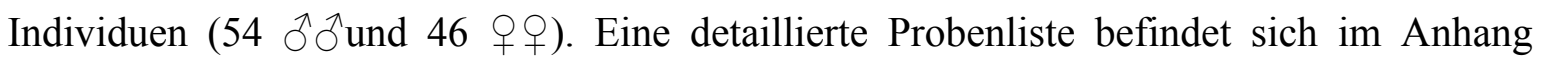
(Tabelle 26).

\section{Wenigumstadt}

Wenigumstadt, Lkr. Aschaffenburg, befindet sich im nördlichen Odenwaldvorland. Der Belegungszeitraum dieses Friedhofs umfaßt das 4. bis 8. Jh., wobei die Besiedlungsge- 
schichte sehr diskontinuierlich erscheint. Entsprechend zeigt auch die Bevölkerungszusammensetzung hinsichtlich Herkunft und Wohlstand eine heterogene Charakteristik (zur Archäologie vgl. Stauch 2000).

Für diese Serie standen Daten von 114 Individuen der 304 geborgenen Bestattungen zur

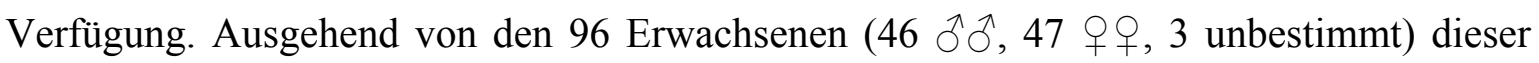
Stichprobe konnten dem histologischen Erhaltungszustand entsprechend 86 Individuen (42 $\widehat{\jmath}, 42$ 우, 2 unbestimmt) in den Auswertungen berücksichtigt werden. Die Probenliste kann im Anhang (Tabelle 27) eingesehen werden.

\subsubsection{Material für die Untersuchungen zur Probenaufreinigung und für die mikroanalytischen Untersuchungen}

Für den Versuch zur Probenaufreinigung sowie die Einzelprobe für die mikroanalytischen Untersuchungen wurden Knochenproben eines bisher elementanalytisch unbearbeiteten Skelettkollektivs genutzt.

\section{Kirchheim am Ries}

Die verwendete Skelettserie ist ebenfalls alamannischen Ursprungs. Es handelt sich um Knochenfunde von einem Reihengräberfriedhof in Kirchheim am Ries, Ostalbkreis. In der zweiten Hälfte des 6. Jh. beginnend, erfolgten hier Bestattungen bis Anfang des 8. Jh. Die Belegungszeit ordnet diesen Friedhof somit in die Zeit der Merowinger ein (zur Archäologie vgl. Neuffer-Müller 1983, zur Anthropologie zusätzlich mündl. Mitteilungen Czarnetzki). Von den mehr als 500 ergrabenen Bestattungen wurden Knochenproben von 94 erwachsenen Individuen (48 $\widehat{\partial} \widehat{\delta}$ und 46 우) entsprechend den limitierenden Vorgaben der Versuchsplanung entnommen. Diese erforderte eine Beschränkung auf Femurproben von Männern im Alter von 20-50 Jahren und Frauen im Alter von 20-40 Jahren (vgl. 2.2). Nach Auswertung der Knochenhistologien (Ausschluß von Proben mit mittlerer Erhaltung

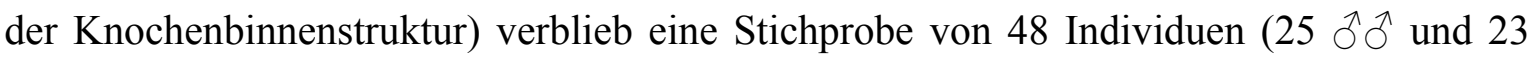
웅). Eine entsprechende Auflistung befindet sich im Anhang (Tabelle 28).

Ein wichtiger Aspekt für diesen Untersuchungsteil ist die Lage der untersuchten Individuen im Gräberfeld. Bei der Probenauswahl wurde gezielt sichergestellt, daß die Verteilung der histologisch gut erhaltenen und der histologisch schlecht erhalten untersuchten Proben über das gesamte Gräberfeld zufällig ist. Hierdurch wurde ein mögliche räumliche und 
damit bodenchemische Gruppenassoziation der Proben vermieden (vgl. 2.2). In Abbildung 4. ist die Verteilung der Proben über das Gräberfeld dargestellt.

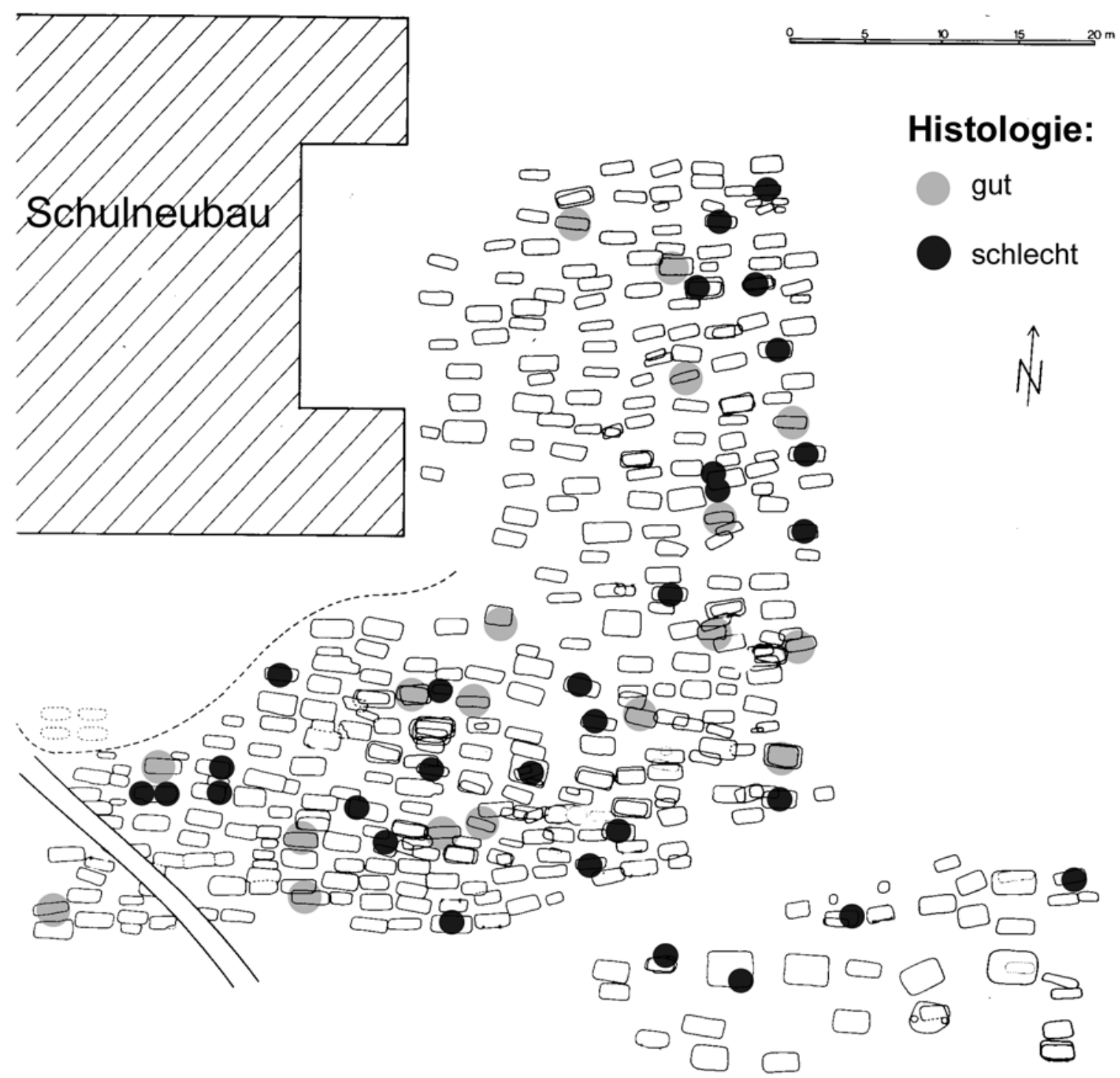

Abbildung 4: Lageplan der Bestattungen des Gräberfeldes von Kirchheim am Ries mit markierten Grabstellen der untersuchten Knochenproben kategorisiert nach histologischem Erhaltungszustand. 
Einzelprobe von Kirchheim am Ries für die mikroanalytischen Untersuchungen

Für die Untersuchungen zu den Elementverteilungen und -konzentrationen in einem Kompaktaquerschnitt wurde eine jener Knochenproben von Kirchheim am Ries genutzt, die infolge ihres mittleren Erhaltungszustandes der Knochenbinnenstruktur von den Untersuchungen zur Probenaufreinigung ausgeschlossen wurden (Probenbezeichnung KR 261). Durch die Verwendung einer solchen Probe mit einer Mischung aus gut und schlecht erhaltenen Arealen der Knochenbinnenstruktur wurde der direkte Vergleich der damit einhergehenden wechselnden Mineralcharakteristika ermöglicht.

\subsubsection{Rezentes Knochenmaterial für die Untersuchung zur intraossären Variabilität von Elementkonzentrationen}

Zur Erfassung knocheninterner Unterschiede von Elementkonzentrationen konnte Sektionsmaterial verwendet werden. Die begrenzte Anzahl von Sektionen sowie das notwendige Einverständnis des Patienten bzw. der Angehörigen zur Freigabe von Knochenmaterial vermindert die Probenauswahl erheblich. Die altersabhängigen Veränderungen des Knochenminerals im Allgemeinen, aber insbesondere die durch die Menopause bedingten kurzfristigen hormonphysiologischen Effekte bei Frauen, bedeuten einen weiteren Probenausschluß.

Dieser Teilversuch wurde aber auch versuchslogisch als Abschätzungsexperiment auf die Analyse einer Femurdiaphyse beschränkt (vgl. 2.5). Durch die Auswahl eines männlichen Individuums sind nur kontinuierliche hormonphysiologisch bedingte Alterungsprozesse des Knochenminerals von Relevanz. Das Alter von 67 Jahren des untersuchten Mannes kann mit dieser Begründung als unkritisch bewertet werden, auch wenn diese Analysen zur intraossären Variabilität als eine Kontrolle für die Untersuchungen zur Probenaufreinigung mit deren Altersbeschränkung für Männer auf 20 bis 50 Jahre dienen sollte (vgl. 2.2). Die Anamnese des Patienten, einschließlich der zum Tode führenden Krankheit, enthält keine knochenmineralphysiologisch langfristig relevanten Erkrankungen. Auch die Medikation, soweit bekannt, wird als diesbezüglich unbedenklich betrachtet. Das zum Tode führende maligne Geschehen war durch einen rapiden Verlauf gekennzeichnet. Von der operativen Entfernung des Primärtumors bis zum Tode vergingen weniger als vier Monate. Die auf die Operation folgende Bestrahlung war lokal auf das Operationsgebiet und die Wirbelknochen beschränkt und erfolgte ohne zusätzliche Chemotherapie. Da mineralische Umsetzungen im Knochen ein langfristiger, mehrjähriger Prozeß sind, kann eine generelle 
Beeinflussung der Knochenmineralmatrix und insbesondere eine des von der Erkrankung nicht betroffenen Femurs angenommen werden.

\subsection{Methoden}

\subsubsection{Probenvorbereitung}

Die folgendenden Arbeitsschritte der Spurenelementanalytik an bodengelagertem Skelettmaterial (vgl. Schutkowski 1994) sind teilweise für die verschiedenen Teiluntersuchungen allgemein gültig. Auf diese Angaben wird in den weiteren Methodenkapiteln daher Bezug genommen werden und nur bei Modifikationen bzw. Optimierungen erfolgt eine ausführlichere Erläuterung.

Vorangestellt wird Grundsätzliches zum kontaminationsvermeidenden spurenanalytischen Arbeiten:

Aus der Kontaminationsgefahr, die jeder Kontakt mit dem Probenmaterial während der einzelnen Arbeitsschritte sowie die Aufbewahrung der Proben in jeglichen Gefäßen bedeutet, ergeben sich Anforderungen an die spurenanalytische Reinheit der verwendeten Gegenstände.

Eine Säurebehandlung in Form von Ausdämpfen mit Salpetersäure (65\%) für alle verwendeten Glasgefäße und Gegenstände bzw. eine ultraschallunterstützte Behandlung mit warmer 2\%-iger Salpetersäure für die Kunststoffmaterialien ging daher deren Benutzung grundsätzlich voraus. Voll- und Meßpipetten sowie Pipettenspitzen für die verschiedenen Eppendorf ${ }^{\mathbb{B}}$-Pipettierhilfen sind ebenfalls mit 2\%-iger Salpetersäure gereinigt worden, ebenso jegliche Aufbewahrungsgefäße bzw. Vorratsbehälter (PP-, LDPE- bzw. FEPGefäße) für Stammlösungen und Verdünnungsmedien. Den Abschluß der Reinigungsprozedur bildete für alle Materialien das wiederholte Spülen mit Aqua bidest. und die anschließende Trocknung bei $50^{\circ} \mathrm{C}$.

Kunststoffpinzetten, teflonisierte Stahlspatel und Glasspatel wurden ebenfalls jeweils vor Gebrauch und bei Probenwechsel durch Tauchen in 2\%-ige Salpetersäure und wenn erforderlich (Spatel) durch Abreiben mit einem Präzisionswischtuch (Kimwipes ${ }^{\circledR}$ ) gereinigt. Durch anschließendes Tauchen in Ethanol bzw. Aceton (entsprechend der Chemikalienbeständigkeit) wurden die Gegenstände beschleunigt getrocknet. Der Achatmörser einschließlich Pistill für die Probenhomogenisierung wurde bei Probenwechsel dreimal mit 
reichlich Aqua bidest. gespült und nach jedem Spülvorgang zusätzlich mit einem neuen Präzisionswischtuch gesäubert sowie abschließend mit einem ethanolgetränkten Präzisionswischtuch trockengerieben.

Nichtverschließbare Glaswaren oder Kunststoffgefäße waren bei Nichtgebrauch grundsätzlich mit Parafilm $\mathrm{M}^{\circledR}$ verschlossen.

Die Arbeiten an den Proben erfolgten mit puderfreien Latex-Untersuchungshandschuhen (Hartmann oder Safeskin ${ }^{\circledR}$ ) und nach der Probenaufreinigung ausschließlich mit gereinigten Plastikpinzetten (s.o.).

Die Herstellung der Stammlösungen bzw. alle weiteren Arbeiten zur Meßlösungsvorbereitung erfolgten unter einem Laminar-Air-Flow-System (Schymura Maschinen-Gerätebau).

\subsubsection{Entnahme der Knochenproben}

Notwendige Voraussetzung für die Beprobung eines Individuums war die makroskopisch als hinreichend bewertete Erhaltung der Knochensubstanz. Unabhängig davon bedeuteten abiogene, wahrscheinlich metallische Verfärbungen durch Grabbeigaben, den Ausschluß des entsprechenden Skelettelements von der Beprobung.

Die Probenentnahme erfolgte mit einer Diamanttrennscheibe (Horico) unter Verwendung eines Dentalmikromotors (K-10-Handstück mit zugehörigem Steuergerät von KaVoEWL). Die Knochenstücke wurden den Empfehlungen aus der Literatur (Grupe 1988) entsprechend standardisiert aus der Mitte einer Femurdiaphyse anterior entnommen. Für die früheren Untersuchungen zur populationsinternen Variabilität von Spurenelementkonzentrationen waren Analysen von einer möglichst großen Individuenzahl erforderlich. Unter Annahme einer relativ geringen intraindividuellen Variabilität von Spurenelementkonzentrationen ist bei Fehlen oder mangelnder Erhaltung der Femora auch auf andere Skelettelemente zurückgegriffen worden. Für eine Beprobung wurden dann Humerus und Tibia präferiert.

Die Größe der entnommenen Probenstücke von ca. $1 \times 2 \mathrm{~cm}$ (transversal $\times$ longitudinal) entspricht einer Knochenmasse von 1-3 g. Für den Versuchsteil zur Überprüfung der Probenaufreinigung waren für die mehrfachen Analysen größere Probenstücke von ca. $1 \times 4 \mathrm{~cm}$ mit einer durchschnittlichen Masse von 2-6 g erforderlich. 


\subsubsection{Herstellung der histologischen Knochenpräparate}

Für die histologischen Präparate wurden von den entnommenen Knochenstücken durch einen transversalen Schnitt Teilproben abgetrennt. Diese wurden jeweils einzeln in einem Kunststoffgefäß (hierfür haben sich Filmdosen als geeignet erwiesen) in Biodur ${ }^{\mathrm{TM}}$ [Biodur Products, Epoxidharz E12 (100 Gewichtsanteile) und Härter E1 (28 Gewichtsanteile)] eingebettet. Unter Verwendung eines Exikkators mit Wasserstrahlpumpe wurden die Proben-Biodur-Ansätze eine Stunde entgast und anschließend über drei Tage bei RT ausgehärtet.

Die ausgehärteten Proben-Biodur-Blöcke wurden mit einer Schneidmaschine (Bodo Schmidt, Göttingen) für die Verwendung einer Innenlochsäge (Leitz $\left.{ }^{\circledR} 1600\right)$ zugeschnitten. Mit dieser erfolgte anschließend die Anfertigung der Dünnschnitte (Transversalebene). Die Schnittdicke wurde in Abhängigkeit von der Stabilität des Knochenmaterials zwischen 60 und $100 \mu \mathrm{m}$ variiert.

Für die mikroskopische Begutachtung der Knochenbinnenstruktur sind diese Dünnschnitte mit Eukitt und einem Deckgläschen auf einem Objektträger fixiert worden.

Für die Teiluntersuchung zum Interpopulationsvergleich der drei Serien Kirchheim unter Teck, Wenigumstadt und Weingarten waren diese histologischen Präparate bereits vorhanden. Für die Proben von Kirchheim am Ries wurden sie entsprechend der geschilderten Vorgehensweise neu angefertigt.

\subsubsection{Probenaufreinigung}

Die folgenden Erläuterungen zur Aufreinigung der Knochenproben beziehen sich auf die laborintern standardisierte Vorgehensweise (vgl. Schutkowski 1994). Diese war für die Durchführung der Analysen für die drei Skelettserien des ersten Untersuchungskomplexes dieser Arbeit (vgl. 2.1) maßgeblich. Gleichzeitig bildet sie aber die Grundlage bzw. den Ausgangspunkt für die Untersuchungen zur Probenaufreinigung.

\section{Vorreinigung}

Von den entnommenen Knochenkompaktastücken wurden zunächst alle Spongiosareste mit einem Skalpell abgelöst. Hierauf folgte eine grobe Entfernung aller den Knochenstü- 
cken anhaftenden Sedimentreste, sowie eine weitere Reinigung unter fließendem Leitungswasser mit einer Zahnbürste.

\section{Ethern}

Zur Entfernung etherlöslicher organischer Substanzen wurden die Knochenproben einer vierstündigen Etherextraktion mit Diethylether (reinst) in einem Soxhlet-System unterzogen. Durch dieses Herauslösen fettartiger unpolarer Substanzen werden durch sie bedingte mögliche Trenneffekte minimiert. Dadurch sind Knochenmineral und mögliche anorganische Fremdsubstanz sowohl an der Oberfläche als auch in Hohlräumen des Knochens einem wässrigen Medium verbessert zugänglich. Für den anschließenden entscheidenden Arbeitsschritt zur Probenaufreinigung, dem Ätzen mit einer organischen Säure als wässriger Lösung, werden hiermit optimierte Voraussetzungen geschaffen. Insbesondere werden durch die Etherextraktion auch mögliche Reaktionsinterferenzen zwischen organischen Substanzen und der Säure minimiert.

Ätzen

Grundlage für diesen Arbeitsschritt bilden die theoretischen Überlegungen, daß innerhalb einer Knochenprobe diagenetisch verändertes Mineral gegenüber nativem Knochenmineral eine erhöhte Löslichkeit aufweist (vgl. 1.2, S. 7).

Das Ätzen mit einer Säure bedeutet eine Behandlung der Knochenproben in einer wässrigen Lösung mit stark abgesenktem pH-Wert. Hieraus folgt eine generell erhöhte Löslichkeit für alle mineralischen Substanzen. Diagenetische Mineralanteile, Rekristallisationsprodukte, aber auch natives Knochenmineral gehen entsprechend ihrer abgestuften Löslichkeit in unterschiedlichem Maße in Lösung. Bei einer hinreichenden Säurebehandlung, einschließlich des vollständigen Abtransports des gelösten Materials, sollten alle knochenfremden Mineralkomponenten sowie oberflächennahe native Knochenmineralanteile aus der Probe entfernt worden sein. Hierbei ist "oberflächennah" im erweiterten Sinne dahingehend zu verstehen, daß auch Mineraloberflächen in Knochenhohlräumen gemeint sind.

Dieser Annahme folgend wurden die Knochenproben mit ca. $20 \mathrm{ml}$ Ameisensäure (98-100\%) in einem Rollrandschnappdeckelglas, unterstützt durch Ultraschall (Ultra- 
schallbad Sonorex RK 102), geätzt. Diesem eigentlichen Ätzvorgang von 2-5 Minuten (in Abhängigkeit von der Kompaktastärke) folgten zehn Spülgänge in Aqua bidest. (jeweils ca. $20 \mathrm{ml}$ ), ebenfalls unter Ultraschalleinwirkung ${ }^{2}$.

\subsubsection{Vorbereitung der Proben zur Elementanalytik in Lösung}

Sowohl die Konzentrationsbestimmung mit AAS als auch die gewählte Variante der ICP-OES-Analytik erfordert die Überführung der Probensubstanz in Lösung, in welcher sie den Atomisierungseinrichtungen zugeführt wird (vgl. 3.2.2.1, S. 52). Hierfür sind zwei weitere Arbeitsschritte erforderlich.

\section{Veraschung}

Die Reduktion des Knochenmaterials auf seine mineralischen Komponenten erfolgte durch die Veraschung der Probenstücke in einem Muffelofen (Heraeus ${ }^{\circledR}$ KM 260) bei $500{ }^{\circ} \mathrm{C}$ über $12 \mathrm{~h}$. Diese laborintern standardisierten Veraschungsparameter (vgl. Schutkowski 1994) bilden ein Optimum für die gewünschten Veränderungen der Knochensubstanz bei gleichzeitig minimierten konzentrationsverändernden Effekten durch flüchtige Elementverbindungen (vgl. Edward et al. 1990). Der Veraschungsprozeß bedeutet eine Anreicherung der $\mathrm{zu}$ bestimmenden Elemente pro Masseeinheit Probensubstanz, dient darüber hinaus aber der Verbesserung der Löslichkeit des Materials im anschließenden Aufschluß.

\footnotetext{
${ }^{2}$ An dieser Stelle ist ein Hinweis bezüglich eines der bisher präferierten Prüfkriterien für diesen Aufreinigungsschritt erforderlich:

Die mit der Anzahl der Spülvorgänge fortschreitende Verdünnung der Ameisensäure resultiert in einem steigenden $\mathrm{pH}$-Wert der Lösungen. Während der wiederholten Spülvorgänge sollten daher in zunehmendem Maße nur noch leichter lösliche bzw. auch bei höheren pH-Werten lösliche mineralische Komponenten aus der Knochenprobe in Lösung gehen. Dies bildete die Grundlage dafür, die Elementkonzentrationen in den Spüllösungen als Referenz für den Reinigungsprozeß heranzuziehen.

Hierzu wurden jeweils die Lösungen (mit definierten Volumina) aller Spülgänge einer Knochenprobe hinsichtlich ihrer Elementgehalte analysiert. Minimierte Konzentrationen in den letzten Spüllösungen gegenüber den vorangegangenen wurden als Beleg für einen Aufreinigungserfolg herangezogen. Atypisch erhöhte Elementkonzentrationen wären als Indiz gewertet worden, daß noch relevante Anteile leichter löslicher (diagenetisch veränderter) Mineralbestandteile vorhanden sind (vgl. z.B. Schutkowski et al. 1999).

Erwartungsgemäß niedrige Konzentrationen ermöglichen aber letztlich keine qualitative Aussage zum Aufreinigungserfolg, da ihr Informationsgehalt lediglich auf die Feststellung beschränkt ist, daß bei nahezu säurefreien Lösungen (mit annähernd neutralem pH-Wert) nur noch geringe Mengen Mineral in Lösung gehen. Erst ein Vergleich mit einer entsprechenden Lösung von einem Knochen vor dessen Säurebehandlung hätte Aufschluß darüber geben können, inwieweit leichter lösliche Bestandteile der Knochenprobe durch das Ätzen quantitativ herausgelöst wurden.

Die Ergebnisse der Analysen der Spüllösungen werden daher in die Auswertungen der vorliegenden Untersuchung nicht einbezogen.
} 
Außerdem ist aber eine annähernde Bestimmung des organischen Masseanteils der Knochen möglich (vgl. 2.3, S. 32f).

\section{Herstellung der Stammlösungen}

Nach der Veraschung erfolgte die Homogenisierung der Knochenproben in einem Achatmörser.

Den Abschluß der Probenvorbereitung bildete der Säuredruckaufschluß von jeweils ca. 70 mg Knochenpulver mit $1 \mathrm{ml}$ Salpetersäure (65 \%, Suprapur $\left.{ }^{\circledR}\right)$ in Quarzgläsern innerhalb einer Druckaufschlußapparatur (Seif-Aufschlußtechnik) bei $160^{\circ} \mathrm{C}$ in einem Trockenschrank (Heraeus ${ }^{\circledR}$ ) über $6 \mathrm{~h}$.

Aus den einzelnen Aufschlußlösungen wurden durch zehnfache Verdünnung (Zugabe von $9 \mathrm{ml}$ Aqua bidest. zu $1 \mathrm{ml}$ Säureaufschluß) die Stammlösungen $(10 \mathrm{ml})$ für die Analysen hergestellt und bei $-20{ }^{\circ} \mathrm{C}$ in LDPE-Gefäßen bis zur Messung gelagert.

\subsubsection{Massekontrollen während Probenaufreinigung und -vorbereitung}

Für Teilaspekte der Fragestellung sind die charakteristischen Masseverluste während der Probenvorbereitungsschritte Ätzen und Veraschen von Bedeutung (vgl. 2.3, S. $31 \mathrm{ff}$ ).

Die in allen früheren Untersuchungen routinemäßig durchgeführten Masseüberprüfungen während der Arbeitsschritte Ätzen und Veraschen bilden hierfür die erforderliche Datengrundlage. Zur Wägung sind die Probenstücke vor bzw. nach dem jeweiligen Arbeitsschritt im Trockenschrank bei $50^{\circ} \mathrm{C}$ bis zur Gewichtskonstanz getrocknet worden. Alle Wägungen erfolgten mit einer Halbanalysenwaage (Sartorius ${ }^{\circledR}$ R 160 P).

\subsubsection{Modifikationen der Probenvorbereitung für den Untersuchungsteil zur Probenaufreinigung}

An dieser Stelle werden nur jene beiden Probenvorbereitungsschritte für diesen Versuchsteil erörtert, die von den allgemeinen in den vorangegangen Kapiteln dargelegten abweichen. Die Vorbereitung der Proben in diesem Versuch ist also hinsichtlich der anderen Arbeitschritte die hier nicht erwähnt werden [Probenentnahme; Histologieherstellung; Probenaufreinigung (ausgenommen Ätzen); Vorbereitung zur Analytik, Massekontrollen], diesen identisch gewesen. 


\section{Mechanische Oberflächenbehandlung}

Entsprechend des Versuchsdesigns zur Überprüfung der Probenaufreinigung wurde bei zwei der vier Teilprobensätze von Kirchheim am Ries als Probenaufreinigungsschritt eine mechanische Oberflächenbehandlung der Knochenproben durchgeführt (vgl. 2.2). Dieser Arbeitsschritt war der chemischen Aufreinigung (Ethern und Ätzen) vorangestellt. Mit Hilfe eines Ceramic-Messers (Heinr. Böker Baumwerk) wurde von den Knochenproben sowohl periost- als auch markraumseitig die Oberflächenschicht abgeschabt.

Von der entfernten Knochensubstanz wurden durch Vermessung (minimale und maximale Probendicke) und Wägung der Knochenproben vor und nach der Oberflächenbehandlung als Kontrolldaten die Schichtdicken (Differenz der Probenstärken in mm) und die Massen (Massendifferenz bezogen auf die Ausgangsmasse in \%) dokumentiert. Diese Datenerfassung erfolgte separat für die Periostseite (im Mittel 0,8 mm bzw. 17\%) und die Markraumseite (im Mittel 1,6 mm bzw. 20\%). Da Sedimentkontakt und -wechselwirkung bis in größere Tiefen der Knochenstruktur durch größere Hohlräume in der markraumseitig spongiöseren Kompaktastruktur begünstig waren, wurde dort die Oberfläche verstärkt entfernt. Hieraus resultieren für die Markraumseite im Vergleich zur Periostseite die deutlich größeren Schichtdicken für die entfernte Knochensubstanz bei gleichzeitig nur geringfügig größeren Massen derselben (s.o.).

Diese einzelprobenbezogenen Kontrolldaten der Oberflächenbehandlung wurden für eine möglicherweise erforderliche Überprüfung der durch diese Probenvorbereitung bedingten Konzentrationsänderungen in den Knochenproben erfaßt. Bei relevanten Konzentrationsänderungen sind damit Abhängigkeiten zwischen diesen und dem Grad der Oberflächenbehandlung überprüfbar (vgl. 2.2).

\section{Optimiertes Ätzen}

Im Rahmen des Aufreinigungsversuchs wurden zwei Probensätze von Kirchheim am Ries geätzt (vgl. 2.2). Die Vorgehensweise glich dabei prinzipiell der in 3.2.1.3 (S. 46f) beschriebenen Ätzmethode. Zur Optimierung des Lösungsprozesses wurden jedoch beim Ätzen Modifikationen vorgenommen.

Für verbesserte Lösungsbedingungen bereits während des Ätzvorganges wurde der Wasseranteil der Säurelösung erhöht. Hierfür wurde die Ameisensäure (98-100\%) 1:2,5 verdünnt (resultierender Säuregehalt: 40\%). Der Ätzvorgang wurde standardisiert und damit 
unabhängig von der Kompaktastärke der jeweiligen Probe mit 5 min Dauer unter Ultraschalleinwirkung durchgeführt. Auf diesen Ätzvorgang folgten fünf Spülgänge in Aqua bidest. Ein solcher Ätzzyklus wurde für jede Probe viermal wiederholt, wobei der letzte Zyklus durch zehn statt der fünf Spülgänge mit Aqua bidest. abgeschlossen wurde.

Durch diese Prozedur des wiederholten Ätzens mit alternierender Wasserspülung wird den für Lösungsprozesse von Apatiten und apatitähnlichen Ca-P-Verbindungen spezifischen Reformations- und Repräzipitationsprozessen an den Mineraloberflächen Rechnung getragen. Der Minerallösungsprozeß wird hierdurch effektiviert (zur spezifischen Charakteristik der Löslichkeit bzw. von Lösungsprozessen von Apatiten und anderen knochenimmanten Mineralen vgl. Baig et al. 1999a; Baig et al. 1999b; Chhettry et al. 1999; Shellis et al. 1999; Dorozhkin 1997).

\subsubsection{Probenvorbereitung für Mikroanalysen}

Für die beiden mikroanalytischen Verfahren (EPMA und LA-ICP-TOFMS; vgl. 3.2.2.2) wurde die hierfür ausgewählte Knochenprobe (KR 261; vgl. 2.1, Abbildung 2) gemeinsam genutzt. Hierfür ist von dieser Probe zunächst mit einer Diamanttrennscheibe transversal ein ca. $5 \mathrm{~mm}$ starkes Teilstück abgetrennt worden. Nach Entfernen des Sägestaubes mit Zahnbürste und Aqua bidest. wurde dieses Probensegment bis zur Gewichtskonstanz getrocknet.

Den Erfordernissen für die EPMA entsprechend erfolgte anschließend die Einbettung der Probe in einem elektronenstrahlstabilen Medium auf silikatischer Basis (neu entwickeltes laborintern standardisiertes Verfahren von A. Kronz, Abteilung Geochemie des Göttinger Zentrums Geowissenschaften; Publ. in Vorb.). Die für die Analyse vorgesehene transversale Fläche der Knochenprobe (ein vom Periost bis zum Markraum reichender Querschnittsektor der Kompakta) wurde plan geschliffen und mit einer Endfeinheit von $1 \mu \mathrm{m}$ (Korngröße des Diamantschleifmittels) poliert. Zur Ableitung der elektronenstrahlbedingten Aufladungen wurde die Oberfläche mit Kohlenstoff bedampft (Schichtdicke ca. $150 \AA$ mit Widerstandsbedampfung; spektralreine Kohleelektrode). 


\subsubsection{Vorbereitung des rezenten Knochenmaterials}

Für die Bestimmung der intraossären Variabilität der Elementkonzentrationen wurde die Diaphyse eines rezenten Femurs untersucht. Entsprechend den experimentellen Vorgaben in 2.5 wurde hierfür aus dem Gesamtknochen zunächst ein Abschnitt von $10 \mathrm{~cm}$ (von $5 \mathrm{~cm}$ proximal bis $5 \mathrm{~cm}$ distal der Diaphysenmitte) mit einer Bandsäge (Black \& Decker ${ }^{\circledR}$ ) herausgesägt und in eine mediale bzw. laterale Hälfte getrennt. Der Bereich der Linea aspera wurde hiernach bei beiden Diaphysenhälften als schmaler Knochenstreifen abgetrennt. Anschließend sind noch anhaftende Weichgewebereste sowie das Knochenmark einschließlich des größten Teils der Spongiosa entfernt worden.

Die dann folgenden Schritte der Probenaufteilung erfolgten mit einer Diamanttrennscheibe (vgl. 3.2.1.1). Beide Diaphysenhälften wurden jeweils in vier proximal-distale Streifen von ca. $1 \mathrm{~cm}$ (periostseitige Breite) geteilt. Von den erhaltenen Knochenstreifen wurden die nach dem ersten Abschaben noch verbliebenen Spongiosa- und Weichteilreste entfernt. Anschließend wurde jeder der Streifen in fünf Stücke von ca. $2 \mathrm{~cm}$ Länge gesägt. Es resultieren hiernach für fünf Diaphysenebenen je acht Probenstücke ( $2 \times$ anterior, $2 \times$ medial, $2 \times$ lateral und posterior je eine Teilprobe medial und lateral der Linea aspera; Einzelprobenmasse 1-3 g). Horizontale und longitudinale Lokalisationen der Einzelproben wurden dokumentiert. Die Proben wurden nach Abschluß der Sägearbeiten mit einer Zahnbürste und Aqua bidest. von anhaftendem Sägestaub befreit.

Die weitere Aufreinigung dieser rezenten Knochenproben beschränkte sich auf die Etherextraktion analog 3.2.1.3 (S. 46). Die abschließende Vorbereitung zur Elementanalytik (Veraschung und Herstellung der Stammlösungen) erfolgte wie in 3.2.1.4 erläutert. 


\subsubsection{Analytische Methoden}

\subsubsection{Konzentrationsbestimmungen mit AAS, Photometrie und ICP-OES} AAS-Analytik

Die Konzentrationsbestimmungen für die Knochenproben der drei Skelettserien Kirchheim unter Teck, Wenigumstadt und Weingarten für den Interpopulationsvergleich waren bereits vor Beginn und unabhängig von dieser Arbeit im Rahmen vorangegangener Forschungsvorhaben abgeschlossen. Die Informationen zu dieser Analytik werden aber der Vollständigkeit und Vergleichbarkeit wegen mit aufgeführt.

Die Analysen der Elemente $\mathrm{Ca}, \mathrm{Sr}$ und $\mathrm{Ba}$ in den hergestellten Stammlösungen erfolgten unter Verwendung der Atomabsorptionsspektrometrie (AAS). Die Analysen wurden mit Flammenatomisierungstechnik durchgeführt [Flame Atomic Absorption Spectrometry (FAAS), Übersicht zu den AAS-Techniken und deren Nomenklatur in Welz 1997 und Jackson \& Mahmood 1994]. Basisgerät war das Spektrometer Perkin Elmer ${ }^{\circledR}$ AAS 1100 B. Als Lichtquellen wurden elementspezifische, d.h. wellenlängenspezifische Hohlkathodenlampen verwendet [(Einelementlampen für $\mathrm{Ba}$ und $\mathrm{Sr}$ sowie eine Zweielementlampe für $\mathrm{Ca}$ (für $\mathrm{Zn}$ als zweites Element geeignet)].

Die Methodik entspricht prinzipiell den allgemeinen Vorgaben von Welz (1997) bzw. den vom Gerätehersteller (Perkin Elmer) beschriebenen Standardbedingungen für das jeweilige Element. Die geräteinternen Analyseparameter für die Bestimmung der einzelnen Elemente sind im Anhang (Tabelle 11) aufgelistet. Die Kalibrierung des Signalausgabesystems des AAS-Gerätes erfolgte mit wässrigen Standardlösungen definierter Konzentration, die durch entsprechende Verdünnung zertifizierter Standardlösungen (Merck) hergestellt wurden.

Als Meßlösungen wurden in Abhängigkeit von den Elementkonzentrationen sowie den Meßbereichen für den jeweiligen Analyten variable Verdünnungen von Teilvolumina der Probenstammlösungen hergestellt. Die Konzentrationen der Meßlösungen wurden unter den im Anhang (Tabelle 12) aufgeführten Meßbedingungen (Kalibrierung, Verdünnungsraten und -medien) bestimmt. Die Umrechnung dieser Meßlösungskonzentrationen auf die Elementgehalte in der Knochenprobe wird in 3.2.3.1 zusammen mit den entsprechenden Angaben zu den anderen Analysemethoden erläutert. 


\section{Photometrische Bestimmung des Phosphorgehaltes}

Da die Absorptionsmaxima bzw. die elementspezifischen Wellenlängen für Phosphor im Vakuum-UV-Bereich liegen, war eine Bestimmung mit der für diese früheren Untersuchungen verwendeten AAS-Technik nicht möglich (vgl.: Welz 1997, S. 571).

Die Bestimmung des Phosphorgehaltes erfolgte daher über die Extinktionsbestimmung der aus Phosphor und Natriummolybdat gebildeten Molybdatophosphorsäure. Zur Konzentrationsbestimmung wurden unter Verwendung einer Molybdatlösung und 2,5 $\mathrm{N} \mathrm{HNO}_{3}$ entsprechende Meßlösungen angesetzt. Diese entsprachen einer Probenverdünnung von 1:100. Die resultierende Konzentration der Molybdatophosphorsäure ist dem Phosphorgehalt der Meßlösung proportional. Bei einer für Molybdatophosphorsäure spezifischen Wellenlänge von $\lambda=405 \mathrm{~nm}$ (Maximumflanke in $\mathrm{H}_{2} \mathrm{O}$ ) wurde die Extinktion der Lösungen photometrisch bestimmt (Photometer Eppendorf ${ }^{\circledR} 1101 \mathrm{M}$ ). Die Konzentrationsberechnung für die Knochenproben wird in 3.2.3.1 erläutert.

\section{ICP-OES-Analytik}

Für den Versuchsteil zur Überprüfung der Probenaufreinigung wurden für die Knochenproben der Skelettserie Kirchheim am Ries neben den Konzentrationen für Ca, P, Ba und Sr auch die für Al und Mg bestimmt. Zusätzlich erfolgten für eine Stichprobe Konzentrationsbestimmungen für Na. Dieses erweiterte Elementspektrum wurde ebenfalls für die Untersuchung zur intraossären Konzentrationsvariabilität in einer Femurdiaphyse analysiert.

Die Konzentrationsmessungen der vorbereiteten Stammlösungen der entsprechenden Knochenproben wurden hierfür in der Abteilung Geochemie des Göttinger Zentrums Geowissenschaften unter Verwendung der ICP-OES-Technik (Inductively Coupled Plasma Optical Emission Spectrometry) durchgeführt. Diese Technik bietet durch die simultane Multielementanalyse neben einer beschleunigten Durchführung der Analysen den entscheidenden Vorteil einer verbesserte Richtigkeit der bestimmten probeninternen Konzentrationsquotienten. Da die Analyse nach einmaliger Probenverdünnung stattfindet, werden mögliche analytische Fehler ausgeschlossen, die infolge voneinander unabhängiger Verdünnungsvorgänge für jedes einzelne Element bei der AAS-Analytik möglich sind. Hinzu kommen als prinzipielle Vorteile dieser Technik die erhöhte Atomisierungseffektivität und die daraus resultierende Verminderung chemischer Störungen sowie die ebenfalls geringen Ionisationsstörungen (Überblick zur ICP-OES-Analytik in Skoog \& Leary 1996). 
Die Analysen wurden mit einer ICP-OES OPTIMA $3300^{\mathrm{TM}}$ DV mit Autosampler AS-91 (beide Geräte Perkin Elmer ${ }^{\mathrm{TM}}$ ) durchgeführt. Aus den elementspezifischen Emissionsspektren wurden pro Element die Intensitäten mehrerer Wellenlängen (3 bzw. 4) zur Analyse genutzt (geräteseitige Parameter vgl. Anhang, Tabelle 13; elementspezifische Analysebedingungen vgl. Anhang, Tabelle 14).

Die Kalibrierung erfolgte mit wässrigen Multielementstandardlösungen definierter Konzentrationen, welche zunächst aus Multielementstammlösungen (Bernd Kraft) durch entsprechende Verdünnungen hergestellt wurden (vgl. Tabelle 15).

Für alle Analysen mit der ICP-OES-Technik wurden die Stammlösungen der Knochenproben standardisiert 1:7 verdünnt (zur Berechnung der Elementgehalte der Knochenproben vgl. 3.2.3.1).

\subsubsection{Mikroanalysen mit EPMA und LA-ICP-TOFMS}

Die Untersuchung des Kompaktaquerschnittes einer Knochenprobe (KR 261 des Gräberfeldes Kirchheim am Ries) hinsichtlich Elementverteilungen und -konzentrationen untergliedert sich in zwei Teilanalysen. Die höher konzentrierten Hauptelemente (Ca, P, Mg, Na und S) wurden mit EPMA (Electron Probe Microanalysis bzw. -analyser) analysiert. Die Messungen für die Elemente ( $\mathrm{Sr}, \mathrm{Ba}$ und $\mathrm{U}$ ) erfolgten mit der LA-ICP-TOFMS (Laser Ablation Inductively Coupled Plasma Time of Flight Mass Spectrometry).

Die Analysen mit beiden genannten Techniken wurden ebenfalls in der Abteilung Geochemie des Göttinger Zentrums Geowissenschaften durchgeführt.

\section{EPMA}

Die Analyse von Materialoberflächen mit EPMA zeichnet sich durch eine hohe Ortsauflösung im Mikrometerbereich aus. Durch die quantitative Auswertung elektronenstrahlinduzierter, elementspezifischer Röntgenstrahlung können hiermit Elementverteilungen kleinsträumiger Oberflächenstrukturen analysiert werden (zur EPMA vgl. Überblick in Skoog \& Leary 1996 bzw. detailliert in Scott et al. 1995).

Die hohe Auflösung ist aber auch mit erhöhten Nachweisgrenzen verbunden. Analysen der im Kompaktaquerschnitt nur in sehr geringen Konzentrationen vorhandenen Spurenelemente waren daher mit dieser Technik nicht möglich. 
Die für diese Untersuchung relevanten Hauptelemente (Ca, P, $\mathrm{Mg}, \mathrm{Na}$ und $\mathrm{S}$; vgl. 2.4) waren im gesamten Kompaktaquerschnitt in hinreichend hohen bzw. differenzierbaren Konzentrationen enthalten. Für diese Elemente konnten daher kleinräumige Unterschiede der Elementkonzentrationen im Knochenmineral mit der EPMA bestimmt werden.

Die Analysen erfolgten mit einem Gerät des Typs JXA 8900 RL (JEOL). Die Messungen wurden in zwei verschiedenen Analysemodi durchgeführt. Zunächst sind gezielte Einzelpunktanalysen in spezifischen Probenarealen durchgeführt worden. Anschließend wurden Elementverteilungsbilder bzw. Konzentrationsmuster eines Sektors des Kompaktaquerschnittes über die gesamte radiale Ausdehnung erfaßt.

Durch Auswertung von SEM-Bildern (vgl. Abbildung 24) bzw. Abgleich mit zugehörigen lichtmikroskopischen histologischen Aufnahmen (vgl. Abbildung 21) wurde zunächst innerhalb des Kompaktaquerschnittes ein Sektor für die Analyse ausgewählt. Entsprechend dem Versuchsdesign wurde ein Areal mit deutlich variabler Erhaltungscharakteristik des Knochenminerals präferiert (vgl. 2.4).

Innerhalb des gewählten Ausschnittes wurden anschließend knochennative bzw. veränderter Mineralareale angesprochen und in diesen einzelne Meßpunkte vorgewählt $(n=76)$. Dies erfolgte mit der in den EPM-Analyser integrierten elektromechanischen Steuereinheit für den Probentisch (Point-Logger). Die Koordinaten der definierten Meßpunkte wurden gespeichert und anschließend in einer automatisierten Messung vom Point-Logger angesteuert und die Elementgehalte analysiert. Bei diesen Analysen wurden die Konzentrationen weiterer Elemente ( $\mathrm{Al}, \mathrm{Cl}, \mathrm{F}, \mathrm{Fe}, \mathrm{K}, \mathrm{Mn}, \mathrm{Sr}, \mathrm{Si})$ bestimmt. Infolge deren geringer (nahe bzw. unter der Nachweisgrenze) und/oder indifferenter und daher nicht aussagekräftiger Konzentrationen wurden diese Elemente aber von der weiteren Auswertung ausgenommen. Das Element Si wurde ausschließlich für die laborinterne Kontrolle und Charakterisierung des Probeneinbettmediums analysiert.

Die Verteilungsbilder der Elementkonzentrationen von Ca, P, Mg und $\mathrm{Na}$ im Kompaktaquerschnitt wurden anschließend durch die Analyse zweier radial aufeinanderfolgender Flächen von Meßpunkten mit je 920 (radiale Ausdehnung) $\times 300$ (Sektorbreite) und einem Meßpunktraster von 2,5 $\mu \mathrm{m}$ erstellt. Der Kompaktaquerschnitt der analysierten Knochenproben ist damit über seine radiale Gesamtausdehnung (Periost bis Markraum) von ca. 4,6 $\mathrm{mm}$ in einem Sektorstreifen von ca. $0,75 \mathrm{~mm}$ Breite erfaßt worden. 
Die Anzahl der mit hoher Empfindlichkeit simultan detektierbaren Elemente ist geräteseitig auf fünf begrenzt. Als fünftes Element wurde Chlor gewählt. Diese Ergebnisse wurden aus o.g. Gründen im Nachhinein aber als nicht aussagekräftig eingeschätzt und nicht weiter ausgewertet. Durch die Entscheidung für Chlor ist aber kein Verteilungsbild für Schwefel erstellt worden. Als weiteres Element wurde Silizium mit dem weniger sensitiven energiedispersiven Spektrometer (EDS) detektiert. Dies erfolgte im Zusammenhang mit dem neuen Einbettmedium (vgl. 3.2.1.7) ohne weitere Auswertungen im Untersuchungskontext.

Alle Elemente sind sowohl bei den Einzelpunkt- als auch bei den Verteilungsmessungen mit den wellenlängendispersiven Spektrometern (WDS) analysiert worden [Ausnahme: SiAnalyse bei der Verteilungsmessung erfolgte mit dem energiedispersiven Spektrometer (EDS), s.o.]. Für die quantitative Analyse ist die Korrekturmethode von Armstrong (1991) verwendet worden. Die Kalibrierung des Analysesystems erfolgte mit natürlichen Mineralen sowie synthetischen Substanzen mit bekannten Elementoxid- bzw. Elementgehalten. Die Meßparameter für die Einzelpunkt- und Verteilungsanalysen sowie Angaben zu Standardmaterialien sind im Anhang (Tabelle 16 bzw. Tabelle 17) aufgelistet.

Die geräteseitige Ausgabe der quantitativen Analyseergebnisse der Einzelpunktmessungen erfolgte entsprechend der Kalibrierung in Gewichtsprozent der spezifischen Elementoxide (Ausnahme: $\mathrm{Cl}$ und $\mathrm{F}$ in Gewichtsprozent Element). Die für die weitere Auswertung erforderliche Umrechnung in Elementkonzentrationen wird in 3.2.3.1 erläutert.

Bei den Elementverteilungsbildern erfolgte keine Umrechnung der Analysedaten in Elementkonzentrationen. Die innerhalb der analysierten Kompaktafläche variierenden Signalintensitäten (elementspezifische Zählereignisse für jeden Meßpunkt bzw. Meßschritt: counts per step) wurden als Maß für Konzentrationsunterschiede für das jeweilige Element ausgewertet.

\section{LA-ICP-TOFMS}

Neben den mit der EPMA in auswertbaren Konzentrationen gemessenen Hauptelementen (s.o.) wurden in dem Kompaktaquerschnitt die Elemente Strontium, Barium und Uran untersucht. Diese Elemente wurden auf Grund ihrer geringen Konzentrationen mit der LA-ICP-TOFMS analysiert. Als interne Bezugsgröße für die weitere Auswertung wurde wiederum das Matrixelement Calcium mitanalysiert. 
Für diese Messungen wurde ein LA-ICP-TOFMS-Gerätekomplex genutzt. Mit einem Excimer Laser (COMPex ${ }^{\mathrm{TM}}$ 110, Lambda Physik ${ }^{\circledR}$ ) in Kombination mit der Optischen Bank (GeoLas 100 Q, MicroLas Lasersystem) erfolgte die Materialablation von der Probenoberfläche. Die Elementanalysen im abgetragenen Probenmaterial wurden mit einer ICP-TOFMS (LECO Renaissance ${ }^{\mathrm{TM}}$ ) realisiert (zu Anwendungsmöglichkeiten der ICP-MS allgemein vgl. Jarvis \& Jarvis 1992; zur speziellen Anwendung der ICP-MS mit der Laserablation vgl. Simon et al. 1997).

Die drei Elemente wurden hinsichtlich ihrer radialen Konzentrationsgradienten im Kompaktaquerschnitt untersucht (vgl. 2.4). Da diese Spurenelementdaten anschließend mit den Hauptelementergebnissen der EPMA kombiniert interpretiert werden sollten, wurden die Meßpunkte für der LA-ICP-TOFMS-Analysen innerhalb des bei der Elementverteilungsanalyse mit EPMA erfaßten Sektorstreifen festgelegt (s.o.).

Die Meßpunkte im Kompaktaquerschnitt (Punkte für die Laserablation) wurden über ein Lichtmikroskop via CCD-Kamera unter Vermeidung sehr poröser bzw. rissiger Probenareale angesteuert (Anzahl der Meßpunkte im Kompaktaquerschnitt von Periost bis Markraum $n=29$ ). Vor jeder eigentlichen Analyseablation wurden mit zwei Laserpulsen von der Probenoberfläche sowohl die Kohlebedampfungsschicht (vgl. Probenvorbereitung zur EPMA 3.2.1.7) als auch mögliche Materialauflagerungen vorangegangener Ablationen abgetragen (entfernte Materialschicht ca. $2 \mu \mathrm{m}$ ). Die Ablationsdauer für die anschließende Elementanalyse betrug $2 \mathrm{~s}$. Bei einer Laserpulsfrequenz von $5 \mathrm{~Hz}$ resultierten pro Meßpunkt zehn Laserpulse und somit der Abtrag einer Materialschicht von $10 \mu \mathrm{m}$ von der Kompaktaoberfläche. Die hier bereits genannten Parameter einschließlich weiterer für die Durchführung der LA-ICP-TOFMS-Analysen relevanten geräteseitigen Einstellungen sind im Anhang (Tabelle 18) aufgelistet.

Da für die Auswertung der sehr geringen Konzentrationen der untersuchten Spurenelemente auch mögliche Elementgehalte im Einbettungsmedium zu berücksichtigen waren, wurden zum Vergleich auch Ablationsmeßpunkte in das Einbettmedium gelegt.

Für die geräteseitige Analyseoptimierung, -kalibrierung und -kontrolle wurde der Glas-Standard SRM 610 (Standard Reference Material 610 Silicate Glass, National Institute of Standards \& Technology, Gaithersburg) gemessen.

Infolge des variierenden Materialabtrags durch den Laser am jeweiligen Ablationsort ist methodenbedingt keine Bezugsmasse für eine Konzentrationsberechnung bestimmbar. Die 
Auswertungen dieser Analysen erfolgt daher ausschließlich meßpunktvergleichend auf der Ebene der Meßsignale (vgl. 3.2.3.1, S 61). Für die drei Spurenelemente sowie das Matrixelement $\mathrm{Ca}$ wurden hierbei die Meßsignale für jeweils eines ihrer Isotope ausgewertet $\left({ }^{88} \mathrm{Sr},{ }^{137} \mathrm{Ba},{ }^{238} \mathrm{U},{ }^{44} \mathrm{Ca}\right)$. Für die Elemente $\mathrm{Ca}$ und $\mathrm{Sr}$ erfolgte die Auswertung der Stromstärke der Meßsignale bzw. eines entsprechendem Spannungsabfall an einem Meßwiderstand in $\mathrm{mV}$. Die Meßsignale für $\mathrm{Ba}$ und $\mathrm{U}$ wurden als counts per second (cps) ausgewertet.

Bei diesen Analysen sind methodenimmanent innerhalb des detektierten Massenbereichs von Li bis $\mathrm{U}$ die Meßsignale für weitere Elemente respektive Isotope erfaßt worden. Die Auswertungen wurden aber auf fragestellungsrelevante und aussagekräftige Elementkonzentrationen beschränkt. $\mathrm{Zu}$ beachten ist, daß insbesondere auch das Element Al analysiert wurde. Ein sehr hoher Background-Gehalt (höher als die Knochengehalte) der Kontrollmessung im Einbettungsmedium erforderte aber auch für diese Daten den Ausschluß von der Auswertung.

\subsubsection{Datenauswertung und Statistik}

\subsubsection{Konzentrationsberechnungen}

Aus den vorangegangenen Kapiteln bezüglich der angewendeten verschiedenen Analysetechniken geht hervor, daß die resultierenden Meßdaten methodenabhängig in unterschiedlicher Form vorlagen. Diese Rohdaten erforderten für die verschiedenen Teilaspekte der Untersuchung eine methodenspezifische Umrechnung in vergleich- und interpretierbare Elementgehalte.

Für die Meßtechniken zur Konzentrationsbestimmung in Lösung (AAS, ICP-OES und Photometrie) ist das Berechnungsprinzip prinzipiell gleich.

\section{Konzentrationsberechnung für AAS, ICP-OES und photometrische Bestimmung}

Bei den spektrometrischen Analysetechniken werden die Meßsignale entsprechend der Gerätekalibration als Konzentrationen der gemessenen Lösungen (verdünnte Probenstammlösung) ausgegeben. Diese Elementgehalte in $\mathrm{mg} / \mathrm{l}$ (bzw. $\mu \mathrm{g} / \mathrm{ml}$ ) bei der AAS und $\mu \mathrm{g} / 1$ bei der ICP-OES wurden auf Elementkonzentrationen in den Knochenproben entsprechend dem allgemeinen Berechnungsprinzip umgerechnet (vgl. Schutkowski 1994). 
Die Berechnungsgrundlage bilden die gemessene Meßlösungskonzentration $\left(\mathrm{C}_{\text {Meßlösung }}\right)$, das Volumen der Probenstammlösung ( $V_{\text {Probenstammlösung }}$ ), der sich aus dem Meßlösungsvolumen $\left(\mathrm{V}_{\text {Meßlösung }}\right)$ und dem in diesem enthaltenen Probenvolumen $\left(\mathrm{V}_{\text {Stammlösungsanteil }}\right)$ ergebende Verdünnungsfaktor sowie die eingewogene Knochenmasse ( $m_{\text {Einwaage). Die }}$ Berechnung der Probenkonzentration $\left(\mathrm{C}_{\text {Probe }}\right)$ erfolgte nach folgender Gleichung:

$\mathrm{C}_{\text {Probe }}[\mu \mathrm{g} / \mathrm{g}]=\frac{\mathrm{C}_{\text {Meßlösung }}[\mu \mathrm{g} / \mathrm{ml}] \bullet \mathrm{V}_{\text {Probenstammlösung }}[\mathrm{ml}] \bullet \frac{\mathrm{V}_{\text {MeßBlösung }}[\mathrm{ml}]}{\mathrm{V}_{\text {Stammlösungsanteil }[}[\mathrm{ml}]}}{\left.\text { mEinwaage }_{\mathrm{g}}\right]} \quad$ Gleichung 1

Der Berechnung mit dem angegebenen Einheitensatz entsprechend wurden die Meßdaten der ICP-OES zuvor von $\mu \mathrm{g} / \mathrm{l}$ in $\mu \mathrm{g} / \mathrm{ml}$ umgerechnet.

Dem Verdünnungsfaktor als Quotient aus $\left(\mathrm{V}_{\text {Meßlösung }}\right)$ und $\left(\mathrm{V}_{\text {Stammlösungsanteil }}\right)$ entsprechen bei der AAS-Analytik die Angaben zur Verdünnung im Anhang (Tabelle 12). Aus der simultanen Analyse aller Elemente mit der ICP-OES resultiert bei dieser für alle Elementberechnungen eine einheitlicher Verdünnungsfaktor (1:7). Da die Einwaagemassen zwischen 60-100 mg lagen, ist zu beachten, daß für eine hinreichende Genauigkeit m $\mathrm{m}_{\text {Einwaage }}$ auf $0,01 \mathrm{mg}$ genau in die Berechnungen eingegangen ist.

Die Konzentrationsangaben erfolgen für die Spurenelemente Ba und $\mathrm{Sr}$ in $\boldsymbol{\mu g}$ Elementmasseanteil pro $\mathbf{g}$ Probenmasse. Für die Matrixelemente $\mathrm{Ca}$ und $\mathrm{P}$ wird $\mathrm{C}_{\text {Probe }}$ in $\mathbf{m g} / \mathbf{g}$ angegeben.

Für Phosphor ist bezüglich der ICP-OES anzumerken, daß die Meßwerte als Phosphatkonzentration angegeben waren (entsprechend der Kalibrierlösungen mit definierten $\mathrm{PO}_{4}$-Gehalten, vgl. Anhang, Tabelle 15). Diese Meßwerte bedurften daher zunächst der Umrechnung auf die Phosphorkonzentrationen der Meßlösungen, bevor Gleichung 1 angewendet werden konnte. Der Umrechnungsfaktor (-divisor) resultiert aus dem Masseanteil von Phosphor an der Phosphatmasse entsprechend der Phosphatstöchiometrie (vgl. Anhang, Tabelle 19).

Die Phosphorkonzentrationen wurden aber nur mit der ICP-OES-Technik spektrometrisch bestimmt. Bei den früheren AAS-Analysen erfolgte die Bestimmung über zusätzliche photometrische Messungen. Die Kalibrierung erfolgte hierbei nicht geräteintern (photometerintern). Daher erfolgte die Meßwertausgabe nicht als Konzentration der Meßlösung sondern nur als Extinktionswert. Mit analog den Meßlösungen vorbereiteten Kalibrierlösungen definierter Konzentration wurde eine Kalibriergerade (Regressionsgerade von Extinktionswerten und zugehörigen Konzentrationen der Kalibrierlösungen) erstellt und 
regelmäßig kontrolliert bzw. aktualisiert. Mit der Gleichung dieser Kalibriergeraden konnten aus den Extinktionswerten der photometrischen Messungen die Meßlösungskonzentrationen $\left(\mathrm{C}_{\text {Meßlösung }}\right)$ berechnet werden. Diese wurden zur Berechnung der Knochenkonzentrationen abschließend wieder in Gleichung 1 eingesetzt.

\section{Konzentrationsberechnung für EPMA}

Die Meßdaten dieser Analysen werden geräteseitig als Konzentrationen ausgegeben, die bereits primär auf die Knochenmasse bezogen sind. Da diese Meßwertausgabe aber als Gewichtsprozent der Referenzverbindungen bzw. -oxide erfolgt, war eine Umrechnung in Elementkonzentrationen erforderlich.

Grundlage hierfür bildeten wieder die aus der Stöchiometrie der jeweiligen Elementverbindung resultierenden Masserelationen zwischen den Elementen und ihren Verbindungen (vgl. Berechnung der Phosphorkonzentrationen bei ICP-OES). Für die Elemente Ca und P wurde hierbei die Ausgangseinheit Gewichtsprozent (entspricht mg/g) beibehalten. Für die anderen ausgewerteten Elementkonzentrationen ( $\mathrm{Mg}, \mathrm{Na}, \mathrm{S}$ ) erfolgte im Zuge dieser Berechnungen eine Einheitenumrechnung von Gewichtsprozent in $\mu \mathrm{g} / \mathrm{g}$. Für die ausgewerteten Elemente sind Stöchiometrie, relative Massen und resultierende Umrechnungsfaktoren (-divisoren) im Anhang (Tabelle 19) aufgelistet.

Bei den mit der EPMA gemessenen bzw. berechneten Absolutkonzentrationen der Elemente ist deren Bezug auf die die gesamte Knochenmasse zu beachten. Letztere beinhaltet auch den organischen Anteil der Knochensubstanz, da die untersuchte Knochenprobe nicht vorher verascht wurde. Gleichzeitig gestattet aber die durch die EPMA bestimmte Mineralsumme einen Rückschluß auf den nichtmineralischen Anteil.

Wie im nächsten Abschnitt geschildert wird, ist durch die gewählte Form der Datenauswertung die Vergleichbarkeit der Daten unabhängig von einem in der Probe enthaltenen organischen Anteil prinzipiell gewährleistet.

\section{Umrechnung Absolutkonzentrationen in Konzentrationsquotienten}

Im einleitenden Kapitel dieser Arbeit wurde die Problematik des Vergleichs von Absolutkonzentrationen in der Knochensubstanz bereits verdeutlicht. Es wurde gezeigt, daß der theoretische Ansatz zur Interpretation von Elementgehalten in Knochenproben Konzentra- 
tionsquotienten als Auswertungsbasis präferiert (vgl. 1.1). Daher erfolgte in der vorliegenden Arbeit in allen Teiluntersuchungen die Auswertung anhand von probenintern berechneten Konzentrationsquotienten. Die Elementgehalte wurden generell auf das Knochenmatrixelement Ca bezogen. Aus der Berechnung der Quotienten von Spurenelementkonzentrationen in $\mu \mathrm{g} / \mathrm{g}$ und Calciumkonzentrationen in $\mathrm{mg} / \mathrm{g}$ resultieren entsprechend kleine Werte. Diese Quotientendaten werden daher grundsätzlich als Zehnerpotenz $\left(10^{-3}\right)$ angegeben. In allen Abbildungen und Tabellen ist ein Vermerk dieser Größenordnung enthalten (Spaltenüberschrift bzw. Skalenbeschriftung).

Ausgenommen von dieser Form der Quotientenbildung ist als weiteres Knochenmatrixelement Phosphor. Den theoretischen Vorbemerkungen in 2.3 (S. 28f) entsprechend erfolgt hier gegenüber den anderen Elementen der reziproke Bezug von $\mathrm{Ca}$ auf $\mathrm{P}(\mathrm{Ca} / \mathrm{P})$, der als ein mineral-chemisch charakteristischer Wert ausgewertet wird.

\section{Quotientenberechnung für LA-ICP-TOFMS}

Für diese Analysetechnik erfolgte keine Umrechnung der Meßsignale in Konzentrationswerte. Da für die Auswertung nur die probeninterne Variabilität der Elementgehalte von $\mathrm{Ba}$, Sr und U relevant waren, genügte eine probeninterne (meßpunktinterne) Normierung der Meßsignale dieser Element auf das Meßsignal von Calcium als Matrix- bzw. Bezugselement. Hierbei wird das Ca-Meßsignal als Referenz für den Masseabtrag durch die Ablation bzw. den darin enthaltenen Mineralanteil ausgewertet.

Die resultierenden Elementsignal-Quotienten sind als Maß für die Elementgehalte der einzelnen Meßpunkte vergleichbar und konnten somit hinsichtlich ihrer Unterschiede ausgewertet werden.

\subsubsection{Referenzmessungen, Fehlerberechnung und Nachweisgrenzen}

\section{Referenzmessungen}

Für die Kontrolle der Meßqualität bzw. zur Fehlerabschätzung waren die Konzentrationsmessungen für die Pauschalanalytik in Lösung (AAS und ICP-OES) von Kontrollmessungen entsprechender Standardreferenzmaterialien begleitet. Durch die statistische Auswertung wiederholter Messungen dieser Referenzmaterialien wurde sowohl die Richtigkeit als auch die Präzision der Analysemethoden erfaßt. 
Über den Vergleich des Mittelwertes der in den Referenzmessungen gemessenen Konzentrationen eines Elementes mit seinem für das Referenzmaterial zertifizierten Wert erfolgt die Überprüfung der Richtigkeit. Entsprechend werden die Referenzkonzentrationen bei den Auswertungen der Referenzmessungen mit aufgeführt (vgl. Anhang, Tabelle 20).

Für die vorliegende Untersuchung besitzt aber die Präzision (Reproduzierbarkeit) der Analysen größere Relevanz, da nur die Vergleichbarkeit der Messwerte im jeweiligen Untersuchungskontext gewährleistet sein muß (vgl. 2). Die Referenzmaterialien werden daher hinsichtlich der aus den wiederholten Messungen der Elementkonzentrationen resultierenden relativen Standardabweichungen ausgewertet. Grundlage hierfür bildet die absolute Standardabweichung ausgehend von einer Stichprobe.

Als wichtigstes Referenzmaterial wurde das Standard Reference Material 1400 - Bone Ash (SRM 1400, National Institute of Standards \& Technology, Gaithersburg) verwendet. Da es für diesen Standard aber keinen zertifizierten Bariumkonzentrationswert gibt, wurde bei den früheren AAS-Analysen für die Bariumbestimmung als Referenzmaterial der Standard Animal Bone H-5 (International Atomic Energy Agency, Wien) gemessen. Für die Analysen des Gräberfeldes Kirchheim unter Teck mit ICP-OES wurde ausschließlich das letztgenannte Referenzmaterial verwendet. Im Hinblick auf die oben genannte Präferenz der Präzision gegenüber der Richtigkeit wurde auf ein Referenzmaterial mit zertifiziertem Bariumgehalt verzichtet. Die Qualität der Bariummessungen wurde hierbei nur über die laborinterne Reproduzierbarkeit der im SRM 1400 bestimmten Bariumgehalte überprüft.

Die Referenzmaterialien wurden den Angaben in ihren Zertifikaten entsprechend vorbereitet. Ihre Überführung in Lösung erfolgte analog der Herstellung der Stammlösungen der Knochenproben (vgl. 3.2.1.4). Die für die Analysen variierenden Verdünnungen dieser Stammlösungen waren wiederum gleich denen der Knochenproben (vgl. Anhang, Tabelle 12). Die Umrechnung der in den Meßlösungen der Referenzmaterialien bestimmten Konzentrationen auf den Elementgehalt ihrer Festsubstanz entspricht daher den Konzentrationsberechnungen für die Knochenproben (vgl. 3.2.3.1).

In die Meßreihen der AAS-Analysen (für $\mathrm{Ca}, \mathrm{Ba}, \mathrm{Sr}$ ) wurde jeweils nach sechs Probenmessungen eine Referenzmaterialmessung integriert. Die Referenzmessungen für die photometrische Phosphorbestimmung wurden nach jeder zehnten Probenanalyse durchgeführt. Bei der ICP-OES-Analytik (für Ca, P, Sr, Ba, Mg, Al, Na) wurden jeweils 35 Probenmessungen von vier Referenzmessungen begleitet. 
Fehlerberechnung für AAS und ICP-OES sowie photometrische Phosphorbestimmung

Im Rahmen der Auswertungen der Daten werden, wie bereits mehrfach erwähnt, nur Konzentrationsquotienten interpretiert. Für die Untersuchung sind daher auch nur deren Fehlergrößen relevant. Hieraus ergibt sich die Notwendigkeit, aus den Fehlern der Analysen der Elementkonzentrationen über die Gaußsche Fehlerfortpflanzung die für die Konzentrationsquotienten resultierenden Fehler zu ermitteln. Aus den relativen Fehlern (relative Standardabweichungen) der Referenzmessungen der Konzentrationen zweier Elemente X und Y resultiert als relativer Gesamtfehler für die Bestimmung des Konzentrationsquotienten die Wurzel aus der Summe der Quadrate beider relativer Fehler:

$$
\text { relativer Fehler }\left(\frac{X}{Y}\right)=\sqrt{(\text { relativer Fehler } X)^{2}+(\text { relativer Fehler } Y)^{2}}
$$

Gleichung 2

Für die wiederholten Referenzmessungen waren mehrere Aufschlüsse (Stammlösungen) der Referenzmaterialien erforderlich. Die Messungen dieser verschiedenen Aufschlüsse sind aber alle gemeinsam in die Fehlerberechung eingegangen. Hierdurch wurden in den ermittelten relativen Fehlern auch mögliche zufällige Fehler (Einwaage- und Verdünnungsfehler) bei der Herstellung von Stammlösungen erfaßt.

Die Auswertungen der Referenzmessungen sind im Anhang aufgelistet (Tabelle 21 und Tabelle 22).

\section{Fehlerschätzung für EPMA}

Für diese Technik wurden die relativen Fehler der Elementanalysen eines Meßpunktes mit typischen (mittleren) Elementkonzentrationen als Fehlerreferenz verwendet. Aus diesen elementbezogenen Einzelfehlern ergeben sich die Gesamtfehler für die auswertungsrelevanten Konzentrationsquotienten wiederum aus der Gaußschen Fehlerfortpflanzung entsprechend Gleichung 2 (vgl. Anhang, Tabelle 24).

Bei einer sehr geringen Elementkonzentration (nahe der Nachweisgrenze) kann der relative Fehler für das entsprechende Element sehr hoch werden. Infolge der dann jedoch geringen Absolutkonzentration folgen hieraus trotzdem nur geringe und damit für die Auswertung nicht relevante Absolutfehler. Gleiches gilt für die resultierenden Fehler der Konzentrationsquotienten. 


\section{Fehlerschätzung für LA-ICP-TOFMS}

Auf der Grundlage laborinterner Erfahrungen wird bei diesen Analysen für die Messung einzelner Elemente respektive Isotope ein Fehler von 20\% angenommen (Simon et al. 1997; Simon, persönliche Mitteilung). Auf der Grundlage der Gaußschen Fehlerfortpflanzung (vgl. Gleichung 2) folgt für Quotienten ein Gesamtfehler von rund 30 \%.

Allgemein kann bezüglich der Analysefehler festgestellt werden, daß mit allen verwendeten Analysemethoden die Calciumkonzentrationen mit hoher Präzision (geringe relative Fehler) bestimmt werden konnten. Die Fehler für die Konzentrationsquotienten werden daher hauptsächlich durch die Fehler der Konzentrationsbestimmungen der auf Calcium bezogenen Elemente bestimmt (vgl. Tabelle 21 bis Tabelle 24, S. 164f).

\section{Nachweisgrenzen für ICP-OES}

Da für die AAS-Analysen die Verdünnungen der Probenstammlösungen jeweils optimalen Analysebedingungen angepaßt wurden, brauchten Nachweisgrenzen hierbei nicht berücksichtigt zu werden.

Für die simultane Elementanalytik mit ICP-OES wurde ein einheitlicher Verdünnungsfaktor verwendet. Daher war nicht immer für alle analysierten Elemente ein Verdünnungsoptimum realisierbar. Folglich sind bei diesen Analysen die Nachweisgrenzen für die Elemente zu beachten. Für das verwendete Analysegerät waren laborintern empirisch geprüfte Nachweisgrenzen bekannt. Analog den Konzentrationsberechnungen (vgl. 3.2.3.1) wurden diese Nachweisgrenzen für die Meßlösung auf Nachweisgrenzen für die Knochenproben umgerechnet. Berechnungsgrundlage bildeten die für die ICP-OES-Messungen verwendete Verdünnung der Probenstammlösung und die durchschnittliche Knochenprobeneinwaage (vgl. Tabelle 23). Meßsignale unterhalb der Nachweisgrenze der Meßlösung können zunächst nur als Knochenkonzentrationen unterhalb der auf die Knochensubstanz umgerechneten Grenze interpretiert werden. Solche Meßwerte gingen dann in die Berechnung der Konzentrationsquotienten als Nullwerte ein. Für die Quotienten resultieren entsprechend ebenfalls Nullwerte. Dieses Problem ergab sich nur bei einigen Proben von Kirchheim am Ries für Aluminium sowie bei den Proben des rezenten Knochenmaterials für Aluminium und Barium. 


\section{Nachweisgrenzen für EPMA}

Nachweisgrenzen sind bei der EPMA-Technik substratspezifisch und müssen daher für die jeweilige Probe bestimmt werden. Analog der Fehlerschätzung sind die Nachweisgrenzen für diese Technik auf der Grundlage der Datenauswertung eines Meßpunktes mit typischen Elementkonzentrationen ermittelt worden. Als Nachweisgrenze eines Elementes wurde die Konzentration berechnet, die dem Dreifachen des zählstatistischen Fehlers $[\sqrt{(\text { Zählereignisse })}]$ seines Backgroundsignals entspricht. (vgl. Anhang, Tabelle 24).

\section{Nachweisgrenzen für LA-ICP-TOFMS}

Für die Laserablationsanalysen bildete die Kontrollmessung des Einbettmediums des Knochenstückes (vgl. 3.2.1.7 und 3.2.2.2, S. 57) die Limitierung für die Signalauswertung. Bei den auszuwertenden Elementen wurden Meßsignale, die niedriger als dieser Signalhintergrund waren, als Null gewertet. Entsprechend resultieren für die jeweiligen Signalquotienten (aus Elementsignal und Calciumsignal) ebenfalls Nullwerte. Da Uran im Einbettmedium nicht detektiert wurde und die im Knochen gemessenen Signale teilweise auch sehr niedrig waren, ist für dieses Element als Grenze für den Nullwert ein Signal von 10 cps festgelegt worden (vgl. Anhang, Tabelle 34).

\subsubsection{Datenverwaltung und statistische Software}

Alle Daten und Parameter für die erforderlichen Konzentrations- bzw. Quotientenberechnungen sind im Tabellenkalkulationsprogramm EXCEL 2000 (Microsoft 1999) gespeichert worden. Dies ermöglichte die Durchführung aller Berechnungen innerhalb dieses Datensystems unter Verwendung selbstdefinierter Rechenverknüpfungen. Diese Form der Datenauswertung gewährleistete die ständige Verfügbarkeit der Rohmeßdaten. Die Transparenz der Berechnungen infolge der internen Verknüpfungen der einzelnen Auswertungsschritte trug zur Verbesserung der Datensicherheit durch eine erleichterte Kontrolle und Fehlerkorrektur bei.

Nach Abschluß aller erforderlichen Umrechnungen der Analysedaten in auswertbare Konzentrationen bzw. Quotienten wurden die Daten für die weitere statistische Auswertung in StATISTICA 5.1 (StatSoft 1998) importiert. Mit diesem Programm wurden die im folgenden Kapitel genannten statistischen Auswertungen einschließlich der graphischen Darstellun- 
gen realisiert (mit Ausnahme der nichtparametrischen Varianzanalysen, vgl. S. 69f) Für die Berechnungsalgorithmen der jeweiligen statistischen Testverfahren stellt daher prinzipiell das Handbuch dieses Programms bzw. das Programm selbst die Referenz dar.

\subsubsection{Statistische Verfahren}

Im Folgenden werden die in dieser Untersuchung verwendeten statistischen Verfahren kontextbezogen aufgeführt. Vorangestellt werden Bemerkungen zur Ausreißerproblematik. Es schließen sich die Verfahren zur Prüfung der Verteilungscharakteristika (Normalverteilung, Varianzhomogenität) von Meßdaten an, da diese den Ausgangspunkt für die Entscheidung zwischen parametrischen und nichtparametrischen Testroutinen bilden. Die verwendeten vergleichs- und zusammenhangsbezogenen statistischen Prozeduren werden danach entsprechend der aus Fragestellung bzw. Versuchsdesign resultierenden Hypothesenstruktur aufgeführt.

\section{Ausreißer und Extremwerte}

Für biologische Meßdaten bzw. Meßdaten von biologischen Substanzen ist der auf "Ausreißer" allgemein bezogene Imperativ: "A point is never to be excluded on stastical grounds alone" (Fisher 1922) besonders zu beachten. Für einen Ausschluß extremer Spurenelementdaten von den Auswertungen bedarf es hiernach einer sachlogischen Begründung. Die biologische bzw. biochemische Variabilität schließt aber prinzipiell einen großen Bereich der Merkmalsausprägung ausdrücklich ein. Gleichfalls sind bei bodenchemischen Prozessen stochastische Phänomen mit resultierenden Extremkonzentrationen nicht auszuschließen. Dies und die Komplexität biochemisch-geochemischer Wechselwirkungen erlaubte keine Definition von Bereichen für wahrscheinliche Elementkonzentrationen. Für die anderen quantifizierten und auswertungsrelevanten Daten der Vorbereitung der Knochenproben (Masseverluste) ist eine solche Limitierung aus den gleichen Gründen ebenfalls nicht möglich. Es ermangelt somit prinzipiell einer sachlogischen Ausreißerdefinition. Jegliche dergestalte Datenreduktion wäre also willkürlich. Daher wurden in die Gruppenvergleiche grundsätzlich alle Werte der jeweiligen Gruppen einbezogen. (Diese Aussage ist nicht zu verwechseln mit dem vollständigen Ausschluß der Gruppe der Knochenproben mit mittlerem histologischer Erhaltungszustand; vgl. 2.1, S. 13) 
In den Grafiken zur deskriptiven Statistik der Datengruppen (Boxplots) werden dennoch über die Standardabweichung (s) "statistisch" definierte Ausreißer (Werte außerhalb $\pm 2 \mathrm{~s}$ aber innerhalb $\pm 3 \mathrm{~s}$ ) und Extremwerte (Werte außerhalb $\pm 3 \mathrm{~s}$ ) speziell dargestellt (hinsichtlich der Wertebegrenzungen vgl. Empfehlungen in StatSoft 2001). Dies ermöglicht die Abschätzung der meist geringen Bedeutung dieser wenigen Werte für die Verteilungscharakteristik bzw. die zentrale Tendenz der jeweils dargestellten Wertegruppe.

Besondere Beachtung verlangen mögliche Ausreißer jedoch bei den Korrelationsanalysen, da sie sehr starken Einfluß auf den Korrelationskoeffizienten haben können (vgl. Bortz 1999, S. 206). Dieser Aspekt wird im Abschnitt zu den Korrelationsanalysen nochmals aufgegriffen werden (vgl. S. 71).

\section{Prüfung von Testvoraussetzungen}

Für die statistischen Auswertungen galt es zunächst zu entscheiden, inwieweit die Verwendung parametrischer Statistiken möglich ist oder ob nichtparametrische Verfahren erforderlich sind.

Für die Anwendung parametrischer Testverfahren ist die Normalverteilung die primäre Voraussetzung. Eigentlich auf die zu testenden statistischen Kennwerte bezogen, wird dieses Kriterium anhand der Verteilung der untersuchten Stichprobendaten geprüft. Hierfür wird nur eine Augenscheinprüfung empfohlen. In der vorliegenden Untersuchung sind die Werteverteilungen aber prinzipiell mit dem Shapiro-Wilk's $W$-Test hinsichtlich ihrer Normalverteilung getestet worden.

Da in den verschiedenen Aspekten der Fragestellung schwerpunktmäßig Unterschiedshypothesen getestet wurden, war die Normalität der Daten für jede der in den Vergleich eingebundenen Einzelgruppe zu prüfen. Als weiterer Voraussetzung zwischen den zu vergleichenden Einzelgruppen sollte Varianzhomogenität bestehen. Die diesbezüglichen Überprüfungen wurden mit dem Levene-Test vorgenommen. Für die mehrfaktoriellen Vergleichsdesigns erfolgten die Überprüfungen für die Abweichungswerte innerhalb bzw. zwischen den Zellen aller Faktorgruppen.

Die Testergebnisse zu den Verteilungscharakteristika aller auswertungsrelevanten Variablen der zu vergleichenden Einzelgruppen sind im Anhang zusammen mit den statistisch deskriptiven Daten gelistet (ab Tabelle 37, S. 179ff). Zu beachten ist, daß bei den Prüfungen der Normalität hinsichtlich eines geringeren $\beta$-Fehler-Risikos $\boldsymbol{\alpha}=\mathbf{0 , 2 5}$ festgesetzt wur- 
de, d.h. wenn aus dem Shapiro-Wilk's W-Test $\mathrm{p}<0,25$ resultiert, die entsprechende Verteilung bereits als signifikant von der Normalverteilung abweichend eingeschätzt wurde.

\section{Entscheidung zwischen parametrischen und nichtparametrischen Verfahren zur Prüfung von Unterschiedshypothesen}

Aus den Prüfungen der Testvorbedingungen resultierten für die Datenverteilungen der einzelnen Variablen innerhalb der zu vergleichenden Gruppen sehr heterogene Charakteristiken. Teilweise sind alle Voraussetzungen erfüllt. Jedoch sind in verschiedenen Gruppen für einzelne Variablen Normalität und/oder Varianzhomogenität nicht gewährleistet. Auch eine bezüglich Spurenelementdaten in der Literatur empfohlene Datentransformation (Logarithmierung, vgl. z.B. Buikstra et al. 1989) führte nicht konsistent zur hinreichenden Angleichung aller Datenverteilungen an die Normalität.

Prinzipiell gelten alle parametrischen Vergleichsverfahren [t-Test, ein- bzw. mehrfaktorielle ANOVA (analysis of variance)] gegenüber den Verletzungen einzelner Voraussetzungen als robust. Jedoch wird vor der Kombination mehrerer Verletzungen gewarnt. Bei nichtvorhandener Varianzhomogenität wird zusätzlich auf die Probleme bei positivem Zusammenhang zwischen Varianzen und Stichprobenmittelwerten bzw. bei negativem Zusammenhang zwischen Varianzen und Stichprobenumfängen verwiesen. Durch unterschiedliche Größen der zu vergleichenden Stichproben (unbalancierte Vergleiche) werden diese Probleme einer möglichen Verfälschung der Testergebnisse verschärft. Es wird insbesondere darauf hingewiesen, daß auch nach Transformationen der Rohdaten zur Anpassung an die Normalität gerade die ANOVA zu Fehlbewertungen neigt (vgl. Thompson 2000, S. 175; zur Problematik der mathematischen Voraussetzungen für statistische Testverfahren vgl. Bortz et al. 2000, S. $81 \mathrm{ff}$ bzw. Bortz 1999, S. $273 \mathrm{ff}$ und 317 sowie StatSoft 2001).

Kombinierte Verletzungen der Voraussetzungen waren für geplante Vergleiche gegeben und verfälschte Testergebnisse daher nicht auszuschließen. Für die Interpretation der Ergebnisse war die Vergleichbarkeit der aus den Statistiken abgeleiteten Aussagen relevant. Die Möglichkeit, den Voraussetzungen entsprechend teilweise parametrisch und teilweise nichtparametrisch zu testen, wurde daher verworfen. Zur Prüfung aller Unterschiedshypothesen sind nichtparamtrischen Verfahren gewählt worden. 


\section{Verwendete Verfahren zur Prüfung von Unterschiedshypothesen}

Gegenüberstellung möglicher Gruppenfaktoren innerhalb der Population von Weingarten:

Die Hypothesen zur Gruppenstruktur innerhalb der Population von Weingarten (vgl. 2.1, S. 19ff) entsprechen einem dreifaktoriellen Varianzanalyse-Design. Geprüft wird der Einfluß von drei Faktoren mit je zwei Stufen (Geschlecht: männlich/weiblich; Sozialstatus: niederer/hoher Rang; Histologie: gut/schlecht) auf zwei Variablen $(\mathrm{Sr} / \mathrm{Ca}$ und $\mathrm{Ba} / \mathrm{Ca})$. Als Interaktion wird entsprechend der dort erläuterten Hypothesenstruktur nur die Kombination Geschlecht-Sozialstatus in den Auswertungen berücksichtigt. Die Analyse ist für jede abhängige Variable separat (univariat) durchgeführt und interpretiert worden. Verwendet wurde ein nichtparametrisches Verfahren zur Varianzanalyse [ATS (ANOVA-type statistic); Akritas et al. 1997 bzw. Brunner \& Puri 2001].

Vergleich der Auswirkungen der Aufreinigungsmethoden auf die beiden histologischen Gruppen:

Die Untersuchungen zur Aufreinigungsmethode (vgl. 2.2) entsprechen einem Versuchsdesign mit Meßwiederholung. Die in diesem Zusammenhang geprüften Variablen ( $\mathrm{Sr} / \mathrm{Ca}$, $\mathrm{Ba} / \mathrm{Ca}, \mathrm{Al} / \mathrm{Ca}, \mathrm{Mg} / \mathrm{Ca}$ und Masseverlust beim Veraschen) sind in vier Wiederholungen gemessen worden (ohne Probenaufreinigung sowie nach drei verschiedenen Aufreinigungsmethoden). Die entscheidende Hypothesenprüfung galt hierbei der Frage nach einer unterschiedlichen Beeinflussung der histologischen Gruppen durch die Probenvorbehandlung. In eine Meßwiederholungshypothese übersetzt, ist dies die Frage nach einem Unterschied zwischen den Änderungen für die beiden histologischen Gruppen während der Meßwiederholungen. Es wird die Signifikanz der Interaktion zwischen den Faktoren Histologie und Probenvorbehandlung geprüft. Die Hypothesenstruktur entspricht damit dem Split-Plot Design. Die Meßwiederholungen werden für einen in zwei Gruppen "gesplitteten Plot" miteinander verglichen. Die Analysen wurden univariat durchgeführt. Das verwendete nichtparametrische varianzanalytische Verfahren leitet sich von der oben genannten ATS ab (vgl. Brunner \& Puri 2001). Entsprechend der kleinen Stichproben wurde die BoxApproximation verwendet (vgl. Akritas et al. 1997).

Die beiden genannten varianzanalytischen Statistiken konnten nicht mit der StATISTICA-Software durchgeführt werden. Es wurden entsprechende Module des SASProgrammsystems genutzt (Programmversion 8.0 mit Programmiersprache SAS/IML ${ }^{\circledR}$; 
SAS Institute 2000). Hierfür erfolgte zunächst mit STATISTICA eine Umstrukturierung des auszuwertenden Datensatzes in eine nach Faktorkombinationen (Zellen) sortierte Datenmatrix. Nach Konvertierung in eine Textdatei (ASCII) konnte diese als Eingabedatei für das SAS-Modul genutzt werden (zu dieser softwaregestützten Realisierung der Statistiken vgl. Brunner \& Puri 2001). Die Berechnungen wurden in der Abteilung Medizinische Statistik der Universität Göttingen durchgeführt.

Einzelvergleiche für mehrere Variablen der histologischen Gruppen von Kirchheim unter Teck Wenigumstadt und Kirchheim am Ries sowie die Einzelpunktanalysen mit EPMA:

Für die Populationen Kirchheim unter Teck und Wenigumstadt waren die Unterschiedshypothesen auf den Gruppierungsfaktor Histologie ohne Gegenüberstellung mit möglichen alternativen Faktoren reduziert (vgl. 2.1, S. 21f). Hieraus resultiert für die statistische Auswertung der Variablen nur noch ein einfaktorielles Vergleichsdesign. Für jede Variable wird die Hypothese eines Gruppenunterschiedes über einen Einzelvergleich getestet. Gleiches gilt für all jene Variablen von Weingarten $(\mathrm{Ca} / \mathrm{P}$, Masseverlust beim Ätzen, Masseverlust beim Veraschen) bzw. Kirchheim am Ries (Ca/P, Masseverlust beim Ätzen) die hypothesenbedingt nicht in die Varianzanalysen integriert wurden. Die Gruppenvergleiche der Einzelpunktanalysen mit der EPMA (native vs. veränderte Mineralareale) erfolgten für alle auswertungsrelevanten Variablen ebenfalls über Einzelvergleiche.

Als nichtparametrisches Verfahren für den Vergleich zweier unabhängiger Stichproben wurde der Mann-Whitney-U-Test gewählt. Da bei den Gruppenvergleichen mindestens für eine der beiden Gruppen die Probenanzahl größer als 20 war, bzw. die kleinere Gruppe nie weniger als 18 Proben enthielt erfolgte die Prüfung über den aus dem U-Wert berechneten Z-Wert.

Der Vergleich der sehr kleinen Stichprobe von $\mathrm{Na} / \mathrm{Ca}$-Werten für Kirchheim am Ries wurde mit dem Kolmogorov-Smirnov-Test durchgeführt. Dies entspricht der Empfehlung von Siegel (1987), diesen Test für kleine Stichproben dem Mann-Whitney-U-Test vorzuziehen. 


\section{Verwendetes Verfahren zur Prüfung von Zusammenhangshypothesen}

Im Hypothesenkonzept dieser Untersuchung ist die Prüfung des Zusammenhanges zwischen zwei Variablen enthalten. Es wurden jeweils für die Gruppe der histologisch gut und die der histologisch schlecht erhaltenen Knochenproben die gruppeninternen Korrelationen der $\mathrm{Ba} / \mathrm{Ca}$ - und $\mathrm{Sr} / \mathrm{Ca}-W e r t e$ getestet, um anschließend die beiden Korrelationskoeffizienten miteinander zu vergleichen (vgl. 2.3, S. 27f). Da der statistische Vergleich zweier Korrelationskoeffizienten für nichtparametrische Korrelationsmaße nicht möglich ist, wurde für diese Statistiken die Produkt-Moment-Korrelation verwendet. Der Vergleich der Korrelationen erfolgte hierbei über die transformierten Koeffizienten (Fishers ZTransformation). Sowohl für die Signifikanzprüfung der gruppeninternen Korrelationen, als auch für den Koeffizientenvergleich werden für die Werte der involvierten Variablen die allgemeinen Verteilungskriterien vorausgesetzt (vgl. Prüfung der Testvoraussetzungen, S. 67f). Zusätzlich zur Normalität der Datenwerte der Einzelvariablen wird eine bivariate Normalverteilung angenommen. Die Produkt-Moment-Korrelation gilt jedoch als äußerst robust gegenüber Verletzungen der Verteilungsvoraussetzungen. Eine Augenscheinprüfung hinsichtlich einer möglichst bivariaten Werteverteilung in Form einer Ellipse wird als ausreichend empfohlen (vgl. Bortz 1999, S. 205). Nach Shapiro-Wilk's W-Test signifikante Abweichungen von der Normalität der Werteverteilungen konnten daher vernachlässigt werden. Als Maß für eine ellipsoide Verteilungscharakteristik wurden in die bivariaten Darstellungen der Korrelationen die Konfidenzellipsen (engl.: prediction interval ellipse) mit einem Konfidenzkoeffizienten von 95\% eingezeichnet. Deren Berechnung anhand der Werteverteilung erfolgte mit STATISTICA (StatSoft 1998, zur Berechnungsgrundlage vgl. StatSoft 2001). Form und Lage dieser Ellipse sowie die Verteilung der Datenwerte in Bezug zu ihr charakterisieren die bivariate Werteverteilung, veranschaulichen die Güte des Zusammenhanges und erlauben zusätzlich die Abschätzung von Extremwerten. Wenn letztere weit vom Wertepool entfernt sind und damit den Korrelationskoeffizienten möglicherweise stark verfälschen, besteht durch diese Augenscheinprüfung die Möglichkeit, sie bei der Berechnung des Korrelationskoeffizienten auszuschließen (vgl. StatSoft 2001 zur allgemeinen Problematik eines solchen Vorgehens vgl. Ausreißer und Extremwerte, S. 66f).

Für die Korrelationsanalyse der $\mathrm{Ba} / \mathrm{Ca}$ - und $\mathrm{Sr} / \mathrm{Ca}-W e r t e$ der LA-ICP-TOFMS-Messungen innerhalb eines Kompaktaquerschnittes (vgl. 3.2.2.2, S. 56) wurde ebenfalls die Produkt-Moment-Korrelation verwendet. 


\section{Testvorgaben und Signifikanzniveau}

Alle Unterschiedshypothesen wurden von ungerichteten Hypothesen ausgehend zweiseitig statistisch getestet.

Die korrelationsanalytischen Prüfungen waren auf die Existenz hypothetisch positiver Zusammenhänge ausgerichtet und erfolgten daher einseitig. Die Vergleiche der Korrelationskoeffizienten wurden ebenfalls einseitig durchgeführt, da die Richtung des Unterschieds theoretisch vorgeben war (vgl. 2.3, S. 27).

Als Signifikanzniveau ist für alle Tests $5 \%(\boldsymbol{\alpha}=\mathbf{0 , 0 5})$ festgelegt worden. Die Überschreitungswahrscheinlichkeiten werden bis $\mathrm{p}<0,001$ abgestuft angegeben (kleinere Werte werden ebenfalls als $\mathrm{p}<0,001$ aufgeführt). Alle Aussagen beziehen sich auf das gewählte Signifikanzniveau. Es erfolgt keine weitere Differenzierung nach geringeren Niveaus.

Im Rahmen der Überprüfungen von Unterschiedshypothesen wurden teilweise zwei Gruppen mehrfach (jeweils einzeln für jede Variable) mit dem gleichen Verfahren statistisch verglichen. Für solche "simultanen" Signifikanztests ist der statistischen Logik folgend eine $\boldsymbol{\alpha}$-Fehler-Adjustierung erforderlich (vgl. Bortz et al. 2000, S. 48ff). Für diese Untersuchung wurde die Bonferroni-Korrektur verwendet. Der adjustierte $\boldsymbol{\alpha}^{*}$-Wert berechnet sich hierbei aus dem ursprünglichen $\boldsymbol{\alpha}$ und der Anzahl der "simultanen" Prüfungen innerhalb eines Hypothesenkontextes $\boldsymbol{k}$ mit $\boldsymbol{\alpha}^{*}=\boldsymbol{\alpha} / \boldsymbol{k}$. Wichtig ist der Hinweis auf den "Hypothesenkontext". Das bedeutet, daß nicht alle simultanen Vergleiche (hier: Anzahl der getesteten Variablen) in die Korrektur einfließen müssen, sondern nur jene die durch die a priori definierte Hypothesenstruktur verknüpft werden.

Für die Mehrfachvergleiche in dieser Untersuchung wurde fragestellungsorientiert eine Hypothesenstruktur mit zwei Variablengruppen respektive Hypothesenkontexten definiert.

Der erste Hypothesenkontext schließt die beiden Variablen $\mathrm{Sr} / \mathrm{Ca}$ und $\mathrm{Ba} / \mathrm{Ca}$ ein. Als die ursprünglichen Meßgrößen zur Nahrungsrekonstruktion sind diese sachlogisch gekoppelt (vgl. 1.1). Bei Gruppenvergleichen hinsichtlich beider Variablen wird $\boldsymbol{\alpha}$ für jeden der beiden Einzelvergleiche auf $\boldsymbol{\alpha}^{*}=\mathbf{0 , 0 5 / 2}=\mathbf{0 , 0 2 5}$ adjustiert, unabhängig davon, ob noch weitere Variablen getestet wurden.

In der zweiten Gruppe sind die Variablen zur Diagenesecharakterisierung zusammengefaßt (vgl. 2.3 und 2.4). Für diese Variablen ergeben sich aus der Fragestellung a priori Einzelhypothesen. Für jede dieser Variablen wird unabhängig von den anderen geprüft, ob es zwischen den durch unterschiedliche Erhaltungszustände des Knochens definierten Grup- 
pen einen Unterschied gibt. Es wird für jede Variable die Irrtumswahrscheinlichkeit von $5 \%$ akzeptiert. 


\section{Ergebnisse}

In den Ergebnissen werden alle relevanten Daten graphisch dargestellt. Jegliche Rohdaten sowie die jeweiligen Stichprobenkennwerte (Mittelwert, Standardabweichung usw.) sind ausschließlich im Anhang aufgelistet (S. 166ff). Statistische Testergebnisse werden im Ergebnisteil tabelliert aufgeführt. Die aus den Meßdaten und ihren statistischen Auswertungen abgeleiteten Ergebnisse bzw. Aussagen werden in entsprechenden Abbildungen veranschaulicht.

Die Daten der Referenzmaterialmessungen für die verschiedenen Analysemethoden sind ebenfalls nicht in den Ergebnisteil integriert, sondern im Anhang (Tabelle 21 und Tabelle 22) zusammengefaßt. Entsprechend diesen Daten wurde die Präzision für alle in den Auswertungen berücksichtigten Analysen als hinreichend hoch bewertet. Die Relevanz der Präzision bezieht sich auf die Tatsache, daß die vergleichenden Auswertungen der Meßdaten jeweils populationsintern (meßserienintern) erfolgten und daher die serieninterne Reproduzierbarkeit der Meßergebnisse (und damit die Vergleichbarkeit) im Vordergrund stand. Populationsübergreifende zusammenfassende Aussagen werden aus den Ergebnissen der serienintern durchgeführten statistischen Auswertungen abgeleitet, d.h. über Mustervergleiche. Direkte Meßdatenvergleiche erfolgten rein deskriptiv ohne statistische Prüfung mit entsprechend wenig Gewicht bei der Auswertung. Für diese Vergleiche wurde die Richtigkeit der Konzentrationsbestimmungen ebenfalls als ausreichend bewertet (vgl. Anhang, Tabelle 21 und Tabelle 22 mit den Referenzdaten in Tabelle 20). Größere Abweichungen sind nur für die kleine Stichprobe der Natriumbestimmungen mit der ICP-OES festzustellen. Für diese Analysen war aber ausschließlich der stichprobeninterne Vergleich relevant und damit nur die Reproduzierbarkeit von Bedeutung (vgl. 4.4.4).

\section{Ergebnisstruktur}

Die Gliederung der Ergebnisse folgt der Struktur der in Kapitel 2 dargelegten Konzeption (zusammengefaßt in 2.6). Die Ergebnisse werden damit der Chronologie der Fragestellungen und der sich aus den Teilergebnissen ableitenden Hypothesenentwicklung entsprechend aufgeführt. Daher sind teilweise von einem Ergebniskapitel zum nächsten überleitende und die Ergebnislogik erläuternde Argumentationen notwendig. Diese gehen teilweise über den Rahmen einer stringenten Ergebnisdarstellung hinaus. 


\subsection{Ergebnisse zur populationsinternen Variabilität von $\mathrm{Ba} / \mathrm{Ca}$ - und Sr/Ca-Werten}

\section{Ein Gruppenmuster der Ba/Ca- und Sr/Ca-Werte in der Skelettpopulation von Weingarten und die Varianzanalyse seiner möglichen Faktoren}

Ausgangspunkt der Auswertungen bilden die $\mathrm{Ba} / \mathrm{Ca}$ - und $\mathrm{Sr} / \mathrm{Ca}-W e r t e$ der Skelettserie von Weingarten. Den Ausführungen zur spezifischen Fragestellung für Weingarten entsprechend (vgl. 2.1, S. 19ff) wurden der histologischen Gruppeneinteilung (gut/schlecht) die biologische Gruppierungsvariante Geschlecht (männlich/weiblich) sowie die kulturhistorische des sozialen Ranges (niederer/hoher) gegenübergestellt. Es galt zu prüfen, ob und welche dieser drei Faktoren einen Einfluß auf die Elementkonzentrationen von Barium und Strontium innerhalb der Bevölkerung von Weingarten haben. Entsprechend dieser Hypothesenstruktur erfolgte die statistische Überprüfung durch eine Varianzanalyse (nichtparametrische ATS, vgl. 3.2.3.4, S. 69).

In Abbildung 5 sind die bivariaten Verteilungsmuster der $\mathrm{Ba} / \mathrm{Ca}-$ und $\mathrm{Sr} / \mathrm{Ca}-W e r t e$ jeweils nach den zu vergleichenden Gruppierungsfaktoren kategorisiert dargestellt (zum dargestellten und gegenüber der früheren Untersuchung reduzierten Stichprobenumfang infolge Probenausschluß vgl. 2.1, S. 13 und 3.1.1, S. 39). Es wird für die drei Faktoren der differentielle Grad der "Entmischung" der Gruppen veranschaulicht. Hiernach ist für die Geschlechtergruppen eine Differenzierung nicht erkennbar. Für die Sozialstatusgruppen sind die Individuen mit hohem sozialen Rang augenscheinlich gleich verteilt, bei jenen mit niederem Rang scheinen aber höhere Konzentrationswerte vorzuherrschen. Nach dieser Darstellung sind die histologischen Gruppen am deutlichsten separiert.

Die Ergebnisse der für beide Konzentrationsquotienten separat durchgeführten Varianzanalyse sind in Tabelle 1 aufgelistet. Diesen statistischen Prüfungen zufolge gibt es nur zwischen den beiden histologischen Gruppen einen Unterschied (für beide Variablen $\mathrm{p}<0,001)$. Knochenproben mit schlechtem histologischen Erhaltungszustand unterscheiden sich hinsichtlich der $\mathrm{Ba} / \mathrm{Ca}$ - und $\mathrm{Sr} / \mathrm{Ca}-W e r t e$ von Knochenproben mit gutem histologischen Erhaltungszustand (vgl. Abbildung 5c). Alle anderen Gruppenkonstrukte zeigen keine Unterschiede. Für beide Elementkonzentrationen ist der im Hinblick auf frühere spezifische Auswertungsergebnisse geprüfte Einfluß der Faktorinteraktion GeschlechtSozialstatus (vgl. 2.1, S. 20) statistisch ebenfalls nicht abzusichern.

Als Konsequenz dieser Ergebnisse sind weitere Auswertungen hinsichtlich der biologischen und kulturellen Faktoren für die Knochenproben von Weingarten hinfällig. Es galt 
nun zu prüfen, ob der für Weingarten statistisch wahrscheinliche Einfluß der Histologie auf die Barium- und Strontiumgehalte der Knochenproben auch in weiteren Skelettpopulationen (Kirchheim unter Teck und Wenigumstadt) vorhanden ist. 


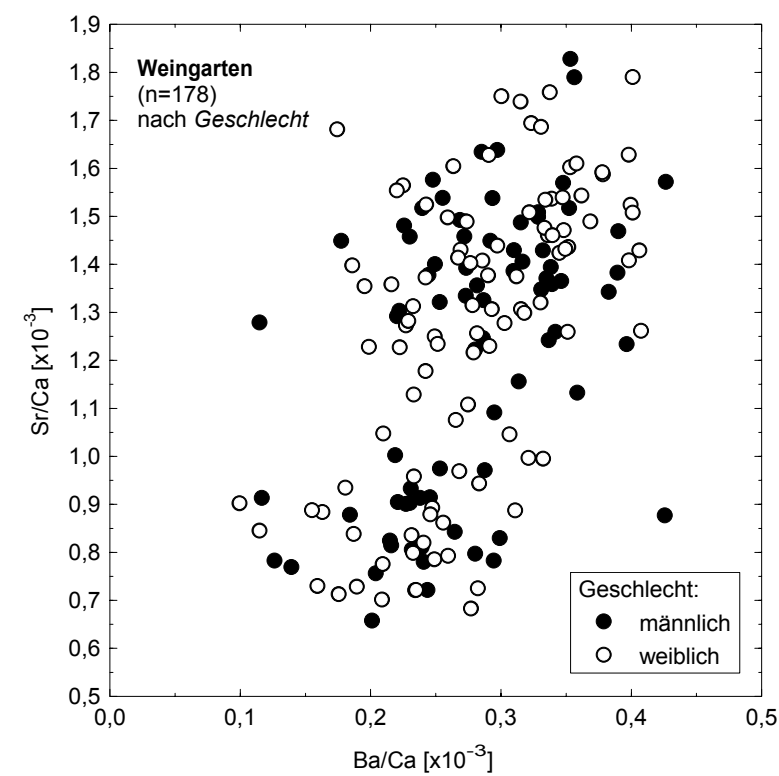

a)

Abbildung 5: Mögliche Gruppierungsfaktoren für die $\mathrm{Ba} / \mathrm{Ca}$ - und $\mathrm{Sr} / \mathrm{Ca}-$ Werte der Individuen von Weingarten

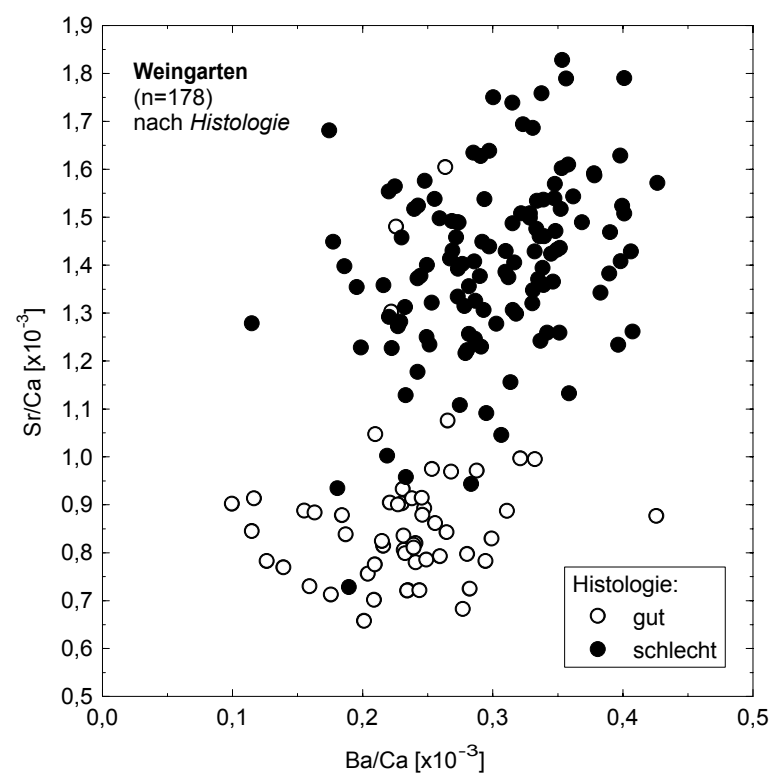

c)

Die Scatterplots sind zum Vergleich nach a) Geschlecht (männlich/weiblich), b) Sozialstatus (niederer/hoher Rang), und c) Histologie (gut/schlecht) kategorisiert.

Tabelle 1: Ergebnisse für die drei möglichen Gruppierungsfaktoren der $\mathrm{Ba} / \mathrm{Ca}$ - und $\mathrm{Sr} / \mathrm{Ca}-$ Werte von Weingarten (nichtparametrische Varianzanalyse).

\begin{tabular}{|c|c|c|c|c|c|c|c|c|}
\hline \multirow{3}{*}{\begin{tabular}{|l} 
Faktor / Interaktion \\
Geschlecht \\
Sozialstatus \\
Histologie \\
Geschlecht - Sozialstatus
\end{tabular}} & \multirow{2}{*}{\multicolumn{4}{|c|}{ Gruppengrößen }} & \multicolumn{2}{|c|}{$\mathrm{Ba} / \mathrm{Ca}$} & \multicolumn{2}{|c|}{$\mathrm{Sr} / \mathrm{Ca}$} \\
\hline & & & & & F-Wert & p-Wert & F-Wert & p-Wert \\
\hline & $\begin{array}{l}\text { weiblich } \\
\text { niederer Rang } \\
\text { gut }\end{array}$ & $\begin{array}{l}=78 \\
=95 \\
=56\end{array}$ & $\begin{array}{l}\text { männlich } \\
\text { hoher Rang } \\
\text { schlecht }\end{array}$ & $\begin{array}{l}=100 \\
=83 \\
=122\end{array}$ & $\begin{array}{r}0,25 \\
0,28 \\
64,05 \\
0,04\end{array}$ & $\begin{array}{l}0,618 \\
0,6 \\
<0,001^{+} \\
0,834\end{array}$ & $\begin{array}{r}0,11 \\
0,17 \\
198,98 \\
0,75\end{array}$ & $\begin{array}{r}0,744 \\
, 684 \\
<0,001^{+} \\
0,392\end{array}$ \\
\hline
\end{tabular}

$\boldsymbol{\alpha}=\mathbf{0 , 0 5}$ mit Bonferroni-Korrektur $\boldsymbol{\alpha}^{*}=\mathbf{0 , 0 2 5}$ (vgl. 3.2.3.4, S. 72f)

${ }^{+}$bei festgelegtem $\alpha^{*}$ signifikant 
Histologischer Erhaltungszustand und Konzentrationsgruppen in den Populationen von Kirchheim unter Teck und Wenigumstadt

Auf der Grundlage der Ergebnisse von Weingarten wurden für Kirchheim unter Teck und Wenigumstadt die statistischen Prüfungen auf die Vergleiche der Histologiegruppen beschränkt. Dies bedeutet, die Prüfung des Einflusses des histologischen Erhaltungszustandes auf die $\mathrm{Ba} / \mathrm{Ca}$ - und $\mathrm{Sr} / \mathrm{Ca}$-Werte wurde ohne Gegenüberstellung mit anderen Faktoren durchgeführt. Diese Vorgehensweise begründet sich unabhängig von den Weingarten-Ergebnissen zusätzlich aus den früheren Auswertungen dieser Serien (vgl. 2.1, S. 21f).

In Abbildung 6 sind zunächst die Verteilungen der $\mathrm{Ba} / \mathrm{Ca}$ - und $\mathrm{Sr} / \mathrm{Ca}-W e r t e$ von Kirchheim unter Teck und Wenigumstadt (zum Stichprobenumfang vgl. 2.1, S. 13 und 3.1.1, S. 39f) im Vergleich zu Weingarten dargestellt. Hierbei wird deutlich, daß, im Gegensatz zu Weingarten mit seiner erkennbaren dichotomen Verteilung, Kirchheim unter Teck und Wenigumstadt durch eine kontinuierlichere Werteverteilung charakterisiert sind. Es galt statistisch zu prüfen, ob sich trotz der kontinuierlichen Gesamtverteilungen innerhalb dieser beiden Skelettpopulationen die histologischen Gruppen über Konzentrationsunterschiede differenzieren. Die hierauf reduzierte Fragestellung konnte für jede der beiden Populationen durch einen einfachen Zweigruppenvergleich (Mann-Whitney-U-Test, vgl. 3.2.3.4, S. 70) zwischen den histologisch gut erhaltenen Proben und den histologisch schlecht erhaltenen Proben erfolgen. Die Ergebnisse hierzu sind in Tabelle 2 aufgeführt. Hiernach gibt es sowohl für Kirchheim unter Teck als auch für Wenigumstadt Unterschiede zwischen den Histologiegruppen hinsichtlich der $\mathrm{Ba} / \mathrm{Ca}-$ und $\mathrm{Sr} / \mathrm{Ca}-\mathrm{Werte}$. 


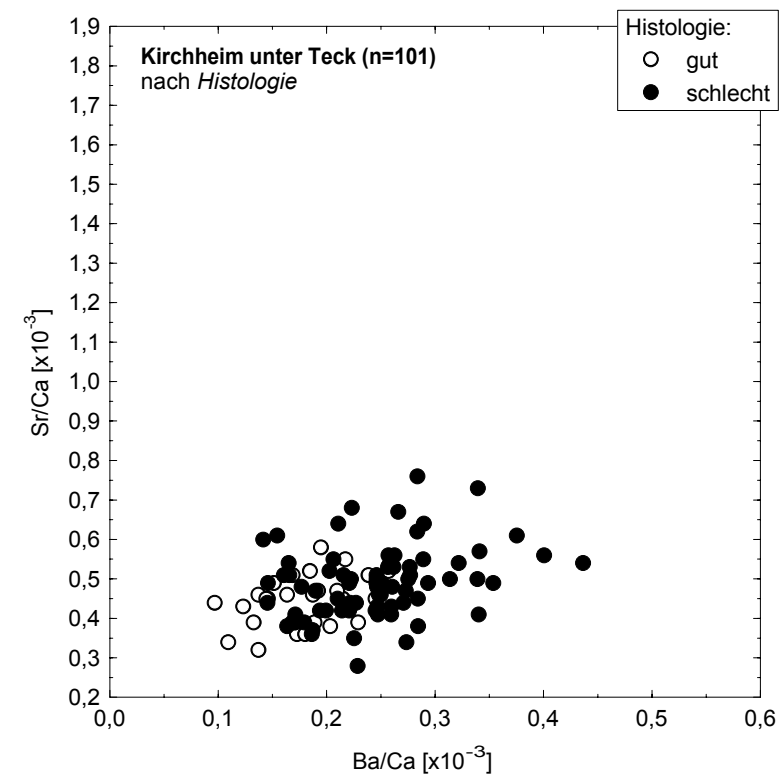

a)

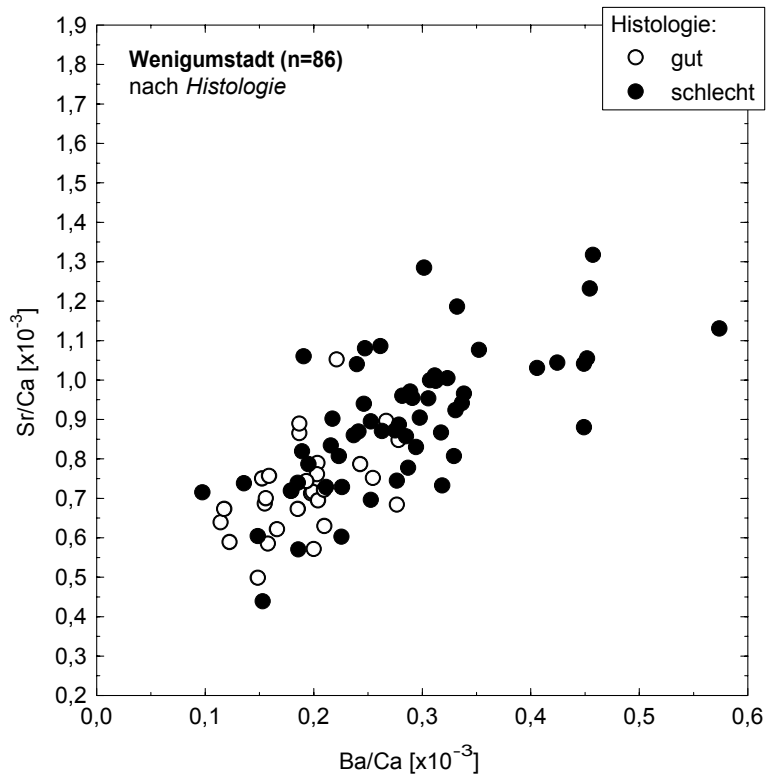

b)

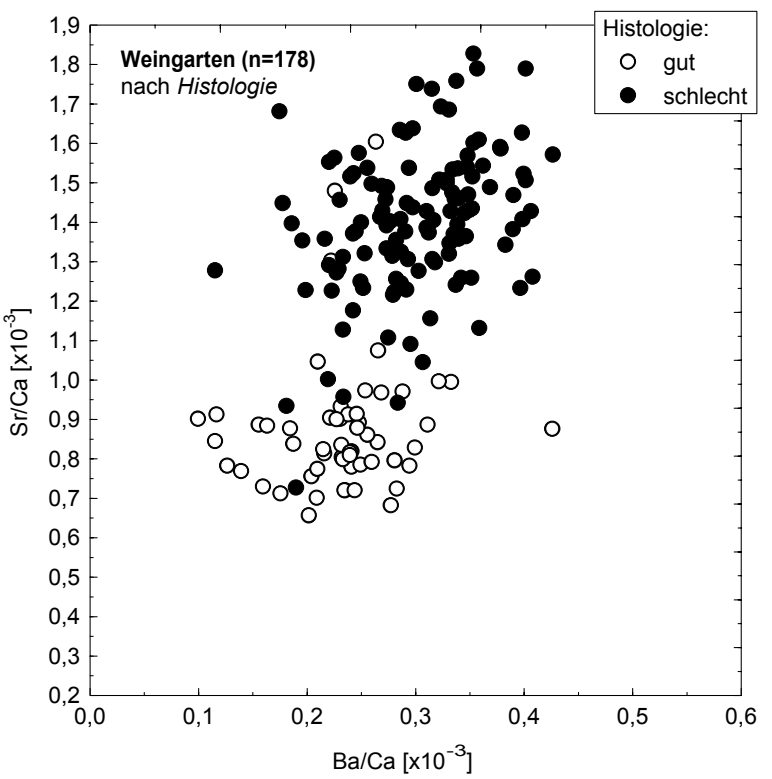

c)

Abbildung 6: Vergleich der diagenetischen Gruppenmuster der Ba/Ca- und Sr/Ca-Werte zwischen a) Kirchheim unter Teck, b) Wenigumstadt und c) Weingarten.

Die Scatterplots sind nach Histologie (guter Erhaltungszustand vs. schlechter Erhaltungszustand) kategorisiert.

Tabelle 2: Statistische Vergleiche (Mann-Whitney-U-Test) der histologischen Gruppen von Kirchheim unter Teck und Wenigumstadt hinsichtlich Unterschieden der $\mathrm{Ba} / \mathrm{Ca}$ - und $\mathrm{Sr} / \mathrm{Ca}-W e r t e$.

\begin{tabular}{|l|c|c|c|c|c|c|}
\hline \multirow{2}{*}{ Population } & \multicolumn{2}{|c|}{ Gruppengrößen } & \multicolumn{2}{c|}{ Ba/Ca } & \multicolumn{2}{c|}{ Sr/Ca } \\
\cline { 2 - 7 } & gute Histologie & schlechte Histologie & Z-Wert & p-Wert & Z-Wert & p-Wert \\
\hline Kirchheim unter Teck & 28 & 73 & $-5,12$ & $<0,001^{+}$ & $-2,56$ & $0,01^{+}$ \\
Wenigumstadt & 29 & 57 & $-5,03$ & $<0,001^{+}$ & $-4,73$ & $<0,001^{+}$ \\
\hline
\end{tabular}

$\boldsymbol{\alpha}=\mathbf{0 , 0 5}$ mit Bonferroni-Korrektur $\boldsymbol{\alpha}^{*}=\mathbf{0 , 0 2 5}(\mathrm{vgl}$. 3.2.3.4, S. 72f)

${ }^{+}$bei festgelegtem $\boldsymbol{\alpha}^{*}$ signifikant 


\section{Histologischer Erhaltungszustand und ein systematisches Gruppenmuster}

Für die drei Populationen Weingarten, Kirchheim unter Teck und Wenigumstadt sind folgende Ergebnisaspekte zusammenzufassen:

- Die über den histologischen Erhaltungszustand des Knochenmaterials definierten Gruppen von Knochenproben unterscheiden sich populationsintern hinsichtlich ihrer $\mathrm{Ba} / \mathrm{Ca}-$ und $\mathrm{Sr} / \mathrm{Ca}-\mathrm{Werte}$.

- Diese Unterschiede der Konzentrationsverhältnisse zwischen den Histologiegruppen sind systematisch. In allen drei Populationen zeichnen sich die Knochenproben mit schlechter Histologie gegenüber den Knochenprobe mit guter Histologie durch höhere $\mathrm{Ba} / \mathrm{Ca}-\mathrm{bzw}$. Sr/Ca-Werte aus.

In Abbildung 7 sind die statistisch deskriptiven Daten ${ }^{3}$ der Gruppen (Mittelwert, Standardabweichung und Spannweite) für diese Vergleiche dargestellt.

\footnotetext{
${ }^{3}$ Zur Darstellungsform deskriptiv statistischer Kenngrößen ein für die weitere Ergebnisdokumentation generell gültiger Hinweis:

In 3.2.3.4 (S. 68) ist darauf hingewiesen worden, daß die Werteverteilungen verschiedener Variablen innerhalb untersuchter Datengruppen teilweise nicht der Normalverteilung entsprechen. Da diese Abweichungen hinsichtlich der Charakterisierung der zentralen Tendenz der Gruppen aber nicht gravierend waren, wurde sich generell für die Darstellung der Kenngrößen einer normalverteilten Stichprobe (Mittelwert und Standardabweichung) entschieden. Die Darstellung von statistisch definierten Ausreißern und Extremwerten ermöglicht eine Abschätzung deren Bedeutung für die Verteilungscharakteristik (vgl. 3.2.3.4, S. 66f). Durch die zusätzliche Darstellung der Spannweite ist in Kombination mit Lage und Relation der anderen Kenngrößen die jeweilige Datengruppe hinreichend charakterisiert.
} 

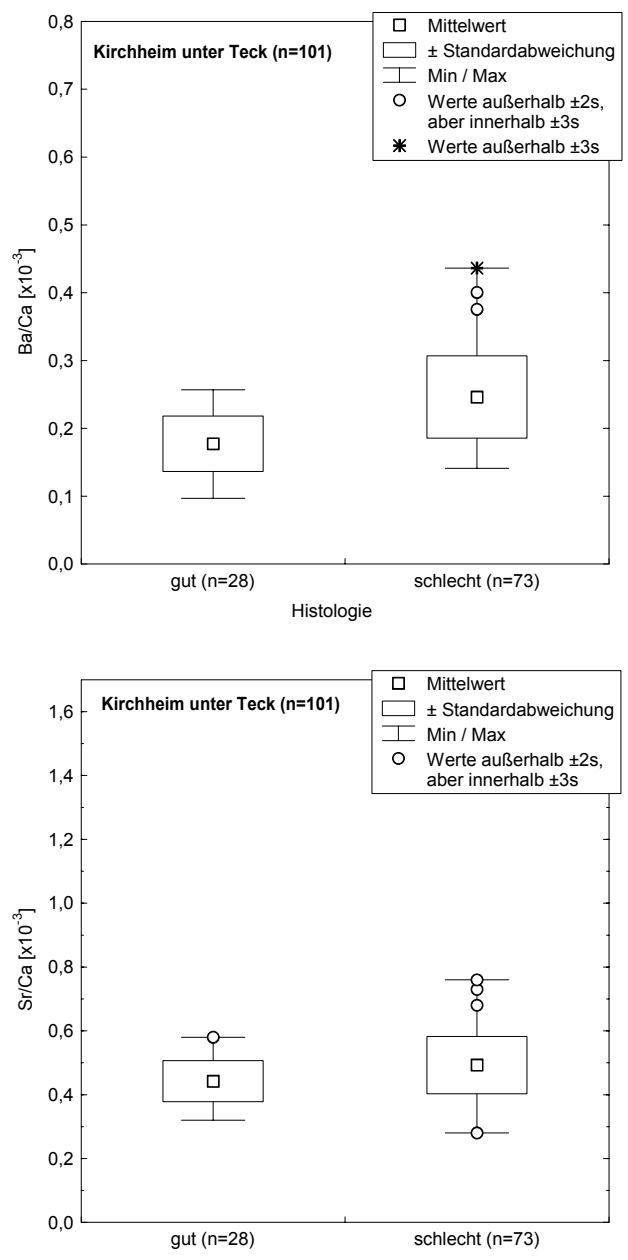
und Weingarten $(\mathrm{c}+\mathrm{f})$.

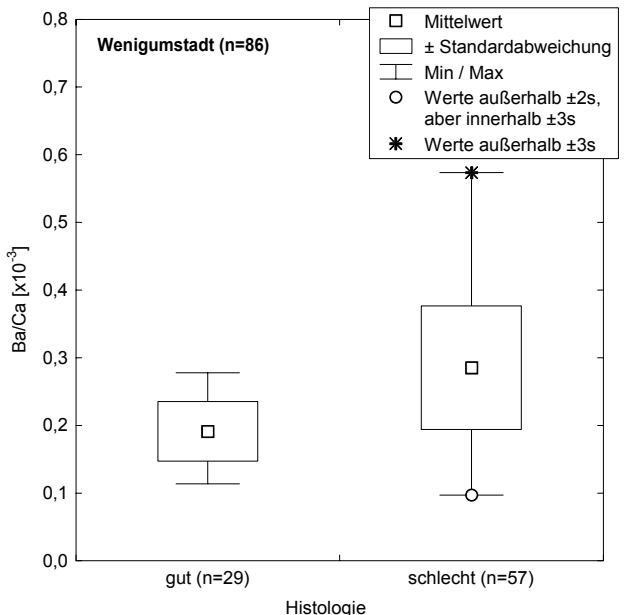

b)

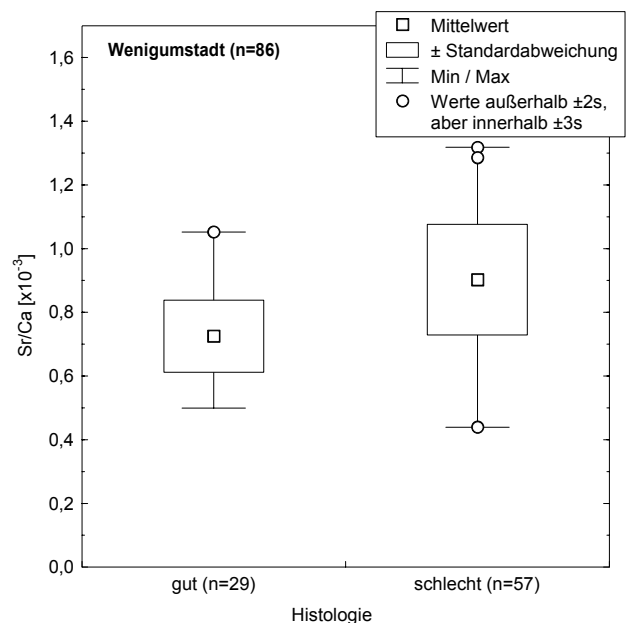

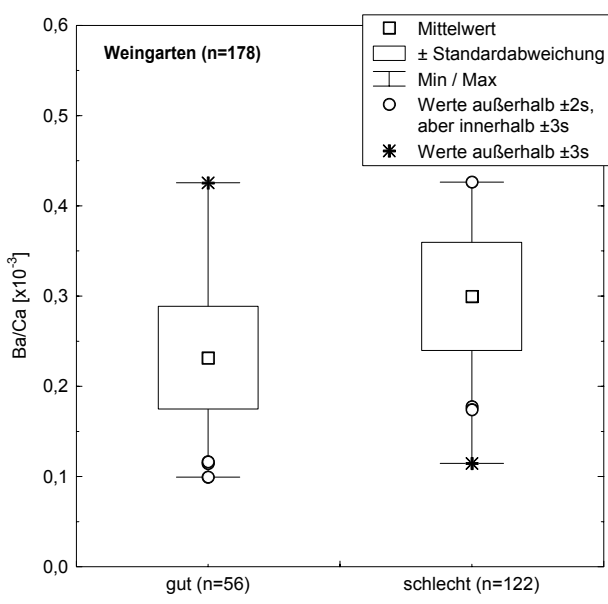

c)

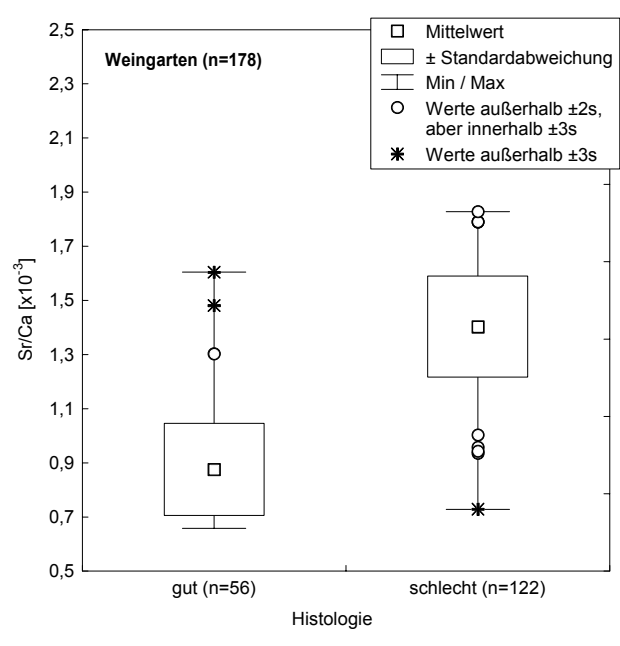

f) 


\subsection{Ergebnisse der Untersuchungen zur Probenaufreinigung}

Die Ergebnisse des vorangegangen Kapitels resultieren aus Konzentrationsanalysen an Knochenproben, die alle chemisch aufgereinigt waren (vgl. 3.2.1.3). Für diese Proben standen keine Vergleichsdaten unbehandelter Pendants zur Verfügung.

In diesem Ergebnisteil werden daher zwei Teilaspekte erfaßt. Zunächst wird anhand von Konzentrationen nicht aufgereinigter Knochenproben die Frage beantwortet, ob die beobachteten histologieabhängigen Konzentrationsmuster bodengelagertem Knochenmaterial primär eigen sind. Durch den anschließenden Vergleich der Konzentrationen der Knochenproben vor und nach der Probenaufreinigung wird zusätzlich der Einfluß der Probenaufreinigung spezifiziert (vgl. 2.2).

\section{Histologischer Erhaltungszustand und Konzentrationsgruppen in der Population von Kirchheim am Ries vor und nach der Probenaufreinigung}

Dem ersten Teil der Problemstellung entsprechend bilden Knochenkonzentrationen nicht aufgereinigter Proben beider histologischer Erhaltungsgruppen den Ausgangspunkt der Auswertungen. In Abbildung 8a sind für die entsprechenden Stichproben der Skelettserie von Kirchheim am Ries die bivariaten Verteilungen der $\mathrm{Ba} / \mathrm{Ca}-$ und $\mathrm{Sr} / \mathrm{Ca}-W e r t e$ dargestellt. Für unbehandelte Knochenproben ist hier das von den vorangegangen Ergebnissen bekannte Verteilungsmuster mit höheren Werten für histologisch schlecht erhaltene Proben gegenüber den histologisch gut erhalten Proben wiederzufinden.

In den weiteren Abbildung 8b-d werden von den gleichen Knochenproben die $\mathrm{Ba} / \mathrm{Ca}-$ und $\mathrm{Sr} / \mathrm{Ca}-W e r t e$ nach verschiedenen Methoden der Probenaufreinigung gezeigt. Diese Gegenüberstellung gestattet über einen einfachen Mustervergleich per Augenschein die Werteverteilungen vor und nach der Vorbehandlung einzuschätzen. Es kann auf dieser Auswertungsebene festgestellt werden, daß die Charakteristik des ursprünglichen Musters der unbehandelten Knochenproben auch nach chemischer Aufreinigung oder mechanischer Oberflächenbehandlung bzw. nach der Kombination beider Varianten erhalten bleibt. 

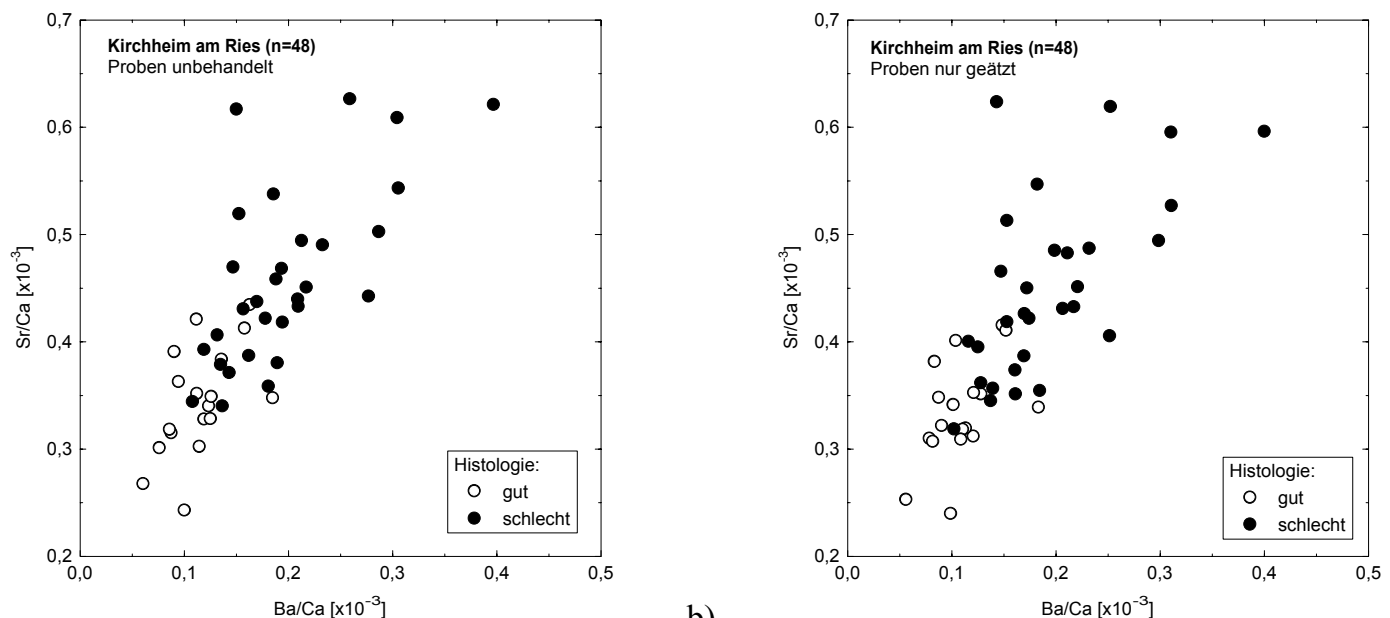

a)

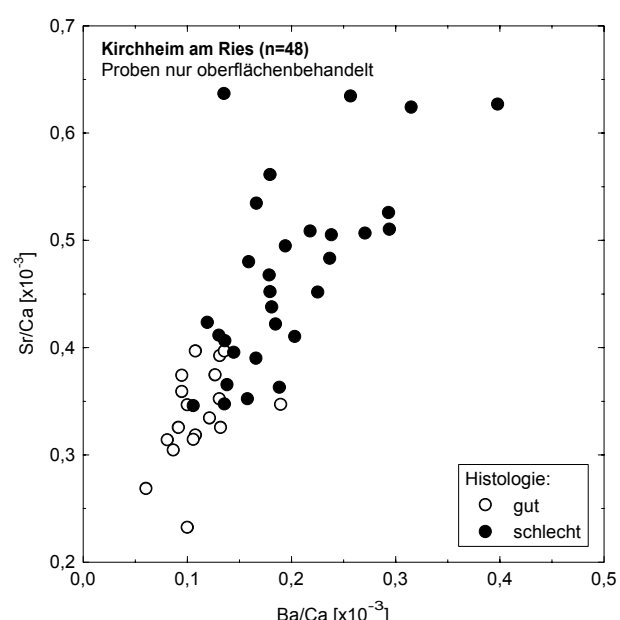

b)

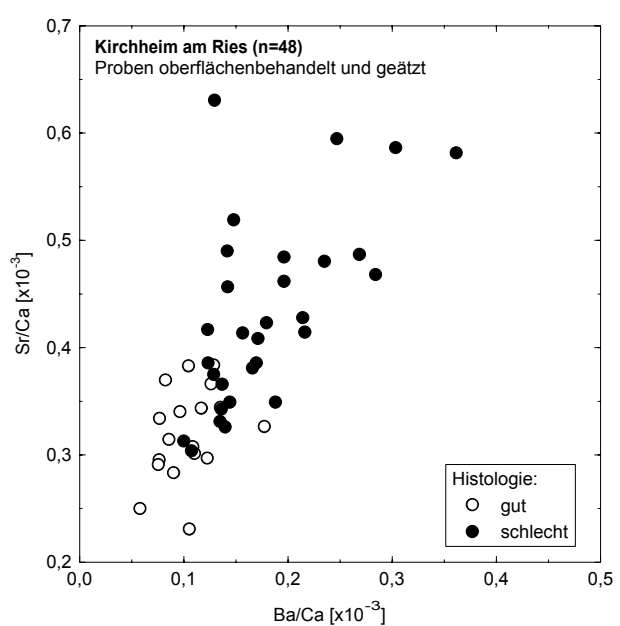

c)

Abbildung 8: Das Gruppenmuster (gute vs. schlechte Histologie) der Ba/Ca- und Sr/Ca-Werte von Kirchheim am Ries.

Das Muster ist in Abhängigkeit von den verschiedenen Methoden der Probenaufreinigung, a) unbehandelt, b) nur geätzt, c) nur oberflächenbehandelt und d) oberflächenbehandelt mit anschließendem Ätzen dargestellt.

Diese Beobachtung eines persistierenden Musters der $\mathrm{Ba} / \mathrm{Ca}$ - und $\mathrm{Sr} / \mathrm{Ca}-W e r t e$ wird gestützt durch den für beide Variablen getrennt dargestellten statistisch deskriptiven Vergleich in Abbildung 9. Zwischen den histologischen Gruppen ist, unabhängig davon, ob und wie die Proben vorbehandelt wurden, generell ein Unterschied wahrscheinlich. Ebenso unabhängig von der Probenaufreinigung bleiben jeweils die Gruppenmittelwerte der gut erhaltenen Proben sowie die der schlecht erhaltenen annähernd auf ihrem Niveau. 
a)

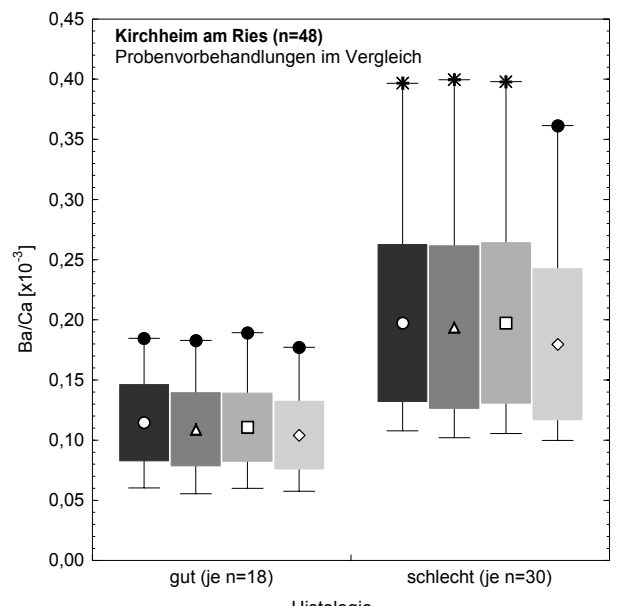

b)

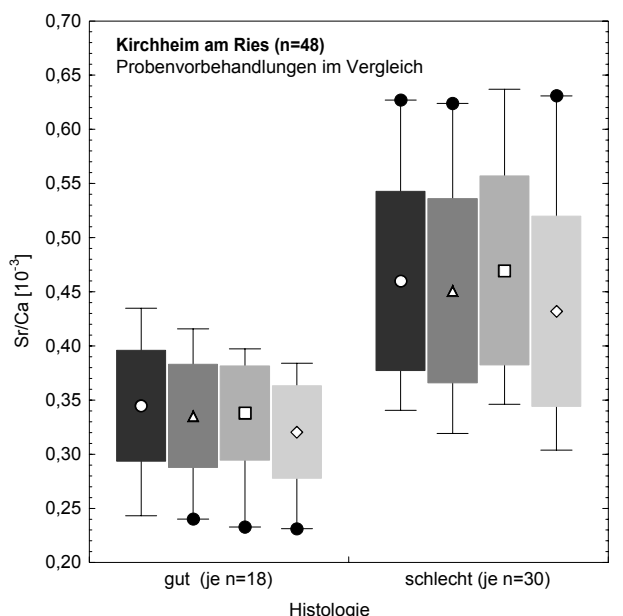

Abbildung 9: Statistisch deskriptiver Vergleich von a) Ba/CaWerten und b) $\mathrm{Sr} / \mathrm{Ca}-W e r t e n$ zwischen den Histologiegruppen von Kirchheim am Ries.

Durch die in der linksstehenden Legende den Mittelwerten zugeordneten Symbole und den dazugehörigen Schattierungen der Boxen werden die beiden diagenetischen Gruppen für die verschiedenen Aufreinigungsvarianten jeweils getrennt dargestellt.

\section{Aufreinigungsbedingte Veränderungen der Barium- und Strontiumkonzentrationen im detaillierten Vergleich}

Die differenzierte Auswertung der Elementkonzentrationen vor und nach der Probenaufreinigung führt zum zweiten Aspekt dieses Ergebnisteils. Unabhängig von einem scheinbar unzureichenden Effekt der Aufreinigung wird deren differentielle, von der Histologie der Proben abhängige, Auswirkung auf die $\mathrm{Ba} / \mathrm{Ca}$ - und $\mathrm{Sr} / \mathrm{Ca}-W e r t e$ geprüft. Die Hypothese lautet, daß die Konzentrationen der gut erhaltenen Knochenproben durch die Aufreinigung anders beeinflußt werden sollten als die der schlecht erhaltenen (vgl. 2.2). Die statistische Prüfung hinsichtlich Konzentrationsunterschieden zwischen den Histologiegruppen sowie deren Veränderung nach den Aufreinigungsmethoden erfolgte in diesem Kontext als Auswertung eines Meßwiederholungsdesigns (vgl. 3.2.3.4, S. 69). Die Ergebnisse der für $\mathrm{Ba} / \mathrm{Ca}$ bzw. Sr/Ca separat durchgeführten Varianzanalyse sind in Tabelle 3 aufgelistet. 
Tabelle 3: Statistische Überprüfungen für die Ba/Ca- und $\mathrm{Sr} / \mathrm{Ca}$-Werte von Kirchheim am Ries.

Durch nichtparametrische Varianzanalyse mit Meßwiederholung werden die Histologiegruppen verglichen, der Einfluß der Aufreinigungsmethoden überprüft und die histologiespezifische Aufreinigungswirkung getestet (Interaktion Histologie-Aufreinigung).

\begin{tabular}{|l|c|c|c|c|}
\hline \multirow{2}{*}{ Faktor / Interaktion } & \multicolumn{2}{|c|}{ Ba/Ca } & \multicolumn{2}{c|}{ Sr/Ca } \\
\cline { 2 - 5 } & B-Wert & p-Wert & B-Wert & p-Wert \\
\hline Histologie [gut = 18; schlecht = 30] & 52,85 & $<0,001^{+}$ & 47,63 & $<0,001^{+}$ \\
Aufreinigung [Methoden = 3+1(unbehandelt)] & 11,7 & $<0,001^{+}$ & 31,8 & $<0,001^{+}$ \\
Histologie - Aufreinigung & 0,61 & 0,534 & 1,83 & 0,164 \\
\hline
\end{tabular}

$\boldsymbol{\alpha}=\mathbf{0 , 0 5}$ mit Bonferroni-Korrektur $\boldsymbol{\alpha}^{*}=\mathbf{0 , 0 2 5}$ (vgl. 3.2.3.4, S. 72f)

${ }^{+}$bei festgelegtem $\boldsymbol{\alpha}^{*}$ signifikant

Als erstes Teilergebnis dieser Varianzanalyse wird für beide Variablen ein globaler Unterschied zwischen den Histologiegruppen erfaßt $(\mathrm{p}<0,001)$. Ein solcher Unterschied ist damit nicht einzeln für jeden Vergleich der Histologiegruppen für die verschiedenen Aufreinigungsmethoden bzw. für die unbehandelten Proben statistisch abgesichert. In Anbetracht des persistierenden Konzentrationsmusters (vgl. Abbildung 8) wurde aber auf entsprechende Einzelvergleiche verzichtet.

Das zweite Teilergebnis bedeutet, daß die $\mathrm{Ba} / \mathrm{Ca}-$ und $\mathrm{Sr} / \mathrm{Ca}-$ Werte durch die verschiedenen Aufreinigungsmethoden verändert werden (statistisch abgesichert mit $p<0,001$ ). Einzelvergleiche zwischen dem unbehandelten Probensatz und den Aufreinigungsmethoden werden mit der Beobachtung nur marginaler Veränderungen (und damit mit derselben Begründung, wie für den Verzicht auf die Einzelvergleiche der Histologiegruppen, s.o.) wiederum als irrelevant eingeschätzt und nicht durchgeführt.

Im Hinblick auf die Fragestellung sind die Resultate der Varianzanalyse zur Interaktion zwischen der Histologie und der Aufreinigung entscheidend. Für keine der beiden Variablen konnten zwischen den Histologiegruppen Unterschiede bei den Veränderungen der Werte festgestellt werden. Die durch die Aufreinigungsmethoden bedingten Konzentrationsänderungen sind hiernach unabhängig vom histologischen Erhaltungszustand der Knochenproben.

Diese Feststellung wird in den Grafiken der Abbildung 10 anhand der Konzentrationsveränderungen der Einzelproben veranschaulicht. Es sind zwischen den beiden Histologiegruppen weder in der Gerichtetheit noch bei den Beträgen der Veränderungen systematische Unterschiede zu erkennen. 
a)
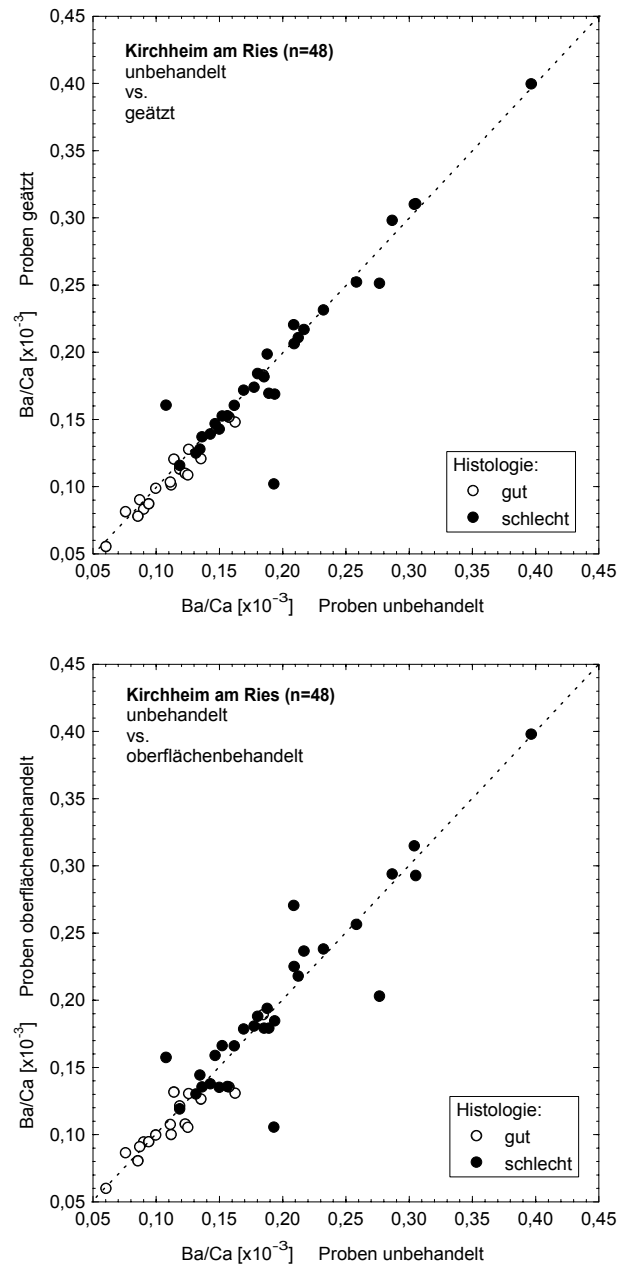

c)

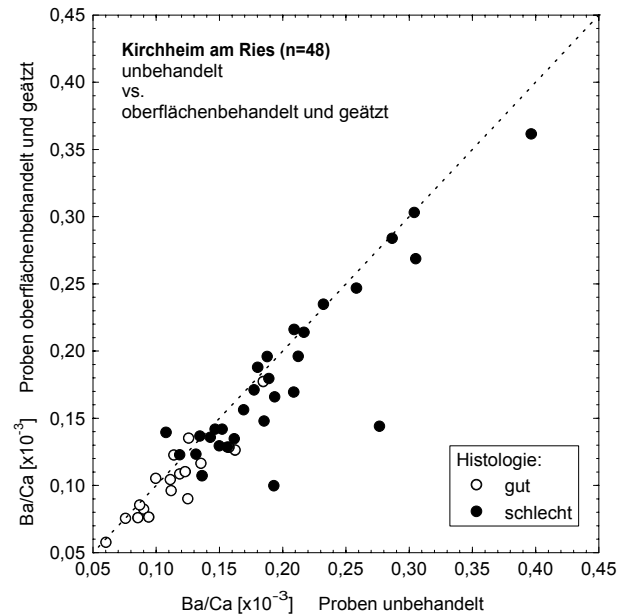

e)

Abbildung 10: Aufreinigungsbedingte Veränderungen der Ba/Ca-Werte (a, c, e) und $\mathrm{Sr} / \mathrm{Ca}-\mathrm{Werte}(\mathrm{b}, \mathrm{d}, \mathrm{f})$ für die Knochenproben von Kirchheim am Ries.

In a), b), c), d) und f) sind getrennt nach $\mathrm{Ba} / \mathrm{Ca}$ - und $\mathrm{Sr} / \mathrm{Ca}-W e r t e n$ jeweils die Daten der unbehandelten Proben gegen die Daten einer der Aufreinigungsmethoden aufgetragen. Damit erfolgt die Darstellung der Konzentrationsänderungen der Einzelproben über die vertikalen Abweichungen ihrer Datenpunkte von der Grafikdiagonalen. Die gestrichelte Diagonale markiert die "Neutrale". Alle Proben mit Werten auf dieser Geraden sind durch die jeweilige Probenvorbehandlung nicht beeinflußt worden. 


\section{Zusammenfassung der Ergebnisse der Untersuchungen zur Probenaufreinigung}

Für die vergleichenden Untersuchungen zur Probenaufreinigung können abschließend drei Aspekte zusammengefaßt werden:

- Auch bei nicht aufgereinigten Knochen sind Proben mit schlechter histologischer Erhaltung gegenüber gut erhaltenen durch höhere $\mathrm{Ba} / \mathrm{Ca}$ - und $\mathrm{Sr} / \mathrm{Ca}-W e r t e$ gekennzeichnet.

- Chemische, mechanische und aus beiden kombinierte Probenaufreinigung hat nur marginale und hinsichtlich der Histologiegruppen indifferente Konzentrationsveränderungen zur Folge.

- Entsprechend bleibt das primäre von den Histologiegruppen abhängige Konzentrationsmuster auch nach der Probenaufreinigung erhalten.

Von diesen Feststellungen bildet die Beobachtung bzw. Wiederfindung des histologieabhängigen Musters für die $\mathrm{Ba} / \mathrm{Ca}$ - und $\mathrm{Sr} / \mathrm{Ca}-W e r t e ~ d e n ~ A u s g a n g s p u n k t$ für die weiteren Auswertungen.

\subsection{Ergebnisse zur Prüfung diageneseanzeigender Kontrollkriterien}

Aus den bisherigen populationsinternen Vergleichen der beiden Histologiegruppen (gut/schlecht) ergibt sich folgende Schlußfolgerung: Die beiden ursprünglich ausschließlich über ihr knochenhistologisches Erscheinungsbild definierten Gruppen zeigen hinsichtlich der Elementkonzentrationen von Barium und Strontium systematische Unterschiede.

Diese chemische Entsprechung histologischer Unterschiede wird für die weiteren Auswertungen als diagenetisches Korrelat verstanden. Hiernach sind histologisch gut und schlecht erhaltene Knochenproben in unterschiedlichem Maße diagenetisch chemisch verändert.

Ausgehend von dieser Hypothese sollten jene Kriterien, die zur Charakterisierung chemischer Veränderungen der Knochensubstanz unter der Liegezeit verwendet werden sollen, zwischen den beiden Histologiegruppen ebenfalls differenzieren (Ba-Sr-Zusammenhang, $\mathrm{Ca} / \mathrm{P}-$ Verhältnis, Magnesium- und Aluminiumkonzentration sowie Masseverlust während der chemischen Probenaufreinigung bzw. während des Veraschens, vgl. 2.3). Daher werden die histologisch definierten Gruppen der vier in den vorangegangenen Ergebniskapi- 
teln untersuchten Skelettpopulationen (Weingarten, Kirchheim unter Teck, Wenigumstadt und Kirchheim am Ries) populationsintern hinsichtlich verschiedener diagenesecharakterisierender Kontrollkriterien verglichen.

Es wird geprüft, ob die verwendeten Kriterien die diagenetischen Gruppierungen unterscheiden. Hierbei wird das Differenzierungsvermögen jeder Kontrollvariablen einzeln getestet. Daher werden die entsprechenden "simultanen" Vergleiche eines Gruppenpaars mit statistisch unabhängigen Hypothesen durchgeführt. Es wird für jede Variable eine "eigene" Irrtumswahrscheinlichkeit von 5\% akzeptiert (vgl. 3.2.3.4, S. 72f).

\section{Ba-Sr-Zusammenhang}

Die in 2.3 (S. 27) erläuterte Hypothese zum Strontium-Barium-Zusammenhang innerhalb einer Bevölkerung und ihre Übertragung auf den populationsinternen Vergleich zweier Histologiegruppen mit unterschiedlich starker diagenetischer Veränderung der Knochensubstanz wird in diesem Ergebnispunkt ausgewertet. Entsprechend werden die gruppeninternen Korrelationen beider Histologiegruppen innerhalb der verschiedenen Populationen miteinander verglichen.

In Abbildung 11 sind für einen grafischen Vergleich die jeweiligen Zusammenhänge für Kirchheim unter Teck, Wenigumstadt und Weingarten anhand der standardisierten (z-transformierten) Datenwerte dargestellt. Abbildung 12 enthält den entsprechenden Vergleich der Gruppenkorrelationen für Kirchheim am Ries. Hier sind die Datenwerte der Knochenproben ohne Probenaufreinigung, also mit der theoretisch größten diagenetischen Divergenz, aufgetragen.

Die standardisierte Darstellung in beiden Abbildungen erlaubt sowohl den populationsinternen als auch Interpopulationsvergleiche. Ein solcher "orthogonaler" Vergleich zeigt mit Ausnahme von Weingarten bereits ohne Auswertung der Korrelationsanalysen eher populationsspezifische denn populationsintern histologiespezifische Charakteristika der Zusammenhänge. 


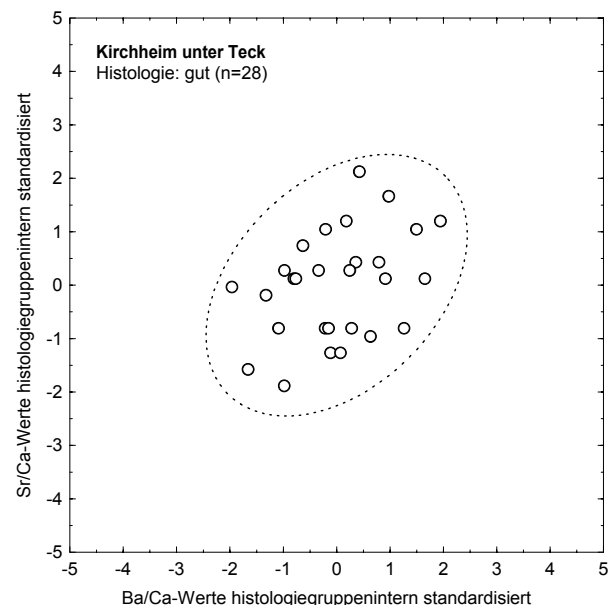

a)

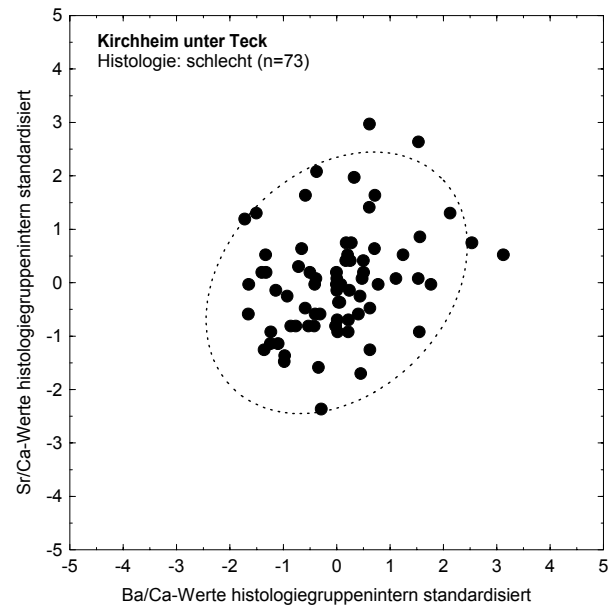

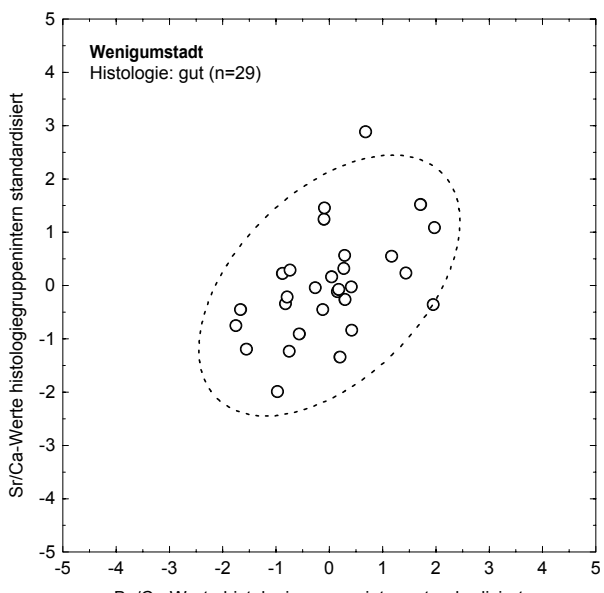

b)

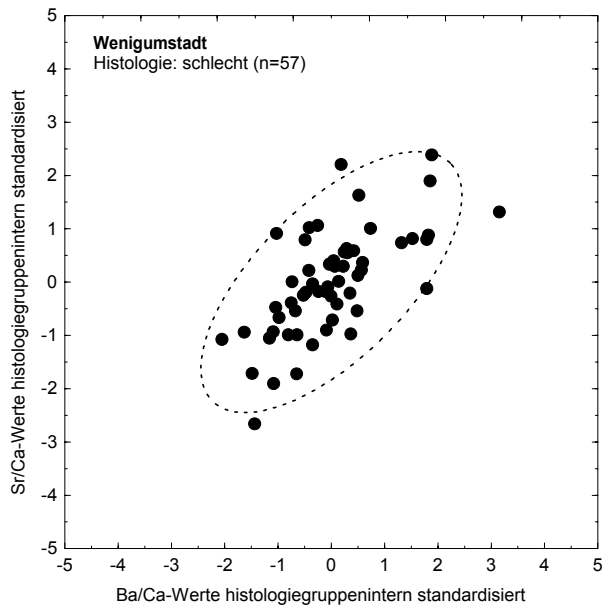

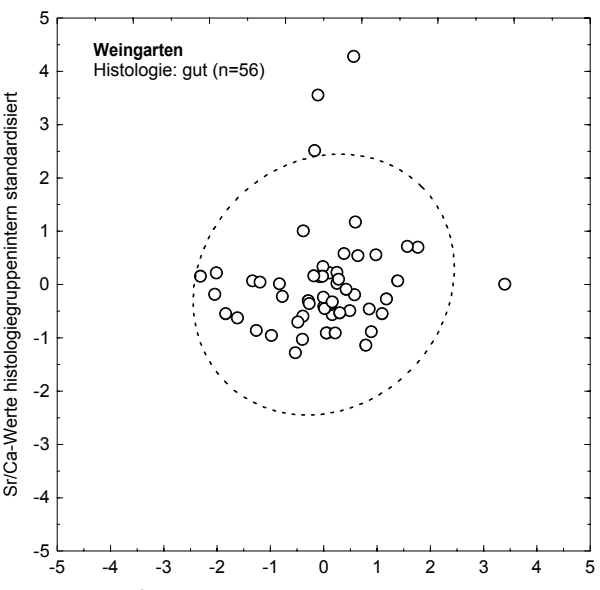

c)

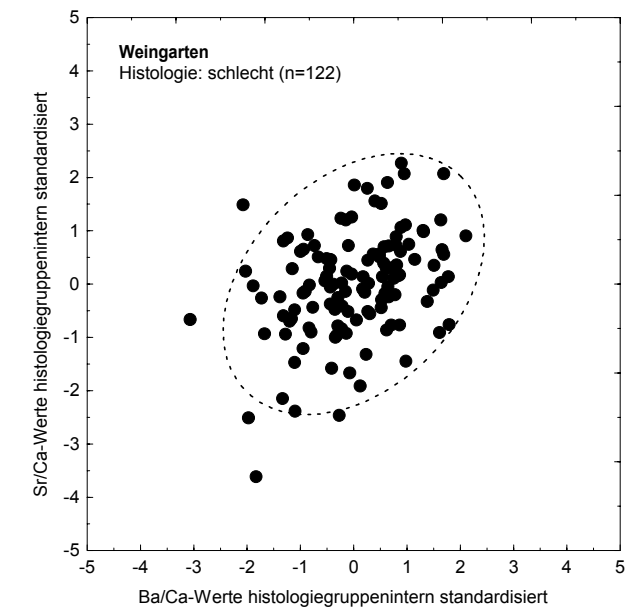

Abbildung 11: Vergleich der gruppeninternen Zusammenhänge von Ba/Ca- und Sr/Ca-Werten zwischen den Histologiegruppen für Kirchheim unter Teck (a+d), Wenigumstadt $(b+e)$ und Weingarten $(c+f)$.

Es sind jeweils die gruppenintern standardisierten Werte der populationsinternen Gruppe mit guter (a-c) und mit schlechter Histologie (d-f) dargestellt. Die eingezeichneten Ellipsen entsprechen den Konfidenzellipsen mit einem Koeffizienten von 0,95. Durch diese werden die bivariaten Verteilungscharakteristika sowie von dieser abweichende Einzelwerte veranschaulicht. (vgl. Text, S. 88 u. 90). 


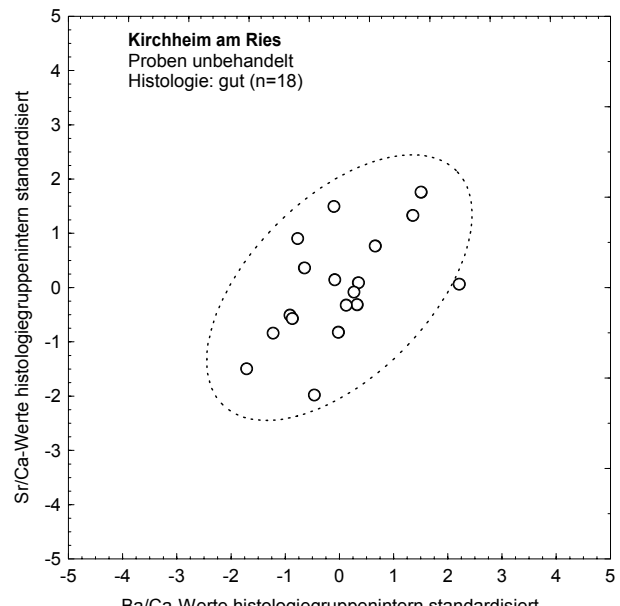

a) b)

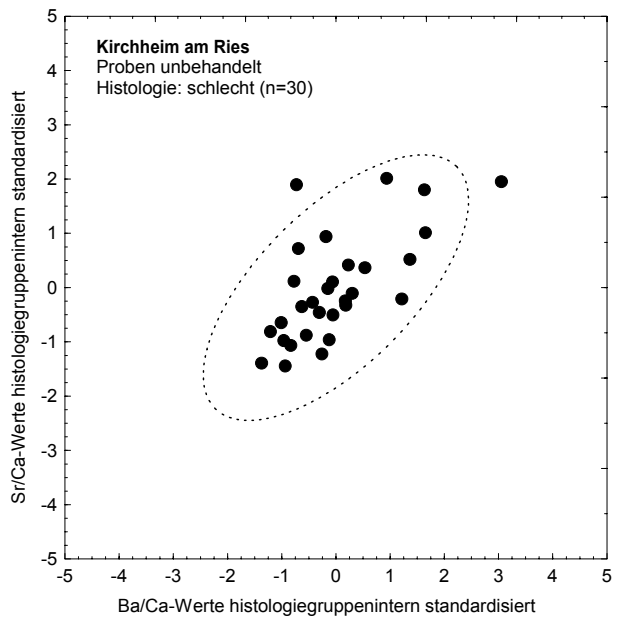

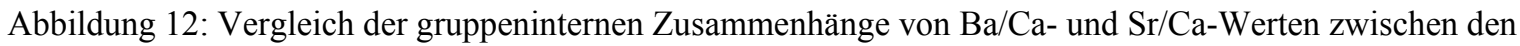
Histologiegruppen für Kirchheim am Ries.

a) Gruppe der histologisch gut erhaltenen Proben; b) Gruppe der schlecht erhaltenen Proben (zu den Darstellungsbesonderheiten vgl. Abbildung 11). Zu beachten ist, daß im Gegensatz zu den drei anderen Skelettpopulationen für Kirchheim am Ries die Werte nicht aufgereinigter Proben dargestellt sind (vgl. Text).

Für Weingarten ist ein solcher Vergleich fraglich, da bei der Gruppe der guten Histologien mehrere "auffällig verteilungsferne" Datenwerte die berechnete Konfidenzellipse verzerren. Im Hinblick auf den durch diese Werte möglichen Ausreißereinfluß auf die Korrelationen (vgl. 3.2.3.4, S. 71) wurde für diese Gruppe von Weingarten der Korrelationskoeffizient zwar berechnet, jedoch nur unter Vorbehalt dokumentiert. Es wird darauf hingewiesen, daß durch den Ausschluß der betreffenden Werte $(n=4)$ sich der Korrelationskoeffizient für die Gruppe nur unwesentlich erhöht und die Korrelation dann ebenfalls nicht signifikant ist [Korrelation innerhalb reduzierter Gruppe mit guter Histologie $(\mathrm{n}=52)$ von Weingarten: $\mathrm{r}=0,17 ; \mathrm{p}=0,10]$.

Für die drei anderen Populationen wurden die Datenverteilungen für Korrelationsanalysen als unkritisch bewertet. Die ermittelten Korrelationskoeffizienten (Produkt-Moment-Korrelation, vgl. 3.2.3.4, S. 71) sind zusammen mit den Testergebnissen hinsichtlich ihrer statistischen Signifikanz in Tabelle 4 aufgelistet. 
Tabelle 4: Korrelationsanalysen (Produkt-Moment-Korrelation) für den Zusammenhang der $\mathrm{Ba} / \mathrm{Ca}$ - und der $\mathrm{Sr} / \mathrm{Ca}-\mathrm{Werte}$.

Die für die Histologiegruppen resultierenden Korrelationskoeffizienten werden verglichen für die Populationen Kirchheim unter Teck, Wenigumstadt, Weingarten und Kirchheim am Ries

\begin{tabular}{|l|c|c|c|c|c|c|c|c|l|l|l|}
\hline \multirow{2}{*}{ Population } & \multicolumn{4}{|c|}{$\begin{array}{c}\text { Korrelation } \\
\text { innerhalb Gruppe } \\
\text { mit guter Histologie }\end{array}$} & \multicolumn{3}{|c|}{$\begin{array}{c}\text { Korrelation } \\
\text { innerhalb Gruppe } \\
\text { mit schlechter Histologie }\end{array}$} & \multicolumn{2}{|l|}{ Korrelationsvergleich } \\
\cline { 2 - 9 } & $\mathbf{n}$ & $\mathbf{r}_{\mathrm{Hg}}$ & $\mathbf{t}$ & $\mathbf{p}$ & $\mathbf{n}$ & $\mathbf{r}_{\mathrm{Hs}}$ & $\mathbf{t}$ & $\mathbf{p}$ & $\mathbf{r}_{\mathrm{Hg}}$ vs. $\mathrm{r}_{\mathrm{Hs}}$ & $\mathbf{z}$ & $\mathbf{p}$ \\
\hline $\begin{array}{l}\text { Kirchheim } \\
\text { unter Teck } \\
\text { Wenigumstadt }\end{array}$ & 28 & 0,39 & 2,14 & $0,020^{+}$ & 73 & 0,28 & 2,48 & $0,008^{+}$ & $\mathrm{r}_{\mathrm{Hg}}>\mathrm{r}_{\mathrm{Hs}}$ & 0,50 & 0,308 \\
$\begin{array}{l}\text { Weingarten } \\
\text { Kirchheim } \\
\text { am Ries }\end{array}$ & 56 & 0,49 & 2,90 & $0,004^{+}$ & 57 & 0,66 & 6,56 & $<0,001^{+}$ & $\mathrm{r}_{\mathrm{Hg}}<\mathrm{r}_{\mathrm{Hs}}$ & k.T. & k.T. \\
\hline
\end{tabular}

Werte in Klammern bei Weingarten infolge extremer Werteverteilung fraglich (vgl. Abbildung 11)

$\mathrm{r}_{\mathrm{Hg}} \quad=$ Korrelationskoeffizient Gruppe gute Histologie

$\mathrm{r}_{\mathrm{Hs}} \quad=$ Korrelationskoeffizient Gruppe schlechte Histologie

$\mathrm{n} \quad=$ Stichprobengröße

k.T. $\quad=$ kein Test durchgeführt

${ }^{+}$bei festgelegtem $\alpha=0,05$ signifikant

Der theoretischen Vorannahme folgend, ist ein Vergleich der Korrelationen zwischen den Histologiegruppen nur sinnvoll, wenn der Koeffizient der Gruppe der histologisch gut erhaltenen Proben größer als jener der schlecht erhaltenen ist. Wie Tabelle $4 \mathrm{zu}$ entnehmen, trifft dies nur für Kirchheim unter Teck zu. Der Test ergab aber auch für diese Population keinen statistisch abgesicherten Unterschied zwischen den Korrelationen der beiden Histologiegruppen $(\mathrm{p}=0,31)$.

Die Untersuchung des Barium-Strontium-Zusammenhanges hat damit für alle vier untersuchten Populationen keine der theoretischen Vorannahme entsprechende Differenzierung der histologischen Gruppen ergeben. Histologisch schlecht erhaltene Knochenproben, die nach den Ergebnissen von 4.1 (vgl. zusammenfassend S. 80f) sich von gut erhaltenen systematisch durch höhere $\mathrm{Ba} / \mathrm{Ca}$ - und $\mathrm{Sr} / \mathrm{Ca}$-Wert unterscheiden und daher als chemisch stärker verändert einzuschätzen sind, zeigen keine veränderte (schwächere) Korrelationscharakteristik der beiden Konzentrationsverhältnisse. Hervorzuheben ist, daß dies auch für die beiden Histologiegruppen der Proben ohne jegliche Probenaufreinigung von Kirchheim am Ries zutrifft (vgl. zu diesem Ergebnis 4.4.2). 


\section{Ca/P-Verhältnis}

Entsprechend den Herleitungen (vgl. 2.3, S. 28f) zur Verwendung des Ca/P-Verhältnisse als ein empfohlenes mineralchemisches Kontrollkriterium wurden in allen vier untersuchten Populationen die beiden Histologiegruppen diesbezüglich jeweils miteinander verglichen. Ausgangspunkt hierfür sind die systematischen, histologieabhängigen Unterschiede der Spurenelementkonzentrationen innerhalb der Populationen (höhere $\mathrm{Ba} / \mathrm{Ca}$ - und $\mathrm{Sr} / \mathrm{Ca}$ Werte in der Gruppe der schlecht erhaltenen Proben, vgl. 4.1, S. 80f). Hieraus abgeleitet wird die Hypothese einer systematischen chemischen Veränderung des Minerals, welche eine gerichtete Beeinflussung des $\mathrm{Ca} / \mathrm{P}-$ Verhältnisses wahrscheinlich macht. Letztere sollte über einen Unterschied zwischen den $\mathrm{Ca} / \mathrm{P}-$ Werten der beiden Histologiegruppen meßbar sein.

In Abbildung 13 sind für die $\mathrm{Ca} / \mathrm{P}-$ Werte die deskriptiv statistischen Kennwerte der histologischen Gruppen der vier untersuchten Populationen dargestellt ${ }^{4}$. Hier ist zu beachten, daß für Kirchheim am Ries (Abbildung 13d) wieder die Daten der Proben ohne jegliche Aufreinigung dargestellt sind. Dies erfolgte unter der Annahme, daß diese ohne chemische Veränderung der Aufreinigung die diagenetischen Mineralveränderungen am deutlichsten abbilden sollten. In dieser Teilabbildung zu Kirchheim am Ries sind zum Vergleich zusätzlich Werte von rezenten Knochenproben eingezeichnet (eigene Analysen eines rezenten Femurs, vgl. 4.5 und Daten nach Gawlik et al. 1982 berechnet). Die gleiche Skalierung in allen Teilabbildungen ermöglicht den Vergleich zwischen den Populationen.

Ein erster Vergleich läßt keine auffälligen Diskrepanzen hinsichtlich der zentralen Tendenzen der Werte erkennen, weder innerhalb der Populationen noch zwischen diesen. Festzustellen ist nur eine höhere Variabilität (Standardabweichung und Spannweite) bei den histologisch schlecht erhaltenen Knochenproben. Die Mittelwerte und Standardabweichungen aller Gruppen der Populationen können jedoch in den Bereich der Spannweite von

\footnotetext{
${ }^{4}$ Anmerkung zu Stichprobenunterschieden bei den folgenden Auswertungen:

Für die Auswertungen weiterer Kontrollkriterien (Ca/P-Verhältnis, Magnesium-, Aluminiumkonzentration und Masseverluste während der Probenvorbereitung) standen nicht immer für alle untersuchten Populationen vollständige Datensätze zur Verfügung. Ursachen hierfür sind die in den früheren Untersuchungen diesbezüglich nur teilweise erhobenen Daten. Hieraus resultieren variierende Stichprobenzahlen für die einzelnen Variablen bzw. es mußte für einige Variablen auf die Auswertung von Populationen infolge zu geringer Stichprobengröße ganz verzichtet werden. Andererseits sind die Magnesium- und Aluminiumkonzentrationen nur für Kirchheim am Ries bestimmt worden.

In allen Abbildungen und Tabellen sind die "variierenden" Stichprobenzahlen angegeben. Es handelt sich hierbei immer um zufällige Ausschnitte der bisher ausgewerteten Datengruppen.
} 
rezenten Proben (2,0 bis 2,36, nach Gawlik et al. 1982) eingeordnet werden bzw. gehen nur unwesentlich darüber hinaus.

a)
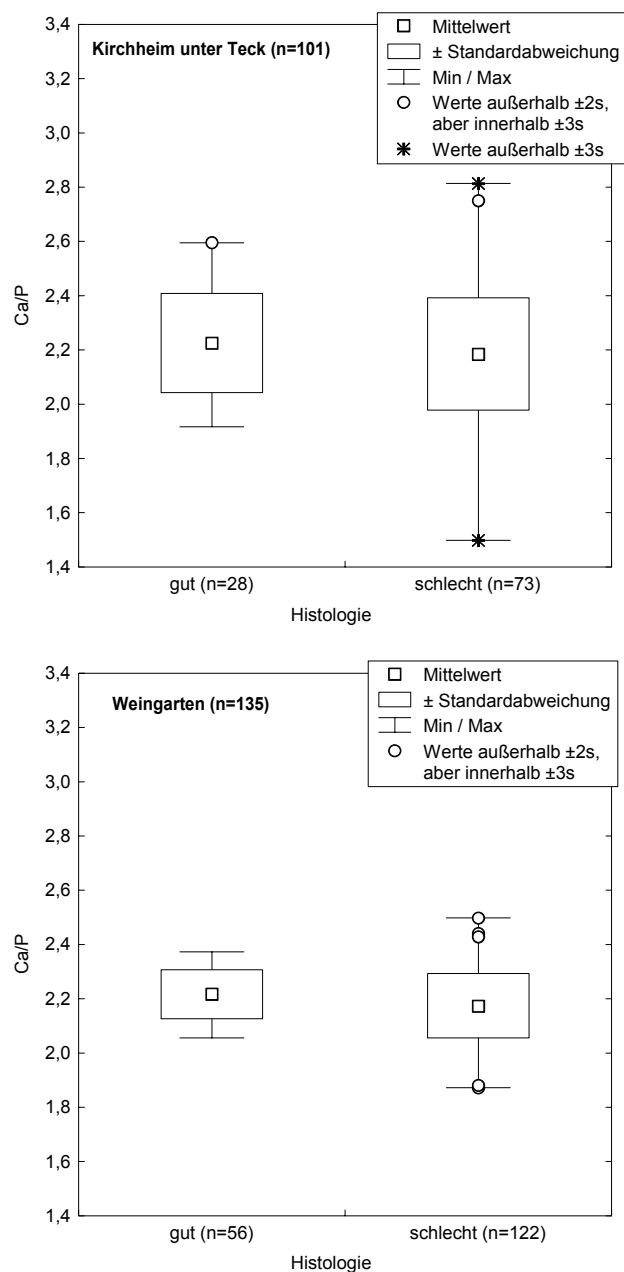

b)
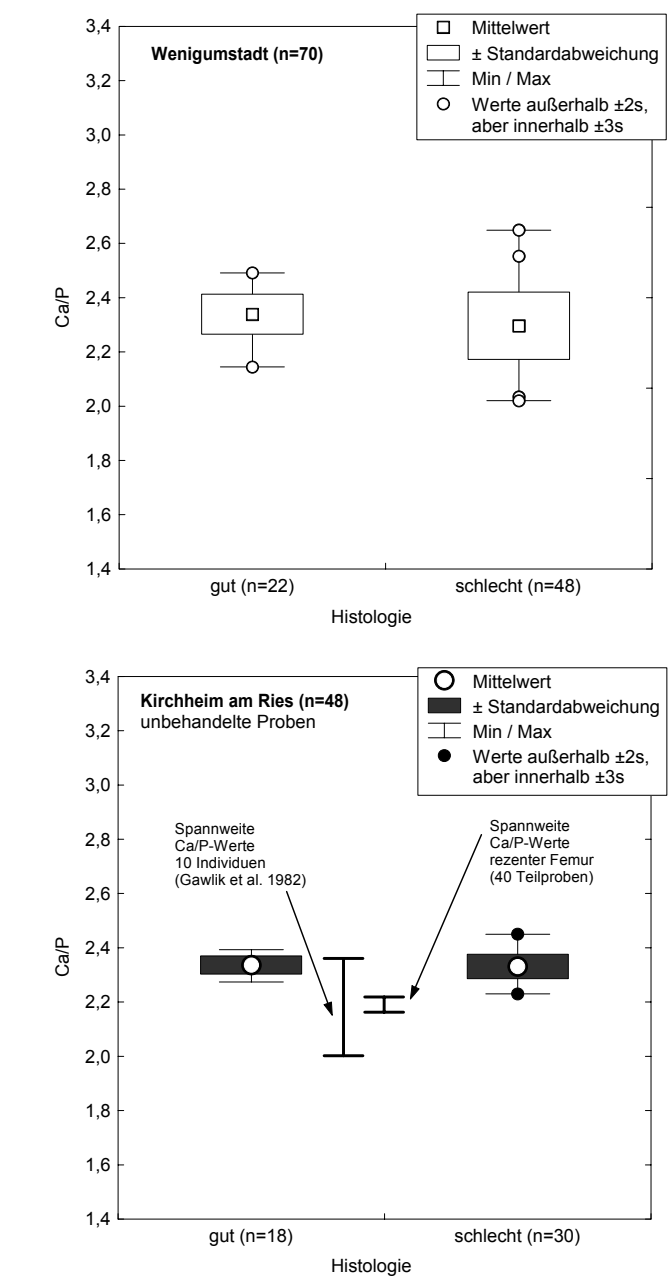

d)

Abbildung 13: Statistisch deskriptiver Vergleich der Ca/P-Werte zwischen den Histologiegruppen für a) Kirchheim unter Teck, b) Wenigumstadt, c) Weingarten und d) Kirchheim am Ries.

In den Teilabbildungen sind jeweils die populationsinternen Gruppenvergleiche dargestellt. Die gleiche Skalierung ermöglicht aber auch den Vergleich zwischen den drei Populationen. Zu beachten ist, daß für Kirchheim am Ries im Gegensatz zu den drei anderen Skelettpopulationen die Ca/P-Werte nicht aufgereinigter Proben dargestellt sind. Die in d) eingezeichnete Spannweite der $\mathrm{Ca} / \mathrm{P}-$ Werte eines rezenten Femurs resultiert aus den Werten der 40 Teilproben der Untersuchung zur Erfassung der intraossären Konzentrationsvariabilität. Die Angabe der Spannweite für die Daten von Gawlik et al. (1982) resultiert aus der Umrechnung der Rohdaten (vgl. Kapitel 4.5).

Die Testergebnisse der statistischen Vergleiche (Mann-Whitney-U-Test, vgl. 3.2.3.4, S. 70) zwischen den Histologiegruppen entsprechen der graphischen Einschätzung (vgl. Tabelle 5). Nur für Weingarten kann ein Unterschied zwischen den beiden Gruppen angenommen werden $(p=0,04)$. Bei den drei anderen Populationen ist davon auszugehen, daß Kno- 
chenproben mit differentiellem histologischen Erscheinungsbild keine systematisch unterschiedlichen $\mathrm{Ca} / \mathrm{P}-$ Werte haben.

Es wird nochmals darauf hingewiesen, daß die für Kirchheim am Ries verglichenen Proben unbehandelt waren. Diagenetisch unterschiedlich veränderte bodengelagerte Knochenproben unterscheiden sich hiernach primär nicht hinsichtlich ihres $\mathrm{Ca} / \mathrm{P}-\mathrm{Quotienten} \mathrm{(vgl.} \mathrm{zu}$ diesem Ergebnis 4.4.1)!

Tabelle 5: Vergleiche der Histologiegruppen hinsichtlich der Ca/P-Werte (Mann-Whitney-U-Test)

\begin{tabular}{|l|r|r|l|l|}
\hline \multirow{2}{*}{ Population } & \multicolumn{2}{|c|}{ Gruppengrößen } & \multicolumn{2}{c|}{ Ca/P-Gruppenvergleich } \\
\cline { 2 - 5 } & $\begin{array}{c}\text { gute } \\
\text { Histologie }\end{array}$ & $\begin{array}{c}\text { schlechte } \\
\text { Histologie }\end{array}$ & Z-Wert & p-Wert \\
\hline Kirchheim unter Teck & 28 & 73 & 0,94 & 0,347 \\
Wenigumstadt & 29 & 57 & 1,87 & 0,061 \\
Weingarten & 35 & 100 & 2,02 & $0,043^{+}$ \\
Kirchheim am Ries & 18 & 30 & 0,64 & 0,523 \\
\hline
\end{tabular}

${ }^{+}$bei festgelegtem $\boldsymbol{\alpha}=\mathbf{0 , 0 5}$ signifikant

\section{Mg-Konzentration}

Konzentrationsunterschiede zwischen gut erhaltenen und schlecht erhaltenen Knochenproben sind infolge der erhöhten Löslichkeit der magnesiumhaltigen Mineralphase wahrscheinlich (vgl. 2.3, S.30).

Magnesiumdaten standen für die Populationen der früheren Untersuchungen (Kirchheim unter Teck, Wenigumstadt und Weingarten) nicht zu Verfügung. Für Kirchheim am Ries wurden die Magnesiumgehalten für unbehandelte Knochenproben und zusätzlich für Probenpendants nach verschiedenen Aufreinigungsmethoden bestimmt. Dies entspricht der Vorgehensweise der Untersuchungen zur Probenaufreinigung (vgl. 4.2). Die statistische Auswertung erfolgte daher der dortigen analog auf der Grundlage eines Meßwiederholungsdesigns. Es werden die beiden Histologiegruppen hinsichtlich der $\mathrm{Mg} / \mathrm{Ca}-\mathrm{Werte}$ verglichen und gleichzeitig werden die Konzentrationsdaten der Knochenproben vor und nach den verschiedenen Aufreinigungsmethoden gegenübergestellt. Durch den zweiten Vergleich wird der Einfluß der Probenaufreinigung auf die $\mathrm{Mg} / \mathrm{Ca}-$ Werte erfaßt. Zusätzlich wird die Interaktion zwischen Histologie und Aufreinigung statistisch geprüft. Eine hierdurch "meßbare" histologieabhängige, differentielle Aufreinigungswirkung wird als Indiz für spezifische Veränderungen der Knochensubstanz ausgewertet. 


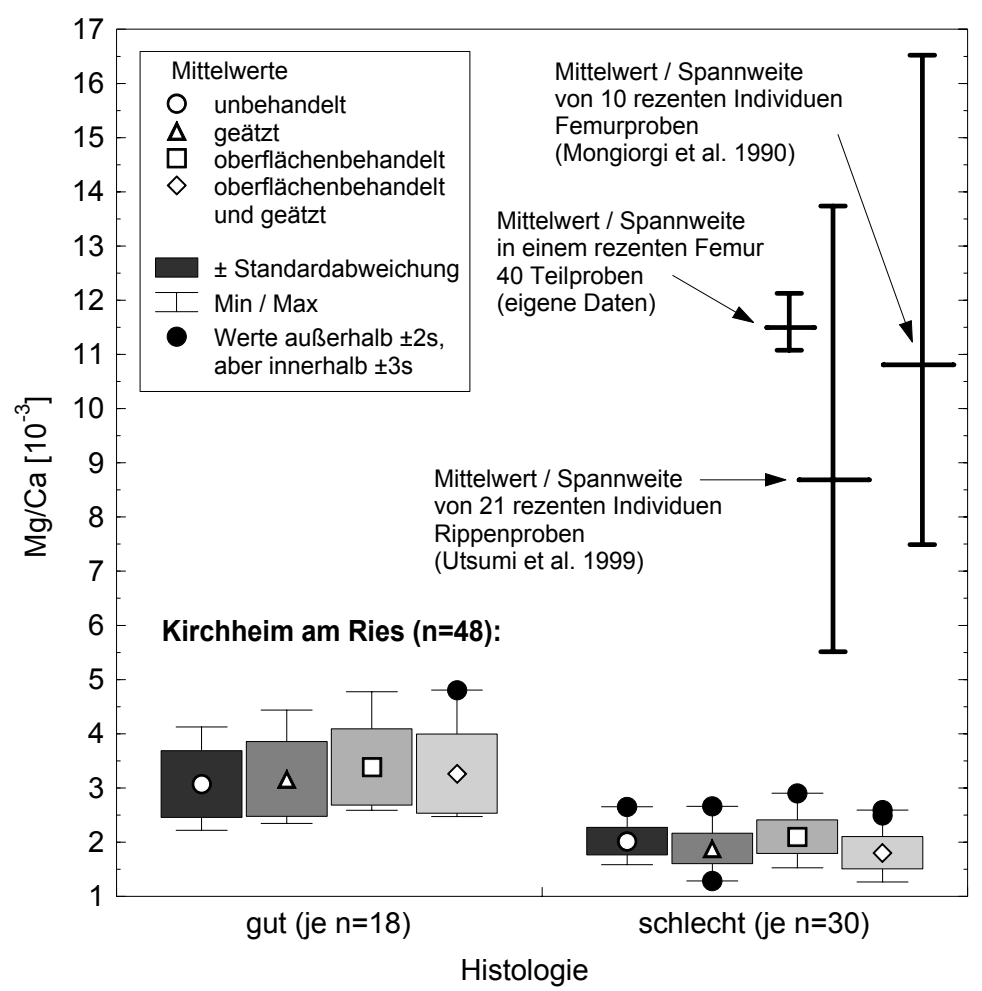

Abbildung 14: Statistisch deskriptiver Vergleich der $\mathrm{Mg} / \mathrm{Ca}$-Werte zwischen den Histologiegruppen von Kirchheim am Ries.

Durch die in der Legende den Mittelwerten zugeordneten Symbole und die dazugehörigen Schattierungen der Boxen werden die beiden diagenetischen Gruppen für die verschiedenen Aufreinigungsvarianten jeweils getrennt dargestellt. Zum Vergleich sind Mittelwerte und Spannweite der $\mathrm{Mg} / \mathrm{Ca}-$ Werte des zur Erfassung der intraossären Konzentrationsvariabilitäten untersuchten rezenten Femurs (vgl. Abbildung 31) sowie $\mathrm{Mg} / \mathrm{Ca}$-Daten aus der Literatur dargestellt (Utsumi et al. 1999; Mongiorgi et al. 1990, aus Absolutkonzentrationen errechnet).

In Abbildung 14 ist zunächst der deskriptive Vergleich der $\mathrm{Mg} / \mathrm{Ca}-$ Werte dargestellt. Es sind die Daten beider Histologiegruppen einmal für den unbehandelten Probensatz sowie für jede Aufreinigungsmethoden dargestellt. Zusätzlich enthält diese Grafik Vergleichsdaten eigener Analysen eines rezenten Femurs (vgl. 4.5) sowie Literaturdaten (Utsumi et al. 1999; Mongiorgi et al. 1990, für diese wurden die Konzentrationsquotienten aus den dokumentierten Rohdaten berechnet).

Der graphische Vergleich zeigt für rezente Daten zwar eine große Variabilität, doch liegen die niedrigsten Rezentwerte immer noch über den höchsten Werten von Kirchheim am Ries. Der populationsinterne Vergleich der Histologiegruppen von Kirchheim am Ries läßt für gut erhaltene Knochenproben gegenüber schlecht erhaltenen höhere Konzentrationen bei gleichzeitig größerer Variabilität (Standardabweichung) erkennen. Die Aufreinigungsmethoden führen zwar zu veränderten Konzentrationswerten, aber die beschriebene Vergleichscharakteristik der Histologiegruppen ist für unbehandelte und verschieden aufgereinigte Probensätze gleich.

Diese Deskription findet in der statistischen Überprüfung ihre Entsprechung. Den Ergebnissen in Tabelle 6 zufolge ist erstens ein globaler Unterschied zwischen den Histologiegruppen hinsichtlich der $\mathrm{Mg} / \mathrm{Ca}$-Werte wahrscheinlich, zweitens wirken die Aufreinigungsmethoden konzentrationsverändernd und drittens sind diese Konzentrationsänderungen für die Histologiegruppen nicht gleich (für alle drei Aussagen $p<0,001$ ). 
Tabelle 6: Statistische Überprüfungen für die $\mathrm{Mg} / \mathrm{Ca}$-Werte von Kirchheim am Ries.

Durch nichtparametrische Varianzanalyse mit Meßwiederholung werden die Histologiegruppen verglichen, der Einfluß der Aufreinigungsmethoden überprüft und die histologiespezifische Aufreinigungswirkung getestet (Interaktion Histologie-Aufreinigung).

\begin{tabular}{|l|c|l|}
\hline \multirow{2}{*}{ Faktor / Interaktion } & \multicolumn{2}{c|}{ Mg/Ca } \\
\cline { 2 - 3 } & B-Wert & \multicolumn{1}{c|}{ p-Wert } \\
\hline Histologie [gut = 18; schlecht $=30]$ & 131,61 & $<0,001^{+}$ \\
Aufreinigung [Methoden $=3+1$ (unbehandelt)] & 47,35 & $<0,001^{+}$ \\
Histologie - Aufreinigung & 33,02 & $<0,001^{+}$ \\
\hline
\end{tabular}

bei festgelegtem $\boldsymbol{\alpha}=\mathbf{0 , 0 5}$ signifikant

Der statistisch abgesicherte globale Unterschied der $\mathrm{Mg} / \mathrm{Ca}$-Werte zwischen den Histologiegruppen zeigt, daß über dieses Kontrollkriterium diagenetisch unterschiedlich veränderter Knochenproben differenziert werden.

Der histologiespezifische Aufreinigungseffekt (Interaktion zwischen Histologie und Aufreinigung) erlangt im Kontext einer erweiterten Charakterisierung diagenetischer Prozesse Bedeutung. In Abbildung 15 erfolgt daher eine detaillierte Darstellung dieses Phänomens.

Die Darstellungen in Abbildung 15b) und c) zeigen für beide Aufreinigungsmethoden mit chemischer Komponente (Ätzen) einen charakteristischen Unterschied zwischen den Wirkungen auf Proben der beiden Histologiegruppen. Ätzen hat bei Proben mit schlechter histologischer Erhaltung typischerweise eine Verringerung der $\mathrm{Mg} / \mathrm{Ca}-W e r t e$ zur Folge, wohingegen die Werte der gut erhalten Proben meist größer werden. Eine alleinige Oberflächenbehandlung (Entfernung der oberflächennahen Schichten) hat nach Teilgrafik b) auf die $\mathrm{Mg} / \mathrm{Ca}$-Werte beider Histologiegruppen eine gleichgerichtete und damit indifferent erhöhende Wirkung.

Als entscheidende Ergebnisse für Magnesium sind die deutliche Differenzierung der beiden Histologiegruppen hinsichtlich der $\mathrm{Mg} / \mathrm{Ca}-$ Werte (gut = höhere bzw. schlecht = niedrigere), die allgemein niedrigeren $\mathrm{Mg} / \mathrm{Ca}$ Werte der bodengelagerten Knochenproben im Vergleich zu rezentem Knochenmaterial sowie der verstärkte Magnesiumverlust der histologisch schlecht erhaltenen Knochenproben durch die Säurebehandlung zusammenzufassen (vgl. zu diesen Ergebnissen 4.4.1). 

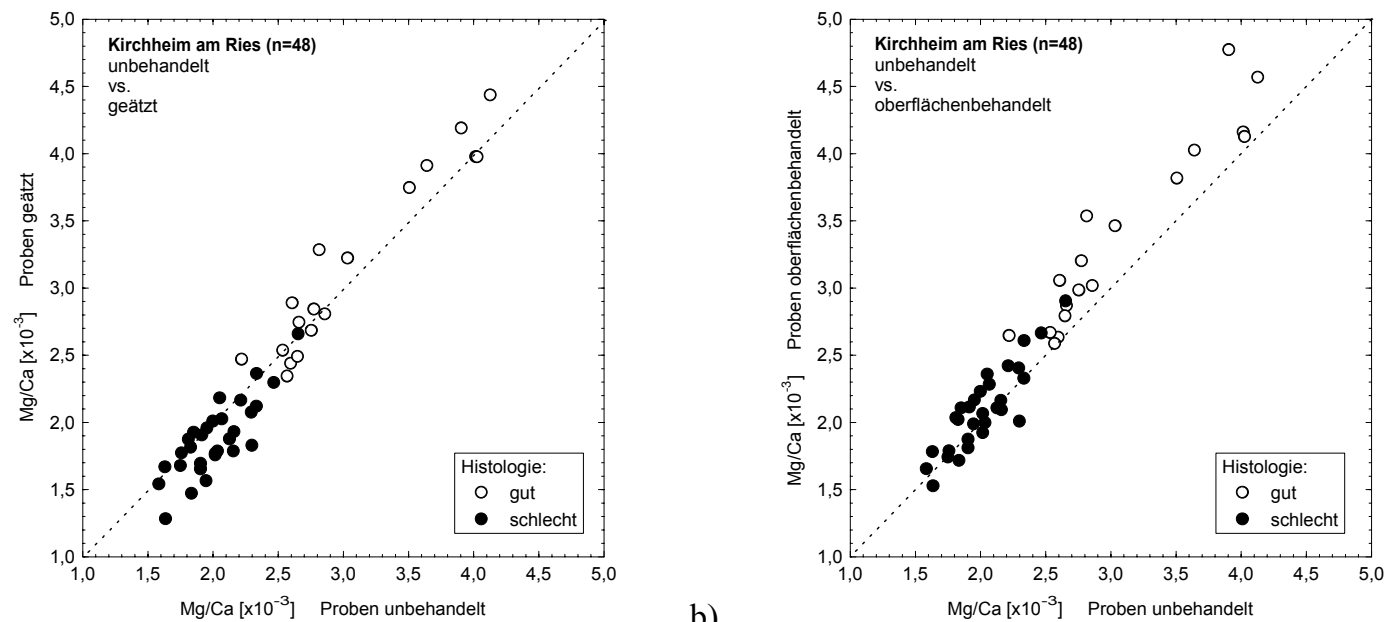

a)

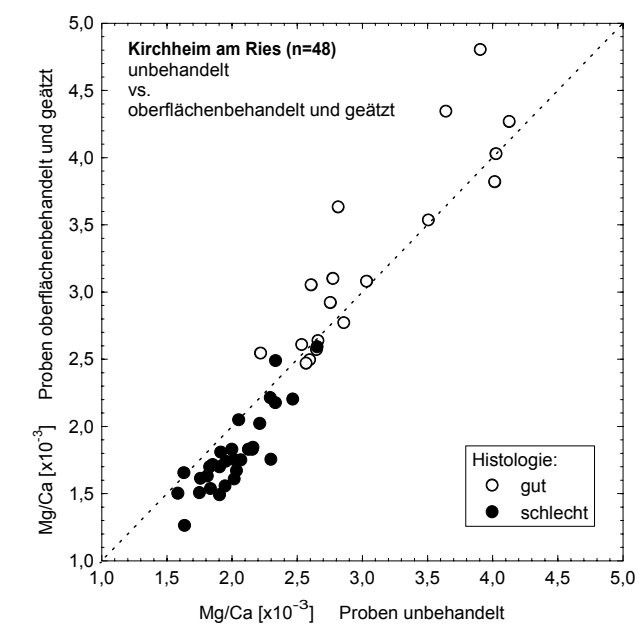

b)

c)

Abbildung 15: Aufreinigungsbedingte Veränderungen der Mg/Ca-Werte für die Knochenproben von Kirchheim am Ries.

In a), b) und c) sind jeweils die Daten der unbehandelten Proben gegen die Daten einer der Aufreinigungsmethoden aufgetragen. Damit erfolgt die Darstellung der Konzentrationsänderungen der Einzelproben über die vertikalen Abweichungen ihrer Datenpunkte von der Grafikdiagonalen. Die gestrichelte Diagonale markiert die "Neutrale". Alle Proben mit Werten auf dieser Geraden sind durch die jeweilige Probenvorbehandlung nicht beeinflußt worden.

\section{Al-Konzentration}

Aluminium ist in vivo im Knochen nur in sehr geringen Konzentrationen vorhanden bzw. bei den im Rahmen dieser Untersuchung gewählten Analysebedingungen unterhalb der Nachweisgrenze (vgl. 4.5). Nachweisbare oder deutlich erhöhte Konzentrationen sind als knochenfremd zu betrachten. Aluminium konnte daher als diageneseanzeigendes Element ausgewertet werden (vgl. 2.3, S. 31).

Aluminiumkonzentrationen wurden nur für die Skelettpopulation von Kirchheim am Ries bestimmt. Dies jedoch wie bereits bei Magnesium im Kontext der vergleichenden Untersuchungen zur Probenaufreinigung. Es konnten daher die Al/Ca-Werte vor und nach den Probenaufreinigungen verglichen werden. Die Auswertung erfolgt ebenfalls über eine Va- 
rianzanalyse zur Erfassung der Histologiegruppenunterschiede einerseits und der Aufreinigungsauswirkungen andererseits.

Die statistisch deskriptive Darstellung in Abbildung 16 zeigt für schlecht erhaltene Proben gegenüber den gut erhaltenen höhere $\mathrm{Al} / \mathrm{Ca}-W e r t e$. Allerdings ist eine nahezu vollständige Überlappung der Wertebereiche beider Gruppen festzustellen. Dies trifft insbesondere für die Gruppenvergleiche der Probensätze der verschiedenen Aufreinigungsmethoden zu. Hervorzuheben ist, daß jede Aufreinigungsmethode für einige sowohl gut als auch schlecht erhaltene Knochenproben Aluminiumkonzentrationen unterhalb der Nachweisgrenze respektive Al/Ca-Werte von Null zur Folge hatte. Allgemein werden die Al/Ca-Werte beider Histologiegruppen durch jede Aufreinigungsmethode verringert.

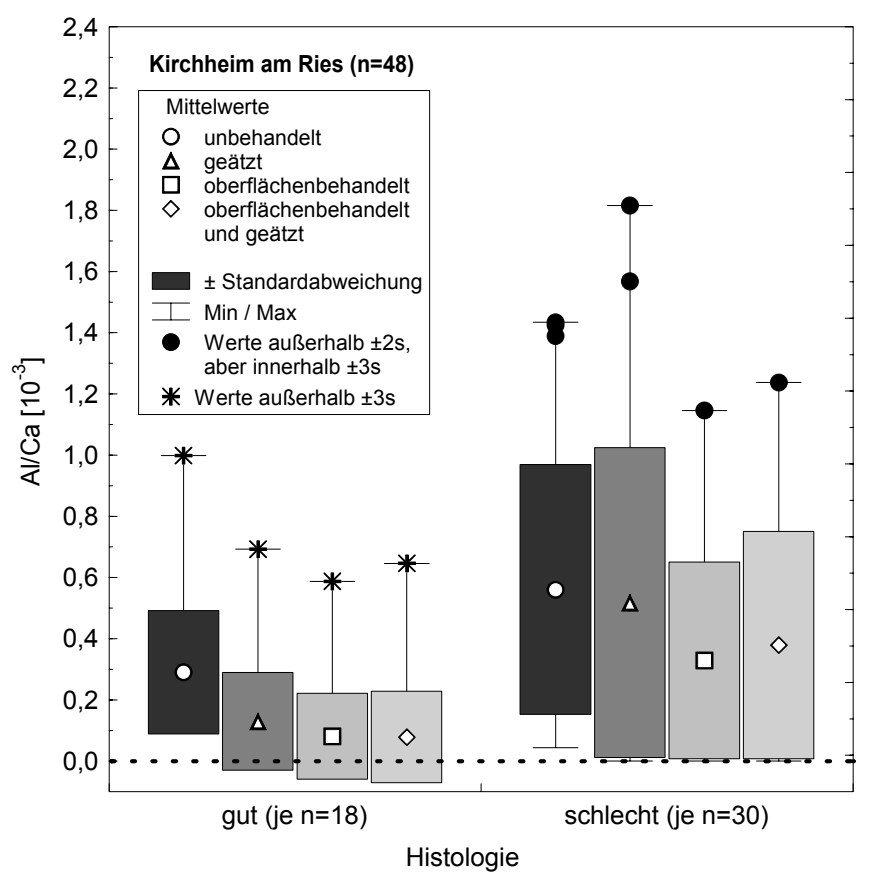

Abbildung 16: Vergleich der Al/Ca-Werte zwischen den Histologiegruppen von Kirchheim am Ries vor und nach verschiedenen Methoden der Probenaufreinigung.

a) Statistisch deskriptiver Vergleich: Durch die in der Legende den Mittelwerten zugeordneten Symbole und die dazugehörigen Schattierungen der Boxen werden die beiden diagenetischen Gruppen für die verschiedenen Aufreinigungsvarianten jeweils getrennt dargestellt. Nach den Aufreinigungen enthielten einige Proben nur noch Aluminium im Bereich der Nachweisgrenze oder darunter. Die resultierenden Konzentrationsquotienten sind daher Nullwerte (respektive Minima). Gleichzeitig sind durch die einzelnen (bzw. maximal zwei innerhalb einer Gruppe!) Ausreißer und Extreme die Werteverteilungen verzerrt. Daher reichen die Standardabweichungen in den negativen Wertebereich hinein.

Aus der statistischen Prüfung resultieren für die Al/Ca-Werte die in Tabelle 7 aufgelisteten Ergebnisse. Hiernach gibt es für die Histologiegruppen einen globalen Unterschied $(\mathrm{p}<0,001)$. Wie nach der Deskription zu erwarten, haben auch die Aufreinigungsmethoden signifikante Konzentrationsänderungen zur Folge $(\mathrm{p}<0,001)$. 
Tabelle 7: Statistische Überprüfungen für die Al/Ca-Werte von Kirchheim am Ries.

Durch nichtparametrische Varianzanalyse mit Meßwiederholung werden die Histologiegruppen verglichen, der Einfluß der Aufreinigungsmethoden überprüft und die histologiespezifische Aufreinigungswirkung getestet (Interaktion Histologie-Aufreinigung).

\begin{tabular}{|l|c|l|}
\hline \multirow{2}{*}{ Faktor $/$ Interaktion } & \multicolumn{2}{c|}{ Al/Ca } \\
\cline { 2 - 3 } & B-Wert & \multicolumn{1}{c|}{ p-Wert } \\
\hline Histologie $\quad[$ gut $=18 ;$ schlecht $=30]$ & 17,02 & $<0,001^{+}$ \\
Aufreinigung [Methoden $=3+1$ (unbehandelt)] & 93,66 & $<0,001^{+}$ \\
Histologie - Aufreinigung & 10,95 & $<0,001^{+}$ \\
\hline
\end{tabular}

bei festgelegtem $\boldsymbol{\alpha}=\mathbf{0 , 0 5}$ signifikant

Das Ergebnis einer statistisch wahrscheinlichen Interaktion zwischen Histologie und Aufreinigung $(\mathrm{p}<0,001)$ wird in den Grafiken der Abbildung 17 veranschaulicht. Die differenzierte Wirkung der Aufreinigung ist hierbei dahingehend auszuwerten, daß die $\mathrm{Al} / \mathrm{Ca}-$ Werte der gut erhaltenen im Gegensatz zu den schlecht erhaltenen Proben einheitlicher verringert werden.

Beachtenswert ist Abbildung 17b. Im Vergleich zu den uneinheitlicheren Auswirkungen der anderen Aufreinigungsvarianten führt die Oberflächenbehandlung (Entfernung oberflächennaher Schichten) für fast jede Knochenprobe zur Verringerung des $\mathrm{Al} / \mathrm{Ca}-W e r t e s$ respektive der Aluminiumkonzentration.

Eine vollständige Entfernung des Aluminiums aus den Knochenproben konnte aber nicht für alle Proben erreicht werden. Entsprechend bleibt für Aluminium festzustellen, daß sich gut und schlecht erhaltene Proben hinsichtlich einer Anreicherung diese Elementes zwar unterscheiden, Aluminium aber auch in gut erhaltenen Proben vorhanden ist und die verwendeten Methoden der Aufreinigung eine vollständige Entfernung nicht gewährleisten können. 
a)
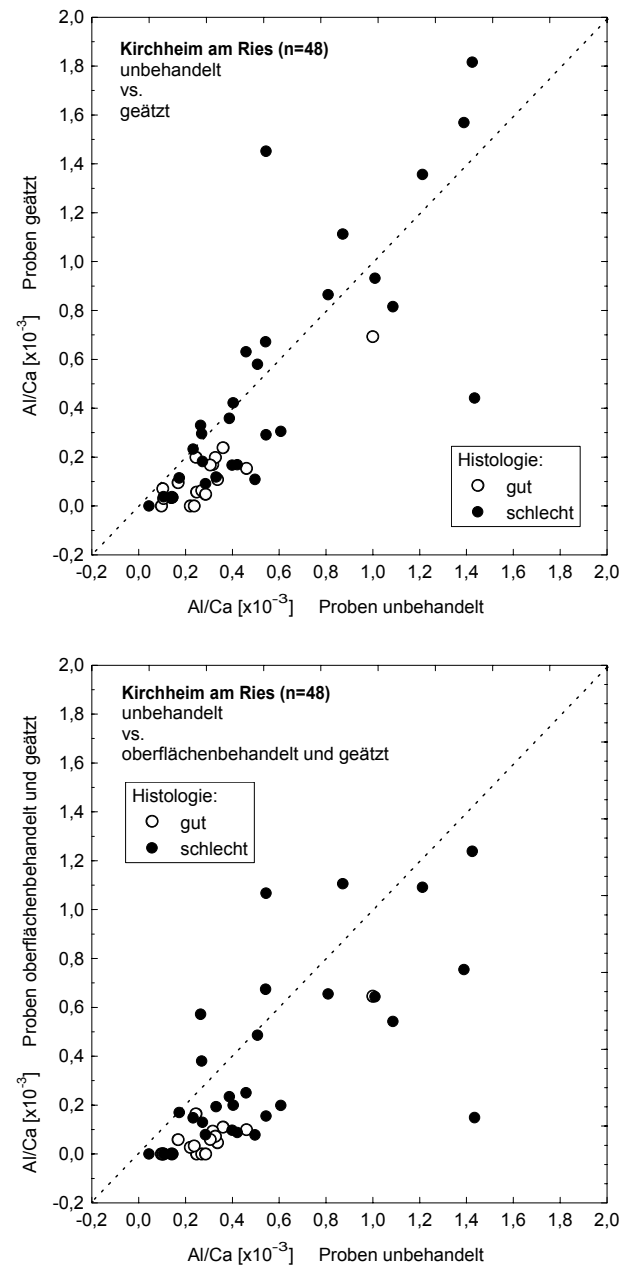

c)

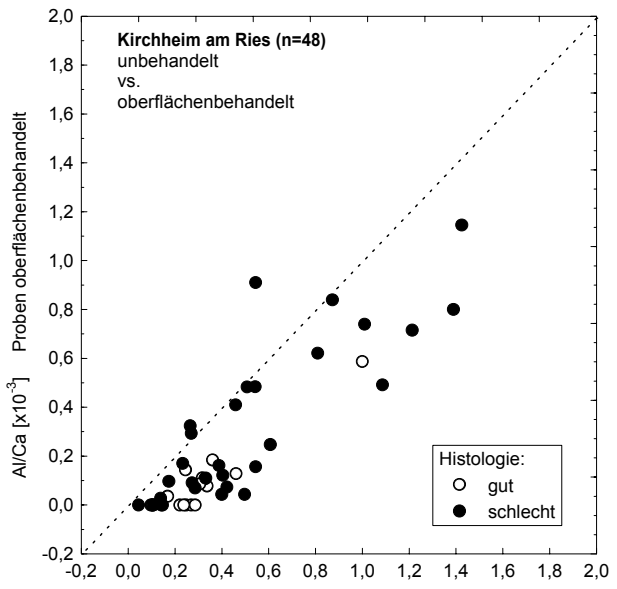

b)

Abbildung 17: Aufreinigungsbedingte Veränderungen der Al/Ca-Werte für die Knochenproben von Kirchheim am Ries.

In a), b) und c) sind jeweils die Daten der unbehandelten Proben gegen die Daten einer der Aufreinigungsmethoden aufgetragen. Damit erfolgt die Darstellung der Konzentrationsänderungen der Einzelproben über die vertikalen Abweichungen ihrer Datenpunkte von der Grafikdiagonalen. Die gestrichelte Diagonale markiert die "Neutrale". Alle Proben mit Werten auf dieser Geraden sind durch die jeweilige Probenvorbehandlung nicht beeinflußt worden.

\section{Masseverlust bei der Probenaufreinigung durch Ätzen}

In den vorangegangenen Auswertungen wurden nur elementanalytische Parameter hinsichtlich ihres Differenzierungsvermögens zwischen histologisch definierten bzw. diagenetisch bedingten Gruppen von Knochenproben untersucht. Mit den beiden folgenden spezifischen Masseverlusten (während des Ätzens bzw. während des Veraschens, vgl. S. 102ff) werden Daten aus der Probenvorbereitung ausgewertet. Für beide Masseverluste sind nach den Vorüberlegungen in 2.3 (S. 31ff) Hypothesen für spezifische Unterschiede zwischen den Histologiegruppen abgeleitet worden.

Daten für den Masseverlust beim Ätzen konnten in hinreichendem Umfang nur für Wenigumstadt, Weingarten und Kirchheim am Ries ausgewertet werden. In Abbildung 18 wer- 
den für diese drei Skelettpopulationen die Histologiegruppen verglichen. Dargestellt sind die statistisch deskriptiven Kenngrößen für die relativen Masseverluste der Knochenproben. Für alle drei untersuchten Populationen sind für die schlecht erhaltenen Knochenproben höhere Masseverluste zu beobachten.

a)

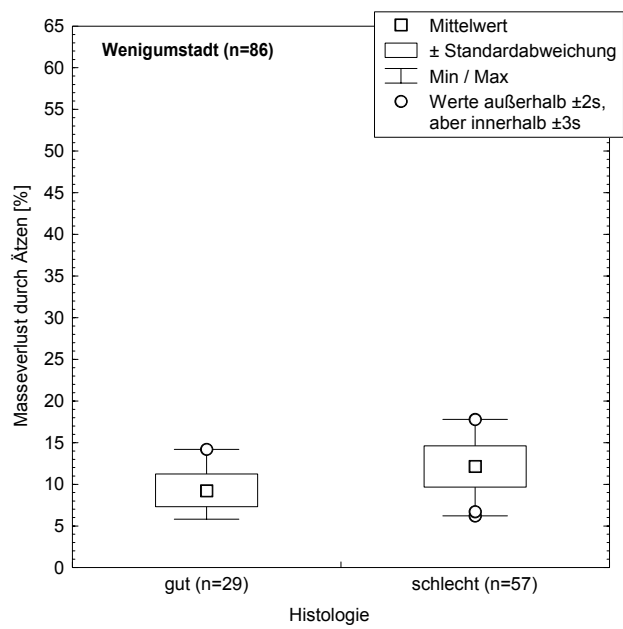

b)
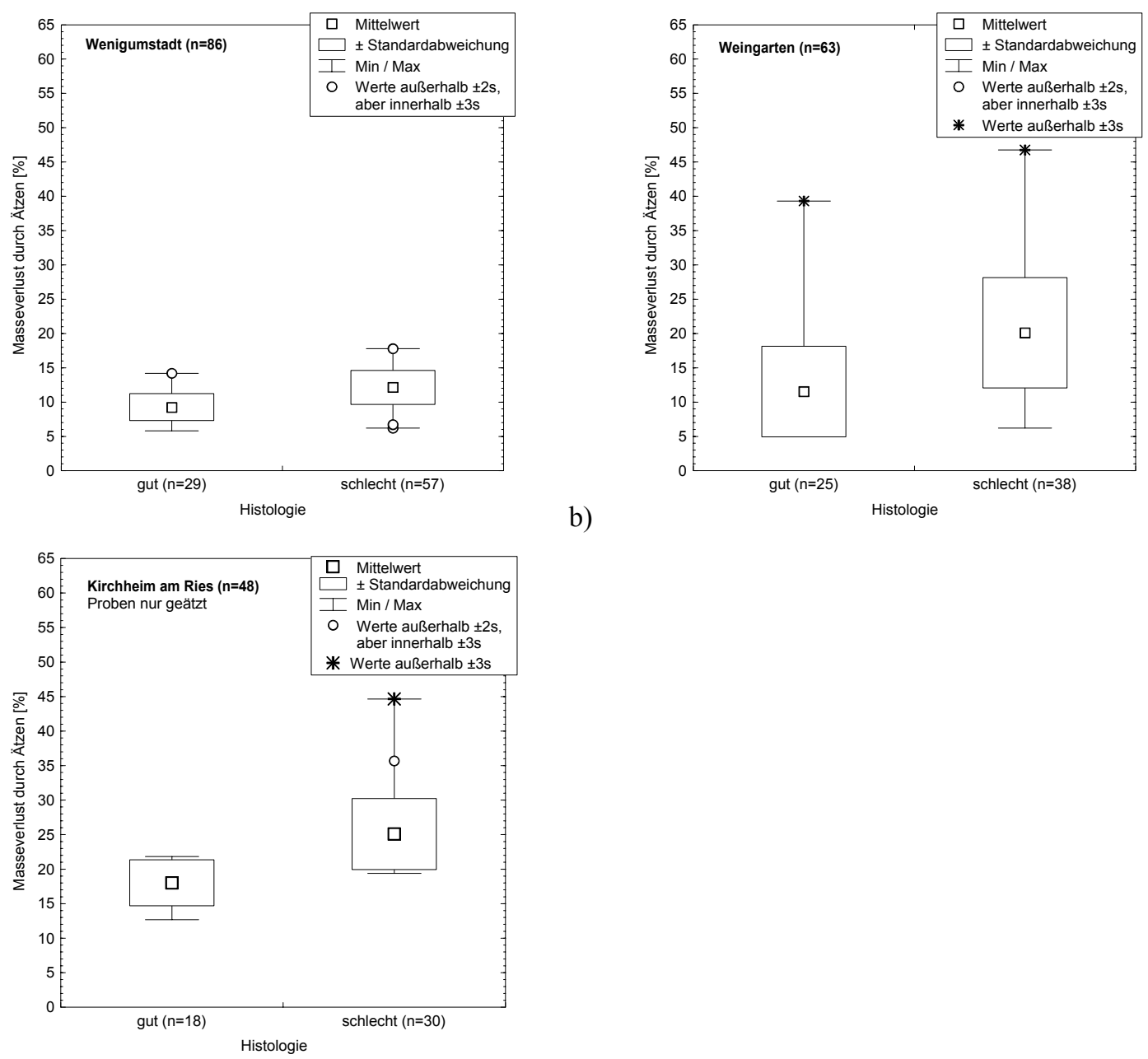

c)

Abbildung 18: Statistisch deskriptiver Vergleich der durch das Ätzen bedingten Masseverluste zwischen den Histologiegruppen

a) Wenigumstadt, b) Weingarten und c) Kirchheim am Ries. Die für Kirchheim am Ries dargestellten Masseverluste sind von dem nur geätzten Probensatz (ohne mechanische Oberflächenbehandlung).

Diese Vergleiche sind für Wenigumstadt und Weingarten nur unter Vorbehalt auszuwerten. Für diese Serien war die Ätzdauer der Knochenproben nicht definiert, sondern wurde der Kompaktastärke entsprechend variiert (vgl. 3.2.1.3, S. 46f). Die signifikanten Ergebnisse dieser beiden Populationen sind in Tabelle 8 unter Vorbehalt aufgeführt. Für Kirchheim am Ries waren die Ätzbedingungen präzise festgelegt (vgl. 3.2.1.6, S. 49f). Die für diese Population dargestellten Daten resultieren aus jenem Probensatz des Versuches zur 
Probenaufreinigung der ohne Oberflächenbehandlung nur geätzt wurde (vgl. 2.2). Das Ergebnis ( $\mathrm{p}<0,001$, vgl. Tabelle 8) des statistischen Vergleichs für diese Population ist uneingeschränkt zu bewerten. Es wird festgestellt, daß schlecht erhaltene Knochenproben während des Ätzvorganges statistisch abgesichert mehr Masse verlieren als gut erhaltene Proben.

Tabelle 8: Vergleiche der Histologiegruppen hinsichtlich Masseverlust durch Ätzen (Mann-Whitney-U-Test)

\begin{tabular}{|l|c|c|c|c|}
\hline \multirow{2}{*}{\multicolumn{1}{|c|}{ Population }} & \multicolumn{2}{|c|}{ Gruppengrößen } & \multicolumn{2}{c|}{$\begin{array}{c}\text { Massenverlust- } \\
\text { Gruppenvergleiche }\end{array}$} \\
\cline { 2 - 5 } & $\begin{array}{c}\text { gute } \\
\text { Histologie }\end{array}$ & $\begin{array}{c}\text { schlechte } \\
\text { Histologie }\end{array}$ & Z-Wert & p-Wert \\
\hline Wenigumstadt & 29 & 57 & $(-4,75)$ & $\left(<0,001^{+}\right)$ \\
Weingarten & 25 & 38 & $(-4,79)$ & $\left(<0,001^{+}\right)$ \\
Kirchheim am Ries & 18 & 30 & $-4,98$ & $<0,001^{+}$ \\
\hline
\end{tabular}

${ }^{+}$bei festgelegtem $\alpha=0,05$ signifikant

\section{Masseverlust beim Veraschen}

Da die Veraschungsparameter für die ausgewerteten Populationen (Wenigumstadt, Weingarten, Kirchheim am Ries) exakt definiert waren (vgl. 3.2.1.4. 47f), sind im Gegensatz zum vorangegangen Kontrollkriterium alle populationsinternen statistischen Vergleiche uneingeschränkt interpretierbar.

Die deskriptiven Vergleiche der Daten in Abbildung 19 lassen sehr deutliche Unterschiede zwischen den Histologiegruppen erkennen.

Für alle drei Skelettpopulationen unterstützen die statistischen Testergebnisse diese Beobachtung (für alle $\mathrm{p}<0,001$ ). Nach Tabelle 9 können daher unter Bezug auf Abbildung 19 für schlecht erhaltene Knochenproben generell geringere Veraschungsverluste angenommen werden. 
a)
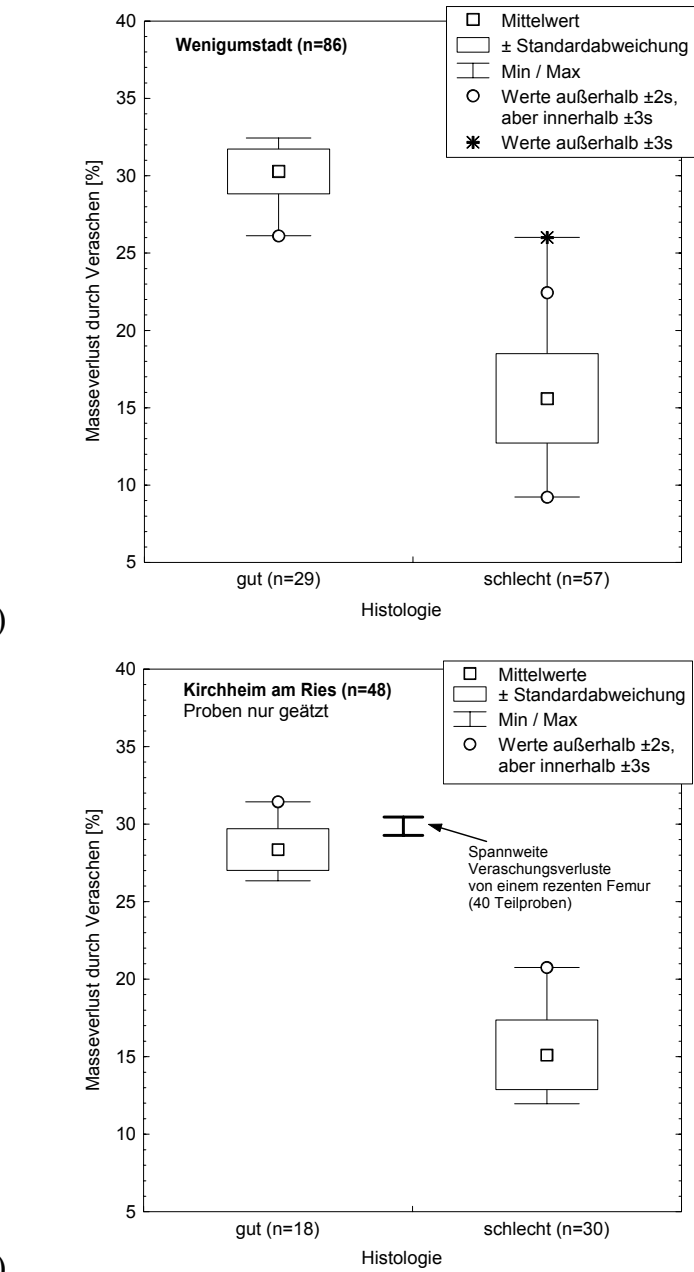

b)

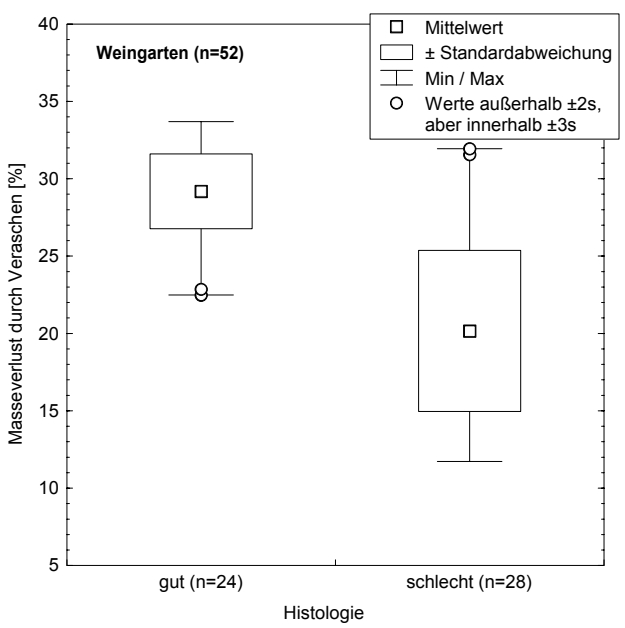

Abbildung 19: Statistisch deskriptiver Vergleich der durch das Veraschen bedingten Masseverluste zwischen den Histologiegruppen für a) Wenigumstadt, b) Weingarten und c) Kirchheim am Ries.

Die gleiche Skalierung in den drei Teilabbildungen ermöglicht den Vergleich zwischen den Populationen. Die in c) eingezeichnete Spannweite der Veraschungsverluste eines rezenten Femurs resultiert aus den Werten der 40 Teilproben der Untersuchung zur Erfassung der intraossären Konzentrationsvariabilitäten (vgl. Kapitel 4.5).

Tabelle 9: Vergleiche der Histologiegruppen hinsichtlich Veraschungsverlusten (Mann-Whitney-U-Test)

\begin{tabular}{|l|c|c|c|c|}
\hline \multirow{2}{*}{ Population } & \multicolumn{2}{|c|}{ Gruppengrößen } & \multicolumn{2}{c|}{$\begin{array}{c}\text { Massenverlust- } \\
\text { Gruppenvergleiche }\end{array}$} \\
\cline { 2 - 5 } & $\begin{array}{c}\text { gute } \\
\text { Histologie }\end{array}$ & $\begin{array}{c}\text { schlechte } \\
\text { Histologie }\end{array}$ & Z-Wert & p-Wert \\
\hline Wenigumstadt & 29 & 57 & 7,55 & $<0,001^{+}$ \\
Weingarten & 24 & 28 & 5,08 & $<0,001^{+}$ \\
Kirchheim am Ries & 18 & 30 & 5,75 & $<0,001^{+}$ \\
\hline
\end{tabular}

bei festgelegtem $\boldsymbol{\alpha}=\mathbf{0 , 0 5}$ signifikant

Durch die gleiche Skalierung der Teilgrafiken ist ein deskriptiver Interpopulationsvergleich direkt möglich. Für schlecht erhaltene Knochenproben ist eine Variabilität zwischen den verschiedenen Skelettkollektiven infolge unterschiedlich starker diagenetischer Beein- 
flussung erwartungsgemäß. Die gut erhaltenen Knochenproben von Kirchheim am Ries zeigen gegenüber den anderen Populationen bzw. im Vergleich zur rezenten Referenz etwas geringere Veraschungsverluste. Für Wenigumstadt und Weingarten sind die Veraschungsverluste der gut erhaltenen Knochenproben im Mittel mit rezentem Material vergleichbar. Aus Abbildung 19c ist zu entnehmen, daß der rezente Referenzwert von $30 \%$ auch von den im Rahmen dieser Untersuchung durchgeführten Analysen an einem rezenten Femur (vgl. 4.5) bestätigt wird.

\section{Zusammenfassung der Ergebnisse zu den diagenesecharakterisierenden Kontrollkriterien}

Für ursprünglich über das gute bzw. schlechte histologische Erhaltungsbild definierte Gruppen von Knochenproben wurden systematische Unterschiede der Konzentrationsverhältnisse (Ba/Ca- und Sr/Ca-Werte) festgestellt (vgl. 4.1, S. 80f). Daraus wurde geschlußfolgert, daß die Knochenproben entsprechend der unterschiedlichen Histologie verschieden stark diagenetisch veränderte sind.

Hiervon ausgehend wurden diese Histologiegruppen hinsichtlich sechs Kriterien verglichen, die den Erhaltungszustand der Knochensubstanz charakterisieren:

- Für die Barium-Strontium-Korrelation sowie das Calcium-Phosphor-Verhältnis konnten keine systematischen Unterschiede zwischen den Histologiegruppen gefunden werden.

- Der Vergleich der Magnesiumkonzentrationen ergab für gut erhaltene gegenüber den schlecht erhaltenen Knochenproben höhere Konzentrationen. Zusätzlich konnte für die Histologiegruppen ein markanter differenzieller Einfluß der säureinduzierten Aufreinigung festgestellt werden.

- Das Element Aluminium zeigte gegenüber Magnesium eine umgekehrte Charakteristik. Eine gute histologische Erhaltung ist mit geringen Aluminiumkonzentrationen verbunden, wohingegen die Proben mit schlechter Histologie höhere Gehalte dieses Elementes aufweisen.

- Sowohl für Magnesium als auch für Aluminimum entsprechen die Konzentrationsverhältnisse der gut erhaltenen Proben nicht denen nativem Knochenminerals. 
- Zwei spezifische Masseverluste während der Probenvorbereitung zeigten beim Vergleich der Histologiegruppen ebenfalls typische Muster. Schlecht erhaltene Knochenproben verlieren mehr Substanz durch den Ätzvorgang als gut erhaltene. Der Veraschungsvorgang hat hingegen bei schlecht erhaltenen Knochenproben geringere Masseverluste zur Folge als bei den Proben mit guter Histologie. 


\subsection{Ergebnisse der mikroanalytischen Untersuchungen eines Kompaktaquerschnittes}

In den vorangegangenen Ergebniskapiteln erfolgten pauschalchemische Auswertungen von Knochenkonzentrationen und Vergleiche zwischen Knochenproben. Im Folgenden werden Elementkonzentrationen innerhalb einer Knochenprobe mikrostrukturell differenziert dargestellt. Hierfür wurde der Kompaktaquerschnitt einer Probe mit einem histologischen Mosaik von gut und schlecht erhaltenen Kompaktaarealen analysiert. Es werden knochenintern Entsprechungen für die vorangegangenen probenvergleichenden Untersuchungen zur Charakterisierung diagenetischer Veränderungen der Knochensubstanz dokumentiert (vgl. 2.4).

\subsubsection{EPMA-Ergebnisse für die Elemente $\mathrm{Ca}, \mathrm{P}, \mathrm{Mg}, \mathrm{Na}$ und $\mathrm{S}$}

Im Kompaktaquerschnitt einer Knochenprobe werden innerhalb eines vom Periost bis zum Markraum reichenden Sektors (vgl. 2.4 bzw. 3.2.2.2, S. 54ff) Besonderheiten der Elementverteilungen dargestellt.

Für die Elemente Calcium, Phosphor und Magnesium sind diese Analysen in die Fragestellung zu den pauschalanalytischen Auswertungen in 4.3 (S. 92 bzw. 94) integriert. Natrium wurde erst zusätzlich in die detaillierten Auswertungen aufgenommen, nachdem sich aufschlußreiche und unerwartete Ergebnisse ergaben (vgl. 2.3, S. 30f). Da pauschalanalytische Ergebnisse für Schwefel mit der ICP-OES technisch nicht realisierbar waren, ist dieses Element unabhängig von den vorangegangenen Ergebnissen in diesen Untersuchungsteil eingebunden.

\section{Elementverteilungsbilder für $\mathrm{Ca}, \mathrm{P}, \mathrm{Mg}$ und $\mathrm{Na}$}

Die Elementverteilungen, d.h. die im Kompaktaquerschnitt variierenden Elementgehalte, wurden anhand der entsprechend unterschiedlichen Signalintensitäten der EPMA für das jeweilige Element ausgewertet (vgl. 3.2.2.2, S. 54ff). Diese Signalintensitäten werden in Abbildung 22 bzw. Abbildung 23) als verschiedene Farbwerte dargestellt (beachte: für Schwefel wurde kein Verteilungsbild erstellt, vgl. 3.2.2.2, S. 56).

Den Verteilungsbildern wird zunächst eine kurze Erläuterung zur dortigen Darstellung der Calcium-Phosphor-Verteilung sowie eine histologische Aufnahme (Abbildung 21) als "topographische" Orientierungshilfe vorangestellt. 
Da für die Elemente $\mathrm{Ca}$ und $\mathrm{P}$ deren Verhältnis $(\mathrm{Ca} / \mathrm{P}$, vgl. 4.3, S. 92ff) relevant ist, wird das Verteilungsmuster für beide Elemente zusammengefaßt als $\mathrm{Ca} / \mathrm{P}-\mathrm{Charakteristik} \mathrm{erfaßt.}$ Hierfür werden jedoch nicht numerische Werte des Ca/P-Quotienten in Farbewerte umgesetzt, sondern die Darstellung erfolgt entsprechend den Vorgaben in Abbildung 20:

Aus der bivariaten Auftragung der im periostnahen Teilbereich des analysierten Kompaktaausschnitts gemessenen Calcium- und Phosphor-Intensitäten resultiert eine als Konturenplot (Abbildung 20a) dargestellte Häufigkeitsverteilung (zur Erstellung der Elementverteilungsbilder vgl. 3.2.2.2, S. 55). Anhand dieser Häufigkeiten wurden über Wertebereichgrenzen für die Calcium- und Phosphor-Intensitäten "Phasen" definiert. Hierbei wurde der Intensitätsbereich mit der größten Häufigkeit als $\mathrm{Ca} / \mathrm{P}-H a u p t p h a s e($ gelb), dieser gegenüber höhere Absolutsignale als zweite Ca/P-Phase (rot) und niedrigere Absolutsignale als dritte Phase (grün) festgelegt. Diese "Phasen" unterscheiden sich hiernach nicht über die Werte des Ca/P-Quotienten, sondern über die Absolutsignale beider Elemente! Entsprechend dieser "Phasendefinition" wird die Ca/P-Charakteristik in den Verteilungsbildern dargestellt (Abbildung 22b bzw. Abbildung 23b).

a)

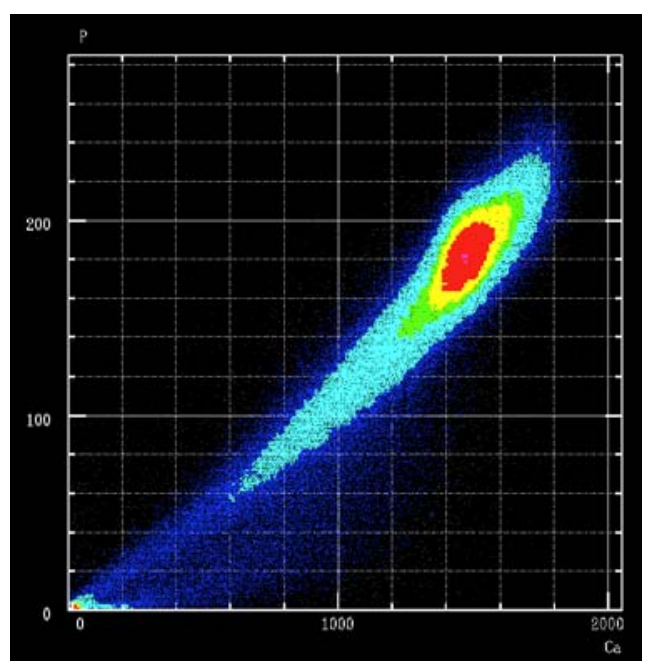

b)

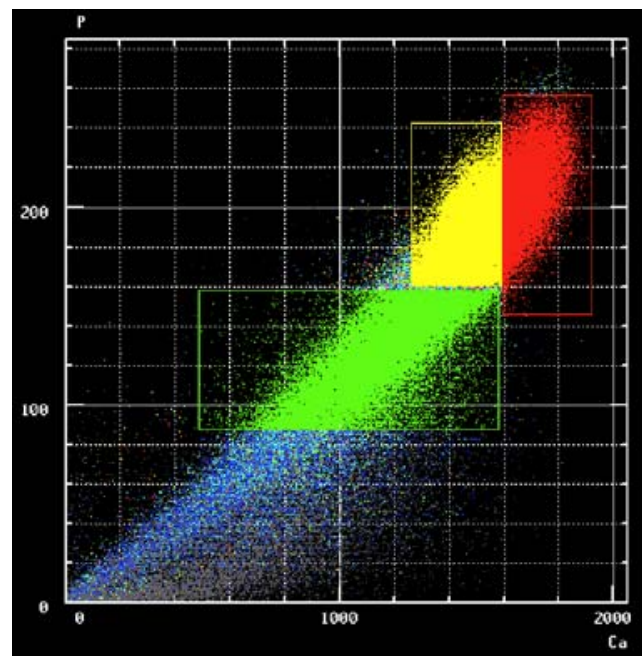

Abbildung 20: Ca- und P-Intensitäten (counts per step, als Maß für die jeweiligen Elementkonzentrationen) der Elementverteilungsanalyse mit der EPMA für den periostnahen Bereich des Kompaktaquerschnittes der Knochenprobe KR 261 von Kirchheim am Ries.

Dargestellt sind die Signalintensitäten aller $(920 \times 300)$ Meßpunkte des analysierten Areals (vgl. 3.2.2.2, S. 55). a) Häufigkeiten der Intensitätskombinationen als Konturenplot (Häufigkeitsgradient von zahlreich nach wenig: rot-gelb-grün-hellblau-dunkelblau) b) Definition der $\mathrm{Ca} / \mathrm{P}-\mathrm{Phasen}$ 
Abbildung 21 zeigt eine histologische Übersicht der untersuchten Knochenprobe (KR 261) von Kirchheim am Ries. Diese Abbildung dient zur Orientierung für die Verteilungsbilder. Die beiden Teildarstellungen sind von Periost (links) nach Markraum (rechts) orientiert. Teilabbildung a) zeigt den äußeren und Teilabbildung b) den direkt daran anschließenden inneren Bereich des Querschnittes. Da diese Aufnahmen von dem für die Probe hergestellten histologischen Präparat angefertigt wurden, zeigt der abgebildete Querschnittsektor nicht die gleiche Schnittebene, welche mit EPMA und LA-ICP-TOF-MS analysiert wurde. Die histologische Charakteristik in dieser lichtmikroskopisch erfaßten Schnittebene gleicht aber der in den SEM-Bildern von Abbildung 22a bzw. Abbildung 23a. Die "Topographie" des wechselnden Erhaltungsgrades der Knochenbinnenstruktur vom Periost zum Markraum (gut-schlecht-gut) findet in den SEM-Bildern von Abbildung 22a bzw. Abbildung 23a ihre Entsprechung. Hierfür ist Abbildung 21a Abbildung 22a bzw. Abbildung 21b Abbildung 23a zuzuordnen. Orientierung und Maßstab der Abbildungen stimmen überein.

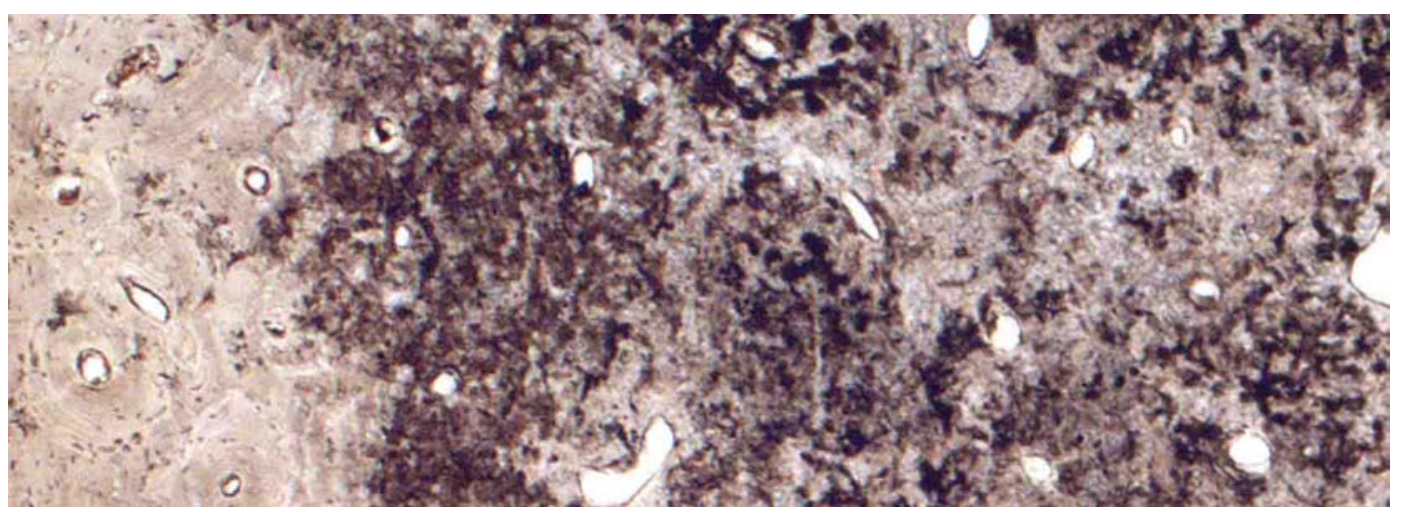

a)

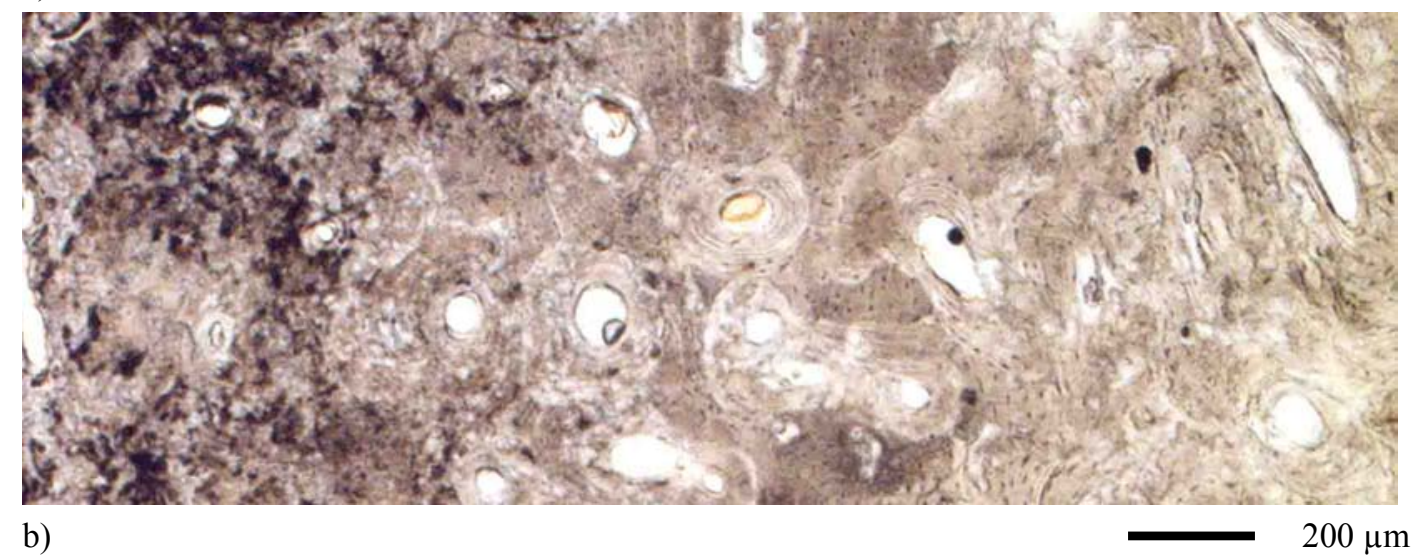

Abbildung 21: Lichtmikroskopische Aufnahmen eines Dünnschnittes (Transversalebene) der Probe KR 261 von Kirchheim am Ries. 


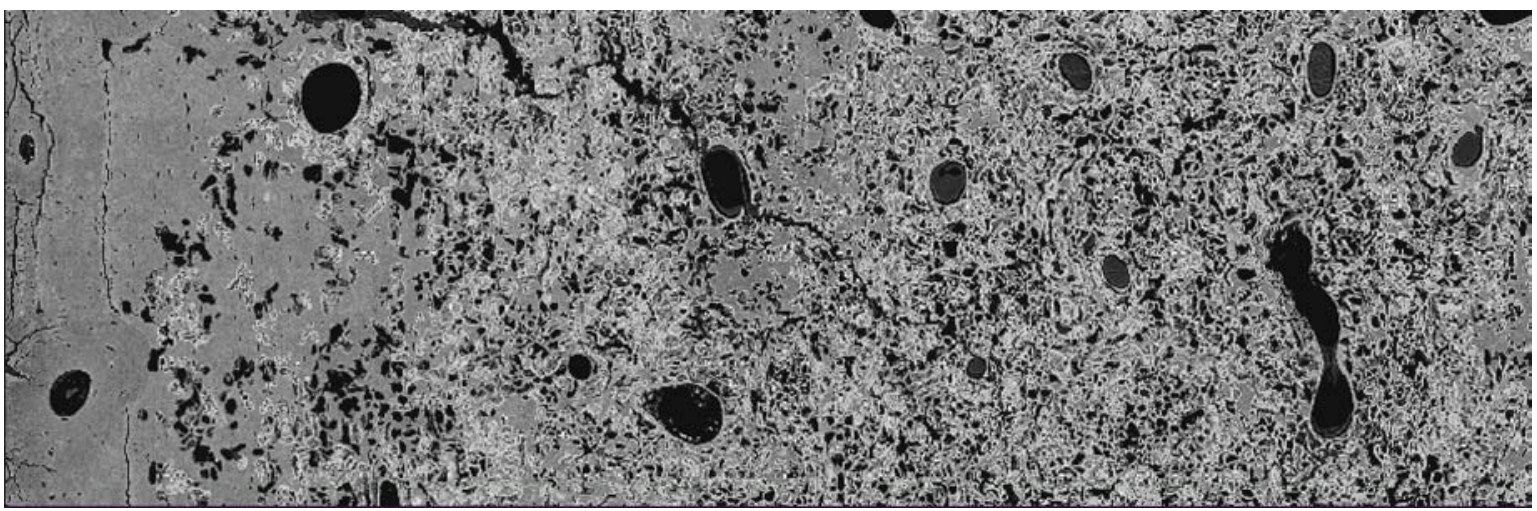

a)

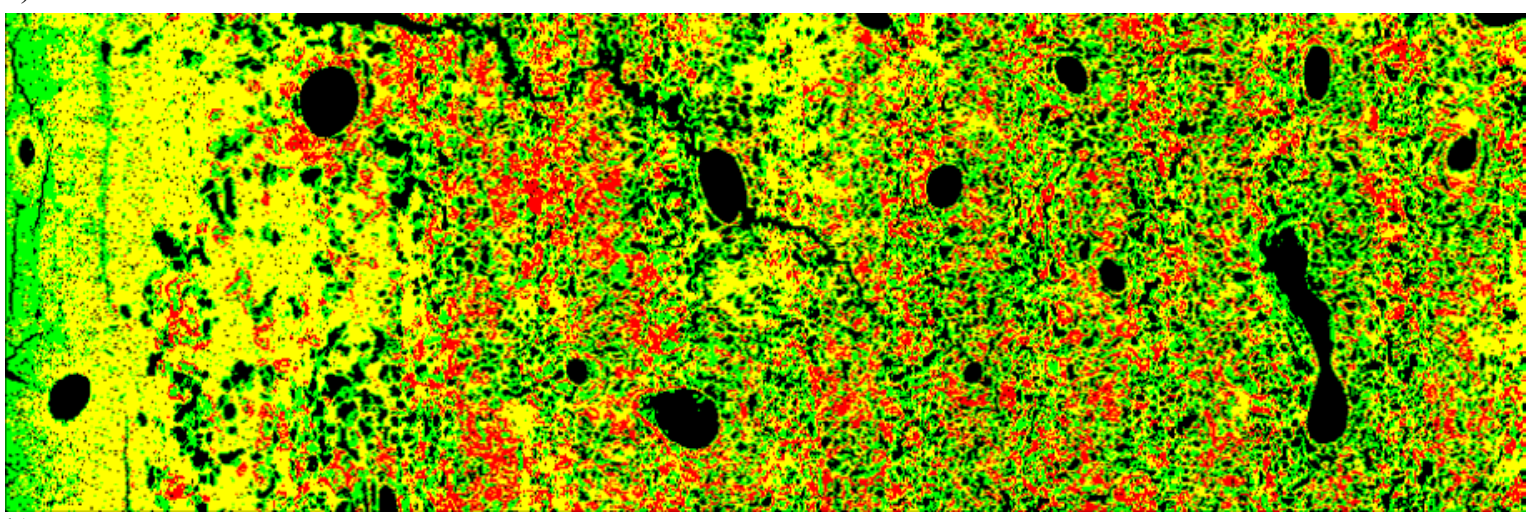

b)

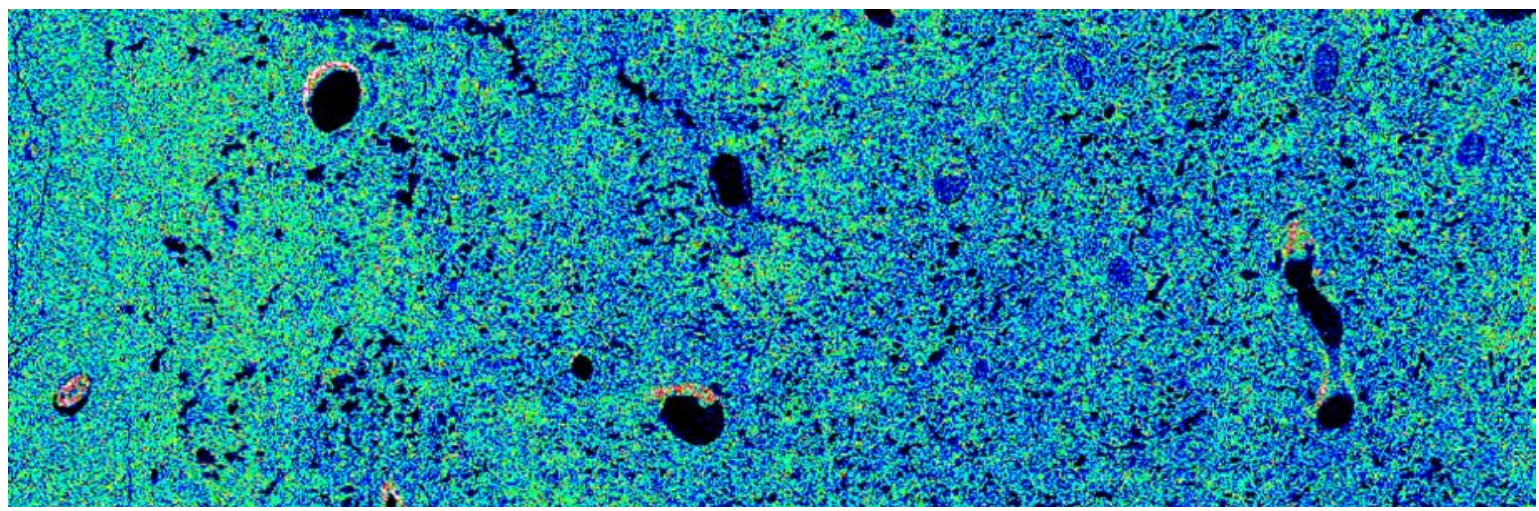

c)

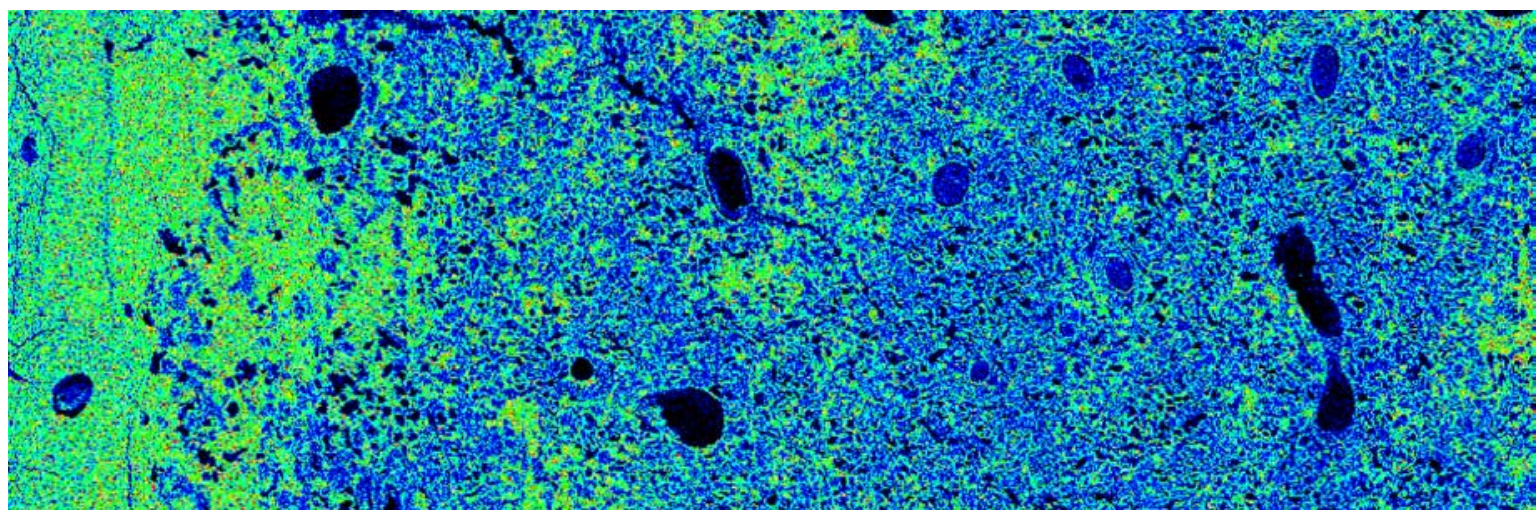

d)

$200 \mu \mathrm{m}$

Abbildung 22: Elementverteilungen im periostnahen Bereich des Kompaktaquerschnittes von KR 261.

Die Orientierung ist von Periost (links) nach Markraum (rechts): a) SEM-Aufnahme im Composition-Modus; b) Verteilung der in Abbildung 20 definierten Ca-P-Phasen; c) Mg-Verteilung; d) Na-Verteilung. In den Abbildungen $\mathrm{c}+\mathrm{d}$ entspricht der Farbübergang schwarz-violett-blau-grün-gelb-orange-rot dem Intensitätsgradienten der elementspezifischen Meßsignale (counts per step) von niedrig zu hoch (vgl. Text, S. 108 u. 111) 


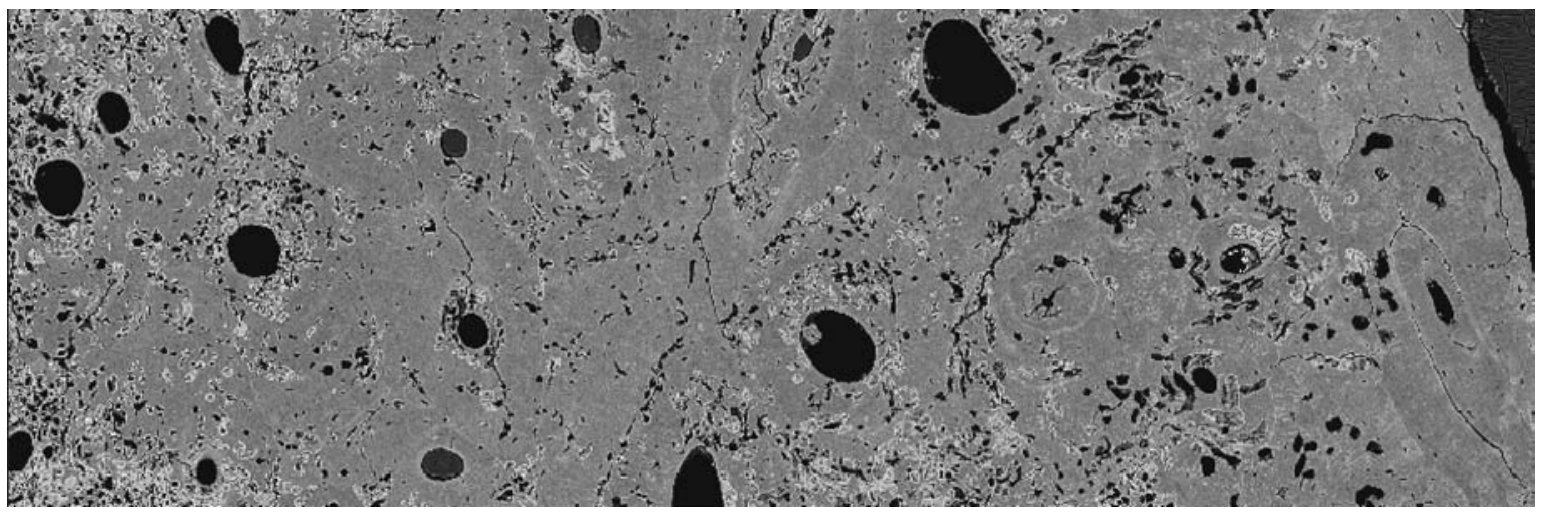

a)

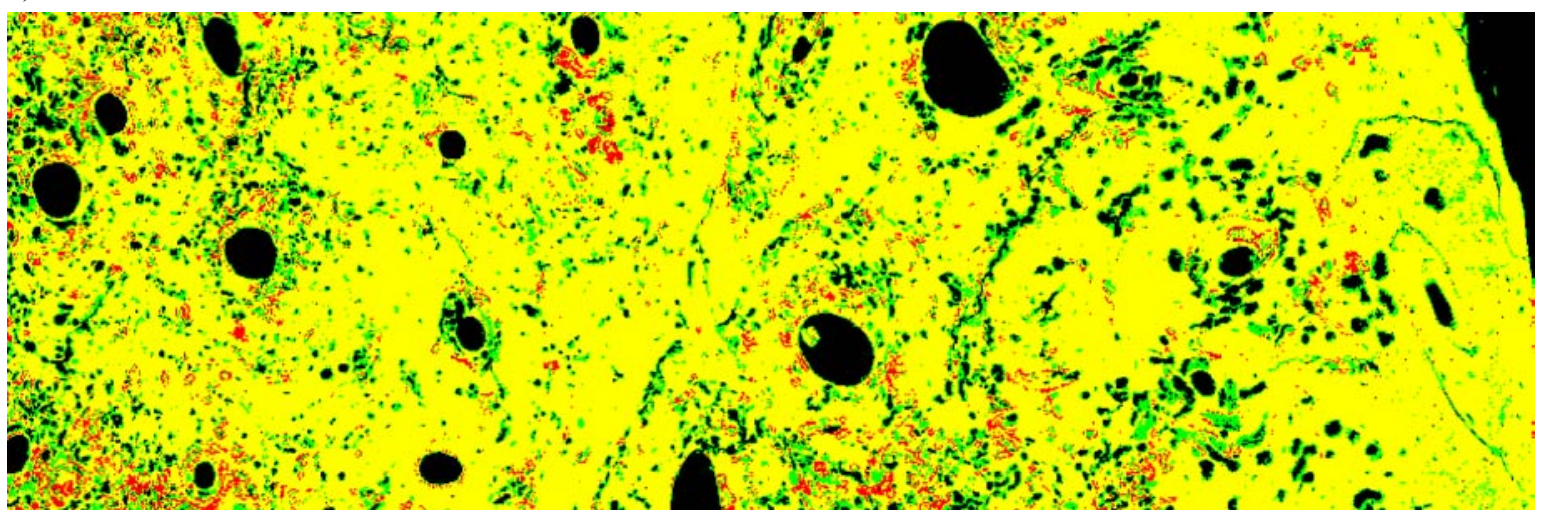

b)

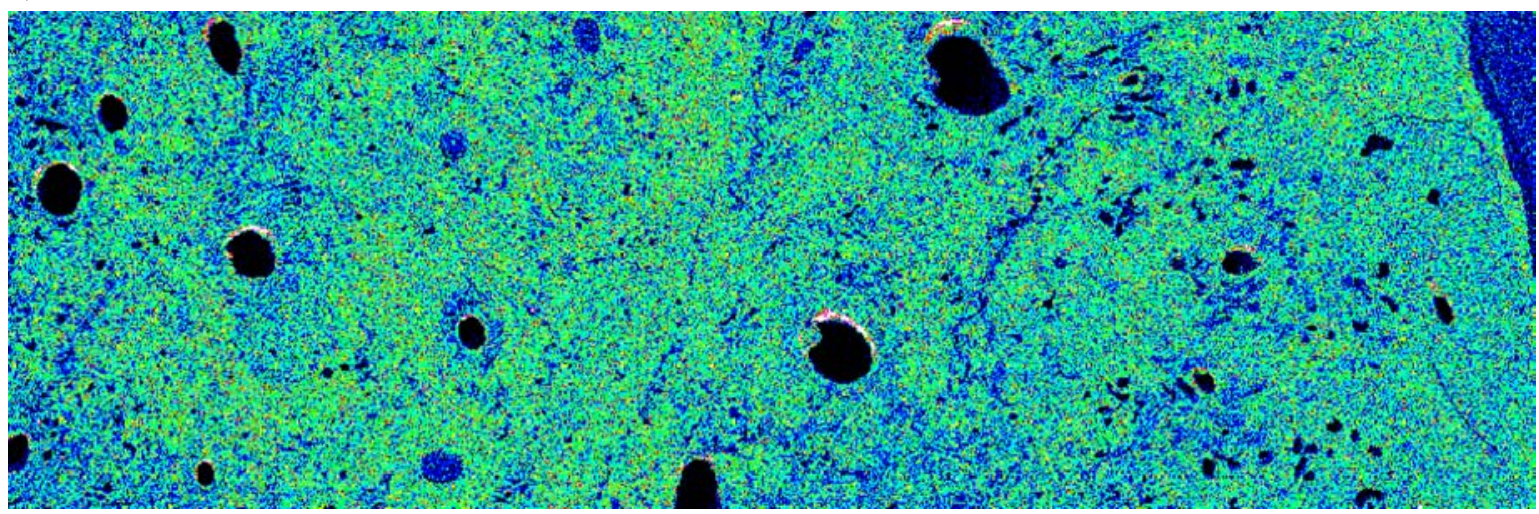

c)

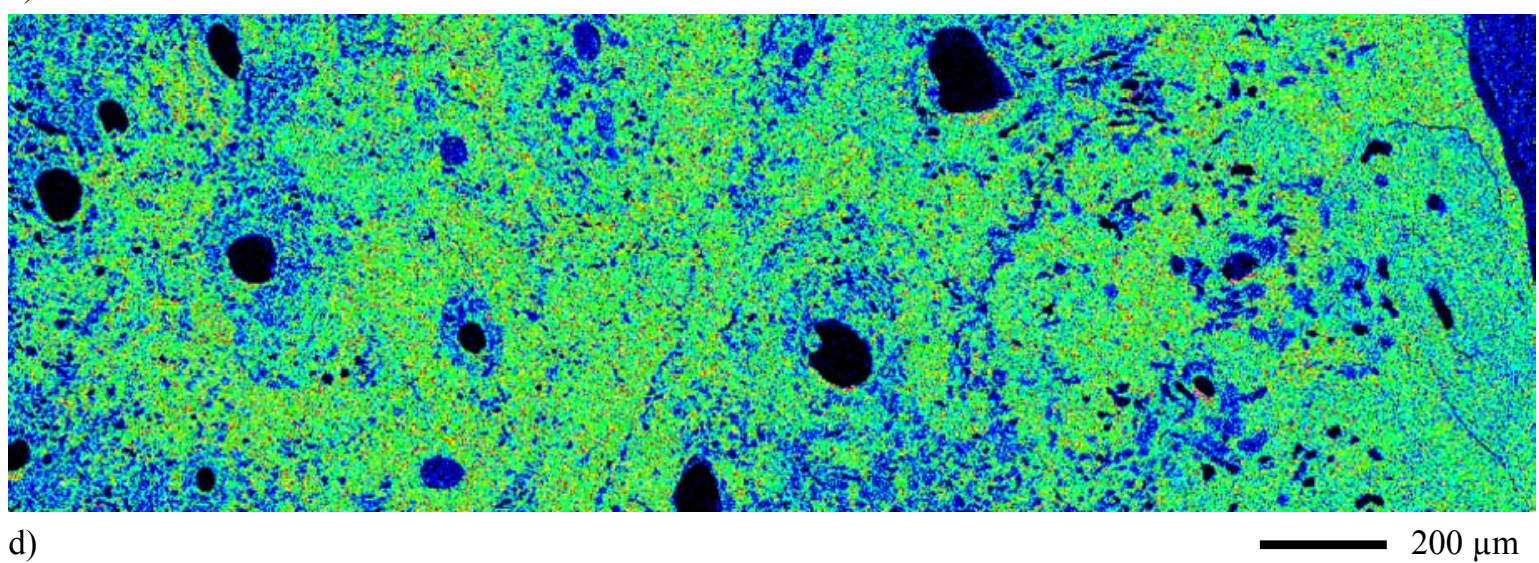

Abbildung 23: Elementverteilungen im markraumnahen Bereich des Kompaktaquerschnittes von KR 261.

Der gezeigte Bereich schließt mit gleicher Orientierung an das in Abbildung 22 dargestellte Areal an. Zuordnung der Teilabbildungen und Erklärung ist gleich der Abbildung 22 (vgl. Text, S. 108 u. 111). 
Die in Abbildung 21 histologisch als gut bzw. schlecht erhalten deklarierten Strukturbereiche innerhalb des Querschnittes werden in den SEM-Bildern (Abbildung 22a und Abbildung 23a) als "nativ" bzw. als veränderte Mineralareale angesprochen. Die Anführungsstriche bei "nativ" betonen den Vorbehalt, daß für diese Bereiche der native Zustand nur angenommen werden kann. Veränderte Mineralareale sind im SEM-Bild (Composition-Modus) als helle (weiße) durch dunkle, wahrscheinlich mineralfreie Gebiete gestörte Strukturen zu erkennen. Davon heben sich die relativ homogen grau erscheinenden "nativen" Bereiche ab. Im Überblick ergibt sich für die Abbildung 22a und Abbildung 23a gemeinsam folgende radiale Abfolge: Einem periostseitigen schmalen "nativen" Streifen folgt ein breiter veränderter Bereich. Dieser nimmt den Rest von Abbildung 22a zuzüglich den Beginn von Abbildung 23a ein. Dort schließt bis zum Markraum ein breiter Streifen großflächig "nativen" Minerals mit eingesprenkelten, veränderten Bereichen an.

Die Ca/P-Phasen sowie die Verteilungen der Elemente Magnesium und Natrium zeigen innerhalb dieses diagenetischen Mosaiks ein eindeutiges Muster.

"Native" Mineralareale sind durch die gelbe Ca/P-Hauptphase gekennzeichnet. Die durch proportional erhöhte (rot) oder verringerte (grün) Calcium- und Phosphorgehalte charakterisierten $\mathrm{Ca} / \mathrm{P}$-Phasen sind dagegen fast ausschließlich in den veränderten Kompaktabereichen lokalisiert (Abbildung 22b bzw. Abbildung 23b).

Die Elemente Magnesium (Abbildung 22c bzw. Abbildung 23c) und Natrium (Abbildung 22d bzw. Abbildung 23d) zeigen in den "nativen" Arealen gegenüber den veränderten Bereichen höhere Gehalte. Die Beobachtung größerer Flächenanteile mit einer calcium- bzw. phosphorabgereicherten grünen $\mathrm{Ca} / \mathrm{P}$-Phase innerhalb der veränderten Mineralareale erlaubt anhand dieser Verteilungsbilder aber keine eindeutigen Aussagen hinsichtlich der Konzentrationsrelationen dieser beiden Element in Bezug zum Matrixelement Calcium. Eine grüne $\mathrm{Ca} / \mathrm{P}$-Phase ist Indiz für eine insgesamt geringere Mineraldichte. Da für die Elemente Magnesium und Natrium in dieser Darstellung nur deren Absolutintensitäten abgebildet werden, kann ein geringerer Mineralgehalt geringere Absolutgehalte beider Elemente zur Folge haben, unabhängig von einer etwaigen An- oder Abreicherung gegenüber Calcium. Die calcium- bzw. phosphorreichen roten Areale (hauptsächlich in Abbildung 22b), die keine natrium- oder magnesiumangereicherte Entsprechung in Abbildung 22c $+\mathrm{d}$ finden, unterstützen aber die Annahme spezifisch geringerer Magnesium- und Natriumgehalte in veränderten Kompaktabereichen. Präzisiert werden diese Auswertungen durch die folgenden Ergebnisse der Einzelpunktanalysen mit quantifizierten 
Elementgehalten und der Berechnung der auf Calcium bezogenen Konzentrationsquotienten (s.u.).

Mit den genannten Einschränkungen zeigen die Verteilungsbilder eine Kongruenz zwischen mineralischer Erhaltung und Elementcharakteristik. Hierbei sind lokale An- bzw. Abreicherungsmuster evident.

\section{Einzelpunktanalysen der Konzentrationen von $\mathrm{Ca}, \mathrm{P}, \mathrm{Mg}$, Na und $\mathrm{S}$}

Die vorangegangenen Auswertungen der Elementverteilungsbilder des Kompaktaquerschnittes erfolgten über den relativen Vergleich von Signalintensitäten. Im Folgenden werden innerhalb dieses Kompaktaquerschnittes für Einzelpunktanalysen quantifizierte Konzentrationsdaten verglichen. Die Konzentrationen werden hierbei wieder als auf Calcium bezogene Quotienten $(\mathrm{Na} / \mathrm{Ca}, \mathrm{Mg} / \mathrm{Ca}, \mathrm{S} / \mathrm{Ca})$ ausgewertet. Calcium und Phosphor werden sowohl als Absolutkonzentrationen als auch als Ca/P-Quotient dargestellt.

Durch die präzise Anwahl von als "nativ" bzw. verändert angesprochenen Mineralarealen wurden zwei dem Erhaltungszustand entsprechende Meßpunktgruppen definiert, die anschließend statistisch verglichen wurden.

Die Meßpunktanwahl kann anhand Abbildung 24 nachvollzogen werden. In den SEMBildern (Composition-Modus) sind charakteristische Strukturen erfaßt. In Abbildung 24d (Detailvergrößerung von Abbildung 24c) sind "native" und veränderte Mineralbereiche gut unterscheidbar. Die Analysepunkte für die "native" Meßgruppe wurden in die grauen schwach "marmorierten" Areale gelegt (unter Ausschluß der helleren rißartigen Strukturen). Die Meßgruppe der veränderten Mineralareale setzt sich aus Analysepunkten in den porösen Formationen zusammen. Wie aus dem Maßstab in Abbildung 24d ersichtlich, werden solche Strukturen durch einen defokussierten Elektronenstrahl (5 $\mu \mathrm{m}$ Durchmesser, vgl. Anhang, Tabelle 16) möglicherweise als Ganzes erfaßt. In Abhängigkeit vom variablen Grad der Porosität können hieraus unterschiedliche Absolutkonzentrationen resultieren. Dies wird bei Magnesium, Natrium und Schwefel durch die Auswertung der calciumbezogenen Konzentrationsquotienten kompensiert (s.o.). 

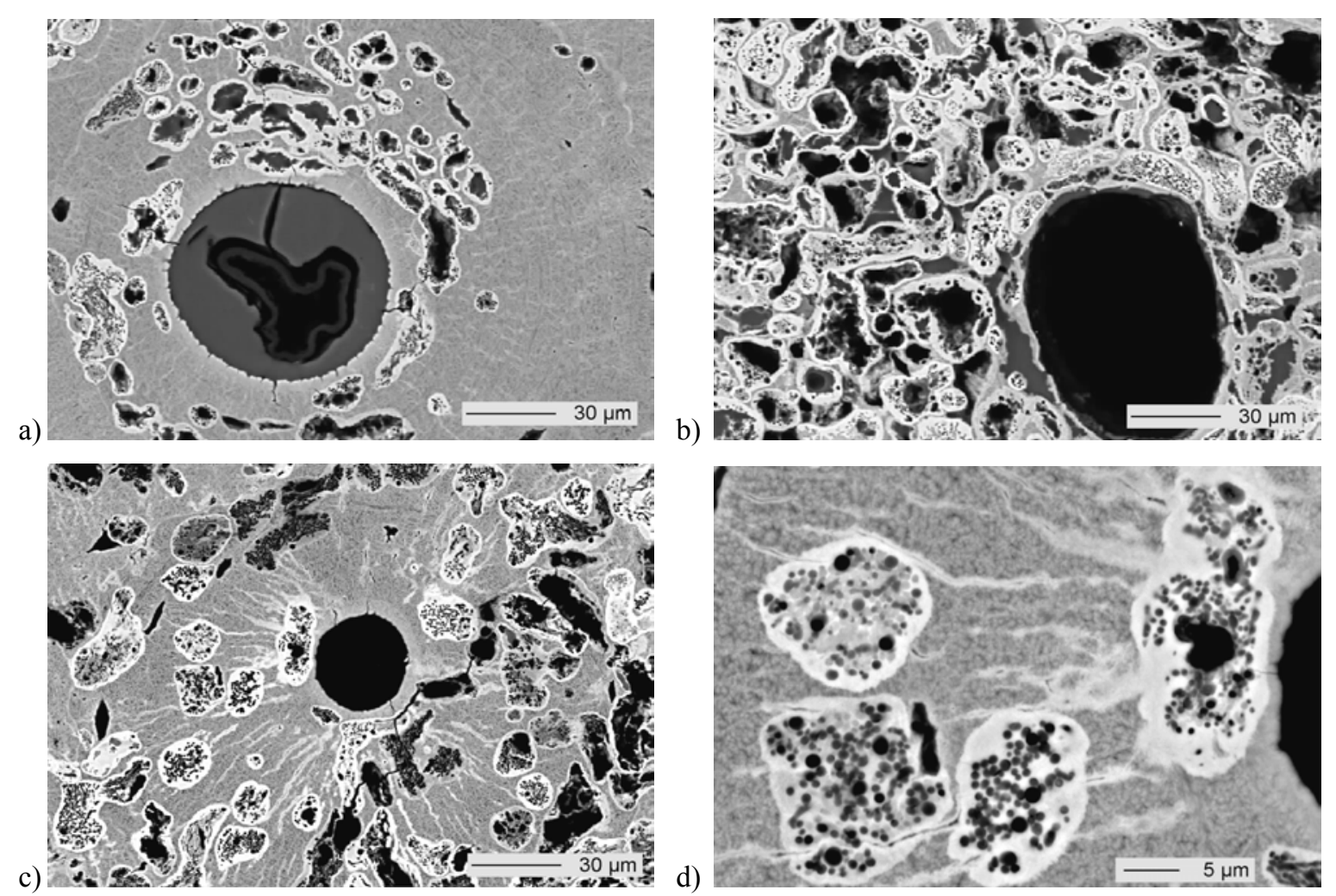

Abbildung 24: SEM-Detailaufnahmen im Composition-Modus vom Kompaktaquerschnitt der Knochenprobe KR 261.

Dargestellt ist jeweils der innere Bereich um den Haversschen Kanal eines Osteons. a) Beginn mineralischer Umbildung mit noch großflächigen "nativen" Mineralarealen; b) nahezu vollständige Umbildung des Minerals; c) intermediäres Stadium, zusätzlich zu den Umbildungsherden sind radial verlaufende veränderte Strukturen zu erkennen; d) Ausschnitt von c) zeigt, daß radiale Strukturen mit Rissen assoziiert sind.

Abbildung 25 zeigt die Ergebnisse dieser Einzelpunktanalysen für Calcium und Phosphor. Die hier gewählte Auftragung der Absolutkonzentrationen zeigt das aus den bisherigen Auswertungen bekannte "Ca/P-Phänomen" in besonders deutlicher Weise. Die Anordnung aller Datenpunkte entlang der dem Gesamtmittelwert aller Meßpunkte entsprechenden Geraden zeigt eine proportionale Charakteristik beider Elementgehalte unabhängig von der Lage der Meßpunkte in "nativen" oder veränderten Mineralarealen. Hiernach unterscheiden sich die beiden Erhaltungszustände des Knochenminerals nicht hinsichtlich der Relationen der Konzentrationen von Calcium und Phosphor. Der statistisch deskriptive Vergleich der $\mathrm{Ca} / \mathrm{P}-$ Werte zwischen der Meßgruppe der "nativen" Mineralareale und der veränderten Bereiche (eingefügte Grafik in Abbildung 25) deutet entsprechend nicht auf einen systematischen Unterschied. Ein solcher wird durch die statistische Prüfung (MannWhitney-U-Test) auch für unwahrscheinlich erklärt ( $\mathrm{p}=0,61$, vgl. Tabelle 10). 


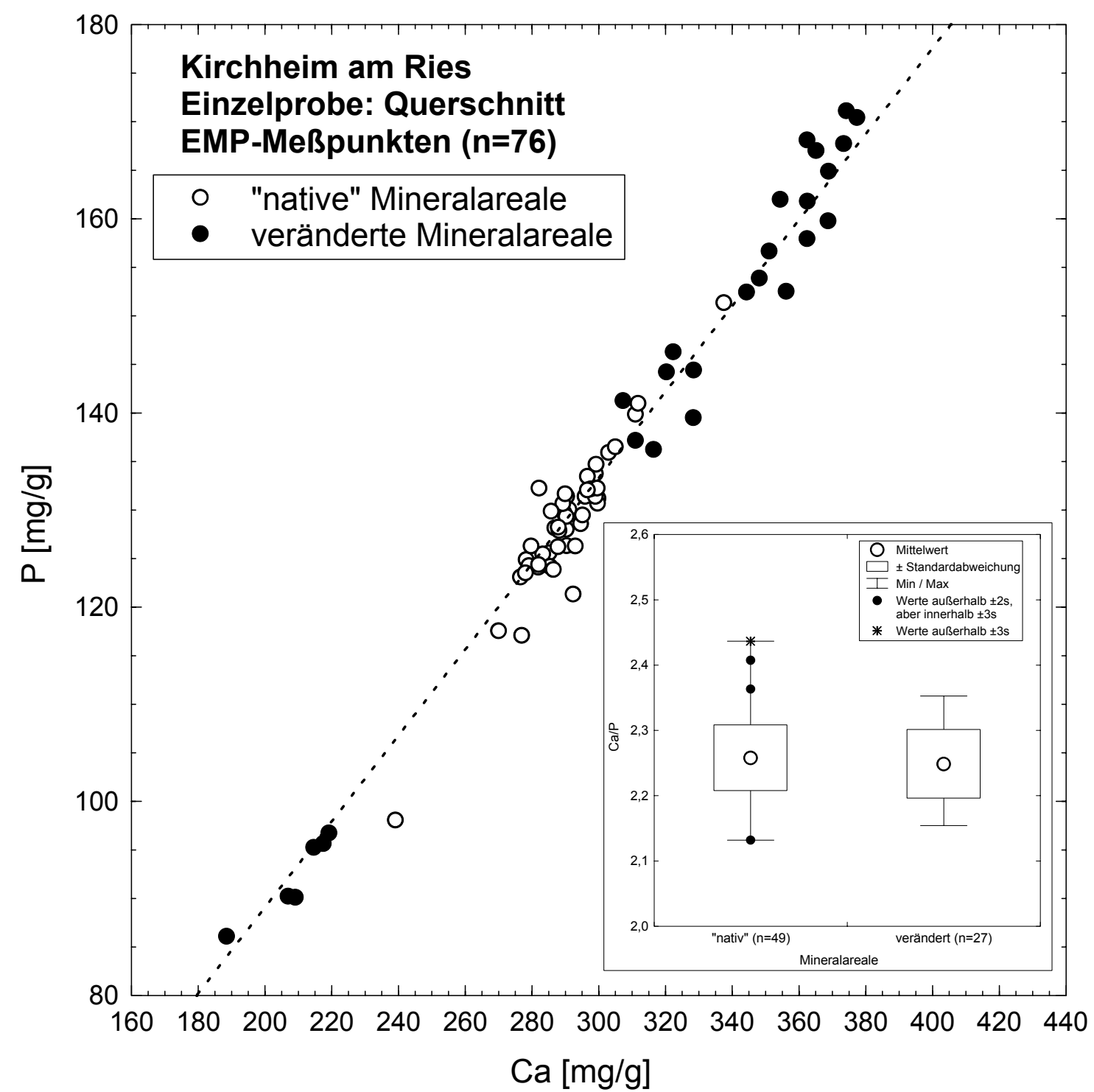

Abbildung 25: Ca- und P-Gehalte und resultierende Ca/P-Werte (eingefügte Grafik) der EPMA-Meßpunkte im Kompaktaquerschnitt.

$\mathrm{Zu}$ beachten ist, daß die Absolutgehalte aus den Gewichtsanteilen dieser Elemente bezogen auf Gesamtgewicht Knochen berechnet wurden und nicht auf Masse Knochenmineralasche bezogen sind. Die eingezeichnete gestrichelte Gerade entspricht dem mittleren $\mathrm{Ca} / \mathrm{P}$-Quotienten aller Meßpunkte.

Die nach Mineralerhaltung ("nativ" vs. verändert) kategorisierte Darstellung der Datenpunkte in Abbildung 25 zeigt jedoch charakteristische Unterschiede für die Absolutgehalte beider Elemente. Für die Meßpunkte in den veränderten Mineralarealen sind überwiegend höhere bzw. für wenige Meßpunkte geringere Absolutkonzentrationen beider Elemente zu beobachten. Sind niedere Absolutgehalte von Calcium und Phosphor durch das Problem der Porosität der analysierten Mineralareale möglicherweise artifiziell (vgl. S. 112), so sind die erhöhten Werte gerade durch diesen Umstand um so bedeutsamer zu bewerten. Dieses 
Ergebnis entspricht der mit der Phasendefinition in Abbildung 20 in den Verteilungsbildern der Abbildung 22b bzw. Abbildung 23b dargestellten Ca/P-Charakteristik.

In Abbildung 26 sind die Auswertungen der Einzelpunktanalysen hinsichtlich der Elemente Natrium, Magnesium und Schwefel zusammengefaßt.

a)

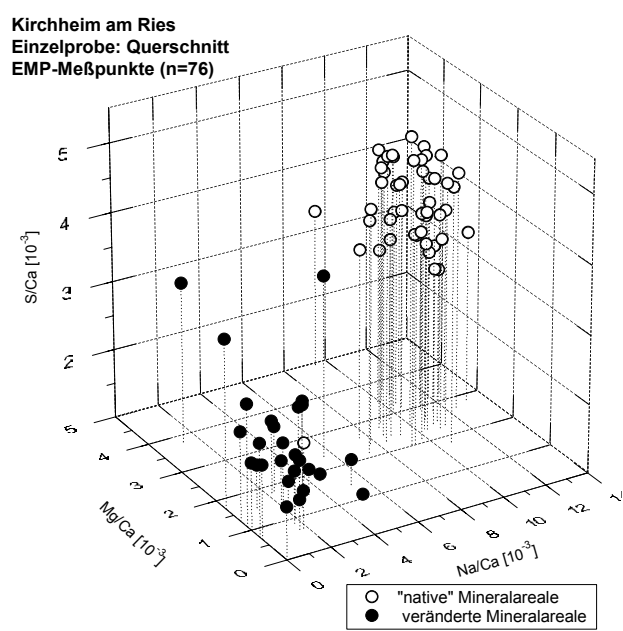

Abbildung 26: Na/Ca-, $\mathrm{Mg} / \mathrm{Ca}-$ und S/Ca-Werte sowie die Mineralsummen innerhalb eines Kompaktaquerschnittes mittels EMP bestimmt und nach knochenmineralisch "nativen" bzw. diagenetisch veränderten Mineralarealen kategorisiert.

a) Werteverteilungen der drei Konzentrationsquotienten. Die Kategoriesymbole sind entsprechend der bisherigen Histologiekategorisierung gut ( $\circ$ ) für "nativ" vs. schlecht (•) für verändert zugeordnet. Teilabbildung. b) Statistisch deskriptiver Vergleich zwischen "nativen" und veränderten Mineralarealen für $\mathrm{Na} / \mathrm{Ca}, \mathrm{Mg} / \mathrm{Ca}$ und $\mathrm{S} / \mathrm{Ca}$. Aus Absolutkonzentrationen unterhalb der Nachweisgrenze bei $\mathrm{Mg}$ und $\mathrm{Na}$ resultieren für die zugehörigen Konzentrationsquotienten Nullwerte, die folglich auch die Minima bilden.

Die Elementgehalte sind wieder als auf Calcium bezogene Konzentrationsquotienten dargestellt. In Abbildung 26a ist ein deutliches Zweigruppenmuster von Konzentrationsverhältnissen zu erkennen. Die kategorisierte Darstellung der Meßpunkte ("nativ" vs. verändert) zeigt, daß die beiden Konzentrationsgruppen den beiden nach Mineralerhaltung differenzierten Gruppen entsprechen. Für alle drei Elemente zeigen die Meßpunkte in "nativen" Mineralarealen gegenüber denen in veränderten Bereichen höhere Werte. Diese Gruppendivergenz wird in der statistisch deskriptiven Darstellung in Abbildung 26b bestätigt. Nach der Prüfung (Mann-Whitney-U-Test) sind diese Unterschiede für alle drei Elemente $(\mathrm{p}<0,001, \mathrm{vgl}$. Tabelle 10) auch statistisch relevant. 
Tabelle 10: Vergleich "nativer" und veränderter Mineralareale innerhalb eines Kompaktaquerschnittes bezüglich der $\mathrm{Ca} / \mathrm{P}-, \mathrm{Na} / \mathrm{Ca}-, \mathrm{Mg} / \mathrm{Ca}-$ und $\mathrm{S} / \mathrm{Ca}-W e r t e$ anhand der EPMA-Einzelmeßpunkte (Mann-Whitney-U-Test)

\begin{tabular}{|c|c|c|}
\hline \multirow{2}{*}{$\begin{array}{c}\text { Konzentrationsquo- } \\
\text { tient }\end{array}$} & \multicolumn{2}{|c|}{$\begin{array}{c}\text { Vergleich der Meßpunktgruppen } \\
\text { "native" (n=49) vs. veränderte }(n=27) \text { Mineralareale }\end{array}$} \\
\hline & Z-Wert & p-Wert \\
\hline $\mathrm{Ca} / \mathrm{P}$ & 0,52 & 0,606 \\
\hline $\mathrm{Mg} / \mathrm{Ca}$ & 6,47 & $<0,001^{+}$ \\
\hline $\mathrm{Na} / \mathrm{Ca}$ & 7,13 & $<0,001^{+}$ \\
\hline $\mathrm{S} / \mathrm{Ca}$ & 6,75 & $<0,001^{+}$ \\
\hline
\end{tabular}

${ }^{+}$bei festgelegtem $\boldsymbol{\alpha}=\mathbf{0 , 0 5}$ signifikant

Zusammengefaßt bestätigen die Ergebnisse der Einzelpunktanalysen im Kompaktaquerschnitt für Magnesium und Natrium die Ergebnisse der Verteilungsbilder (vgl. Abbildung 22c+d bzw. Abbildung 23c+d). Für Schwefel wird ein analoges Muster höherer Konzentrationen in den "nativen" Mineralarealen festgestellt.

\subsubsection{LA-ICP-TOFMS-Ergebnisse der Elemente $\mathrm{Ba}, \mathrm{Sr}$ und $\mathrm{U}$}

Für die drei weiteren untersuchungsrelevanten Elemente Barium, Strontium und Uran konnten die Analysen innerhalb des Kompaktaquerschnittes nicht mit der EPMA realisiert werden, da die Knochengehalte dieser Elemente zu gering waren. Die entsprechenden Analysen wurden daher mit LA-ICP-TOFMS durchgeführt.

Die Laserablationen wurden mit einem Durchmesser von $120 \mu \mathrm{m}$ (vgl. Anhang, Tabelle 18) durchgeführt. Eine der EPMA vergleichbare "präzise Zielansprache" ist daher nicht möglich gewesen. Wie in Abbildung 27a erkennbar, wurde eine radial verlaufende Meßpunktreihe analysiert.

Da aber die Ablationsanalysen innerhalb desselben Kompaktasektors erfolgten, der mit der EPMA analysiert wurde (vgl. 3.2.2.2, S. 56f), konnte eine nachträglichen Zuordnung der Ablationsorte in den SEM-Bildern durchgeführt werden. In Abbildung 27b1+b2 sind daher die SEM-Bilder der EPMA-Untersuchungen integriert (vgl. Abbildung 22a bzw. Abbildung 23a). Abbildung 27a zeigt eine nach den Ablationen erstellte Aufnahme des Kompaktaquerschnittes. Die Zuordnung der hier als weiße Punkte markierten Laserablationsorte ist durch die Pfeile und Kreise in Abbildung 27a+b kenntlich gemacht. Innerhalb der markierten Kreise konnten somit die Anteile "nativen" bzw. veränderten Minerals der 
Meßpunkte abgeschätzt werden. Hiervon ausgenommen sind die Meßpunkte 1, 20, 22 und 23, die außerhalb des mit der EPMA erfaßten Bereiches lagen.

Die Auswertung der massenspektrometrischen elementspezifischen Signale der Elemente Barium, Strontium und Uran erfolgte als auf das zugehörige Calciumsignal bezogene Signalquotienten (vgl. 3.2.3.1, S. 61). Bei Uran lagen die Signale von sechs Meßpunkten unterhalb der für Uran gesetzten Nachweisgrenze. Für diese Meßpunkte resultierten Nullwerte des U/Ca-Quotienten (vgl. 3.2.3.2, S. 65). In diesem Zusammenhang wird darauf hingewiesen, daß im Rahmen der LA-ICP-TOFMS-Analysen ebenfalls zunächst die Meßsignale für Aluminium ausgewertet wurden. Da die Aluminiumsignale der Meßpunkte innerhalb der Knochenkompakta aber alle unterhalb des für Aluminium im Einbettmedium detektierten Signals lagen, wurde Aluminium von einer differenzierten Auswertung ausgeschlossen.

Auswertungsrelevant waren ausschließlich Unterschiede der Elementgehalte zwischen den Meßpunkten und daraus möglicherweise resultierende elementspezifische radiale Gradienten. In Abbildung 27c sind daher nur die standardisierten (z-transformierten) Werte der Signalquotienten dargestellt. Relationen zwischen den drei Konzentrationsquotienten sind hiermit also nicht erfaßt. Die dortigen Meßdaten sind wiederum durch Pfeile den Ablationsorten im Kompaktaquerschnitt zugeordnet.

Für das knochenfremde Element Uran ist in Abbildung 27c ein radialer Gradient zu erkennen. Vom Periost nehmen die Werte zum Kompaktainneren ab und zeigen in Markraumnähe einen sehr schwachen Anstieg.

Prinzipiell folgen die $\mathrm{Ba} / \mathrm{Ca}$ - und $\mathrm{Sr} / \mathrm{Ca}-\mathrm{Signalquotienten} \mathrm{dem} \mathrm{Verlauf} \mathrm{der} \mathrm{U/Ca-Werte.}$ Nach einem kleinen lokalen Maximum (Meßpunkte 22, 23, 24) werden die Signale zum Markraum hin aber nicht wie die für Uran wieder größer. Die Gradienten von $\mathrm{Ba} / \mathrm{Ca}$ - und $\mathrm{Sr} / \mathrm{Ca}-W e r t e$ sind außerdem deutlich ungleichmäßiger als der von U/Ca. Andererseits sind beide Werteschwankungen eng gekoppelt. Die Auswertung dieser gleichlaufenden Signalunterschiede zwischen benachbarten Ablationspunkten erfolgte mit Hilfe der Meßpunktzuordnung in den SEM-Bildern der EPMA. Für auffällige Signalunterschiede in Abbildung 27c sind in Abbildung 27b1+b2 die den Ablationsorten in Abbildung 27a zugehörigen Mineralareale in den SEM-Bildern markiert. Für die ausgewählten Meßpunktvergleiche wird deutlich, daß jeweils höhere $\mathrm{Ba} / \mathrm{Ca}-$ bzw. Sr/Ca-Werte für jenen Ablationsort mit einem 
größeren Anteil veränderter Mineralstruktur im Vergleich zu einem direkt benachbarten mit vermehrt "nativen" Mineralarealen bestimmt wurden.

Für die eingezeichneten Meßpunkte sind folgenden Übergangspaare hervorzuheben, wobei die in den Klammern angegebene Zuordnung ("nativ" vs. verändert) relativ zum jeweils anderen Meßpunkt ist (vgl. Abbildung 27b1+b2). Die angegebene Signalveränderung bezieht sich auf die $\mathrm{Ba} / \mathrm{Ca}$ - und die $\mathrm{Sr} / \mathrm{Ca}-\mathrm{Signalquotienten}$ gleichermaßen:

- von Meßpunkt 2 ("nativ") $\quad$ zu Meßpunkt 3 (verändert) : Signalanstieg

- von Meßpunkt 3 (verändert) zu Meßpunkt 4 ("nativ") : Signalabfall

- von Meßpunkt 6 ("nativ") zu Meßpunkt 7 (verändert) : Signalanstieg

- von Meßpunkt 8 (verändert) zu Meßpunkt 9 ("nativ") : Signalabfall

- von Meßpunkt 9 ("nativ") zu Meßpunkt 10 (verändert) : Signalanstieg

- von Meßpunkt 18 (verändert) zu Meßpunkt 19 ("nativ") : Signalabfall

- von Meßpunkt 24 (verändert) zu Meßpunkt 25 ("nativ") : Signalabfall

Desweiteren zeichnen sich die Meßpunkte 2 und 19 als fast vollständig "native" Mineralareale aus. Hierbei ist bemerkenswert, daß sich für Meßpunkt 19 zusammen mit den beiden direkt am Markraum lokalisierten Meßpunkten 28 und 29, die ebenfalls kaum veränderte Mineralstrukturen aufweisen, die geringsten $\mathrm{Ba} / \mathrm{Ca}-\mathrm{bzw}$. Sr/Ca-Werte im gesamten Radialverlauf des Kompaktaquerschnittes ergeben. Die beiden letztgenannten Meßpunkte stellen zusetztlich noch ein benachbartes Paar niedriger Signale (Plateau) dar.

Unabhängig von diesen in Abhängigkeit von der Mineralerhaltung charakteristischen Signaländerungen bzw. gleichbleibenden Signalen sind auch Änderungen zu beobachten, die nicht diesem Schema entsprechen. So ist z.B. der Signalabfall zwischen Meßpunkt 12 und 13 nicht eindeutig einer Verbesserung der Mineralerhaltung zuzuordnen. 

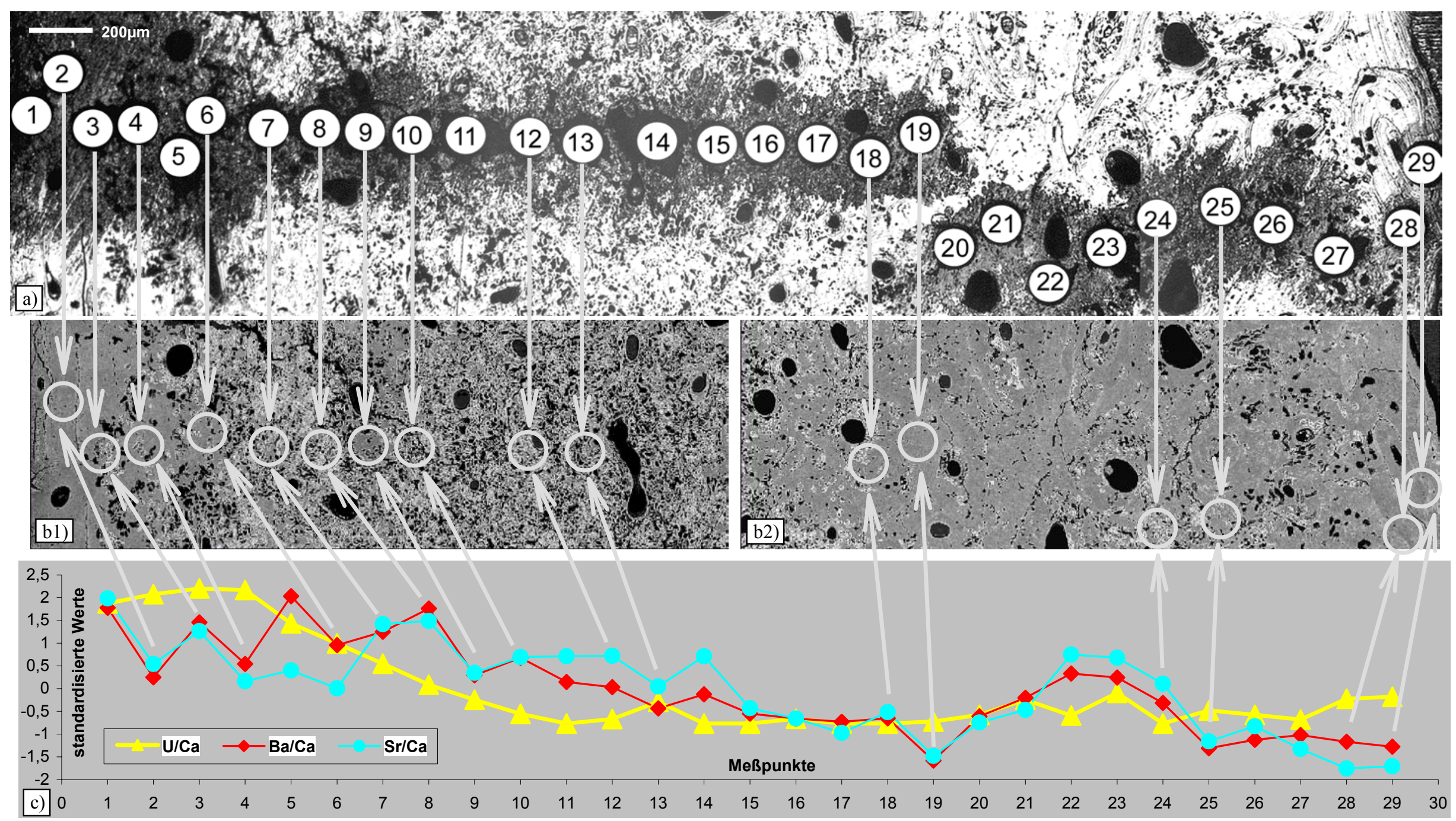

Abbildung 27: Auswertung der Meßpunkte der LA-ICP-TOFMS-Analyse.

Zur detaillierten Abbildungserläuterung siehe nächste Seite. 
Abbildung 27a): Meßpunkte der Laserablation (weiß markiert und nummeriert: 1-29) über den vollständigen Probenquerschnitt von Periost (links) nach Markraum (rechts) einer Knochenprobe von Kirchheim am Ries (KR 261). Die teilweise versetzten Meßpunkte resultieren aus dem Vermeiden von Haversschen Kanälen, Rißstrukturen bzw. sehr porösen und daher sehr mineralarmen Arealen.

b1+b2): SEM-Aufnahmen im Composition-Modus derselben Querschnittsektion wie unter a) vor der Laserablation im gleichen Maßstab. Diese beiden Teildarstellungen erfassen jenen Bildausschnitt von a), welcher ebenfalls für die EPMA-Elementverteilungsanalyse genutzt wurde (vgl. Abbildung 22a bzw. Abbildung 23a). Durch die Pfeile und Kreise werden die Meßpunkte der Laserablation in typischen Mineralarealen ("nativ" vs. verändert) gekennzeichnet. Diese Meßpunkte können wiederum in c) charakteristischen Elementgehalten bzw. -signalen zugeordnet werden (siehe Text).

c) Meßergebnisse der Laserablationsanalyse. Die Meßpunkte (1-29) entsprechen der Nummerierung aus a). Dargestellt sind die Veränderungen innerhalb des Querschnittes anhand der auf die Ca-Signale bezogenen U-, Ba- und Sr-Signale $\left({ }^{137} \mathrm{Ba}\right.$ und ${ }^{238} \mathrm{U}$ wurden als cps, ${ }^{88} \mathrm{Sr}$ und ${ }^{44} \mathrm{Ca}$ in $\mathrm{mV}$ registriert). Zur Vergleichbarkeit sind die berechneten Quotienten jeweils standardisiert (z-transformiert) dargestellt.

Auch wenn sehr hohe relative Fehler für Analysen mit LA-ICP-TOFMS zu beachten sind (30 \% vgl. 3.2.3.2, S. 64), werden die Beobachtungen charakteristischer Signalunterschiede zwischen Mineralarealen mit differentieller Erhaltung als systematisch eingeschätzt. Da aber keine hinreichende Quantifizierung des "nativen" bzw. veränderten Anteils der Mineralstruktur für die einzelnen Meßpunkte realisierbar war und damit keine Gruppeneinteilung der Meßpunkte erfolgen konnte, mußte auf einen statistischen Vergleich verzichtet werden.

Für die $\mathrm{Ba} / \mathrm{Ca}$ - und $\mathrm{Sr} / \mathrm{Ca}-W e r t e$ wird hiernach festgestellt, daß deren globaler abnehmender Gradient innerhalb der Kompakta von Periost zum Markraum durch lokale Unterschiede der Mineralerhaltung überzeichnet wird. Für veränderte Mineralareale werden höhere Gehalte für beide Elemente gegenüber direkt benachbarten "nativen" Bereichen beobachtet.

Der enge Zusammenhang der $\mathrm{Ba} / \mathrm{Ca}$ - und $\mathrm{Sr} / \mathrm{Ca}$-Signalquotienten innerhalb des Kompaktaquerschnittes wird über eine Korrelationsanalyse dokumentiert. Diese konnte infolge der nicht realisierbaren Gruppendifferenzierung in "native" vs. veränderte Meßpunkte (s.o.) nur für alle Meßpunkte gemeinsam erfolgen. In Abbildung 28 wird deutlich, daß unabhängig von den Unterschieden der Mineralerhaltung zwischen den einzelnen Meßpunkten ein starker Zusammenhang zwischen beiden Variablen für alle Meßpunkte gemeinsam zu beobachten ist. Die statistische Prüfung bestätigt dies mit einem hohen Korrelationskoeffizienten von $r=0,87$, der mit einer Irrtumswahrscheinlichkeit von $\mathrm{p}<0,001$ statistisch abgesichert ist. 
Für den analysierten Kompaktaquerschnitt wir festgestellt, daß bei kompaktaintern differentieller Erhaltung des Minerals eine mineralinterne (starke) Korrelation zwischen Barium- und Strontiumgehalten zu beobachten ist.

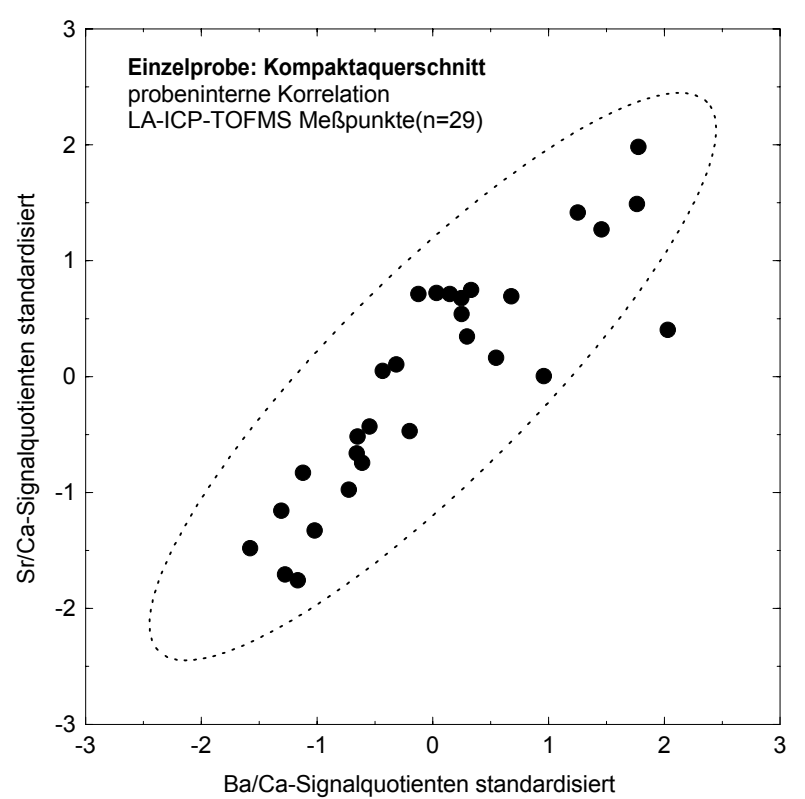

Abbildung 28: Korrelation der $\mathrm{Ba} / \mathrm{Ca}-$ und $\mathrm{Sr} / \mathrm{Ca}-$ Signalquotienten der LA-ICP-TOFMS-Analysen innerhalb des Kompaktaquerschnittes der Knochenprobe KR 261 von Kirchheim am Ries. Dargestellt sind die standardisierten Werte. Zusätzlich eingezeichnet ist die Konfidenzellipse mit einem Koeffizienten von 0,95. Durch diese wird die bivariate Verteilungscharakteristik sowie von dieser abweichende Einzelwerte veranschaulicht.

\subsubsection{Zusammenfassung der mikroanalytischen Ergebnisse}

Für die mikroanalytischen Untersuchungen werden im Folgenden die Ergebnisse zusammengefaßt. Die Reihenfolge der Ergebnisse entspricht dabei der Darstellung der Resultate der pauschalanalytischen Untersuchungen der diesen Ergebnissen vorangegangenen Kapitel 4.1 bis 4.3. Da die jeweiligen Teilergebnisse der mikroanalytischen Untersuchungen im Zusammenhang mit den pauschalanalytischen zu betrachten sind, wird auf die entsprechenden Kapitel bzw. relevanten Seitenzahlen verwiesen.

Innerhalb des Kompaktaquerschnittes der Knochenprobe KR 261 von Kirchheim Ries sind folgende elementanalytischen Charakteristika "nativer" bzw. veränderter Mineralareale festzustellen:

- Veränderte Mineralareale sind gegenüber "nativen" durch höhere Barium- und Strontiumgehalte gekennzeichnet (vgl. 4.1, S. 80 und 4.2, S. 82). Zusätzlich zu dieser Elementverteilungscharakteristik ist ein radialer Konzentrationsgradient zu beobachten. Von der Periostseite der Kompakta bis zum Markraum nehmen die Gehalte beider Elemente $a b$. 
- Unabhängig von der differentiellen Erhaltung des Knochenminerals und den damit variierenden Werten beider Signalquotienten zeigen die $\mathrm{Ba} / \mathrm{Ca}$ - und $\mathrm{Sr} / \mathrm{Ca}$-Signale eine starke positive Korrelation innerhalb des Kompaktaquerschnittes (vgl. 4.3, S. 88).

- Für das $\mathrm{Ca} / \mathrm{P}-$ Verhältnis konnten keine Unterschiede zwischen "nativen" und veränderten Mineralarealen festgestellt werden (vgl. 4.3, S. 92). Verändertes Knochenmineral ist jedoch durch proportional höhere Absolutgehalte von Calcium und Phosphor gekennzeichnet. Diese hypermineralisierten Strukturen (vgl. z.B. Pfeiffer \& Varney 2000) innerhalb der diagenetisch veränderten Kompaktabereiche sind gleichzeitig mit einer stärkeren Porosität verbunden. Aus diesem Effekt resultieren innerhalb dieser Bereiche lokal auch proportional verringerte Calcium und Phosphorgehalte.

- Magnesium, Natrium und Schwefel sind in "nativen" Mineralarealen in höheren Konzentrationen enthalten als in veränderten (zu Magnesium vgl. 4.3, S. 94)

- Uran als knochenfremdes Element zeigt einen radialen Konzentrationsverlauf innerhalb des Kompaktaquerschnittes mit einem schwachen U-förmigen Verlauf. Vom Periost in das Kompaktainnere werden die Konzentration zunächst geringer. In Markraumnähe sind wieder leicht erhöhte Gehalte vorhanden. 


\subsubsection{Nachtrag zum Element Na: Vergleich zwischen gut und schlecht erhaltenen Knochenproben in einer Stichprobe von Kirchheim am Ries}

Es wurde bereits in der vorangegangenen Zusammenfassung der mikroanalytischen Ergebnisse darauf hingewiesen, daß für die Elemente Schwefel und Natrium bei den pauschalanalytischen Untersuchungen keine vergleichbaren Ergebnisse zu Verfügung standen. Eine Konzentrationsbestimmung für Schwefel war weder mit der verfügbaren AAS- noch mit der ICP-OES-Technik realisierbar. Für Natrium wurden nach der Analyse charakteristischer Konzentrationsunterschiede zwischen "nativem" und verändertem Knochenmineral innerhalb einer Knochenprobe nachträglich entsprechende Analysen in einem begrenzten Umfang durchgeführt. Für eine Stichprobe von 10 Knochenproben (fünf histologisch gut erhaltene und fünf histologisch schlecht erhaltene Proben, vgl. 2.1, S. 12ff) der Skelettpopulation von Kirchheim am Ries wurden die Natriumkonzentrationen bestimmt.

Mit diesen Untersuchungen sollte exemplarisch geprüft werden, ob die Charakteristik der Unterschiede der Natriumkonzentrationen zwischen Knochenproben unterschiedlicher Erhaltung den mikroanalytischen Ergebnissen entspricht. Da die mikroanalytisch bestimmte Natriumverteilung in der Knochenkompakta jener für Magnesium analog ist (vgl. Abbildung 22c+d bzw. Abbildung 23c+d), erfolgte eine gezielte Vorauswahl der Proben. Es wurden histologisch gut erhaltene Proben mit sehr hohen bzw. schlecht erhaltene mit sehr niedrigen Magnesiumgehalten ausgewählt. Hierbei konnte auf die Konzentrationsdaten für diese Proben aus den Analysen zu 4.3 (S. 94) zurückgegriffen werden.

Die Ergebnisse dieser Natriumanalysen sind in Abbildung 29 zusammengefaßt. Es sind

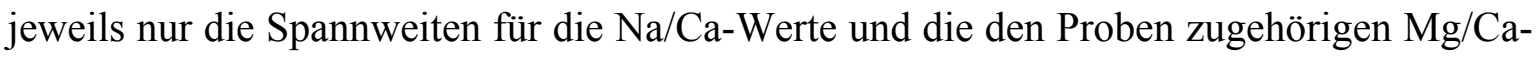
Werte der beiden Teilstichproben eingezeichnet. Es handelt sich hierbei um Konzentrationsdaten von Knochenproben, die zuvor sowohl oberflächenbehandelt als auch geätzt wurden. In der Abbildung sind zusätzlich die Angaben für acht Teilproben eines rezenten Femurs enthaltenen. Diese Natriumgehalte sind ebenfalls erst im Kontext dieses Untersuchungsteils in Teilproben des zur Erfassung der intraossären Konzentrationsvariabilität analysierten Femurs bestimmt worden (vgl. Folgekapitel 4.5). 


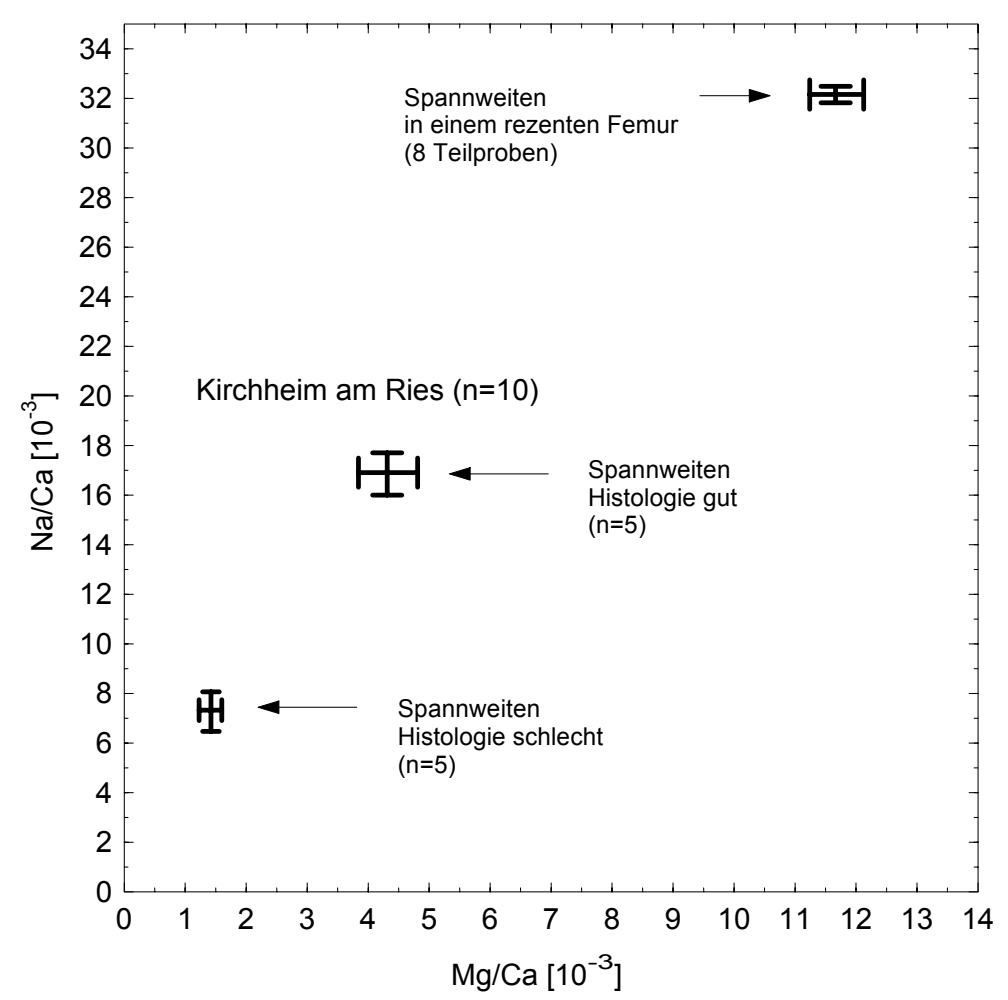

Abbildung 29: Vergleich der $\mathrm{Na} / \mathrm{Ca}-$ und $\mathrm{Mg} / \mathrm{Ca}-W$ erte zwischen Knochenproben mit guter bzw. schlechter histologischer Erhaltung von Kirchheim am Ries.

Dargestellt sind die aus den Einzelwerten resultierenden Spannweiten zweier Stichproben (je $\mathrm{n}=5)$.

Für diese Analysen sind gezielt gut erhaltene Proben mit bekannten, besonders hohen $\mathrm{Mg} / \mathrm{Ca}$ Werten bzw. schlecht erhaltene Proben mit besonders niedrigen $\mathrm{Mg} / \mathrm{Ca}-W e r t e n$ ausgewählt worden.

Zum Vergleich ist die Spannweite der $\mathrm{Na} / \mathrm{Ca}$-Werte von acht Einzelproben einer Knochenscheibe aus der Diaphysenmitte eines rezenten Femurs dargestellt.

Aus diesen Analysen ergibt sich für die Natriumgehalte der Knochenproben ein der Magnesiumcharakteristik analoges Bild. Natrium ist in histologisch gut erhaltenen Knochenproben in höheren Konzentrationen enthalten als in schlecht erhaltenen Proben. Auf einen statistischen Vergleich wird in Anbetracht der kleinen Stichprobe verzichtet. Als zweites Ergebnis ist hervorzuheben, daß, ebenfalls analog den Magnesiumergebnissen, auch die gut erhaltenen Knochenproben noch deutlich geringere Natriumgehalte als die rezente Referenz aufweisen (vgl. Ergebnisse zu Magnesium Abbildung 14)

Bleibt für Natrium abschließend festzustellen, daß diese pauschalanalytischen Vergleiche ganzer Knochenproben der mikroanalytischen Differenzierung zwischen "nativem" und verändertem Knochenmineral entsprechen (vgl. Abbildung 22d bzw. Abbildung 23d sowie Abbildung 26). 


\subsection{Intraossäre Variabilität der Sr- und Mg-Konzentrationen}

In die bisherigen Ergebnisse waren zu Vergleichszwecken wiederholt Daten eines rezenten Femurs integriert. Diese Daten resultieren aus der Untersuchung, die zur Erfassung der intraossären Variabilität von Spurenelementkonzentrationen durchgeführt wurde (vgl. 2.5). Primär orientierte die Fragestellung dieser Teiluntersuchung auf die Kontrolle der Ergebnisse des Probenaufreinigungsversuchs (vgl. 2.2). Nachdem nur marginale und damit unzureichende Aufreinigungseffekte festgestellt werden konnten (vgl. 4.2, S. 87), war ein Vergleich der durch die verschiedenen Aufreinigungsmethoden bedingten Konzentrationsdifferenzen mit der intraossären Konzentrationsvariabilität irrelevant.

Es wird aber im Folgenden ein Ergebnisaspekt der Analysen der rezenten Femurdiaphyse vorgestellt, der im Hinblick auf die Auswertung und Interpretation von Spurenelementkonzentrationen von Knochenproben zu diskutieren sein wird.

Für die 40 Teilproben (je acht Proben in fünf Horizonten unter Ausschluß des Bereichs der Linea aspera) des aus der Diaphysenmitte entnommenen Femurabschnittes (Länge des Abschnittes $10 \mathrm{~cm}$, vgl. 3.2.1.8) wurden die Elemente $\mathrm{Ca}, \mathrm{P}, \mathrm{Ba}, \mathrm{Sr}, \mathrm{Mg}$, $\mathrm{Al}$ und für den mittleren Diaphysenhorizont auch $\mathrm{Na}$ (vgl. vorangegangenes Kapitel) analysiert.

Die Daten für Calcium und Phosphor sind als $\mathrm{Ca} / \mathrm{P}$ mit bodengelagerten Knochenproben bereits verglichen worden (vgl. Abbildung 13). Auch die $\mathrm{Mg} / \mathrm{Ca}$-Werte wurden den entsprechenden Daten der Knochenproben von Kirchheim am Ries gegenübergestellt (vgl. Abbildung 14), werden aber in diesem Kapitel nochmals aufgegriffen (s.u.). Aluminium konnte erwartungsgemäß in allen 40 Teilproben der Femurdiaphyse nicht nachgewiesen werden (vgl. 2.3, S. 31). Dies bedeutet, daß dieses Element, wenn überhaupt, dann nur mit Konzentrationen kleiner als $10 \mu \mathrm{g} / \mathrm{g}$ im Knochen enthalten war. Barium konnte ebenfalls nicht nachgewiesen werden, d.h. die Knochenkonzentrationen dieses Elementes liegen unter $5 \mu \mathrm{g} / \mathrm{g}$ (zu den Nachweisgrenzen von Al und Ba vgl. Anhang, Tabelle 23, S. 165).

Als eigentlich auswertungsrelevant wurden aber die Charakteristika der Elementverteilung innerhalb der Femurdiaphyse für Strontium und zum Vergleich die für Magnesium erachtet. Anhand der $\mathrm{Sr} / \mathrm{Ca}-$ und $\mathrm{Mg} / \mathrm{Ca}-W e r t e$ werden diese in Abbildung $30 \mathrm{bzw}$. Abbildung 31 dargestellt. 


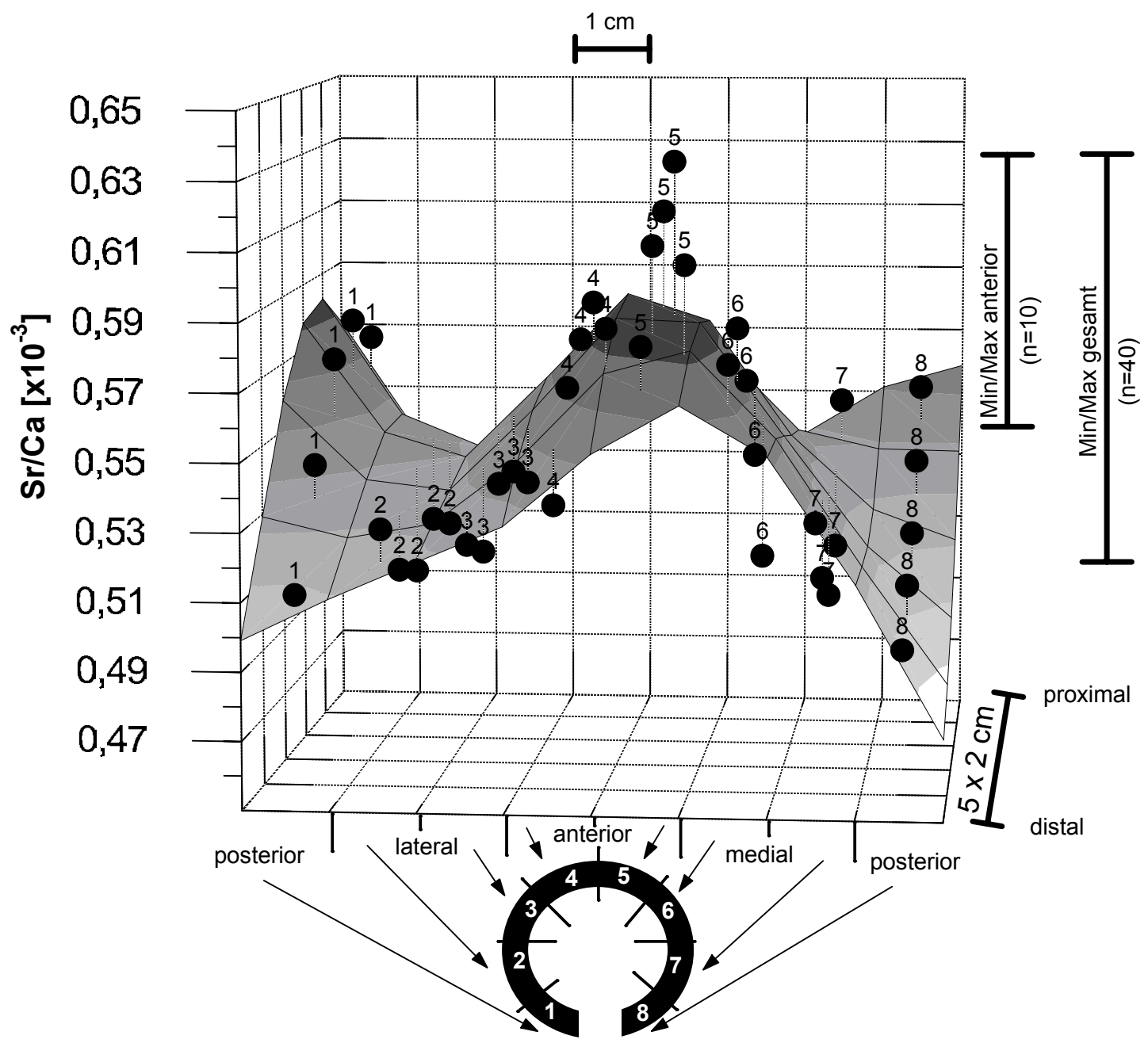

Abbildung 30: Sr/Ca-Werte-Verteilung innerhalb einer Femurdiaphyse (rechts, männlich, senil).

Erfaßt wurde ein Diaphysenausschnitt über eine longitudinale Ausdehnung von ca. $10 \mathrm{~cm}(5 \times 2 \mathrm{~cm})$. Jeder Datenpunkt resultiert aus der Pauschalanalyse einer Knochenprobe von ca. $1 \times 2 \mathrm{~cm}$ (horizonta-

le $\times$ longitudinale Ausdehnung). Es ergeben sich 40 Einzelproben (je Horizont die mit 1-8 bezeichneten acht Einzelproben).

Die Darstellung des Verteilungsmusters innerhalb des Femurschaftes durch die werteabhängig schattierten Flächen folgt einer Anpassungsfunktion (distanzgewichtete Kleinste-Quadrate-Glättung; Steifheit von 0,25; $9 \times 6$ Schnitte, StatSoft 1998).

Der erste Ergebnisaspekt bezieht sich für Strontium auf die Diaphysenregion, die dem standardisierten Entnahmeort für Proben bei bodengelagertem Knochenmaterial entspricht (vgl. 3.2.1.1). Für die Sr/Ca-Werte im anterioren Bereich der Diaphyse entspricht die in Abbildung 30 grafisch dargestellte absolute Spannweite $(0,09)$ 15,5\% des für diesen Bereich ermittelten Mittelwertes $(0,59)$. Diese Variabilität wird damit für einen Knochenstreifen von $2 \mathrm{~cm}$ Breite und $10 \mathrm{~cm}$ Länge innerhalb einer Diaphyse festgestellt. 
Als zweites Ergebnis wird eine sehr hohe Variabilität $(0,13)$ innerhalb des gesamten Diaphysenabschnittes festgestellt (vgl. in Abbildung 30 eingezeichnete absolute Gesamtspannweite). Dies bedeutet bei einem Gesamtmittelwert von 0,54 eine relative Variabilität von $24,1 \%$.

Im Zusammenhang mit Spurenelementuntersuchungen erlangt der dritte Auswertungsaspekt dieser Teiluntersuchung besondere Bedeutung. Innerhalb der analysierten Diaphysenhorizonte ist ein spezifisches Verteilungsmuster der $\mathrm{Sr} / \mathrm{Ca}-W e r t e$ zu beobachten (in Abbildung 30 durch die werteabhängig schattierte Anpassungsfläche dargestellt). Hierbei wird die stereotype Wiederkehr des Musters in jedem der acht Einzelhorizonte als Indiz seines systematischen Charakters gewertet. Im anterioren Femurbereich sind hiernach gegenüber den lateralen und medialen Bereichen höhere Werte festzustellen. Lateral folgen auf die niedrigen $\mathrm{Sr} / \mathrm{Ca}$-Werte nach posterior wieder deutlich höhere Konzentrationen. Medial ist der nach posterior zu beobachtende Konzentrationsanstieg weniger stark ausgeprägt. Nach diesen Ergebnissen ist Strontium im anterioren Bereich gegenüber den anderen Diaphysenarealen angereichert und zeigt keinesfalls eine zufällige (homogene) Verteilung.

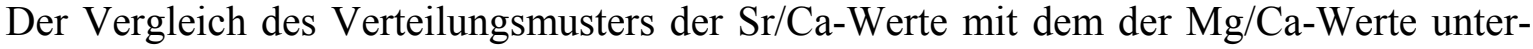
stützt die Hypothese systematischer Elementverteilungen in einer Femurdiaphyse. Die relative Gesamtvariabiltät innerhalb des Diaphysenabschnittes ist für die $\mathrm{Mg} / \mathrm{Ca}-\mathrm{Werte}$ mit 8,5\% geringer als die $\mathrm{Sr} / \mathrm{Ca}$-Variabilität. Unabhängig davon ist aber auch für Magnesium ein Verteilungsmuster zu erkennen: Bei diesem ist das laterale "Wertetal" jedoch deutlicher ausgeprägt als bei Strontium. Der medial-posteriore Abfall der $\mathrm{Sr} / \mathrm{Ca}-W e r t e$ ist bei den $\mathrm{Mg} / \mathrm{Ca}-$ Werten hingegen nahezu als Werteplateau nivelliert. 


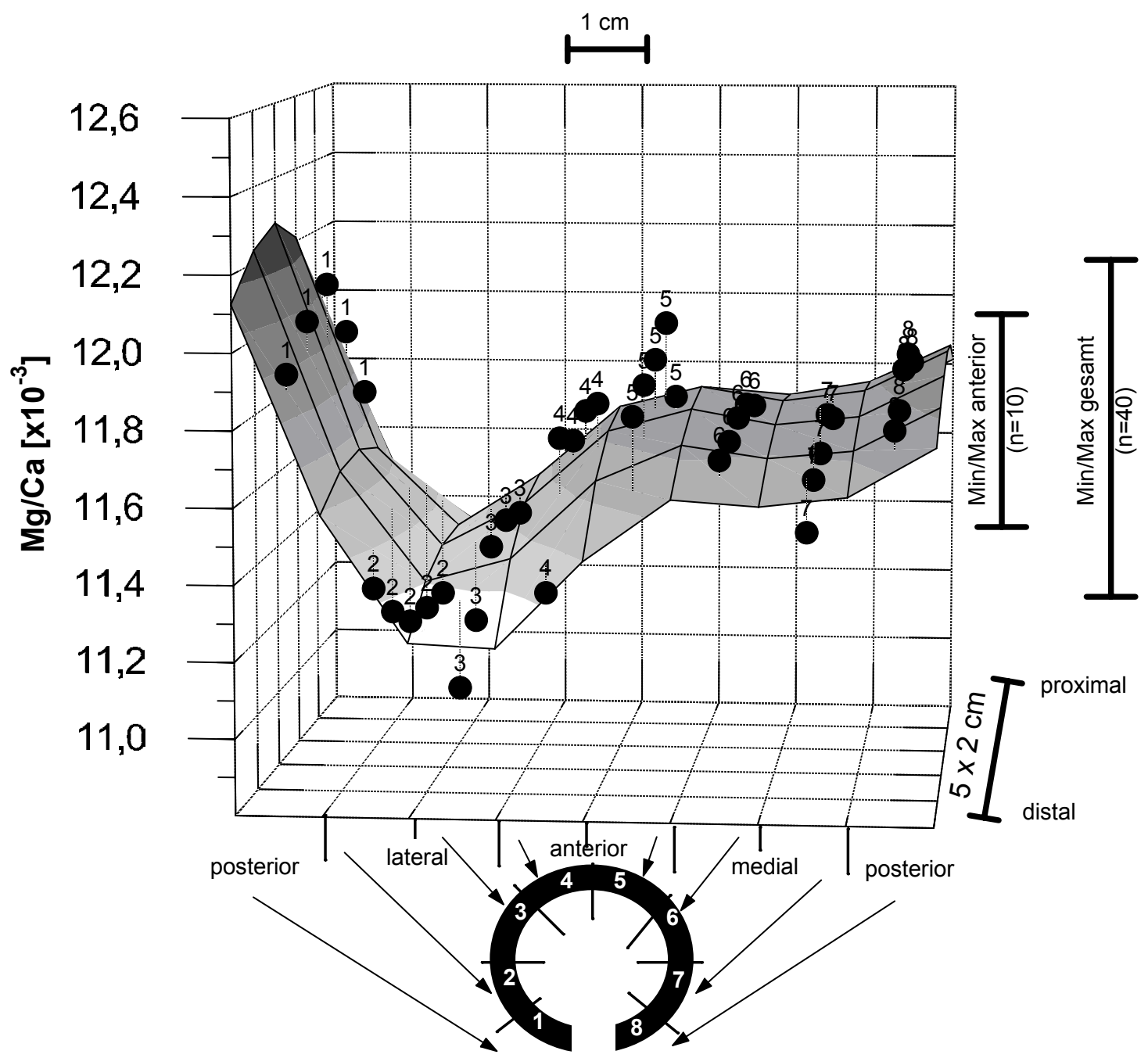

Abbildung 31: Mg/Ca-Werte-Verteilung innerhalb einer Femurdiaphyse (rechts, männlich, senil). Probenschema und Details entsprechen den Angaben zu Abbildung 30.

Nach diesen Ergebnissen zeigen die beiden Elemente Strontium und Magnesium differenzierte Muster ihrer Verteilung in einer Femurdiaphyse. Physiologische Einflüsse, die eine lokal variable Elementkomposition des Knochenminerals, einschließlich der Strontiumverteilung, zur Folge haben sind nach diesen Ergebnissen sehr wahrscheinlich. Eine mögliche physiologische Ursache des Verteilungsmusters für Strontium ist deshalb zu diskutieren (vgl. 5, S. 142).

Erkennbare longitudinale Verteilungsmuster beider Elemente sind für die acht Horizontareale uneinheitlich und dabei deutlich weniger prominent. Sie werden daher im Rahmen dieser Untersuchung nicht weiter ausgewertet. 


\section{$5 \quad$ Diskussion}

"If our knowledge of biochemistry of bone is incomplete, the geochemistry of soil-buried bone is virtually unknown." (Radosevich 1993, S. 312). Diese ernüchternde Feststellung verbunden mit der "präzisen" Beschreibung von Elliot (1994, S. 7): "A carbonate-containing OHAp-like salt forms the mineral of bones and teeth..." (OHAp = Hydroxylapatit) sollten $\mathrm{zu}$ bedenken geben, inwieweit diagenetische Veränderungen des Knochenminerals verstanden werden können.

Die Ergebnisse der vorliegenden Arbeit in diesen Forschungskontext einzubinden, beginnt daher mit einer Phänomenologie der im Knochen vorgefundenen Konzentrationsveränderungen.

\section{Die Evidenz, diagenetischer Veränderungen der $\mathrm{Ba} / \mathrm{Ca}$ - und $\mathrm{Sr} / \mathrm{Ca}$-Werte}

Wie in der Einleitung festgestellt, wurden und werden Barium- und Strontiumgehalte von bodengelagertem Skelettmaterial im Rahmen der Ernährungsrekonstruktion ausgewertet und interpretiert. Dies erfolgt unter Vorbehalt mit der Annahme, die (in Relation zu Calcium) bestimmten Knochengehalte repräsentierten in vivo generierte, ernährungsabhängige Konzentrationsverhältnisse (vgl. 1.2).

Faßt man die Ergebnisse dieser Arbeit zusammen, muß diese Interpretationsoption (vorsichtig eingeschränkt) für vier frühmittelalterliche Skelettserien als prinzipiell fraglich erklärt werden.

Ausgangspunkt für diese Feststellung ist die Gegenüberstellung früherer Interpretationen der Spurenelementdaten von Weingarten als Ernährungsgruppen mit dem in dieser Arbeit überprüften Erklärungsansatz einer diagenetischen Bedingtheit. Die Frage, welche der beiden Erklärungsvarianten (Ernährung vs. Diagenese) die populationsinterne Variabilität der $\mathrm{Ba} / \mathrm{Ca}$ - und $\mathrm{Sr} / \mathrm{Ca}-W e r t e$ beschreibt, ist eindeutig zu beantworten: $\mathrm{Die} \mathrm{Ba} / \mathrm{Ca}$ - und $\mathrm{Sr} / \mathrm{Ca}-$ Werte variieren ausschließlich in Abhängigkeit von der diagenetischen Veränderung des histologischen Erscheinungsbildes der Knochenproben. Der Versuch einer Auswertung dieser Knochenkonzentrationen als in vivo generierte, ernährungsabhängige Elementsigna- 
turen ist nach den vorliegenden Auswertungen statistisch nicht haltbar ${ }^{5}$ (vgl. Abbildung 5, und Tabelle 1).

Da sich bei Weingarten alle weiteren in 2.1 (S. 19ff) dargestellten Alternativhypothesen statistisch als irrelevant ergeben haben, bleibt als Phänomen nur festzuhalten, daß diagenetisch stärker veränderte Knochenproben höhere $\mathrm{Ba} / \mathrm{Ca}$ - und $\mathrm{Sr} / \mathrm{Ca}-W e r t e$ aufweisen.

Dieses eindeutige Konzentrationsmuster wurde für die beiden anderen statistisch neu ausgewerteten Populationen Wenigumstadt und Kirchheim unter Teck (vgl. Abbildung 6 und Tabelle 2) sowie für die im Probenaufreinigungsversuch untersuchte Stichprobe von Kirchheim am Ries bestätigt (vgl. Abbildung 8 und Tabelle 3). Eine mit diagenetischen Veränderungen der Knochensubstanz (schlechte histologische Erhaltung) einhergehende Veränderung der $\mathrm{Ba} / \mathrm{Ca}$ - und $\mathrm{Sr} / \mathrm{Ca}-W e r t e$ ist damit für die Skelettfunde von vier verschiedenen Bestattungsorten evident. Hieraus folgt im Umkehrschluß, daß eine unterschiedliche histologische Erhaltung der Knochenbinnenstruktur einer differentiellen chemischen diagenetischen Veränderung des Knochenminerals entspricht.

\footnotetext{
${ }^{5}$ Die daraus resultierende Kritik der früheren Interpretation (Schutkowski et al. 1999) ist unter Hinweis auf die prinzipiell unterschiedliche Herangehensweise der Dateninterpretation unbedingt zu relativieren. Mit der ansatzfreien Clusteranalyse erfolgte in der ursprünglichen Auswertung eine explorative Interpretation gemessener Daten. Die Ernährungsgruppen wurden aus den für die Individuen gemessenen Elementgehalten als Konzentrationsgruppen (induktiv) abgeleitet. Diese über die Konzentrationen definierten Gruppen wurden anschließend in Bezug zur möglichen Ursache in Form der Sozialgruppen gestellt, d.h. die Individuen der beiden Sozialgruppen wurden in die clusteranalytisch ermittelten Konzentrationsgruppen eingeordnet und dabei Entsprechungen beobachtet. Diese Vorgehensweise, Konzentrationsgruppen als Ernährungsgruppen zu interpretieren, ist daher als hypothesenbildend zu betrachten. Die in der vorliegenden Arbeit realisierte Statistik verwendete nicht die Konzentrationsgruppen als Bewertungsbasis, sondern von den Konzentrationsmessungen unabhängige externe Gruppen wurden als (deduktiv) gegebene, zu prüfende Hypothesen betrachtet. Die Hypothese der diagenetischen (histologisch definierten) Gruppen wurde den alternativen Gruppenkonstrukten (Sozialgruppen, Geschlechtergruppen) gegenübergestellt, d.h. hier wurden die Konzentrationsdaten der Individuen in die verschiedenen Gruppen eingeordnet und anschließend die daraus resultierenden Konzentrationsgruppen auf Unterschiede überprüft (vgl. 2.1, S. 19ff).
} 


\section{Der systematische Charakter der diagenetischen Veränderungen der $\mathrm{Ba} / \mathrm{Ca}$ - und $\mathrm{Sr} / \mathrm{Ca}$ - Werte}

Wurde im Ergebnisteil bewußt wertfrei nur von systematisch höheren $\mathrm{Ba} / \mathrm{Ca}$ - und $\mathrm{Sr} / \mathrm{Ca}$ Werten gesprochen, so wird an dieser Stelle literaturkonform konstatiert, daß bei Vorhandensein diagenetischer Veränderungen diese meist zur Erhöhung der in vivo generierten $\mathrm{Ba} / \mathrm{Ca}-$ und Sr/Ca-Werte führen (z.B. Tuross et al. 1989; Pate et al. 1989; Pate et al. 1991). Hierbei ist anzumerken, daß auch unsystematische Änderungen (Klepinger et al. 1986) und für Strontium sogar Konzentrationsverringerungen festgestellt wurden (Schoeninger et al. 1989). Bei letztgenannter Untersuchung erfolgte aber kein Bezug auf die Calciumkonzentrationen. Diese ist daher nur eingeschränkt aussagekräftig.

Für die vorliegende Untersuchung resultiert die Feststellung einer systematischen Erhöhung der Konzentrationsverhältnisse $(\mathrm{Ba} / \mathrm{Ca}$ und $\mathrm{Sr} / \mathrm{Ca})$ aus dem Vergleich der drei Populationen Kirchheim unter Teck, Wenigumstadt und Weingarten hinsichtlich des unterschiedlichen Grades der Divergenz zwischen den beiden Histologiegruppen (als Maß für den Grad der populationsinternen diagenetischen Divergenz). Eine Zunahme der Divergenz zwischen den Histologiegruppen (von Kirchheim unter Teck über Wenigumstadt zu Weingarten) ist Folge stärker erhöhter Werte der schlecht erhaltenen Knochen gegenüber den gut erhaltenen (besonders deutlich bei $\mathrm{Sr} / \mathrm{Ca}$, vgl. Abbildung 6).

Der angesprochene unterschiedliche Grad der populationsinternen Konzentrationsdivergenz zwischen den Histologiegruppen kann in Anbetracht der Belegungszeiträume der drei Gräberfelder innerhalb des gleichen Zeitrahmens (4. bis 8 Jh., vgl. 3.1.1) nicht als Zeitphänomen gewertet werden. Er ist daher als Folge spezifischer Bodenbedingungen der Gräberfelder einzuschätzen (vgl. Hedges et al. 1995; Nielsen-Marsh \& Hedges 2000a). "Spezifische" Bodenbedingungen bedeutet aber unter Beachtung der für alle Gräberfelder gleichgerichteten systematischen diagenetischen Konzentrationsveränderungen nur "Spezifität" hinsichtlich mehr oder minder diagenesebegünstigender Bedingungen (vgl. z.B. Gordon \& Buikstra 1981; Hedges \& Millard 1995). Trotz der unterschiedlichen geographischen Lage der drei Bestattungsorte (maximale Distanz Wenigumstadt - Weingarten ca. $300 \mathrm{~km}$ ) und damit möglicherweise variierenden Bodenkonzentrationen bleibt die Richtung der diagenetischen Konzentrationsänderungen im Knochen unbeeinflußt.

Die gräberfeldintern unterschiedliche Erhaltung der Knochenproben mit daraus resultierenden unterschiedlich veränderten $\mathrm{Ba} / \mathrm{Ca}$ - und $\mathrm{Sr} / \mathrm{Ca}$-Werten wird mit lokaler (auf das 
Gräberfeld beschränkter) Variabilität der das Knochenmineral beeinflussenden Bodenbedingungen erklärt (vgl. z.B. Hanson \& Buikstra 1987).

Die Erklärung für den systematischen Charakter der Konzentrationsänderungen erscheint laut Literatur komplexer. Es sei daran erinnert, daß verschiedene Studien ebenfalls systematische Konzentrationsveränderungen ergeben haben. Unter Hinweis auf eine Reihe von Autoren resümieren Sandford \& Weaver (2000), "...such changes may not be uniform or easy predict..." (S. 335). Dieselben Autoren betonen gleichzeitig den inzwischen verstärkten Zweifel, daß Elementeinträge in den Knochen infolge eines Konzentrationsgradienten zwischen Boden und Knochen erfolgen. Auch wenn es mit der Einschränkung auf gelöste, mithin mobile Ionen erfolgte, präferierten Pate et al. (1989) noch eine solche Angleichung zwischen Knochenkonzentration und Bodenlösung.

Drei Teilergebnisse dieser Arbeit zusammen betrachtend, kann die systematische Anreicherung des Knochenminerals mit Barium und Strontium in Relation zu Calcium vielleicht einfacher erklärt werden:

- Innerhalb einer Skelettpopulation sind die $\mathrm{Ba} / \mathrm{Ca}$ - und $\mathrm{Sr} / \mathrm{Ca}-W e r t e$ der diagenetisch

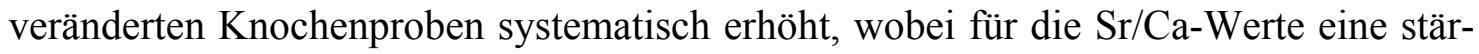
kere Zunahme zu beobachten ist (vgl. Abbildung 7 und Abbildung 9).

- Obwohl es diagenetische Erhöhungen der Barium- und Strontiumgehalte der Knochenproben gegeben hat, bleibt die theoretisch nur bei ernährungsbedingten $\mathrm{Ba} / \mathrm{Ca}$ - und $\mathrm{Sr} / \mathrm{Ca}-W e r t e n ~ z u$ erwartende Korrelation zwischen diesen Konzentrationsquotienten (vgl. 2.3, S. 27f) innerhalb der Gruppe der diagenetisch veränderten Knochenproben erhalten (vgl. Abbildung 11 und Abbildung 12). Die Konzentrationsänderungen beider Elemente müssen hiernach proportional gewesen sein.

- Innerhalb einer Knochenprobe wird ein von Periost zum Markraum verlaufender Konzentrationsgradient für $\mathrm{Ba} / \mathrm{Ca}$ und $\mathrm{Sr} / \mathrm{Ca}$ beobachtet. Veränderte Mineralareale zeigen aber unabhängig davon erhöhte und miteinander korrelierende Werte beider Quotienten (vgl. Abbildung 27 und Abbildung 28).

Diese Beobachtungen können mit einer (Rand-)bemerkung von Pate \& Brown (1985), die sich aus der Theorie der Ernährungsrekonstruktion herleitet, in Zusammenhang gebracht werden: "Once bone enters the geochemical environment it is no longer subject to the biological discrimination against strontium." (S. 487; wobei nicht ausgeschlossen werden soll, daß diese "simple" Beobachtung nicht auch von anderen gemacht wurde). Man beachte, 
daß hier der Erstautor derselbe ist, der einige Jahre später für den Konzentrationsausgleich zwischen Boden und Knochen ungeachtet der Konzentrationsquotienten von $\mathrm{Sr} / \mathrm{Ca}$ und Ba/Ca "plädiert" (Pate et al. 1989, s.o.), obwohl in seiner früheren Aussage ein vielleicht einfacherer Erklärungsansatz für systematische Konzentrationserhöhungen bereits implizit enthalten ist. Er erinnert daran, daß biologische Systeme gegen Strontium (und wenn auch nicht genannt, analog gegen Barium) zugunsten von Calcium diskriminieren (vgl. 1.1, S. 2f).

Der Mensch ist in das Nahrungsnetz eingebunden. Folglich ist sein Knochenmineral gegenüber dem Startpunkt des Diskriminierungsprozesses, dem Boden seines Habitats, an Barium und Strontium in Relation zu Calcium abgereichert. Dies bedeutet wiederum, daß der Knochen nach dem Tod in einen Boden gelangt, dessen $\mathrm{Ba} / \mathrm{Ca}-\mathrm{bzw}$. Sr/Ca-Relationen (einzig aus der Theorie der Ernährungsrekonstruktion abgeleitet) höher sein müssen als seine eigenen. Ohne die Protektion der biologischen Diskriminierung wirkt nur noch die um ein vielfaches geringere mineralchemische Diskriminierung gegen Barium und Strontium (vgl. Stark 1968). Jeglicher mineralchemischer diagenetischer Prozeß, ob nur Ionensubstitution oder Re- bzw. Neukristallisationen, müßte hiernach prinzipiell zu höheren $\mathrm{Ba} / \mathrm{Ca}$ - und $\mathrm{Sr} / \mathrm{Ca}-W e r t e n$ im Knochen gegenüber den in vivo generierten Verhältnissen führen, ähnliche Mobilität aller beteiligten Elemente entsprechend ihrer ähnlichen Chemie vorausgesetzt. Die Situation für das Knochenmineral ist prinzipiell die gleiche wie zu Lebzeiten. In Abhängigkeit von der angebotenen Relation der Elemente zueinander erfolgt der Einbau in das Mineralkristall, nur daß diese Relation zu Lebzeiten im Vergleich zur Situation im Boden zu Gunsten von Calcium verschoben ist. Die Absolutkonzentrationen von Barium und Strontium im Boden können allein nicht für eine Erklärung der Konzentrationserhöhung im Knochen herangezogen werden.

Aus der ähnlichen Chemie von Barium und Strontium folgt ebenfalls, daß beide Elemente proportional in das diagenetisch veränderte Mineral eingebunden werden. Die primär ernährungsbedingte Korrelation zwischen den Konzentrationsverhältnissen beider Elemente innerhalb einer Skelettpopulation würde zwangsweise auch im Verlauf der Diagenese erhalten bleiben. Die stärkere Konzentrationszunahme für Strontium gegenüber Barium erklärt sich aus dessen kleineren Ionenradius, welcher auch mineralchemisch eine geringere Diskriminierung als gegen Barium zur Folge hat.

Hiermit ist auch der gleichlaufende Konzentrationsgradient innerhalb der mikroanalytisch untersuchten Knochenprobe zu erklären. Oberflächennahe (und damit bodennahe) Mine- 
ralareale gleichen ihre Elementrelationen diffusionsbedingt denen im Boden schneller an als bodenferne. Da dieser Gradient auch unabhängig von erkennbaren Mineralumbildungen als eigene Komponente zu beobachten ist, resultiert er wahrscheinlich hauptsächlich aus Ionensubstitutionen. Die beobachteten lokalen Maxima der $\mathrm{Ba} / \mathrm{Ca}-$ und $\mathrm{Sr} / \mathrm{Ca}-\mathrm{Werte}$ innerhalb des Kompaktaquerschnittes (vgl. Abbildung 27) sind mit umfangreicheren mineralischen Umkristallisationen in den als verändert angesprochenen Kompaktaarealen verbunden. Diese führen nach den vorliegenden Ergebnissen zu einer forcierten Änderung der Konzentrationsrelationen des Knochens.

Inwieweit Verteilungskoeffizienten zwischen Bodenlösung und Knochenmineral für eine Quantifizierung dieser Konzentrationsveränderungen verwendet werden könnten, müßte in zukünftigen Untersuchungen geklärt werden. Deren Bestimmung ist prinzipiell problematisch und dürfte infolge der mehrphasigen Komposition des Knochenminerals besonders komplex sein (allgemein zu Verteilungskoeffizienten zwischen Mineral und Lösung für Strontium vgl. z.B. Banner 1995).

An dieser Stelle kann zunächst zusammenfassend festgestellt werden, daß die $\mathrm{Ba} / \mathrm{Ca}$ - und $\mathrm{Sr} / \mathrm{Ca}-W e r t e$ sich während der Diagenese erhöhen und dies eventuell aus der primären biologischen Diskriminierung gegen Barium und Strontium zu erklären ist. In deren Folge hat der Boden gegenüber dem Knochen erhöhte $\mathrm{Ba} / \mathrm{Ca}$ - und $\mathrm{Sr} / \mathrm{Ca}-W e r t e$. Ein "Konzentrationsausgleich" zwischen Boden und Knochen würde unter dieser Annahme auf der Grundlage dieser unterschiedlichen Konzentrationsverhältnisse erfolgen und nicht in Abhängigkeit vom Konzentrationsgradienten der Absolutkonzentrationen.

Ein Aspekt, der an dieser Stelle schon abschließend behandelt werden kann, ist die Beobachtung, daß die nahrungsbedingte Korrelation zwischen $\mathrm{Ba} / \mathrm{Ca}-$ und $\mathrm{Sr} / \mathrm{Ca}-W e r t e n$ unabhängig von der diagenetischen Veränderung des Knochenminerals erhalten bleibt. Dies wird mit der chemisch bedingten proportionalen Barium- und Strontiuminkorporation im Verlauf der Diagenese begründet. Folge dieses Ergebnisses einschließlich des Erklärungsansatzes ist die "Disqualifikation" des Strontium-Barium-Zusammenhanges als Kontrollkriterium zur Erfassung diagenetischer Veränderungen. Die von Burton \& Price (1999) vorgeschlagene Korrelationsprüfung für die $\mathrm{Ba} / \mathrm{Ca}$ - und $\mathrm{Sr} / \mathrm{Ca}-W e r t e$ ermöglicht (für die vier untersuchten Gräberfelder) keine Differenzierung zwischen unterschiedlich diagenetisch veränderten Knochenproben. Diagenetische Änderungen der $\mathrm{Ba} / \mathrm{Ca}$ - und $\mathrm{Sr} / \mathrm{Ca}-\mathrm{Werte}$ im Mineral bodengelagerten Skelettmaterials sind nicht unsystematisch, wie von diesen Autoren angenommen. 
Für die Spurenelementuntersuchungen an bodengelagertem Skelettmaterial bleibt die Frage zu klären, warum diese Prozesse der Konzentrationserhöhung von Barium und Strontium durch eine Probenaufreinigung nicht reversibel sind. Die infolge Neu- bzw. Rekristallisation veränderten Mineralareale innerhalb einer Knochenprobe sollten laut Aufreinigungstheorie leichter löslich sein, als "natives" Knochenmineral.

\section{Probenaufreinigung und diageneseanzeigende Kontrollkriterien - Möglichkeiten einer erweiterten Charakterisierung der Knochendiagenese}

Die diagenetisch systematisch erhöhten $\mathrm{Ba} / \mathrm{Ca}$ - und $\mathrm{Sr} / \mathrm{Ca}$-Werte wurden in vier Gräberfeldern nach der Probenaufreinigung festgestellt. Die Daten von Weingarten, Kirchheim unter Teck und Wenigumstadt resultierten alle aus Analysen von säurebehandelten Knochenproben (vgl. 2.2). Die Proben von Kirchheim am Ries zeigten nach den verschiedenen Methoden der Probenaufreinigung nur marginale Änderungen der Elementkonzentrationen und die schlecht erhaltenen Knochenproben waren unabhängig von der Aufreinigung weiterhin durch erhöhte $\mathrm{Ba} / \mathrm{Ca}$ - und $\mathrm{Sr} / \mathrm{Ca}-W e r t e$ gekennzeichnet.

Die Untersuchung der Stichprobe von Kirchheim am Ries ermöglicht eine Bewertung der Aufreinigungsvarianten, wobei sich im Folgenden auf die Variante der Oberflächenbehandlung (ohne Ätzen) und das Ätzen (ohne Oberflächenbehandlung) beschränkt wird. Für die Beurteilung der letzteren können zusätzlich die Daten der anderen drei Skelettpopulationen verwendet werden.

Die beobachtete Ineffizienz einer ausschließlichen mechanischen Oberflächenbehandlung der Proben hinsichtlich einer Veränderung (Verringerung) der $\mathrm{Ba} / \mathrm{Ca}-$ und $\mathrm{Sr} / \mathrm{Ca}$ Werte ist relativ einfach zu erklären (vgl. Abbildung $10 \mathrm{c}+\mathrm{d}$ ). Unabhängig von einem radialen Gradienten (Abnahme von Periost nach innen) beider Konzentrationsquotienten wurden bei der mikroanalytischen Untersuchung in oberflächenfernen Mineralarealen der Knochenkompakta lokale Anreicherungen von Barium und Strontium infolge mineralischer Umbildungen beobachtet (s.o., vgl. Abbildung 27). Diese bleiben auch nach Entfernen der Oberfläche erhalten. Dieser Prozeß einer im Kompaktainneren verstärkten mineralischen Umbildung ist nach den Auswertungen der Histologien aller vier Skelettserien typisch für die diagenetische Veränderung der Knochenbinnenstruktur (vgl. 2.1, S.12ff). Folglich wird der in der Einzelprobe beobachtete Effekt der angereicherten Areale in diagenetisch veränderten Proben allgemein vorhanden sein. 
Nur für Aluminium zeigte sich bei der ausschließlichen Oberflächenbehandlung ein deutlicher Effekt (vgl. Abbildung 17b). Dieses Element scheint, durch weitere chemische Wechselwirkungen weniger beeinflußt, hauptsächlich diffusionsbedingt in das Knocheninnere zu gelangen (vgl. z.B. Quattropani et al. 1999). Letztlich bleibt aber auch für dieses Element festzustellen, daß es weit in die Kompakta eingedrungen sein muß, da es durch die Oberflächenbehandlung nicht vollständig aus den Proben entfernt werden konnte. Natives (vollständig aufgereinigtes) Knochenmineral müßte durch Aluminiumkonzentrationen unterhalb der Nachweisgrenze der in dieser Untersuchung verwendeten Analysemethoden gekennzeichnet sein (vgl. 2.3, S. 31). Man beachte, daß auch gut erhaltene Knochenproben Aluminium in bestimmbaren Konzentrationen vor und nach der Probenaufreinigung enthielten. Dies bedeutet für die Kontrollfunktion des Elementes Aluminium, daß diagenetisch unterschiedlich veränderte Knochenproben zwar im Mittel durch unterschiedlich hohe Aluminiumkonzentrationen gekennzeichnet sind, aber eine eindeutige Trennung zwischen beiden Gruppen mit diesem Kontrollkriterium nicht möglich ist.

Die fehlende Wirkung der chemischen Probenaufreinigung durch Ätzen auf die $\mathrm{Ba} / \mathrm{Ca}$ und $\mathrm{Sr} / \mathrm{Ca}-W e r t e(v g l$. Abbildung $10 \mathrm{a}+\mathrm{b}$ ) ist im allgemeinen Kontext der Spurenelementuntersuchungen an bodengelagertem Skelettmaterial ausführlicher zu bewerten. Diese Aufreinigungsvariante entspricht dem zwar nicht unumstrittenen (vgl. aktuellere Kritik von Budd et al. 2000 und Nielsen-Marsh \& Hedges 2000b), doch wissenschaftlich akzeptierten Prinzip (vgl. Sandford \& Weaver 2000). Eine mangelnde Effizienz dieses Aufreinigungsprinzips birgt grundsätzliche Probleme für zukünftige spurenelementgestützte Untersuchungen zur Ernährungsrekonstruktion. Die Erklärung für diese Ineffizienz kann nur ansatzweise unter Einbeziehung der Ergebnisse der diagenesecharakterisierenden Kontrollkriterien sowie der mikroanalytischen Untersuchungen erfolgen.

Das in den früheren Untersuchungen (zu Kirchheim unter Teck, Wenigumstadt und Weingarten) ausgewertete Kontrollkriterium war das Ca/P-Verhältnis der aufgereinigten Knochenproben (vgl. Schutkowski 1995; Schutkowski et al. 1999). Dieses Kriterium zeigt mit Ausnahme von Weingarten für alle aktuell untersuchten bzw. überprüften Serien keinen Unterschied zwischen gut und schlecht erhaltenen Proben. Außerdem weichen die Ca/PWerte der bodengelagerten Knochen nur unbedeutend von rezenten Referenzwerten ab. Hervorzuheben ist hierbei nochmals, daß diese Auswertungen für Kirchheim am Ries an den unbehandelten Knochenproben erfolgten (vgl. Abbildung 13). 
Dies bedeutet, daß diagenetische Veränderungen der Knochenbinnenstruktur (schlechte Histologie) bei den untersuchten Skelettserien nicht mit einer Veränderung des $\mathrm{Ca} / \mathrm{P}$ Wertes des Knochenminerals einhergingen. Diese Beobachtung wird zusätzlich von den mikroanalytischen Ergebnissen bestätigt. Das Ca/P-Verhältnis bleibt innerhalb eines Kompaktaquerschnittes unabhängig vom Mineralerhaltungszustand gleich (vgl. Abbildung 25). Die Unterschiede zwischen den Mineralarealen ("nativ"/verändert) waren auf die Absolutkonzentrationen beschränkt (vgl. hierzu auch Abbildung 22b und Abbildung 23b). Entsprechende Ergebnisse sind auch aus der Literatur als Hypermineralisation bekannt (Hedges et al. 1995; Pfeiffer \& Varney 2000). Inwieweit bei einem gleichbleibenden Konzentrationsverhältnis und variablen Absolutkonzentrationen von Calcium und Phosphor sich die Komposition aus den verschiedenen Calcium-Phosphor-Verbindungen geändert hat, muß in der vorliegenden Untersuchung offen bleiben. Hierfür sind kristallographische Analysen erforderlich (zu den Möglichkeiten verschiedener Techniken vgl. z.B. Peters et al. 2000; Penel et al. 1998; Bigi et al. 1997).

Entscheidende Schlußfolgerung für das Ca/P-Verhältnis bleibt, daß es gegenüber diagenetischen Veränderungen des Knochenminerals indifferent ist. Dieses Kriterium ist wie der Ba-Sr-Zusammenhang (für die vier untersuchten Gräberfelder) ungeeignet zur Unterscheidung zwischen verschiedenen Graden diagenetischer Veränderungen und wird damit seiner Kontrollfunktion nicht gerecht.

An dieser Stelle ist die Frage zu stellen, ob diese "stabile" Ca/P-Charakteristik eine hinreichende Erklärung dafür ist, daß diagenetische Veränderungen scheinbar nicht zu einer hinreichend erhöhten Löslichkeit des betroffenen Knochenminerals führen, wie es für eine effektive säureinduzierte Aufreinigung Voraussetzung wäre (vgl. 1.2, S. 7).

Histologisch schlecht erhaltene Knochenproben zeigen bei allen untersuchten Skelettserien im Vergleich zu gut erhaltenen Proben einen erhöhten Masseverlust bei der chemischen Probenaufreinigung durch Ätzen (vgl. Abbildung 18 und Tabelle 8). Dies muß aber nach Auswertung der SEM-Bilder nicht Folge größerer Anteile leichter löslichen Minerals sein, denn diagenetisch veränderte Mineralareale sind durch vermehrte Bereiche mineralfreier "Poren" gekennzeichnet (vgl. Abbildung 22a und Abbildung 23a). Hier muß die Möglichkeit in Betracht gezogen werden, daß solche Strukturen einen erleichterten Zugang des Lösungsmediums in das Knocheninnere ermöglichen und daraus ein effektiverer Lösungsprozeß resultiert (zur Porosität vgl. Nielsen-Marsh \& Hedges 1999; Nielsen-Marsh \& Hedges 2000a). 
Für den Masseverlust bei der chemischen Probenaufreinigung ist unabhängig von den möglichen Ursachen festzustellen, daß er zwischen Proben mit unterschiedlichem Erhaltungszustand differenziert.

Erhöhte Löslichkeit diagenetisch veränderten Knochenminerals wird insbesondere mit einem erhöhten Carbonatgehalt in Verbindung gebracht. Dies wird mit der durch Carbonat verringerten Kristallinität begründet. Hierauf beruft sich letztlich auch Sillen (1986) bei der Etablierung seiner Aufreinigungsmethode, von welcher sich die verschiedenen verwendeten Modifikationen, einschließlich der in der vorliegenden Arbeit, ableiten. Eine diagenetische Carbonatinkorporation in das Knochenmineral wird hierfür aber nach Chickerur et al. (1980) als gekoppelte Substitution von Natrium und Carbonat für Calcium und Phosphat (vgl. 2.3, S. 30) angenommen. Dies sollte für diagenetisch veränderte Knochenproben im Falle erhöhter Carbonatgehalte auch erhöhte Natriumgehalte bedeuten. Die Ergebnisse der vorliegenden Arbeit besagen aber genau das Gegenteil. Muß für die Stichprobe von Kirchheim am Ries noch die Möglichkeit in Betracht gezogen werden, daß die niedrigeren $\mathrm{Na} / \mathrm{Ca}-W e r t e$ der schlecht erhaltenen Proben aus einem möglichen Aufreinigungseffekt resultieren (es wurden nur aufgereinigte Proben analysiert, vgl. Abbildung 29), ist dies bei den mikroanalytischen Ergebnissen ausgeschlossen. Sowohl die Verteilungsbilder als auch die Einzelpunktanalysen zeigen, daß veränderte Mineralareale im Vergleich zu "nativen" durch geringere Natriumgehalte bzw. Na/Ca-Werte gekennzeichnet sind (vgl. Abbildung 22d und Abbildung 23d sowie Abbildung 26). Diese Beobachtung wird wiederum in der Literatur bestätigt (z.B. Reiche et al. 1999). Entweder erfolgt die Carbonatinkorporation in veränderte Mineralareale durch einen anderen chemischen Mechanismus, oder man könnte annehmen, daß im vorliegenden Fall verändertes Knochenmineral nicht mit Carbonat angereichert und daher auch nicht durch diesen Mechanismus leichter löslich ist. Damit wäre eine weitere Erklärungsmöglichkeit für das Versagen der chemischen Probenaufreinigung gegeben.

Als Nebenaspekt ist festzustellen, daß Natrium als Kontrollkriterium zwischen unterschiedlich veränderten Knochenproben (bzw. innerhalb einer Knochenprobe zwischen "nativen" und veränderten Mineralarealen) sehr gut differenziert. Hierbei ist zu beachten, daß auch gut erhaltene Knochenproben deutlich niedrigere Natriumgehalte aufweisen als rezentes Vergleichsmaterial (vgl. Abbildung 29).

Magnesium wird zusammen mit Carbonat im Rahmen eines "synergetischen Effektes" in Richtung einer erhöhten Löslichkeit genannt (LeGeros et al. 1995). Die Ergebnisse der 
vorliegenden Arbeit erlauben allerdings keine Interpretation in diesem Sinne. Magnesium ist wie Natrium in gut erhaltenen Knochen bzw. innerhalb eines Knochens in "nativen" Mineralarealen in höheren Konzentrationen ( $\mathrm{Mg} / \mathrm{Ca}-W e r t e n)$ enthalten (vgl. Abbildung 14 sowie Abbildung 22c und Abbildung 23c). Da der Vergleich mit rezenten Konzentrationen zeigt, daß bodengelagertes Knochenmaterial generell niedrigere Konzentrationen aufweist, müssen die geringeren Gehalte in schlecht erhaltenen Knochenproben bzw. veränderten Mineralarealen als ein diagenetisch bedingtes Herauslösen des Magnesiums aus der Knochensubstanz verstanden werden.

Durch den unterschiedlichen Grad dieses Herauslösens differenziert Magnesium als Kontrollkriterium sehr effektiv zwischen unterschiedlichen Erhaltungszuständen. Dies allerdings mit der gleichen Einschränkung wie für Natrium (s.o.), da auch die gut erhaltenen Knochenproben schon verringerte Gehalte aufweisen.

Zur Frage der Löslichkeit diagenetisch veränderten Knochenminerals kann diese Beobachtung nur soviel beitragen, daß dieser Lösungsprozeß im Zuge der diagenetischen Veränderungen bei histologisch schlecht erhaltenen gegenüber gut erhaltenen Knochenproben beschleunigt verlaufen ist. Dieser Aspekt erlangt insofern Bedeutung, als daß dieses beschleunigte Herauslösen von Magnesium sich bei den schlecht erhaltenen Proben während der chemischen Probenaufreinigung fortsetzt. Diese Proben verlieren unter Säureeinwirkung systematisch überproportional mehr Magnesium als Calcium (die $\mathrm{Mg} / \mathrm{Ca}-W e r t e$ verringern sich). Hingegen verhalten sich die $\mathrm{Mg} / \mathrm{Ca}-W e r t e$ der gut erhaltenen Proben gegenüber dem Ätzen indifferent. Die Werte erhöhen sich eher noch, was bedeuten würde, daß diese Proben überproportional mehr Calcium verlieren (vgl. Abbildung 15a+c).

Als Ursache für diesen Effekt kann natürlich der erleichterte Lösungsprozess bei schlecht erhaltenen Proben infolge der erhöhten Porosität (vgl. S. 137) angeführt werden. Es wird jedoch unter Einbeziehung der beiden bisher in die Diskussion noch nicht integrierten Variablen zur Diagenesecharakterisierung (Schwefelgehalt und Masseverlust beim Veraschen) eine Alternativargumentation vorgeschlagen:

Bedeutet ein erhöhter Magnesiumgehalt eine erhöhte Löslichkeit (s.o.) und ist Magnesium in vivo in einer relativ leicht löslichen Mineralkomponente enthalten (vgl. 2.3, S.30), könnte ein trotzdem verringerter Lösungseffekt bei gut erhaltenen Proben eventuell als Folge einer Protektion gut erhaltener Mineralareale interpretiert werden. Aus den Einzelpunktmessungen der EPMA resultiert für Schwefel die gleiche Charakteristik wie für Magnesi- 
um und Natrium. Meßpunkte in "nativen" Mineralarealen sind gegenüber denen in veränderten durch deutlich erhöhte Schwefelgehalte (S/Ca-Werte) gekennzeichnet (vgl. Abbildung 26). Schwefel als Sulfat wird aber als mineralische Komponente nicht genannt (vgl. Driessens 1980). Für sulfathaltige Moleküle der organischen Matrix wird jedoch diskutiert, daß diese gerade über das Sulfat an (in) das Kristallgitter assoziiert (integriert) sind (zu schwefelhaltigen organischen Verbindungen im Knochen vgl. Bilezikian et al. 1996, S. 119, zur spezifischen Bindung vgl. Embery \& Rolla 1980). Hiernach könnte ein erhöhter Schwefelgehalt als Marker für erhöhten Organikgehalt in den "nativen" Mineralregionen verstanden werden. Die geringeren Gehalte in den veränderten Regionen wären entsprechend ein Indiz für einen Verlust organischer Bestandteile. Dies wiederum wäre in Übereinstimmung mit den Beobachtungen, daß die Zerstörung der Knochenbinnenstruktur durch mikrobielle Organikzerstörung initiiert wird (vgl. 2.3, S. 32f; zur mikrobiellen Aktivität im Knochen allgemein vgl. Child 1995).

Die Masseverluste beim Veraschen der Knochenproben unterstützen diese Hypothese. Bei allen untersuchten Skelettserien (einschließlich Kirchheim am Ries mit dem charakteristischen Magnesiumverlust durch das Ätzen) zeigen für gut erhaltene Knochenproben einen Masseverlust beim Veraschen, der rezentem Material entspricht, für schlecht erhaltene Proben deutlich geringere Masseverluste (vgl. Abbildung 19).

Insbesondere bleibt für den Masseverlust beim Veraschen festzuhalten, daß dieser im Vergleich zu allen anderen im Rahmen der Untersuchung getesteten Kontrollkriterien zur Differenzierung zwischen unterschiedlichen Graden der diagenetischen Veränderung die zuverlässigste Trennung zwischen den beiden histologisch definierten Gruppen ermöglicht. Hiernach besteht eine deutliche Abhängigkeit zwischen histologischem Erhaltungszustand und dem im Knochen noch vorhandenen Organikgehalt. Diese Beobachtung steht im Widerspruch zu den Ergebnissen von Hedges et al. (1995), die anhand ihrer Daten der histologischen Kategorisierung von Knochenproben ein solches Abschätzungsvermögen für den Organikanteil absprechen.

\section{Schlußfolgerungen für die Interpretationsmöglichkeiten von Spurenelementkonzentrationen in bodengelagertem Skelettmaterial}

Diagenetische Veränderungen der Knochensubstanz werden inzwischen als Problem für die Interpretation von Spurenelementdaten akzeptiert. Auswertungen und Interpretationen werden aber weiterhin fortgesetzt. 
Die allgemeine Einschätzung ist, daß die Richtung diagenetischer Konzentrationsveränderungen nicht vorhersagbar ist. Hiervon hebt sich die vorliegende Untersuchung ab. Die Beobachtung von erhöhten Konzentrationen in diagenetisch stärker veränderten Knochenproben bei vier untersuchten Gräberfeldern führte zu dem vorsichtigen Versuch, dies als systematisch zu interpretieren und eine naheliegende Erklärung auf der Grundlage der Prinzipien der Ernährungsrekonstruktion abzuleiten.

Die Verwendung und Überprüfung der seit nun mehr als 15 Jahren hinsichtlich ihrer prinzipiellen Tauglichkeit wiederholt angezweifelten Problemlösungsstrategie in Form einer chemischen Probenaufreinigung hatte ein ernüchterndes Ergebnis zur Folge. Die diagenetischen Konzentrationsveränderungen der nahrungsanzeigenden Elemente Barium und Strontium sind unter Verwendung dieser Methode bei allen vier untersuchten Skelettserien nicht reversibel.

Mit dem Ziel einer differenzierteren Charakterisierung diagenetischer Prozesse stützen sich Erklärungsversuche für diese Beobachtung auf die Auswertung von knochensubstanzspezifischen Parametern. Dies entspricht der momentan empfohlenen und praktizierten Vorgehensweise zum Diageneseproblem bei Spurenelementuntersuchungen an bodengelagertem Skelettmaterial (vgl. Sandford \& Weaver 2000).

Für ein langjährig präferiertes und entsprechend in vielen Untersuchungen genutztes Kontrollkriterium $(\mathrm{Ca} / \mathrm{P}-$ Verhältnis) ergeben die Auswertungen, daß es ungeeignet ist, diagenetische Mineralveränderungen anzuzeigen (vgl. Radosevich 1993; Hedges et al. 1995 Pfeiffer \& Varney 2000). Eine schon vor 10 Jahren vorgeschlagene (Burton \& Price 1990a) und noch vor zwei Jahren (Burton et al. 1999) als praktikable Alternative betrachtete Kontrollmöglichkeit des Barium-Strontium-Zusammenhanges erweist sich ebenfalls als unzureichend. Die weiteren genutzten Parameter (Mg-, Na- Al-Konzentrationen sowie die beiden spezifischen Masseverluste während der Probenvorbereitung) ermöglichten in einem begrenzten Rahmen eine erweiterte Phänomenologie der Diagenese. Schlußfolgerungen hinsichtlich des bisherigen Erklärungsansatzes der differentiellen Löslichkeit von diagenem und biogenem Mineral, der sowohl diagenetische Veränderungen charakterisieren soll als auch die Grundlage für das Aufreinigungsprinzip bietet, wurden entsprechend nur unter Vorbehalt angeführt.

Eine weitere Nutzung dieser Kontrollkriterien wird dadurch eingeschränkt, daß auch diejenigen Variablen, die zwischen unterschiedlichen Graden der diagenetischen Veränderung 
des Knochens differenzieren, keine Quantifizierung der Konzentrationsänderungen der ernährungsanzeigenden Elemente Barium und Strontium ermöglichen. Dies wäre nur möglich, wenn zwischen den hier verwendeten bzw. weiteren diagenesecharakterisierenden Variablen innerhalb eines theoretischen Rahmens quantifizierte Zusammenhänge vorhersagbar wären. Eines solchen Systems ermangelt es aber bisher und es erscheint nach den bisherigen Versuchen auch nicht sehr wahrscheinlich (vgl. Hedges et al. 1995).

Das Problem der nicht quantifizierbaren diagenetischen Konzentrationsveränderungen schließt insbesondere ein, daß es keine Aussagemöglichkeit hinsichtlich einer gesicherten in vivo Bedingtheit der Spurenelementkonzentrationen von bodengelagertem Knochenmaterial gibt. Die dokumentierten und erläuterten Resultate für die Magnesium- bzw. Natriumgehalte in den als histologisch gut eingeordneten Knochenproben liegen deutlich unterhalb der rezenten Referenzwerte. Also auch ein histologisch gut erhaltener Knochen weist bereits diagenetische Veränderungen seiner Mineralzusammensetzung auf. Ohne quantifizierten Bezug, der besagt, daß trotz dieser Konzentrationsverringerungen für Natrium und Magnesium Konzentrationsänderungen für Barium und Strontium entweder auszuschließen oder aber rechnerisch $\mathrm{zu}$ korrigieren sind, ist eine Interpretation dieser Konzentrationen als ernährungsanzeigend als fraglich zu bewerten.

Der Mangel eines theoretischen Rahmens für diagenetische Veränderungen des Knochenminerals ist nicht zuletzt Folge des begrenzten Wissens über die biochemischen Mechanismen des Knochenminerals in vivo (vgl. einleitende Zitate der Diskussion, S. 129).

Der letzte in dieser Arbeit untersuchte Aspekt verdeutlicht ein solches Problem, das aus der unzureichenden Kenntnis knochenphysiologischer Zusammenhänge resultiert. Eventuell ist das erörterte Problem der intraossären Variabilität sogar noch von größerer Bedeutung für die Auswertung von Spurenelementdaten bodengelagerten Skelettmaterials als die Diagenese.

\section{Die intraossäre Konzentrationsvariabilität: Elementverteilung im Knochenmineral - undifferenziert oder knochenphysiologisch systematisch?}

Voraussetzung für die Interpretation von Barium und Strontiumkonzentrationen als Ernährungsanzeiger war die Annahme, daß diese ohne physiologische Relevanz bzw. Präferenz ausschließlich nahrungsabhängig in das Knochenmineral integriert werden. 
Die beobachtete intraossäre Variabilität der $\mathrm{Sr} / \mathrm{Ca}-$ Werte in einem rezenten Femur läßt infolge ihres systematischen Charakters bezüglich der Erfüllung dieser Prämisse Zweifel aufkommen (vgl. Abbildung 30). Im anterioren Bereich des Femurs wird ein Maximum der $\mathrm{Sr} / \mathrm{Ca}-W e r t e$ im Vergleich zu den anderen Kompaktabereichen über die gesamte Länge des untersuchten Diaphysenabschnittes beobachtet. Es ist zu fragen, ob es eine knochenphysiologische Erklärung für dieses Sr/Ca-Maximum gibt.

Unabhängig von der variablen Kompktastärke innerhalb eines Femurs konnten Amtmann \& Schmitt (bereits 1968) zeigen, daß im anterioren Bereich des Femurs die Materialdichte im Vergleich zu den medialen und lateralen Arealen deutlich geringer ist. Dies kann einerseits aus einer erhöhten Porosität (in vivo) der Kompakta resultieren, andererseits kann eine geringere Materialdichte zusätzlich durch eine geringere Mineraldichte bedingt sein. Letztere wäre kristallchemisch aus einem erhöhten $\mathrm{Sr} / \mathrm{Ca}-$ Verhältnis erklärbar. Eine vermehrte Anzahl von Strontiumionen bedeutet eine verstärkte Störung des Kristallgitters bzw. eine verringerte Kristallinität ("Ordnung") des Knochenminerals, was einer geringeren Dichte des Minerals entspricht (vgl. Grynpas 1993).

Das Vorhandensein eines solchen Zusammenhanges zwischen Mineraldichte und Position des $\mathrm{Sr} / \mathrm{Ca}$-Maximums würde bedeuten, daß sich in Abhängigkeit von der Lage der Region mit der geringsten Mineraldichte die Position des Sr/Ca-Maximums ebenfalls verschieben würde. Wenn unabhängig vom Größenwachstum im Zuge funktionsmorphologischer Anpassungen an variable Belastungen einschließlich Altersanpassungen an eine veränderte Stellung des Gesamtskeletts eine Verschiebung dieser Region angenommen wird, bedeutet dies neben einer individuellen auch eine alters bzw. belastungsabhängige Lage des $\mathrm{Sr} / \mathrm{Ca}-$ Maximums ${ }^{6}$.

Aus einer standardisierten Probenentnahme für eine Elementanalyse, die dann individuell variabel neben dem Sr/Ca-Maximum läge, würden infolge des deutlichen Konzentrationsgefälles von dem anterioren Maximum nach lateral bzw. medial (vgl. Abbildung 30) für die beprobten Individuen unterschiedliche Sr/Ca-Wert als Folge des Verteilungsmuster (artifiziell) resultieren. Eine möglicherweise in der Bevölkerung vorhandene ernährungs-

\footnotetext{
${ }^{6}$ Die Beobachtung, daß frisch gebildete Apatitkristalle (in vitro) zu einer verstärkten Inkorporation von Strontiumionen neigen (vgl. Cazalbou et al. 2000), wäre ein Indiz dafür, daß der Bereich mit dem Sr/CaMaximum eine relativ aktive Modellierungszone des Knochens ist. Die von lateral nach anterior zur Linea aspera (als ebenfalls aktive Knochenumbauzone) teilweise wieder zunehmenden $\mathrm{Sr} / \mathrm{Ca}-\mathrm{Werte}$ wären dann ebenfalls dahingehend zu interpretieren (vgl. Abbildung 30).
} 
bedingte Konzentrationsvariabilität würde hierdurch funktionsphysiologisch verfälscht werden.

Diese Untersuchung an einem Femur bedarf der unbedingten Prüfung. Es gilt sowohl die systematische Form des Konzentrationsmusters als auch mögliche individuelle Verschiebungen des Maximums zu überprüfen.

Das Resultat der Arbeit ist aber auch als Einzelergebnis relevant. Es zeigt, daß eine sehr große intraossäre Variabilität möglich ist. Ein Aspekt, der in dieser Deutlichkeit bisher keine Berücksichtigung in der Literatur gefunden hat.

\section{Schlußbemerkungen}

Spurenelementkonzentrationen werden im Rahmen der Ernährungsrekonstruktion inzwischen weniger intensiv ausgewertet. Das Forschungsinteresse hat sich auf die Verwendung von Isotopen der organischen Matrix verlagert (Kohlenstoff und Stickstoff, vgl. Sealy 2001). Aus der mineralischen Matrix wird jedoch das Strontium-Isotopenverhältnis im Zusammenhang mit Provenienzstudien analysiert. Für diesen Forschungsbereich sind die Probleme einer diagenetischen Veränderung des Knochenminerals, wie sie in dieser Arbeit untersucht wurden, wiederum von Bedeutung. Eine Inkorporation von Strontium aus dem Boden bedeutet unabhängig von einer Konzentrationserhöhung auch eine Änderung des Isotopenverhältnisses in Richtung der Bodensignatur. Die Diagenesediskussion wird in diesen Bereich entsprechend weitergeführt (vgl. Grupe et al. 1997 u. 1999 vs. Horn \& Müller-Sohnius 1999 sowie Budd et al. 2000).

Bezüglich der Knochensubstanz als chemische Informationsquelle ist nach den Ergebnissen abschließend festzustellen, daß die Diagenese der chemischen Information des Knochenminerals bis jetzt nur unzureichend charakterisierbar und insbesondere nicht quantifizierbar ist. Das Problem einer möglichen knochenphysiologisch bedingten Variabilität nahrungsanzeigender Spurenelemente zeigt, daß auch der Aspekt der Informationsgenese in vivo intensiver weiterer Forschungen bedarf.

Die Komplexität des Knochenminerals und seiner Wechselwirkungen sollte das wiederholte Verkennen von diagenetischen und knochenphysiologische Problemen bei der Auswertung von Spurenelementkonzentrationen entschuldigen. Man beachte in diesem 
Zusammenhang den Namen der knochenmineralischen Hauptkomponente, Apatit (von $\alpha \pi \alpha \tau \alpha \omega$, sich täuschen), den dieses Mineral infolge seiner primär schwierigen Charakterisierung bekommen haben soll (vgl. Elliot 1994, S. 5). 


\section{$6 \quad$ Zusammenfassung}

Im Rahmen der Ernährungsrekonstruktion von historischen Bevölkerungen sind Auswertungen von Spurenelementgehalten bodengelagerten Knochenmaterials etabliert. Voraussetzung für die Interpretation nahrungsanzeigender Elementkonzentrationen ist, daß die gemessenen Elementgehalte in vivo bedingt sind. Konzentrationsveränderungen infolge diagenetischer Prozesse unter der Liegezeit können jedoch nicht ausgeschlossen werden. Die Diskussion zu den Problemen der Charakterisierung und Quantifizierung diagenetischer Veränderungen sowie zu Möglichkeiten ihrer Reversibilität vor der Elementanalyse wird in der Literatur seit längerem kontrovers geführt. Zunehmende Zweifel daran, die biogene Elementsignatur des Knochenminerals hinreichend sicher von diagenetischen Überzeichnungen abzugrenzen sowie wiederholte Kritik hinsichtlich der prinzipiellen Effizienz des verwendeten Verfahrens zur Aufreinigung von Knochenproben wurden zum Anlaß genommen, die bisher verwandte Methodik zu überprüfen.

Zunächst wurden Spurenelementdaten früherer Untersuchungen hinsichtlich der Abhängigkeit der Konzentrationen der nahrungsanzeigenden Elemente Barium und Strontium von der histologischen Erhaltung der Knochenbinnenstruktur überprüft. Es konnten für Stichproben der Skelettpopulationen von drei alamannischen Gräberfeldern (Weingarten, Kirchheim unter Teck und Wenigumstadt) anhand der Knochenkonzentrationsdaten von insgesamt 365 Individuen diagenetisch bedingte systematische Erhöhungen der Bariumund Strontiumgehalte nachgewiesen werden. Diese Ergebnisse und die Tatsache, daß die überprüften Konzentrationen in Proben gemessen wurden, die entsprechend der wissenschaftlich akzeptierten chemischen Aufreinigungsmethode vorbereitet waren, erforderte eine experimentelle Überprüfung des Verfahrens.

Anhand einer Stichprobe von 48 Knochenproben eines weiteren alamannischen Gräberfeldes (Kirchheim am Ries) wurde die Effizienz von mechanischer Oberflächenbehandlung und chemischer Aufreinigung überprüft. Die Elementkonzentrationen in Knochenproben vor und nach der Behandlung wurden verglichen. Es konnte für keine der verwendeten Methoden ein hinreichender Aufreinigungseffekt festgestellt werden. Insbesondere muß die allgemein akzeptierte chemische Aufreinigung als ineffizient zur Beseitigung diagenetischer Konzentrationserhöhungen der nahrungsanzeigenden Elemente Barium und Strontium bewertet werden. 
Aus der Evidenz diagenetischer Konzentrationserhöhungen der nahrungsanzeigenden Elemente und der Irreversibilität dieser das Nahrungssignal verfälschenden Veränderungen leitet sich die Erfordernis einer erweiterten Diagenesecharakterisierung ab.

Eine Diagnose diagenetischer Veränderungen des Knochenminerals erfolgte über chemische Eigenschaften des nativen Knochenminerals, die als Kontrollkriterien verwendet werden können. Als ein solches Kriterium wurde auch der Zusammenhang zwischen den Barium- und Strontiumkonzentrationen überprüft, der aus der spezifischen Ernährungsabhängigkeit der beiden Elemente resultieren soll. Den Schwerpunkt der Auswertungen zur Diagenesecharakterisierung bildeten jedoch knochenimmanente Elemente (Calcium, Phosphor, Magnesium, Natrium), deren Konzentrationen ernährungsunabhängig, d.h. nur mineralchemisch, bedingt sind oder unter physiologischer Kontrolle stehen. Deren Konzentrationen bzw. charakteristische Konzentrationsverhältnisse wurden als Anzeiger für natives Knochenmineral bzw. bei Abweichungen in den bodengelagerten Knochen als Indikatoren diagenetischer Veränderungen ausgewertet. Außerdem wurde das im nativen Knochen nur in sehr geringen Konzentrationen enthaltene Aluminium als Kontaminationsanzeiger analysiert. Als weitere von den elementanalytischen Daten unabhängige Kontrollkriterien wurden charakteristische Masseverluste der Knochensubstanz während der Probenvorbereitung einschließlich der Probenaufreinigung geprüft.

Nach den Resultaten dieser Untersuchungen ermöglichen die elementanalytischen Kontrollvariablen nur eine Abschätzung des Vorhandenseins diagenetischer Veränderungen. Eine Quantifizierung erscheint infolge der hohen Variabilität der Ausprägung der Veränderungen fraglich. Insbesondere muß aber festgestellt werden, daß sich das CalciumPhosphor-Verhältnis sowie der Strontium-Barium-Zusammenhang für eine Diagenesecharakterisierung als untauglich erwiesen haben.

Für ein erweitertes Verständnis diagenetischer Veränderungen des Knochenminerals wurden die pauschalanalytischen Konzentrationsvergleiche zwischen verschiedenen Knochenproben durch mikroanalytische Untersuchungen einer Einzelprobe ergänzt. Für eine Knochenprobe, die innerhalb ihrer Kompakta unterschiedliche Grade diagenetischer Veränderungen der Knochenbinnenstruktur aufwies, konnten für die knochenimmanten Elemente Calcium, Phosphor, Magnesium, Natrium sowie Schwefel vom Erhaltungszustand der Knochenstruktur abhängige Verteilungsmuster dargestellt werden. Aus diesen Verteilungsmustern wird u.a. das Phänomen des gegenüber diagenetischen Veränderungen indifferenten Calcium-Phosphor-Verhältnisses besonders deutlich. 
Die Ergebnisse der Mikroanalysen wurden kombiniert mit den Auswertungen der pauschalanalytischen Untersuchungen $\mathrm{zu}$ den diagenesecharakterisierenden Kontrollkriterien interpretiert. Es erfolgte eine Diskussion im Kontext der in der Literatur vorhandenen Erklärungsansätze zu diagenetischen Konzentrationsveränderungen. Ein Aspekt ist hierbei die Frage nach der Ursache der Ineffizienz der wissenschaftlich akzeptierten chemischen Aufreinigung.

Als eigenständiger, von der Diageneseproblematik unabhängiger, Untersuchungsteil wurde die intraossäre Variabilität des ernährungsanzeigenden Elementes Strontium untersucht. Es konnte für dieses Element eine systematische Verteilung innerhalb einer Femurdiaphyse festgestellt werden. Hieraus kann auf eine knochenphysiologisch lokal beeinflußte Inkorporation von Strontium geschlossen werden. Dies wurde als ein weiteres Problem für Interpretationen der Knochenkonzentrationen von Strontium als Ernährungssignal diskutiert. 
Akritas MG, Arnold SF, Brunner E (1997) Nonparametric hypotheses and rank statistics for unbalanced factorial designs. J Am Stat Assoc 92: 258-265

Amtmann E, Doden E (1981) Anpassung der Knochenstruktur an mechanische Beanspruchung. Z Morph Anthrop 72: 1-21

Amtmann E, Schmitt HP (1968) Über die Verteilung der Corticaldichte im menschlichen Femurschaft und ihre Bedeutung für Bestimmung der Knochenfestigkeit. Z Anat Entwicklungsgesch 127: 25-41

Armstrong JT (1991) Quantitative element analysis of individual microparticles with electron beam instruments. In: Heinrich KFJ, Newbury DE (eds) Electron probe quantitation. Plenum Press, New York, pp 261-315

Banner JL (1995) Application of the trace-element and isotope geochemistry of strontium to studies of carbonate diagenesis. Sedimentology 42: 805-824

Becker I (1985) Zur Konstitution der frühgeschichtlichen Bevölkerung von Kirchheim unter Teck. Diplomarbeit, Universität Ulm

Bigi A, Cojazzi G, Panzavolta S, Ripamonti A, Roveri N, Romanello M, Noris SK, Moro L (1997) Chemical and structural characterization of the mineral phase from cortical and trabecular bone. J Inorg Biochem 68: 45-51

Bilezikian JP, Raisz LG, Rodan GA (eds) (1996) Principles of bone biology. Academic Press, San Diego London Boston

Blakely RL (1989) Bone strontium in pregnant and lactating females from archaeological samples. Am J Phys Anthropol 80: 173-185

Blakely RL, Beck LA (1981) Trace elements, nutritional status, and social stratification at Etowah, Georgia. Ann N Y Acad Sci 376: 417-431

Bortz J (1999) Lehrbuch der Statistik. Für Sozialwissenschaftler. 5. Aufl. Springer, Berlin Heidelberg New York

Bortz J, Lienert GA, Boehnke K (2000) Verteilungsfreie Methoden in der Biostatistik. 2. Aufl. Springer, Berlin Heidelberg New York

Brätter P, Gawlik D, Lausch J, Rösick U (1977) On the distribution of trace elements in the human skeletons. J Radioanalyt Chem 37: 393-403

Brunner E, Puri ML (2001) Nonparametric methods in factorial designs. Statistical Papers 42: 1-52

Budd P, Montgomery J, Barreiro B, Thomas RG (2000) Differential diagenesis of strontium in archaeological human dental tissues. Appl Geochem 15: 687-694

Buikstra JE, Frankenberg S, Lambert JB, Xue L (1989) Multiple elements: multiple expectations. In: Price TD (ed) The chemistry of prehistoric human bone. School of american research advanced seminar series. 1. edn. Cambridge University Press, Cambridge New York, pp $155-210$

Burton JH, Price TD (1990a) Paleodietary applications of barium values in bone. In: Pernicka E, Wagner GA (eds) Archaeometry '90. Birkhäuser, Basel, pp 787-795

Burton JH, Price TD (1990b) The ratio of barium to strontium as a paleodietary indicator of consumption of marine resources. J Arch Sci 17: 547-557

Burton JH, Price TD, Middelton WD (1999) Correlation of bone $\mathrm{Ba} / \mathrm{Ca}$ and $\mathrm{Sr} / \mathrm{Ca}$ due to biological purification of calcium. J Arch Sci 26: 609-616 
Cazalbou S, Combes C, Rey C (2000) Biomimetic approach for strontium-containing Ca-P bioceramics with enhanced biological activity. Bioceramics 192-1: 147-150

Chhettry A, Wang Z, Hsu J, Fox JL, Baig AA, Barry AM, Zhuang H, Otsuka M, Higuchi WI (1999) Metastable equilibrium solubility distribution of carbonated apatite as a function of solution composition. J Colloid Interface Sci 218: 47-56

Chickerur NS, Tung MS, Brown WE (1980) A mechanism for incorporation of carbonate into apatite. Calcif Tissue Int 32: 55-62

Child AM (1995) Towards an understanding of the microbial decomposition of archaeological bone in the burial environment. J Arch Sci 22: 165-174

Christlein R (1991) Die Alamannen. Archäologie eines lebendigen Volkes. 3. Aufl. Theiss, Stuttgart Aalen

Comar CL, Russel RS, Wasserman RH (1957) Strontium-Calcium movement from soil to man. Science 126: 485-492

D'Haese PC, Couttenye MM, Lamberts LV, Elseviers MM, Goodman WG, Schrooten I, Cabrera WE, De Broe ME (1999) Aluminum, iron, lead, cadmium, copper, zinc, chromium, magnesium, strontium, and calcium content in bone of end-stage renal failure patients. Clin Chem 45: 1548-1556

Dorozhkin SV (1997) Surface reactions of apatite dissolution. J Colloid Interface Sci 191: 489-497

Driessens FC (1980) Probable phase composition of the mineral in bone. Z Naturforsch [C] 35: 357-362

Driessens FC, Verbeeck RM (1980) Evidence for intermediate metastable states during equilibration of bone and dental tissues. Z Naturforsch [C] 35: 262-267

Edward JB, Benfer RA, Morris JS (1990) The effects of dry ashing on the composition of human and animal bone. Biol Trace Elem Res 25: 219-231

Elias RW, Croxdale J (1980) Investigations of the deposition of lead-bearing aerosol on the surfaces of vegetation. Sci Total Environ 14: 265-278

Elias RW, Hirao Y, Patterson CC (1982) The circumvention of the natural biopurification of calcium along nutrient pathways by atmospheric inputs of industrial lead. Geochim Cosmochim Acta 46: 2561-2580

Elliott JC (1994) Structure and chemistry of the apatites and other calcium orthophosphates. Elsevier, Amsterdam London New York

Embery G, Rolla G (1980) Interaction between sulphated macromolecules and hydroxyapatite studied by infrared spectroscopy. Acta Odontol Scand 38: 105-108

Ezzo JA (1994) Putting the "chemistry" back into archaeological bone chemistry analysis: modeling potential paleodietary indicators. J Anthropol Arch 13: 1-34

Fabig A, Schutkowski H, Herrmann B (2000) Differentiation between occupational and dietaryrelated intake of barium in the skeleton. Anthropol Anz 58: 105-111

Filip J (1969) Enzyklopädisches Handbuch zur Ur- und Frühgeschichte Europas. Band II (L-Z). W. Kohlhammer, Stuttgart Berlin Köln

Francalacci P, Borgognini Tarli S (1988) Multielementary analysis of trace elements and preliminary results on stable isotopes in two italian prehistoric sites. Methodological aspects. In: Grupe G, Herrmann B (eds) Trace elements in environmental history. Springer, Berlin Heidelberg New York, pp 41-52

Gawlik D, Behne D, Brätter P, Gatschke W, Gessner H, Kraft D (1982) The suitability of the iliac crest biobsy in the element analysis of bone and marrow. J Clin Chem Clin Biochem 20: 499-507 
Gilbert C, Sealy J, Sillen A (1994) An investigation of barium, calcium and strontium as palaeodietary indicators in the southwestern Cape, South Africa. J Arch Sci 21: 173-184

Gordon CC, Buikstra JE (1981) Soil pH, bone preservation, and sampling bias at mortuary sites. Amer Antiq 46: 566-571

Grupe G (1988) Impact of the choice of bone samples on trace element data in excavated human skeletons. J Arch Sci 15: 123-129

Grupe G, Dreses-Werringloer U, Parscha F (1993) Initial stages of bone decomposition: causes and consequences. In: Lambert JB, Grupe G (eds) Prehistoric human bone - Archaeology at the molecular level. Springer, Berlin Heidelberg New York, pp 257-274

Grupe G, Piepenbrink H (1988) Trace element contaminations in excaveted bones by microorganisms. In: Grupe G, Herrmann B (eds) Trace elements in environmental history. Springer, Berlin Heidelberg New York, pp 104-112

Grupe G, Schutkowski H (1989) Dietary shift during the 2nd millenium BC at prehistoric Shimal, Oman peninsula. Paléorient 15: 77-84

Grynpas M (1993) Age and disease-related changes in the mineral of bone. Calcif Tissue Int 53 Suppl 1: S57-S64

Hancock RGV, Grynpas MD, Pritzker KPH (1989) The abuse of bone analyses for archaeological dietary studies. Archaeometry 31: 169-179

Hanson DB, Buikstra JE (1987) Histomorphological alteration in buried human bone from the lower Illinois Valley: implications for paleodietary research. J Arch Sci 14: 549-563

Hedges REM, Millard AR (1995) Bones and groundwater: towards the modelling of diagenetic processes. J Arch Sci 22: 155-164

Hedges REM, Millard AR, Pike AWG (1995) Measurements and relationships of diagenetic alteration of bone from three archaeological sites. J Arch Sci 22: 201-209

Herrmann B (2001) Zwischen Molekularbiologie und Mikrohistorie. Vom Ort der Historischen Anthropologie. Jahrbuch 2000 der Deutschen Akademie der Naturforscher Leopoldina. LEOPOLDINA (R. 3). vol. 46 Halle/Saale, pp 391-408

Herrmann B, Newesely H (1982) Dekompositionsvorgänge des Knochens unter langer Liegezeit. 1. Die mineralische Phase. Anthropol Anz 40: 19-31

Herrmann B, Nitsch KH (1984) Ein spezifisches Calciumphosphat und die Erfassung kleinräumiger Liegemileubedingungen in einer frühmittelalterlichen Grabanlage von Köniz, Kanton Bern. Archives suisses d'anthropologie générale 48: 65-68

Jackson KW, Mahmood TM (1994) Atomic absorption, atomic emission, and flame emission spectrometry. Anal Chem 66: 252R-279R

Jarvis I, Jarvis KE (1992) Plasma spectrometry in the earth sciences: techniques, applications and future trends. Chem Geol 95: 33

Katzenberg MA (1984) Chemical analysis of prehistoric human bone from five temporally distinct populations in southern Ontario. National Museum of Man, Mercury Series, Archaeological Survey of Canada, Paper 129.

Klepinger LL, Kuhn JK, Williams WS (1986) An elemental analysis of archaeological bone from Sicily as a test of predictability of diagenetic change. Am J Phys Anthropol 70: 325-331

Lambert JB, Simpson SV, Buikstra JE, Hanson D (1983) Electron microprobe analysis of elemental distribution in excavated human femurs. Am J Phys Anthropol 62: 409-423

Lambert JB, Simpson SV, Szpunar CB, Buikstra JE (1985) Bone diagenesis and dietary analysis. J Hum Evol 14: 477-482 
Lambert JB, Szpunar CB, Buikstra JE (1979) Chemical analysis of excavated human bone from middle and late woodland sites. Archaeometry 21: 115-129

Lambert JB, Vlasak SM, Thometz AC, Buikstra JE (1982) A comparative study of the chemical analysis of ribs and femurs in Woodland populations. Am J Phys Anthropol 59: 289-294

Lambert JB, Weydert-Homeyer JM (1993) The fundamental relationship between ancient diet and the inorganic constituents of bone as derived from feeding experiments. Archaeometry 35: 279-294

Lambert JB, Xue L, Buikstra JE (1989) Physical removal of contaminative inorganic material from buried human bone. J Arch Sci 16: 427-436

Leblond CP, Weinstock M (1976) A comparative study of dentin and formation. In: Bourne GH (ed) The biochemistry and physilology of bone. Academic Press, New York, pp 517-562

Lee AP, Klinowski J, Marseglia EA (1995) Application of nuclear magnetic resonance spectroscopy to bone diagenesis. J Arch Sci 22: 257-262

LeGeros RZ, Kijkowska R, Bautista C, LeGeros JP (1995) Synergistic effects of magnesium and carbonate on properties of biological and synthetic apatites. Connect Tissue Res 33: 203209

McFarren EF, Lishka TRJ, Parker JH (1970) Criterion for judging acceptability of analytical methods. Anal Chem 42: 358-365

Microsoft, Corp. (1999) EXCEL 2000 [Computerprogramm]. Microsoft Corp.

Millard AR, Hedges REM (1995) The role of the environment in uranium uptake by buried bone. J Arch Sci 22: 239-250

Mongiorgi R, Gnudi S, Moroni A, Bertocchi G, Galliani I, Benfenati L (1990) Bone mineral alterations and $\mathrm{Mg}$ content in aging. Boll Soc Ital Biol Sper 66: 623-630

Nelson BK, DeNiro MJ, Schoeninger MJ, DePaolo DJ, Hare PE (1986) Effects of diagenesis on strontium carbon nitrogen and oxygen concentration and isotopic composition of bone. Geochim Cosmochim Acta 50: 1941-1949

Nelson DG (1981) The influence of carbonate on the atomic structure and reactivity of hydroxyapatite. J Dent Res 60: 1621-1629

Nelson DG, Featherstone JD, Duncan JF, Cutress TW (1982) Paracrystalline disorder of biological and synthetic carbonate-substituted apatites. J Dent Res 61: 1274-1281

Neuffer-Müller C (1983) Der alamannische Adelsbestattungsplatz und die Reihengräberfriedhöfe von Kirchheim am Ries (Ostalbkreis). Forschungen und Berichte zur Vor- und Frühgeschichte in Baden-Württemberg. (Bd. 15) Theiss, Stuttgart

Nielsen-Marsh CM, Hedges REM (1999) Bone porosity and the use of mercury intrusion porosimetry in bone diagenesis studies. Archaeometry 41: 165-174

Nielsen-Marsh CM, Hedges REM (2000a) Patterns of diagenesis in bone I: the effects of site environments. J Arch Sci 27: 1139-1150

Nielsen-Marsh CM, Hedges REM (2000b) Patterns of diagenesis in bone II: effects of acetic acid treatment and the removal of diagenetic $\mathrm{CO}_{3}{ }^{2-}$. J Arch Sci 27: 1151-1159

Parker RB, Toots H (1970) Minor elements in fossil bone. Geological Society of America Bulletin 81: 925-932

Parker RB, Toots H (1980) Trace elements in bones as paleobiological indicators. In: Behrensmeyer AK, Hill AP (eds) Fossils in the making: Vertebrate taphonomy and paleoecology. University Press, Chicago, pp 197-207

Pate FD (1994) Bone chemistry and paleodiet. J Archaeol Method Theory 1: 161-209 
Pate FD, Brown KA (1985) The stability of bone strontium in the geochemical environment. J Hum Evol 14: 483-491

Pate FD, Hutton JT (1988) The use of soil chemistry data to address post-mortem diagenesis in bone mineral. J Arch Sci 15: 729-739

Pate FD, Hutton JT, Gould RA, Pretty GL (1991) Alterations of in-vivo elemental dietary signatures in archaeological bone evidence from the roonka flat dune South Australia, Australia. Archaeol Oceania 26: 58-69

Pate FD, Hutton JT, Norrish K (1989) Ionic exchange between soil solution and bone: toward a predictive model. Appl Geochem 4: 303-316

Penel G, Leroy G, Rey C, Bres E (1998) MicroRaman spectral study of the $\mathrm{PO}_{4}$ and $\mathrm{CO}_{3}$ vibrational modes in synthetic and biological apatites. Calcif Tissue Int 63: 475-481

Peters F, Schwarz K, Epple M (2000) The structure of bone studied with synchrotron X-ray diffraction, X-ray absorption spectroscopy and thermal analysis. Thermochimica Acta 361: 131138

Pfeiffer S, Varney TL (2000) Quantifying histological and chemical preservation in archaeological bone. In: Ambrose SH, Katzenberg MA (eds) Biochemical approaches to paleodietary analysis. Advances in archaeological and museum science. Kluwer Academic / Plenum Puplishers, New York Boston Dordrecht, pp 141-158

Popper KR (1993) Objektive Erkenntnis: ein evolutionärer Entwurf. Campe Paperback. 1. Aufl. Hoffmann und Campe, Hamburg

Posner AS (1978) "The chemistry of bone mineral". Bull Hosp Joint Dis 39: 126-144

Price TD (1989) Multi-element studies of diagenesis in prehistoric bone. In: Price TD (ed) The chemistry of prehistoric human bone. School of american research advanced seminar series. 1. edn. Cambridge University Press, Cambridge New York, pp 126-154

Price TD, Blitz J, Burton JH, Ezzo JA (1992) Diagenesis in prehistoric bone: problems and solutions. J Arch Sci 19: 513-529

Quattropani L, Charlet L, de-Lumley H, Menu M (1999) Early Palaeolithic bone diagenesis in the Arago cave at Tautavel, France. Mineral. Mag. 63: 801-812

Radosevich SC (1993) The six deadly sins of trace element analysis. A case of wishful thinking in science. In: Sandford MK (ed) Investigations of ancient human tissue. Chemical analyses in anthropology. Food and nutrition in history and anthropology. vol. 10 Gordon and Breach Science Publishers, Langhorne, pp 269-332

Reiche I, Favre-Quattropani L., Calligaro T, Salomon J, Bocherens H, Charlet L, Menu M (1999) Trace element composition of archaeological bones and postmortem alteration in the burial environment. Nucl Instr and Meth B 150: 656-662

Roth H, Theune C (1995) Das frühmittelalterliche Gräberfeld von Weingarten. 1. Katalog der Grabfunde. Theiss, Stuttgart

Runia LT (1988) Discrimination factors on different trophic levels in relation to the trace element content in human bones. In: Grupe G, Herrmann B (eds) Trace elements in environmental history. Springer, Berlin Heidelberg New York, pp 53-66

Sandford MK, Weaver DS (2000) Trace element research in anthropology: new perspectives and challenges. In: Katzenberg MA, Saunders SR (eds) Biological anthropology of the human skeleton. Wiley-Liss, New York Chichester Weinheim, pp 329-350

SAS Institute, Inc. (2000) SAS 8.0 [Computerprogramm]. Cary, NC: SAS Institute Inc.

Schoeninger MJ (1979) Diet and status at Chalcatzingo: some empirical and technical aspects of strontium analysis. Am J Phys Anthropol 51: 295-310 
Schoeninger MJ (1989) Reconstructing prehistoric human diet. In: Price TD (ed) The chemistry of prehistoric human bone. School of american research advanced seminar series. 1. edn. Cambridge University Press, Cambridge New York,

Schoeninger MJ, Moore KM, Murray ML, Kingston JD (1989) Detection of bone preservation in archaeological and fossil samples. Appl Geochem 4: 281-292

Schug H (1997) Intraindividuelle Elementverteilung im distalen Femur. Anthropol Anz 55: 167178

Schutkowski H (1994) Spurenelementanalysen. In: Herrmann B (ed) Archäometrie. Naturwissenschaftliche Analyse von Sachüberresten. Springer, Berlin Heidelberg New York, pp 67-86

Schutkowski H (1995) What you are makes you eat different things - interrelations of diet, status, and sex in the early medieval population of Kirchheim unter Teck, FRG. Hum Evol 10: $119-130$

Schutkowski H (1998) Biokulturelle Anpassungen in menschlichen Bevölkerungen. Beiträge zu einer Humanökologie. Habilitationsschrift, Universität Göttingen

Schutkowski H, Herrmann B (1996) Geographical variation of subsistence strategies in early mediaeval populations of Southwestern Germany. J Arch Sci 23: 823-831

Schutkowski H, Wiedemann F, Bocherens H, Grupe G, Herrmann B (1999) Diet status and decomposition at Weingarten. Trace element and isotope analysis on early mediaeval skeleton material. J Arch Sci 26: 675-685

Scott VD, Love G, Reed SJB (1995) Quantitative Electron-Probe Microanalysis. Ellis Horwood series in physics and its applications. 2. edn. Horwood, New York

Sealy J (2001) Body tissue chemistry and paleodiet. In: Brothwell DR, Pollard AM (eds) Archaeological sciences. John Wiley \& Sons, Chichester New York Weinheim, pp 269-279

Shellis RP, Lee AR, Wilson RM (1999) Observations on the apparent solubility of carbonateapatites. J Colloid Interface Sci 218: 351-358

Siegel S (1987) Nichtparametrische statistische Methoden. Fachbuchhandlung für Psychologie, Eschborn bei Frankfurt am Main

Sillen A (1981) Strontium and diet at Hayonim Cave. Am J Phys Anthropol 56: 131-137

Sillen A (1986) Biogenic and diagenetic $\mathrm{Sr} / \mathrm{Ca}$ in Plio-pleistocene fossils of the Omo Shungura Formation. Paleobiology 12: 311-323

Sillen A (1989) Diagenesis of the inorganic phase of cortical bone. In: Price TD (ed) The chemistry of prehistoric human bone. School of american research advanced seminar series. 1. edn. Cambridge University Press, Cambridge New York, pp 211-229

Sillen A, LeGeros R (1991) Solubility profiles of synthetic apatites and of modern and fossil bones. J Arch Sci 18: 385-397

Sillen A, Sealy JC (1995) Diagenesis of strontium in fossil bone: a reconsideration of Nelson et al. (1986). J Arch Sci 22: 313-320

Sillen A, Smith P (1984) Weaning patterns are reflected in strontium-calcium ratios of juvenile skeletons. J Arch Sci 11: 237-245

Simon K, Wiechert U, Hoefs J, Grote B (1997) Microanalysis of minerals by laser ablation ICPMS and SIRMS. Fresenius J Anal Chem 359: 458-461

Skoog DA, Leary JJ (1996) Instrumentelle Analytik: Grundlagen - Geräte - Anwendungen. Springer, Berlin Heidelberg New York 
Stark G (1968) Untersuchungen an synthetischem Hydroxylapatit im Hinblick auf den Knochenstoffwechsel von Calcium, Strontium, Barium und Radium. I. Die Diskriminierung gegen Calcium. Biophysik 5: 42-54

StatSoft, Inc. (1998) STATISTICA für Windows 5.1 [Computerprogramm]. Tulsa, OK: StatSoft

StatSoft, Inc. (2001) Electronic Statistics Textbook. Tulsa, OK: StatSoft. URL: http://www.statsoft.com/textbook/stathome.html

Stauch E (2000) Wenigumstadt - Ein Bestattungsplatz der Völkerwanderungszeit und des frühen Mittelalters im nördlichen Odenwaldvorland. Archäol Nachr 5: 332-334

Tanaka G, Kawamura H, Nomura E (1981) Reference japanese man - II Distribution of strontium in the skeleton and in the mass of mineralized bone. Health Physics 40: 601-614

Thompson J (2000) Robust, resistant and nonparametric methods. In: Tranter RL (ed) Design and analysis in chemical research. Sheffield analytical chemistry. Sheffield Academic/CRC Press, Boca Raton, Fla, pp 145-187

Toots H, Voorhies MR (1965) Strontium in fossil bones and the reconstructions of food chains. Science 149: 854-855

Tuross N, Behrensmeyer AK, Eanes ED (1989) Strontium increases and crystallinity changes in taphonomic and archaeological bone. J Arch Sci 16: 661-672

Utsumi M, Tohno S, Minami T, Okazaki Y, Moriwake Y, Yamada M, Tohno Y (1999) Ageindependent constancy of mineral contents in human ribs. Biol Trace Elem Res 67: 165171

Welz B (1997) Atomabsorptionsspektrometrie. 4. Aufl. Wiley-VCH, Weinheim-Basel

White EM, Hannus LA (1983) Chemical weathering of bone in archaeological soils. Amer Antiq 48: 316-322

Williams CT (1988) Alteration of chemical composition of fossil bones by soil processes and groundwater. In: Grupe G, Herrmann B (eds) Trace elements in environmental history. Springer, Berlin Heidelberg New York, pp 27-40

Williams CT, Henderson P, Marlow CA, Molleson TI (1997) The environment of deposition indicated by the distribution of rare earth elements in fossil bones from Olduvai Gorge, Tanzania. Appl Geochem 12: 537-547

Wright LE, Schwarcz HP (1996) Infrared and isotopic evidence for diagenesis of bone apatite at Dos Pilas, Guatemala: palaeodietary implications. J Arch Sci 23: 933-944 


\section{$8 \quad$ Anhang}

\subsection{Verwendete Abkürzungen}

\section{Abkürzungen}

AA

AAS

ANOVA

Aqua bidest.

ASCII

EDS

EPMA

FAAS

FEP

ICP-OES

LA-ICP-TOFMS

LDPE

PMP

PP

RT

SAS/IML

SEM

WDS
Atomabsorption (als Meßtechnik)

Atomic Absorptions Spectrometry

Analysis of Variance

destilliertes demineralisiertes Wasser

American Standard Code for Information Interchange

Energy Dispersive Spectrometry (bzw. Spectrometer)

Electron Probe Microanalysis (bzw. Electron Probe Microanalyser)

Flame Atomic Absorption Spectrometry

Fluorinated Ethylene Propylene

Inductively Coupled Plasma Optical Emission Spectrometry

Laser Ablation Inductively Coupled Plasma Time of Flight Mass

Spectrometry

Low-Density Polyethylene

Polymethylpentene

Polypropylene

Raumtemperatur

Statistical Analysis System / Interactive Matrix Language

Scanning Electrone Microscope

Wavelength Dispersive Spectromtry (bzw. Spectrometer)

\subsection{Geräte und Chemikalien}

\section{Analyse-Technik}

AAS 1100B Perkin ElmerTM

EPMA (hier Electron probe microanalyzer) JXA 8900 RL, JEOL

ICP-OES OPTIMA $3300^{\mathrm{TM}}$ DV, Perkin Elmer ${ }^{\mathrm{TM}}$

LA-ICP-TOFMS:

- Excimer Laser, COMPex $^{\mathrm{TM}}$ 110, Lambda Physik ${ }^{\circledR}$ (ArF, 193 nm)

- Optische Bank GeoLas 100 Q, MicroLas Lasersystem

- ICP- TOFMS, LECO Renaissance ${ }^{\mathrm{TM}}$

Photometer Eppendorf ${ }^{\circledR} 1101 \mathrm{M}$

\section{Kleingeräte, Laborzubehör und Hilfsmittel}

Apparatur für Ätherextraktion:

- Extraktor nach Soxhlet, $100 \mathrm{ml}$

- Rundkolben, $250 \mathrm{ml}$

- Kühler nach Dimroth, 250 mm Hantel

- $\quad$ PILZ $^{\circledR}$ Standardheizhaube, Typ G2

Aufschlußapparatur A6×100, (mit Quarzglaseinsätzen, 10 ml), Seif-Aufschlusstechnik Bandsäge, T1, Black \& Decker ${ }^{\circledR}$ 
Bechgläser, Duran ${ }^{\circledR}$, Schott (niedrige Form, $5000 \mathrm{ml}$ mit Glasgittereinsatz zum Ausdämpfen; weitere in niedriger und hoher Form, verschiedene Größen)

CCD-Kamera-System (Auflösung 2048×2048), Stemmer

Ceramic-Messer, Heinr. Böker Baumwerk, $(9,3 \mathrm{~cm})$

Deckgläser $24 \times 32 \mathrm{~mm}$, IDL

Dentalmikromotor K-10, Handstück und Steuergerät, KaVo-EWL

(Sägeblätter: Diamantscheiben H 350 220, Horico, Diamant Instrumente, Hopf. Ringleb \& Co.)

Eppendorf Reaktionsgefäße, safe-lock, Eppendorf $(2,0 \mathrm{ml})$

Exsikkator, Glaswerk Wertheim

Fix Volumenmikroliter-Pipette, Reference ${ }^{\circledR}$, Eppendorf

Gefäße für Stamm- und Meßlösungen sowie für Verdünnungsmedien:

- Enghalsflaschen, Kautex, aus LDPE, (10 ml, 1000 ml, $2000 \mathrm{ml})$

- Enghalsflaschen, Nalgene ${ }^{\circledR}$, IDL, aus Teflon FEP (30 ml, $\left.60 \mathrm{ml} ; 125 \mathrm{ml}\right)$

- Rollrand-Schnappdeckelgläser, ScherfGlas ${ }^{\circledR},($ ca. $30 \mathrm{ml})$

- Spritzflaschen, Nalgene ${ }^{\circledR}$, IDL, aus LDPE (500 ml)

- Vorratsflaschen, Enghals aus HDPE, Kautex ${ }^{\circledR},(10$ l)

- Weithalsflaschen, Nalgene ${ }^{\circledR}$, aus PP, $(1000 \mathrm{ml})$

Glasspatel, $8 \mathrm{~mm}$ Rohr, $150 \mathrm{~mm}$, Sonderanfertigung, Rettberg

Halbanalysenwaage R 160 P, Sartorius ${ }^{\circledR}$

Handdispenser Multipette ${ }^{\circledR}$ plus, Eppendorf

Innenlochsäge Leitz ${ }^{\circledR} 1600$

Kimwipes ${ }^{\circledR}$ Präzisionswischtücher, Kimberly Clark ${ }^{\circledR}($ small, $22 \times 11,5 \mathrm{~cm})$

Laminar-Air-Flow-System, Schymura Maschinen-Gerätebau

Lichtmikroskop Olympus ${ }^{\circledR}$ BH-2

Magnetrührer/Heizgerät MR 3001 Heidolph

Meßkolben, Duran ${ }^{\circledR}$, meist Hirschmann ${ }^{\circledR}$ oder Brand, aber auch andere Hersteller (verschiedene Größen)

Meßpipette, Blaubrand ${ }^{\circledR}$, Brand, $5 \mathrm{ml}$

Meßzyliner, Duran ${ }^{\circledR}$ verschiedene Hersteller, (verschiedene Größen)

Mikroschaufel, 18/8-Stahl, teflonisiert, Bochem

Mörser, Achat mit Pistill, C. Giese KG, (I.- $\varnothing$ ca. 100 mm)

Muffelofen, Heraeus ${ }^{\circledR}$ KM 260

Objektträger, ca. $76 \times 26 \mathrm{~mm}$; IDL

Parafilm $\mathrm{M}^{\mathbb{B}}$, American National Can ${ }^{\mathrm{TM}}$

Pinzetten:

- Fine Ceramic Tweezer 8501, Vomm Solingen

- aus PMP, VIT-LAB

Pipettierhilfe pipetus ${ }^{\circledR}$ akku, Hirschmann ${ }^{\circledR}$

Schneidmaschine, Typ 270109, Bodo Schmidt, Göttingen

Skalpell: -griff, (135 mm), Hammacher und auswechselbare Klingen, spitz (35 mm)

Trockenschrank, Heraeus ${ }^{\circledR}$

Trockenschrank, Memmert

Ultraschallbad Sonorex RK 102, Bandelin electronic

Variable Mikroliterpipetten Reference ${ }^{\circledR}$, Eppendorf (10-100 $\left.\mu 1,50-250 \mu 1,500-2500 \mu 1\right)$

Vollpipetten Blaubrand $^{\circledR}$, Brand (1 ml, $\left.9 \mathrm{ml}\right)$

Wägepapier, MN 226, Macherey-Nagel $(9 \times 11,5 \mathrm{~cm})$ 


\section{Chemikalien}

Brenngase für AAS:

Acetylen 2.6, $\mathrm{C}_{2} \mathrm{H}_{2}$, Messer Griesheim

Distickstoffmonoxyd pro narcosi, $\mathrm{N}_{2} \mathrm{O}$, GHC Gerling, Holz \& Co. Handels Plasma-/Probentransportgas für ICP-OES und ICP-TOFMS

Argon 4.0

Ameisensäure 98-100 \%, zur Analyse, ACS, HCOOH, Merck KGaA, Darmstadt

Barium-Standardlösung, $1000 \mathrm{mg} / \mathrm{Ba}\left(\mathrm{NO}_{3}\right)_{2}$ in $\mathrm{HNO}_{3}$ 0,5 mol/1, Merck KGaA, Darmstadt

Biodur $^{\mathrm{TM}}$ (Standardepoxidharz E12 und Härter E1), Biodur Products

Calcium-Standardlösung, $1000 \mathrm{mg} / 1 \mathrm{Ca}\left(\mathrm{NO}_{3}\right)_{2}$ in $\mathrm{HNO}_{3}$ 0,5 mol/1, Merck KGaA, Darmstadt

Diethylether, reinst, $\left(\mathrm{C}_{2} \mathrm{H}_{5}\right)_{2} \mathrm{O}$, E. Merck, Darmstadt

Ethanol 99,8\%, Sorte 510, Neutralalkohol, entwässert

Eukitt, Einschlußmittel für mikroskopische Technik, O. Kindler \& Co.

Kaliumchlorid, zur Analyse, KCl, E. Merck, Darmstadt

Lanthan(III)Oxid, (max. 0,0005\% Ca; max. 0,002\% $\mathrm{Pb}$ ) für die Atomabsorptionsspektroskopie, $\mathrm{La}_{2} \mathrm{O}_{3}$, Merck, Darmstadt

Multielementstandardlösung, 7 Elemente (in Salpeterlösung, etwa $2 \mathrm{~mol} / \mathrm{l}$ ), Bernd Kraft Salze \& Lösungen, (Konzentrationen: 500 mg/l Ti; 5000 mg/l Al, Ca, Fe, K, Mg, Na)

Multielementstandardlösung, 20 Elemente (in Salpeterlösung, etwa $2 \mathrm{~mol} / \mathrm{l}$ ), Bernd Kraft Salze \& Lösungen, (Konzentrationen: 10 mg/l As, Be, La, Mo, Pb, Sc, Y; 100 mg/l Al, $\mathrm{Ba}, \mathrm{Co}, \mathrm{Cr}, \mathrm{Cu}, \mathrm{Fe}, \mathrm{Li}, \mathrm{Mn}, \mathrm{Ni}, \mathrm{Sr}, \mathrm{V}, \mathrm{Zn}, \mathrm{Zr}$ )

Natriummolybdat-Dihydrat, zur Analyse, $\mathrm{Na}_{2} \mathrm{MO}_{4} \times 2 \mathrm{H}_{2} \mathrm{O}$, E. Merck, Darmstadt

Salpetersäure $65 \%, \mathrm{HNO}_{3}$, Suprapur ${ }^{\circledR}$, Merck, Darmstadt

SRM 610, Standard Reference Material 610 - Silicate Glass, National Institute of Standards \& Technology, Gaithersburg

SRM 1400, Standard Reference Material 1400 - Bone Ash, National Institute of Standards \& Technology, Gaithersburg

Standard Animal Bone H-5, International Atomic Energy Agency, Wien

Strontium-Standardlösung, $1000 \mathrm{mg} / \mathrm{S} \mathrm{Sr}\left(\mathrm{NO}_{3}\right)_{2}$ in $\mathrm{HNO}_{3}$ 0,5 mol/l, Merck, Darmstadt 


\subsection{Geräte- und Analyseparameter}

\subsubsection{FAAS}

Tabelle 11: FAAS - Geräteparameter

\begin{tabular}{|l|c|c|c|}
\hline Parameter & Calcium & Barium & Strontium \\
\hline Technik & $\mathrm{AA}$ & $\mathrm{AA}$ & $\mathrm{AA}$ \\
Lampenstrom [mA] & 15 & 25 & 20 \\
Wellenlänge [nm] & 422,7 & 553,7 & 460,8 \\
Spaltbreite [nm] & $0,7 \mathrm{H}$ & $0,2 \mathrm{H}$ & $0,2 \mathrm{H}$ \\
signal processing & hold & hold & hold \\
Integrationszeit [s] & 2 & 2 & 2 \\
Anzahl der Wiederholungsmessungen & 3 & 3 & 3 \\
linearer Meßbereich bis [mg/l] & 5 & 10 & 5 \\
Charakteristische Konzentration [mg/l] & 0,09 & 0,5 & 0,1 \\
Brennerkopf - Schlitzlänge [cm] & 5 & 5 & 5 \\
Brenngas & $\mathrm{C}_{2} \mathrm{H}_{2}$ & $\mathrm{C}_{2} \mathrm{H}_{2}$ & $\mathrm{C}_{2} \mathrm{H}_{2}$ \\
Oxidationsgas & $\mathrm{N}_{2} \mathrm{O}$ & $\mathrm{N}_{2} \mathrm{O}$ & $\mathrm{N}_{2} \mathrm{O}$ \\
\hline
\end{tabular}

AA = Atomabsorption (ohne Background Kompensation)

$\mathrm{H} \quad=$ High; Spaltoptimierung für FAAS

Tabelle 12: FAAS - Analysebedingungen

\begin{tabular}{|c|c|c|c|c|c|c|}
\hline Element & $\begin{array}{l}\text { Verdünnungs- } \\
\text { medium }\end{array}$ & Verdünnung & Kalibrierlösung & \multicolumn{3}{|c|}{$\begin{array}{c}\text { Kalibrierung(en) } \\
{[\mathrm{mg} / \mathrm{l}]}\end{array}$} \\
\hline $\mathrm{Ca}$ & $0,1 \% \mathrm{La}_{2} \mathrm{O}_{3}{ }^{*}$ & $1: 550(1: 600)$ & Standardlsg. $+0,1 \% \mathrm{La}_{2} \mathrm{O}_{3}{ }^{*}$ & 1,0 & 2,0 & 5,0 \\
\hline $\mathrm{Sr}$ & $0,1 \% \mathrm{KCl}^{*}$ & $1: 5(1: 10)$ & Standardlsg. $+0,1 \% \mathrm{KCl}^{*}$ & 3,0 & & \\
\hline $\mathbf{B a}$ & $0,1 \% \mathrm{KCl}^{*}$ & $1: 2$ & Standardlsg. $+0,1 \% \mathrm{KCl}^{*}$ & 10,0 & & \\
\hline
\end{tabular}

${ }^{*} 0,65 \% \mathrm{HNO}_{3}$ Gehalt 


\subsubsection{ICP-OES}

Tabelle 13: ICP-OES - Geräteparameter

(Angaben geräteseitig in englischer Sprache)

\begin{tabular}{|c|c|c|c|}
\hline & Parameter & \multicolumn{2}{|c|}{ Value } \\
\hline Spectrometer & $\begin{array}{l}\text { Spectral Profiling } \\
\text { Resolution } \\
\text { Purge Gas Flow } \\
\text { Replicates } \\
\text { Read Time }\end{array}$ & $\begin{array}{l}\text { No } \\
\text { High } \\
\text { Norma } \\
2 \\
5\end{array}$ & sec \\
\hline Plasma Parameters & $\begin{array}{l}\text { Plasma } \\
\text { Auxilary } \\
\text { Nebulizer } \\
\text { Power } \\
\text { View Height } \\
\text { Plasma View }\end{array}$ & $\begin{array}{c}15 \\
0,4 \\
0,75 \\
1300 \\
15 \\
\text { Axial }\end{array}$ & $\begin{array}{l}\mathrm{I} / \mathrm{min} \\
\mathrm{I} / \mathrm{m} \\
\mathrm{I} / \mathrm{m} \\
\mathrm{W} \\
\mathrm{mm}\end{array}$ \\
\hline Pump Parameters & $\begin{array}{l}\text { Sample Flow Rate } \\
\text { Sample Flush Time } \\
\text { Sample Flush Rate } \\
\text { Read Delay Time }\end{array}$ & $\begin{array}{l}1,2 \\
5 \\
5 \\
60\end{array}$ & $\begin{array}{l}\mathrm{ml} / \mathrm{min} \\
\mathrm{sec} \\
\mathrm{ml} / \mathrm{min} \\
\mathrm{sec}\end{array}$ \\
\hline Wash Parameters & $\begin{array}{l}\text { Wash Frequency } \\
\text { Wash Rate } \\
\text { Wash Time }\end{array}$ & $\begin{array}{c}\text { Between } \\
1,2 \\
10\end{array}$ & $\begin{array}{l}\text { amples } \\
\mathrm{ml} / \mathrm{min} \\
\text { sec }\end{array}$ \\
\hline
\end{tabular}


Tabelle 14: ICP-OES - Elementspezifische Analyseparameter

\begin{tabular}{|c|c|c|c|c|}
\hline Element & Wavelength & Peak Algorithm & Pts/Peak & $\begin{array}{l}\text { Background } \\
\text { Correction }\end{array}$ \\
\hline Al & $\begin{array}{l}396,140 \\
308,211 \\
394,403\end{array}$ & $\begin{array}{l}\text { Area } \\
\text { Area } \\
\text { Area }\end{array}$ & $\begin{array}{l}1 \\
1 \\
1\end{array}$ & $\begin{array}{l}\text { 2-Point } \\
\text { 2-Point } \\
\text { 2-Point }\end{array}$ \\
\hline $\mathrm{Ba}$ & $\begin{array}{l}493,394 \\
455,389 \\
233,524\end{array}$ & $\begin{array}{l}\text { Area } \\
\text { Area } \\
\text { Area }\end{array}$ & $\begin{array}{l}2 \\
2 \\
1\end{array}$ & $\begin{array}{l}\text { 2-Point } \\
\text { 2-Point } \\
\text { 2-Point }\end{array}$ \\
\hline $\mathrm{Ca}$ & $\begin{array}{l}317,932 \\
610,267 \\
227,544 \\
315,886\end{array}$ & $\begin{array}{l}\text { Area } \\
\text { Area } \\
\text { Area } \\
\text { Area }\end{array}$ & $\begin{array}{l}2 \\
1 \\
2 \\
2\end{array}$ & $\begin{array}{l}\text { 1-Point } \\
\text { 1-Point } \\
\text { 2-Point } \\
\text { 2-Point }\end{array}$ \\
\hline Mg & $\begin{array}{l}279,073 \\
285,209 \\
202,581\end{array}$ & $\begin{array}{l}\text { Area } \\
\text { Area } \\
\text { Area }\end{array}$ & $\begin{array}{l}2 \\
1 \\
2\end{array}$ & $\begin{array}{l}\text { 2-Point } \\
\text { 1-Point } \\
\text { 1-Point }\end{array}$ \\
\hline $\mathbf{P}$ & $\begin{array}{l}214,913 \\
178,223 \\
213,618\end{array}$ & $\begin{array}{l}\text { Area } \\
\text { Area } \\
\text { Area }\end{array}$ & $\begin{array}{l}2 \\
2 \\
2\end{array}$ & $\begin{array}{l}\text { 2-Point } \\
\text { 2-Point } \\
\text { 2-Point }\end{array}$ \\
\hline $\mathrm{Sr}$ & $\begin{array}{l}460,721 \\
407,757 \\
421,536\end{array}$ & $\begin{array}{l}\text { Area } \\
\text { Area } \\
\text { Area }\end{array}$ & $\begin{array}{l}1 \\
2 \\
2\end{array}$ & $\begin{array}{l}\text { 2-Point } \\
\text { 2-Point } \\
\text { 2-Point }\end{array}$ \\
\hline $\mathrm{Na}$ & $\begin{array}{l}330,237 \\
589,562\end{array}$ & $\begin{array}{l}\text { Area } \\
\text { Area }\end{array}$ & $\begin{array}{l}2 \\
1\end{array}$ & $\begin{array}{l}\text { 2-Point } \\
\text { 2-Point }\end{array}$ \\
\hline
\end{tabular}

Tabelle 15: ICP-OES - Konzentrationen der Kalibrierlösungen

Kalibrierlösungen für die Hauptelemente $\mathrm{Ca}, \mathrm{Mg}, \mathrm{Na}$ (und $\mathrm{Al}^{*}$ ) [mg/l]

\begin{tabular}{|c|c|c|c|c|}
\hline $\mathbf{1}$ & $\mathbf{2}$ & $\mathbf{3}$ & $\mathbf{4}$ & $\mathbf{5}$ \\
\hline 0,1 & 1 & 10 & 100 & 500 \\
\hline
\end{tabular}

Kalibrierlösungen für das Hauptelement $\mathrm{P}\left(\right.$ als $\left.\mathrm{PO}_{4}\right)[\mu \mathrm{g} / \mathrm{ml}]$

\begin{tabular}{|c|c|c|c|c|c|}
\hline 1 & 2 & 3 & & & \\
\hline 10 & 100 & 1000 & & & \\
\hline \multicolumn{6}{|c|}{ Kalibrierlösungen für die Spurenelemente $\mathrm{Al}^{*}, \mathrm{Ba}$ und $\mathrm{Sr}[\mu \mathrm{g} / \mathrm{ml}]$} \\
\hline 1 & 2 & 3 & 4 & 5 & 6 \\
\hline 1 & 5 & 50 & 100 & 1000 & 5000 \\
\hline
\end{tabular}

* Al wurde in 2 überlappenden Konzentrationsbereichen kalibriert 


\subsubsection{EPMA}

Tabelle 16: EPMA - Analyseparameter

\begin{tabular}{|c|c|c|}
\hline Paramter & Einzelpunktanalysen & $\begin{array}{c}\text { Analyse für } \\
\text { Elementverteilungsbilder }\end{array}$ \\
\hline $\begin{array}{l}\text { Beschleunigungsspannung } \\
\text { Strahlstrom } \\
\text { Strahlfokussierung } \\
\text { Strahl auf Probe }(\varnothing)\end{array}$ & $\begin{array}{ll}15 \quad \mathrm{kV} \\
12 \mu \mathrm{nA} \\
\text { defokussiert } \\
5 \quad \mu \mathrm{m}\end{array}$ & $\begin{array}{cc}20 & \mathrm{kV} \\
40 & \mathrm{nA} \\
\text { maximale } & \text { Fokussierung }\end{array}$ \\
\hline
\end{tabular}

Tabelle 17: EPMA - Kalibrierstandards und elementspezifische Analyseparameter

\begin{tabular}{|c|c|c|c|c|c|c|}
\hline \multirow[t]{2}{*}{ Element } & \multicolumn{3}{|c|}{ Kalibrierstandards } & \multicolumn{2}{|c|}{$\begin{array}{l}\text { Einzelpunktanalysen } \\
\text { (WDS) }\end{array}$} & \multirow{2}{*}{\begin{tabular}{|c}
$\begin{array}{c}\text { Analysen zur } \\
\text { Element- } \\
\text { verteilung (WDS) }\end{array}$ \\
Meßzeit \\
(time per step) \\
{$[\mathrm{ms}]$}
\end{tabular}} \\
\hline & $\begin{array}{l}\text { Standard } \\
\text { Name }\end{array}$ & $\begin{array}{l}\text { Referenz } \\
\text {-oxid } \\
\text {-element }\end{array}$ & $\begin{array}{c}\text { Masseanteil } \\
\text { im Standard } \\
{[\%]}\end{array}$ & $\begin{array}{c}\text { Meßzeit } \\
\text { [sec] }\end{array}$ & $\begin{array}{c}\text { Meßzeit } \\
\text { Background } \\
{[\mathrm{sec}]}\end{array}$ & \\
\hline $\begin{array}{l}\mathrm{Ca} \\
\mathrm{P} \\
\mathrm{Mg} \\
\mathrm{Na} \\
\mathrm{S} \\
\mathrm{Sr} \\
\mathrm{Al} \\
\mathrm{Mn} \\
\mathrm{K} \\
\mathrm{F} \\
\mathrm{Cl} \\
\mathrm{Fe} \\
\mathrm{Si}\end{array}$ & 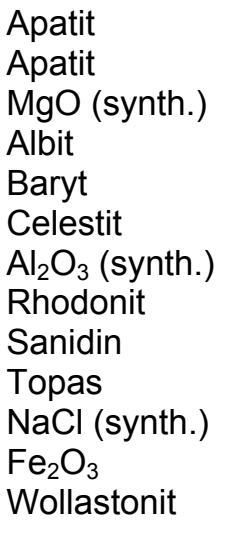 & $\begin{array}{l}\mathrm{CaO} \\
\mathrm{P}_{2} \mathrm{O}_{5} \\
\mathrm{MgO} \\
\mathrm{Na}_{2} \mathrm{O} \\
\mathrm{SO}_{3} \\
\mathrm{SrO} \\
\mathrm{Al}_{2} \mathrm{O}_{3} \\
\mathrm{MnO} \\
\mathrm{K}_{2} \mathrm{O} \\
\mathrm{F} \\
\mathrm{Cl} \\
\mathrm{FeO} \\
\mathrm{SiO}_{2}\end{array}$ & $\begin{array}{l}55,60 \\
42,23 \\
99,99 \\
11,74 \\
34,31 \\
56,41 \\
99,99 \\
42,44 \\
14,18 \\
20,46 \\
60,66 \\
89,71 \\
51,51\end{array}$ & $\begin{array}{l}15 \\
15 \\
15 \\
30 \\
30 \\
30 \\
30 \\
30 \\
15 \\
60 \\
30 \\
30 \\
15\end{array}$ & $\begin{array}{r}5 \\
5 \\
5 \\
15 \\
15 \\
15 \\
15 \\
15 \\
5 \\
30 \\
15 \\
15 \\
5\end{array}$ & $\begin{array}{c}80 \\
80 \\
80 \\
80 \\
\text { nicht gemessen } \\
\text { nicht gemessen } \\
\text { nicht gemessen } \\
\text { nicht gemessen } \\
\text { nicht gemessen } \\
\text { nicht gemessen } \\
80 \\
\text { nicht gemessen } \\
80 \text { (EDS) }\end{array}$ \\
\hline $\begin{array}{l}\text { WDS }= \\
\text { EDS }=\end{array}$ & $\begin{array}{l}\text { nalyse } \mathrm{m} \\
\text { nalyse } \mathrm{m}\end{array}$ & ה & . & $r$ & & \\
\hline
\end{tabular}




\subsubsection{LA-ICP-TOFMS}

Tabelle 18: LA-ICP-TOFMS - Geräte- und Analyseparameter (Angaben geräteseitig in englischer Sprache)

\begin{tabular}{|c|c|c|c|}
\hline Laser & $\begin{array}{l}\text { Type } \\
\text { Wavelengt } \\
\text { Energy } \\
\text { beam repetition rate } \\
\text { Ablation duration } \\
\text { pit size }\end{array}$ & $\begin{array}{l}\text { Excimer } \\
193 \\
125 \\
5 \\
2 \\
120\end{array}$ & $\begin{array}{l}(\mathrm{ArF}) \\
\mathrm{nm} \\
\mathrm{mJ} \\
\mathrm{Hz} \\
\mathrm{s} \\
\mu \mathrm{m}\end{array}$ \\
\hline ICP-TOFMS & $\begin{array}{l}\text { Forwarded power } \\
\text { Reflected power } \\
\text { Coolant gas flow (Ar) } \\
\text { Auxilary gas flow } \\
\text { Sample uptake rate } \\
\text { Skimmer type } \\
\text { Cone type } \\
\text { Expansion pressure } \\
\text { Intermediate pressure } \\
\text { Analyser pressure } \\
\text { Scanned mass range } \\
\text { Signal analysis } \\
\text { Integration time }\end{array}$ & $\begin{array}{ll} & 1,35 \\
< & 2 \\
15 \\
1 \\
& 0,97 \\
\mathrm{Ni} \quad & 0,8 \\
\mathrm{Ni} \quad & 0,9 \\
& 2,2 \\
< & 1 \times 10^{-4} \\
& 4 \times 10^{-6} \\
& { }^{6} \mathrm{Li}-{ }^{238} \mathrm{I} \\
& \text { transier } \\
255 & \end{array}$ & $\begin{array}{l}\mathrm{kW} \\
\mathrm{W} \\
\mathrm{I} / \mathrm{min} \\
\mathrm{I} / \mathrm{min} \\
\mathrm{I} / \mathrm{min} \\
\mathrm{mm} \\
\mathrm{mm} \\
\mathrm{mbar} \\
\mathrm{mbar} \\
\mathrm{mbar} \\
\mathrm{J} \\
\mathrm{ms}\end{array}$ \\
\hline
\end{tabular}

8.4

Konzentrationsberechnungen, Referenzmessungen, Meßfehler und Nachweisgrenzen

Tabelle 19: EPMA und ICP-OES - Faktoren für die Konzentrationsumrechnung Elementverbindung-Element

\begin{tabular}{|c|c|c|c|c|}
\hline \multirow{2}{*}{$\begin{array}{l}\text { Element } \\
\text { (Analyt) }\end{array}$} & \multirow{2}{*}{$\begin{array}{c}\text { Referenzverbindung } \\
\text { (Stöchiometrie) }\end{array}$} & \multicolumn{2}{|c|}{ relative Atommasse } & \multirow{2}{*}{$\begin{array}{c}\text { resultierender } \\
\text { Umrechnungsfaktor } \\
\text { (-divisor) }\end{array}$} \\
\hline & & Sauerstoff & Analyt & \\
\hline $\begin{array}{l}\mathrm{Ca} \\
\mathrm{P} \\
\mathrm{Mg} \\
\mathrm{Na} \\
\mathrm{S} \\
\mathrm{P}^{*}\end{array}$ & $\begin{array}{l}\mathrm{CaO} \\
\mathrm{P}_{2} \mathrm{O}_{5} \\
\mathrm{MgO} \\
\mathrm{Na}_{2} \mathrm{O} \\
\mathrm{SO}_{3} \\
\mathrm{PO}_{4}{ }^{*}\end{array}$ & $\begin{array}{l}15,999 \\
15,999 \\
15,999 \\
15,999 \\
15,999 \\
15,999^{\star}\end{array}$ & $\begin{array}{l}40,078 \\
30,974 \\
24,305 \\
22,990 \\
32,066 \\
30,974^{*}\end{array}$ & $\begin{array}{l}1,399 \\
2,291 \\
1,658 \\
1,348 \\
2,497 \\
3,066^{\star}\end{array}$ \\
\hline
\end{tabular}

* Berechnungsgrunlage für Phosphorbestimmung mit ICP-OES 
Tabelle 20: Konzentrationen der Referenzmaterialien SRM 1400 und Animal Bone H5

\begin{tabular}{|c|c|c|c|c|}
\hline \multirow[b]{2}{*}{ Element } & \multicolumn{2}{|c|}{ SRM 1400} & \multicolumn{2}{|c|}{ Animal Bone H5 } \\
\hline & Mittelwert & $\begin{array}{c}\text { Konfidenzintervall } \\
(95 \%)\end{array}$ & Mittelwert & $\begin{array}{c}\text { Konfidenzintervall } \\
(95 \%)\end{array}$ \\
\hline $\begin{array}{ll}\text { Ca } & {[\mathrm{mg} / \mathrm{g}]} \\
\mathbf{P} & {[\mathrm{mg} / \mathrm{g}]} \\
\mathrm{Sr} & {[\mu \mathrm{g} / \mathrm{g}]} \\
\mathrm{Ba} & {[\mu \mathrm{g} / \mathrm{g}]} \\
\mathrm{Mg} & {[\mu \mathrm{g} / \mathrm{g}]} \\
\mathrm{Al} & {[\mu \mathrm{g} / \mathrm{g}]} \\
\mathrm{Na} & {[\mu \mathrm{g} / \mathrm{g}]}\end{array}$ & $\begin{array}{c}381,8 \\
179,1 \\
249 \\
\text { keine Angaben } \\
6840 \\
530^{\star} \\
6000\end{array}$ & $\begin{array}{c}380,5-\quad 383,1 \\
177,2-\quad 181 \\
242 \quad-\quad 256 \\
\text { keine Angaben } \\
6710 \quad-\quad 6970 \\
\text { keine Angaben } \\
\text { keine Angaben }\end{array}$ & $\begin{array}{c}212 \\
102 \\
96 \\
79 \\
3550 \\
\text { keine Angaben } \\
5000\end{array}$ & $\begin{array}{rrr}204 & - & 220 \\
93 & - & 110 \\
88 & - & 105 \\
67 & - & 92 \\
3460 & - & 3640 \\
\text { keine } & \text { Angaben } \\
4700 & - & 5300\end{array}$ \\
\hline
\end{tabular}

Tabelle 21: FAAS - Ergebnisse der Referenzmessungen und resultierende relative Fehler für die Konzentrationsquotienten

\begin{tabular}{|c|c|c|c|c|c|c|}
\hline $\begin{array}{l}\text { Skelett- } \\
\text { serie }\end{array}$ & Element & $\begin{array}{c}\text { Referenz- } \\
\text { material }\end{array}$ & Mittelwert & \begin{tabular}{|c|} 
relative \\
Standard- \\
abweichung \\
{$[\%]$}
\end{tabular} & $\begin{array}{c}\text { Konzentrations- } \\
\text { quotient }\end{array}$ & $\begin{array}{c}\text { Gesamtfehler } \\
\text { Quotient } \\
{[\%]}\end{array}$ \\
\hline \multirow[t]{2}{*}{ Weingarten } & $\begin{array}{l}\text { Ca }[\mathrm{mg} / \mathrm{g}] \\
\text { P }[\mathrm{mg} / \mathrm{g}] \\
\text { Sr }[\mu \mathrm{g} / \mathrm{g}]\end{array}$ & $\begin{array}{l}\text { SRM 1400 } \\
\text { SRM 1400 } \\
\text { SRM 1400 }\end{array}$ & $\begin{array}{l}381 \\
181 \\
254\end{array}$ & $\begin{array}{l}0,9 \\
0,4 \\
1,2\end{array}$ & $\begin{array}{l}\mathrm{Ca} / \mathrm{P} \\
\mathrm{Sr} / \mathrm{Ca}\end{array}$ & $\begin{array}{l}0,9 \\
1,5\end{array}$ \\
\hline & $\begin{array}{l}\text { Ca }[\mathrm{mg} / \mathrm{g}] \\
\mathbf{P}[\mathrm{mg} / \mathrm{g}] \\
\mathrm{Ba}[\mu \mathrm{g} / \mathrm{g}] \\
\text { Sr }[\mu \mathrm{g} / \mathrm{g}]\end{array}$ & $\begin{array}{l}\mathrm{H} 5 \\
\mathrm{H} 5 \\
\mathrm{H} 5 \\
\mathrm{H} 5\end{array}$ & $\begin{array}{r}213 \\
103 \\
79 \\
100\end{array}$ & $\begin{array}{r}2,3 \\
2,0 \\
14,8 \\
5,1\end{array}$ & $\begin{array}{l}\mathrm{Ca} / \mathrm{P} \\
\mathrm{Ba} / \mathrm{Ca} \\
\mathrm{Sr} / \mathrm{Ca}\end{array}$ & $\begin{array}{r}3,1 \\
14,9 \\
5,6\end{array}$ \\
\hline $\begin{array}{l}\text { Kircheim } \\
\text { unter Teck }\end{array}$ & $\begin{array}{l}\text { Ca }[\mathrm{mg} / \mathrm{g}] \\
\mathbf{P}[\mathrm{mg} / \mathrm{g}] \\
\mathrm{Ba}[\mu \mathrm{g} / \mathrm{g}] \\
\text { Sr }[\mu \mathrm{g} / \mathrm{g}]\end{array}$ & $\begin{array}{l}\mathrm{H} 5 \\
\mathrm{H} 5 \\
\mathrm{H} 5 \\
\mathrm{H} 5\end{array}$ & $\begin{array}{r}212 \\
102 \\
84 \\
103\end{array}$ & $\begin{array}{l}0,7 \\
1,1 \\
3,2 \\
2,5\end{array}$ & $\begin{array}{l}\mathrm{Ca} / \mathrm{P} \\
\mathrm{Ba} / \mathrm{Ca} \\
\mathrm{Sr} / \mathrm{Ca}\end{array}$ & $\begin{array}{l}1,3 \\
3,2 \\
2,6\end{array}$ \\
\hline Wenigumstadt & $\begin{array}{l}\mathrm{Ca}[\mathrm{mg} / \mathrm{g}] \\
\mathrm{P}[\mathrm{mg} / \mathrm{g}] \\
\mathrm{Ba}[\mu \mathrm{g} / \mathrm{g}] \\
\mathrm{Sr}[\mu \mathrm{g} / \mathrm{g}]\end{array}$ & $\mid \begin{array}{c}\text { SRM } 1400 \\
\text { SRM } 1400 \\
\text { H } 5 \\
\text { SRM } 1400\end{array}$ & $\begin{array}{r}382 \\
179 \\
82 \\
255\end{array}$ & $\begin{array}{l}1 \\
1,3 \\
7,4 \\
1,5\end{array}$ & $\begin{array}{l}\text { Ca/P } \\
\text { Ba/Ca } \\
\text { Sr/Ca }\end{array}$ & $\begin{array}{l}1,7 \\
7,4 \\
1,8\end{array}$ \\
\hline
\end{tabular}


Tabelle 22: ICP-OES - Ergebnisse der Referenzmessungen des SRM 1400 und die resultierenden relativen Fehler für die Konzentrationsquotienten

\begin{tabular}{|c|c|c|c|c|}
\hline Element & $\begin{array}{c}\text { Mittelwert } \\
(n=62)\end{array}$ & \begin{tabular}{|c|} 
relative \\
Standard- \\
abweichung \\
{$[\%]$}
\end{tabular} & $\begin{array}{c}\text { Konzentrations- } \\
\text { quotient }\end{array}$ & $\begin{array}{c}\text { Gesamtfehler } \\
\text { Quotient } \\
\text { [\%] }\end{array}$ \\
\hline Ca $[\mathrm{mg} / \mathrm{g}]$ & 374 & 1,0 & & \\
\hline P $\quad[\mathrm{mg} / \mathrm{g}]$ & 176 & 1,1 & $\mathrm{Ca} / \mathrm{P}$ & 1,5 \\
\hline $\mathrm{Ba}[\mu \mathrm{g} / \mathrm{g}]$ & 222 & 0,9 & $\mathrm{Ba} / \mathrm{Ca}$ & 1,4 \\
\hline $\mathrm{Sr} \quad[\mu \mathrm{g} / \mathrm{g}]$ & 225 & 1,0 & $\mathrm{Sr} / \mathrm{Ca}$ & 1,4 \\
\hline $\mathrm{Mg}[\mu \mathrm{g} / \mathrm{q}]$ & 7236 & 0,9 & $\mathrm{Mg} / \mathrm{Ca}$ & 1,4 \\
\hline Al $[\mu \mathrm{g} / \mathrm{g}]$ & 380 & 1,5 & $\mathrm{Al} / \mathrm{Ca}$ & 1,9 \\
\hline $\mathrm{Na} \quad[\mu \mathrm{g} / \mathrm{g}]$ & $7723(n=6)$ & 0,7 & $\mathrm{Na} / \mathrm{Ca}$ & 1,2 \\
\hline
\end{tabular}

$\mathrm{n}=$ Anzahl der Referenzmessungen

Tabelle 23: ICP-OES - Nachweisgrenzen für die Elemente in der Meßlösung und deren Umrechnung auf Nachweisgrenze als Knochenkonzentrationen

\begin{tabular}{|l|l|l|}
\hline Element & $\begin{array}{l}\text { Nachweisgrenze } \\
\text { in } \\
\text { Meßlösung } \\
{[\mu \mathrm{g} / \mathrm{ml}]}\end{array}$ & $\begin{array}{l}\text { Nachweisgrenze } \\
\text { für } \\
\text { Knochensubstanz } \\
{[\mu \mathrm{g} / \mathrm{g}]}\end{array}$ \\
\hline $\mathrm{Ca}$ & 0,05 & 50 \\
$\mathbf{P}\left(\right.$ als $\left.\mathrm{PO}_{4}\right)$ & 0,02 & $10 \quad$ (auf P umgerechnet, vgl. Tabelle 19) \\
$\mathbf{B a}$ & 0,005 & 5 \\
$\mathbf{S r}$ & 0,005 & 5 \\
$\mathbf{M g}$ & 0,05 & 50 \\
$\mathbf{A l}$ & 0,01 & 10 \\
$\mathbf{N a}$ & 0,1 & 100 \\
\hline
\end{tabular}

* umgerechnet und auf $10 \mu \mathrm{g}$ gerundet mit Verdünnung 1:7, Stammlösungsvolumen von $10 \mathrm{ml}$ und mittlerer Knocheneinwaage von $0,070 \mathrm{~g}$

Tabelle 24: EPMA - Nachweisgrenzen und relative Fehler der auswertungsrelevanten Elemente einschließlich der resultierenden relativen Fehler für die Konzentrationsquotienten.

\begin{tabular}{|c|c|c|c|c|c|}
\hline Element & $\begin{array}{c}\text { Gemessene } \\
\text { Konzentration* }\end{array}$ & $\begin{array}{c}\text { Nachweis- } \\
\text { grenze } \\
{[\mu \mathrm{g} / \mathrm{g}]}\end{array}$ & $\begin{array}{c}\text { relative } \\
\text { Standard- } \\
\text { abweichung } \\
{[\%]}\end{array}$ & $\begin{array}{c}\text { Konzentrations- } \\
\text { quotient }\end{array}$ & $\begin{array}{c}\text { Gesamtfehler } \\
\text { Quotient } \\
{[\%]}\end{array}$ \\
\hline $\begin{array}{l}\mathrm{Ca} \\
\mathrm{P} \\
\mathrm{Mg} \\
\mathrm{Na} \\
\mathrm{S}\end{array}$ & $\begin{aligned} 294,5 & {[\mathrm{mg} / \mathrm{g}] } \\
128,6 & {[\mathrm{mg} / \mathrm{g}] } \\
764 & {[\mu \mathrm{g} / \mathrm{g}] } \\
2255 & {[\mu \mathrm{g} / \mathrm{g}] } \\
1106 & {[\mu \mathrm{g} / \mathrm{g}] }\end{aligned}$ & $\begin{array}{r}400 \\
1500 \\
190 \\
350 \\
220\end{array}$ & $\begin{array}{r}0,5 \\
1,2 \\
13,1 \\
7,1 \\
8,8\end{array}$ & $\begin{array}{l}\mathrm{Ca} / \mathrm{P} \\
\mathrm{Mg} / \mathrm{Ca} \\
\mathrm{Na} / \mathrm{Ca} \\
\mathrm{S} / \mathrm{Ca}\end{array}$ & $\begin{array}{r}1,3 \\
13,1 \\
7,1 \\
8,8\end{array}$ \\
\hline
\end{tabular}

${ }^{*}$ Meßergebnisse einer Einzelpunktmessung mit typischen (mittleren) Konzentrationen. Dieser Meßpunkt bildete die Grundlage für die Fehlerschätzungen. 


\subsection{Probenlisten, Meßdaten und tabellierte statistisch deskriptive Daten}

Tabelle 25: Weingarten - Probenliste und auswertungsrelevante Individualdaten.

\begin{tabular}{|c|c|c|c|c|c|c|c|c|c|c|c|c|}
\hline \multirow[t]{2}{*}{ Grab } & \multirow{2}{*}{$\begin{array}{l}\text { Sex } \\
\mathrm{m} / \mathrm{w}\end{array}$} & \multirow{2}{*}{$\begin{array}{c}\text { Rang } \\
0 / 1\end{array}$} & \multirow[t]{2}{*}{ Histologie } & \multicolumn{2}{|c|}{ Masseverlust [\%] } & \multirow{2}{*}{$\begin{array}{c}\mathrm{Ca} \\
{[\mathrm{mg} / \mathrm{g}]}\end{array}$} & \multirow{2}{*}{$\begin{array}{c}P \\
{[\mathrm{mg} / \mathrm{g}]}\end{array}$} & \multirow{2}{*}{$\begin{array}{c}\mathrm{Ba} \\
{[\mu \mathrm{g} / \mathrm{g}]}\end{array}$} & \multirow{2}{*}{$\begin{array}{c}\mathrm{Sr} \\
{[\mu \mathrm{g} / \mathrm{g}]}\end{array}$} & $\mathrm{Ca} / \mathrm{P}$ & $\mathrm{Ba} / \mathrm{Ca}$ & $\mathrm{Sr} / \mathrm{Ca}$ \\
\hline & & & & Ätzen & Veraschen & & & & & & {$\left[\times 10^{-3}\right]$} & {$\left[\times 10^{-3}\right]$} \\
\hline 63 & $\mathrm{~m}$ & 0 & schlecht & 16,8 & 18,8 & 380 & & 132 & 519 & & 0,35 & 1,37 \\
\hline 66 & w & 1 & gut & 8,1 & 29,5 & 375 & 165 & 101 & 364 & 2,27 & 0,27 & 0,97 \\
\hline 79 & w & 0 & schlecht & 12,6 & 16,4 & 377 & 169 & 119 & 492 & 2,24 & 0,32 & 1,31 \\
\hline 111 & w & 1 & schlecht & 15,6 & 22,4 & 379 & & 137 & 585 & & 0,36 & 1,54 \\
\hline 113 & w & 1 & gut & & & 369 & 175 & 87 & 266 & 2,11 & 0,23 & 0,72 \\
\hline 115 & $\mathrm{~m}$ & 0 & schlecht & & & 363 & 173 & 129 & 650 & 2,10 & 0,36 & 1,79 \\
\hline 118 & w & 1 & schlecht & 9,0 & 16,3 & 365 & 172 & 100 & 404 & 2,12 & 0,27 & 1,11 \\
\hline 119 & w & 1 & gut & & & 383 & 172 & 59 & 340 & 2,22 & 0,16 & 0,89 \\
\hline 130 & w & 0 & schlecht & 20,7 & & 369 & 175 & 85 & 474 & 2,12 & 0,23 & 1,28 \\
\hline 134 & $\mathrm{~m}$ & 0 & gut & & & 384 & 169 & 49 & 301 & 2,28 & 0,13 & 0,78 \\
\hline 136 & $\mathrm{~m}$ & 1 & gut & 12,6 & 29,0 & 369 & 163 & 79 & 305 & 2,26 & 0,21 & 0,82 \\
\hline 141 & w & 0 & schlecht & 9,5 & 28,2 & 373 & & 71 & 272 & & 0,19 & 0,73 \\
\hline 144 & $\mathrm{~m}$ & 0 & schlecht & & & 362 & 172 & 108 & 594 & 2,10 & 0,30 & 1,64 \\
\hline 153 & w & 1 & schlecht & 20,5 & 13,3 & 346 & 175 & 79 & 440 & 1,98 & 0,23 & 1,27 \\
\hline 157 & w & 0 & schlecht & & & 350 & 171 & 85 & 412 & 2,04 & 0,24 & 1,18 \\
\hline 160 & $\mathrm{~m}$ & 0 & schlecht & & & 353 & 165 & 138 & 518 & 2,14 & 0,39 & 1,47 \\
\hline 161 & w & 1 & gut & 12,0 & 29,6 & 370 & & 103 & 253 & & 0,28 & 0,68 \\
\hline 163 & $\mathrm{~m}$ & 0 & gut & & & 350 & 170 & 80 & 316 & 2,06 & 0,23 & 0,90 \\
\hline 165 & $\mathrm{~m}$ & 1 & schlecht & 17,6 & 19,8 & 370 & & 129 & 581 & & 0,35 & 1,57 \\
\hline 166 & w & 0 & schlecht & & & 356 & 180 & 107 & 623 & 1,98 & 0,30 & 1,75 \\
\hline 167 & $\mathrm{~m}$ & 1 & schlecht & & & 362 & 180 & 92 & 479 & 2,01 & 0,25 & 1,32 \\
\hline 171 & $\mathrm{~m}$ & 0 & schlecht & & & 429 & 179 & 117 & 572 & 2,40 & 0,27 & 1,33 \\
\hline 175 & w & 0 & gut & & & 358 & 162 & 63 & 255 & 2,21 & 0,18 & 0,71 \\
\hline 178 & w & 1 & gut & 8,2 & 28,2 & 373 & & 59 & 272 & & 0,16 & 0,73 \\
\hline 179 & w & 1 & schlecht & 11,1 & 21,0 & 379 & & 71 & 530 & & 0,19 & 1,40 \\
\hline 180 & w & 0 & schlecht & 11,7 & & 397 & 174 & 106 & 561 & 2,28 & 0,27 & 1,41 \\
\hline 182 & $\mathrm{~m}$ & 0 & schlecht & & & 351 & 179 & 109 & 502 & 1,96 & 0,31 & 1,43 \\
\hline 184 & w & 0 & schlecht & & & 345 & 170 & 131 & 548 & 2,03 & 0,38 & 1,59 \\
\hline 186 & $\mathrm{~m}$ & 0 & schlecht & & & 409 & 175 & 129 & 608 & 2,34 & 0,32 & 1,49 \\
\hline 187 & w & 1 & schlecht & & & 402 & 165 & 110 & 599 & 2,44 & 0,27 & 1,49 \\
\hline 194 & w & 1 & schlecht & & & 392 & 174 & 98 & 491 & 2,26 & 0,25 & 1,25 \\
\hline 196 & w & 0 & schlecht & 25,2 & & 370 & 179 & 129 & 530 & 2,07 & 0,35 & 1,43 \\
\hline 201 & $\mathrm{~m}$ & 1 & gut & & & 354 & 166 & 83 & 255 & 2,13 & 0,23 & 0,72 \\
\hline 202 & $\mathrm{~m}$ & 1 & gut & & & 386 & 164 & 83 & 315 & 2,36 & 0,22 & 0,82 \\
\hline 206 & w & 0 & gut & & & 351 & 170 & 93 & 378 & 2,07 & 0,27 & 1,08 \\
\hline 208 & $\mathrm{~m}$ & 0 & gut & & & 375 & 162 & 90 & 293 & 2,32 & 0,24 & 0,78 \\
\hline 211 & $\mathrm{~m}$ & 0 & schlecht & & & 379 & 165 & 67 & 549 & 2,29 & 0,18 & 1,45 \\
\hline 220 & w & 1 & schlecht & 6,2 & 31,6 & 376 & & 68 & 351 & & 0,18 & 0,93 \\
\hline 221 & w & 0 & schlecht & 22,8 & & 341 & 181 & 110 & 578 & 1,88 & 0,32 & 1,69 \\
\hline 227 & $\mathrm{~m}$ & 1 & gut & & & 374 & 159 & 44 & 342 & 2,35 & 0,12 & 0,91 \\
\hline 228 & w & 0 & schlecht & & & 384 & 173 & 107 & 505 & 2,22 & 0,28 & 1,31 \\
\hline 231 & $\mathrm{~m}$ & 0 & schlecht & & & 356 & 178 & 101 & 582 & 2,00 & 0,29 & 1,64 \\
\hline 234 & w & 0 & schlecht & & & 380 & 164 & 104 & 566 & 2,33 & 0,27 & 1,49 \\
\hline 241 & w & 1 & gut & 11,8 & 28,9 & 370 & & 69 & 310 & & 0,19 & 0,84 \\
\hline 253 & $\mathrm{~m}$ & 1 & gut & 11,6 & 29,4 & 380 & & 91 & 308 & & 0,24 & 0,81 \\
\hline 256 & $\mathrm{~m}$ & 1 & gut & & & 345 & 161 & 82 & 316 & 2,15 & 0,24 & 0,91 \\
\hline 257 & $\mathrm{~m}$ & 1 & schlecht & & & 371 & 166 & 131 & 562 & 2,24 & 0,35 & 1,52 \\
\hline 258 & w & 0 & schlecht & & & 353 & 169 & 119 & 621 & 2,09 & 0,34 & 1,76 \\
\hline 259 & $\mathrm{~m}$ & 0 & schlecht & & & 372 & 166 & 107 & 464 & 2,25 & 0,29 & 1,25 \\
\hline 270 & $\mathrm{~m}$ & 0 & schlecht & & & 364 & 166 & 123 & 452 & 2,20 & 0,34 & 1,24 \\
\hline 271 & w & 1 & schlecht & & & 326 & 174 & 131 & 584 & 1,87 & 0,40 & 1,79 \\
\hline 272 & w & 1 & schlecht & & & 369 & 177 & 119 & 556 & 2,08 & 0,32 & 1,51 \\
\hline 275 & $\mathrm{~m}$ & 1 & gut & 9,1 & 28,8 & 383 & & 110 & 372 & & 0,29 & 0,97 \\
\hline 278 & w & 0 & schlecht & & & 349 & 172 & 132 & 556 & 2,03 & 0,38 & 1,59 \\
\hline 283 & $\mathrm{~m}$ & 1 & gut & & & 370 & 158 & 88 & 302 & 2,34 & 0,24 & 0,82 \\
\hline 293 & w & 0 & schlecht & & & 356 & 174 & 96 & 509 & 2,05 & 0,27 & 1,43 \\
\hline 298 & $\mathrm{~m}$ & 0 & schlecht & & & 363 & 170 & 130 & 411 & 2,14 & 0,36 & 1,13 \\
\hline 303 & $\mathrm{~m}$ & 0 & schlecht & & & 377 & 164 & 161 & 593 & 2,30 & 0,43 & 1,57 \\
\hline 313 & w & 1 & gut & 10,9 & 27,6 & 380 & & 107 & 275 & & 0,28 & 0,73 \\
\hline 322 & $\mathrm{~m}$ & 0 & schlecht & & & 377 & 169 & 150 & 465 & 2,23 & 0,40 & 1,23 \\
\hline 323 & $\mathrm{~m}$ & 1 & gut & & & 354 & 164 & 151 & 311 & 2,16 & 0,43 & 0,88 \\
\hline 326 & $\mathrm{~m}$ & 0 & gut & & & 363 & 160 & 107 & 284 & 2,27 & 0,29 & 0,78 \\
\hline 329 & $\mathrm{~m}$ & 0 & schlecht & & & 369 & 170 & 126 & 465 & 2,17 & 0,34 & 1,26 \\
\hline 330 & $\mathrm{~m}$ & 1 & gut & & & 341 & 162 & 102 & 283 & 2,10 & 0,30 & 0,83 \\
\hline 332 & $\mathrm{~m}$ & 1 & schlecht & 11,4 & 31,9 & 384 & & 113 & 419 & & 0,29 & 1,09 \\
\hline 335 & $\mathrm{~m}$ & 1 & schlecht & & & 355 & 166 & 125 & 649 & 2,14 & 0,35 & 1,83 \\
\hline
\end{tabular}


Fortsetzung Tabelle 25

\begin{tabular}{|c|c|c|c|c|c|c|c|c|c|c|c|c|}
\hline \multirow[t]{2}{*}{ Grab } & \multirow{2}{*}{$\begin{array}{l}\text { Sex } \\
m / w\end{array}$} & \multirow{2}{*}{$\begin{array}{c}\text { Rang } \\
0 / 1\end{array}$} & \multirow[t]{2}{*}{ Histologie } & \multicolumn{2}{|c|}{ Masseverlust [\%] } & \multirow{2}{*}{$\begin{array}{c}\mathrm{Ca} \\
{[\mathrm{mg} / \mathrm{g}]}\end{array}$} & $\mathbf{P}$ & $\mathrm{Ba}$ & $\mathrm{Sr}$ & $\mathrm{Ca} / \mathrm{P}$ & $\mathrm{Ba} / \mathrm{Ca}$ & $\mathrm{Sr} / \mathrm{Ca}$ \\
\hline & & & & Ätzen & Veraschen & & {$[\mathrm{mg} / \mathrm{g}]$} & {$[\mu \mathrm{g} / \mathrm{g}]$} & {$[\mu \mathrm{g} / \mathrm{g}]$} & & {$\left[\times 10^{-3}\right]$} & {$\left[\times 10^{-3}\right]$} \\
\hline 342 & $\mathrm{~m}$ & 1 & gut & 5,6 & 31,8 & 379 & & 87 & 353 & & 0,23 & 0,93 \\
\hline 343 & $\mathrm{~m}$ & 0 & schlecht & 24,0 & 11,7 & 367 & & 120 & 549 & & 0,33 & 1,50 \\
\hline 344 & w & 1 & gut & & & 345 & 162 & 115 & 343 & 2,13 & 0,33 & 1,00 \\
\hline 348 & w & 0 & schlecht & & & 347 & 164 & 138 & 565 & 2,12 & 0,40 & 1,63 \\
\hline 354 & w & 0 & schlecht & 18,3 & 18,1 & 375 & & 105 & 457 & & 0,28 & 1,22 \\
\hline 355 & w & 1 & schlecht & & & 365 & 168 & 101 & 512 & 2,17 & 0,28 & 1,40 \\
\hline 359 & $\mathrm{~m}$ & 1 & schlecht & & & 378 & 164 & 126 & 540 & 2,30 & 0,33 & 1,43 \\
\hline 360 & w & 1 & gut & 13,5 & 30,1 & 374 & & 92 & 329 & & 0,25 & 0,88 \\
\hline 363 & $\mathrm{~m}$ & 0 & schlecht & & & 353 & 170 & 101 & 468 & 2,08 & 0,29 & 1,33 \\
\hline 366 & $\mathrm{~m}$ & 0 & schlecht & & & 363 & 166 & 107 & 558 & 2,18 & 0,29 & 1,54 \\
\hline 367 & w & 0 & schlecht & 24,4 & 24,7 & 384 & 168 & 156 & 484 & 2,28 & 0,41 & 1,26 \\
\hline 370 & $\mathrm{~m}$ & 0 & schlecht & & & 355 & 165 & 136 & 476 & 2,15 & 0,38 & 1,34 \\
\hline 374 & w & 0 & gut & & & 359 & 164 & 36 & 324 & 2,19 & 0,10 & 0,90 \\
\hline 377 & $\mathrm{~m}$ & 1 & gut & 8,9 & 29,0 & 366 & & 89 & 264 & & 0,24 & 0,72 \\
\hline 379 & $\mathrm{~m}$ & 0 & schlecht & & & 355 & 162 & 110 & 492 & 2,19 & 0,31 & 1,39 \\
\hline 382 & w & 0 & gut & & & 354 & 165 & 92 & 281 & 2,14 & 0,26 & 0,79 \\
\hline 383 & w & 0 & schlecht & & & 358 & 164 & 119 & 529 & 2,19 & 0,33 & 1,48 \\
\hline 385 & $\mathrm{~m}$ & 1 & gut & 17,3 & 22,5 & 359 & 160 & 83 & 289 & 2,25 & 0,23 & 0,81 \\
\hline 395 & w & 1 & gut & 10,8 & 28,7 & 372 & & 87 & 297 & & 0,23 & 0,80 \\
\hline 396 & $\mathrm{~m}$ & 0 & schlecht & & & 378 & 166 & 128 & 527 & 2,28 & 0,34 & 1,39 \\
\hline 399 & $\mathrm{~m}$ & 1 & gut & & & 358 & 163 & 82 & 323 & 2,20 & 0,23 & 0,90 \\
\hline 405 & w & 0 & gut & 9,5 & 31,8 & 373 & & 93 & 293 & & 0,25 & 0,79 \\
\hline 408 & w & 0 & schlecht & & & 360 & 168 & 105 & 442 & 2,14 & 0,29 & 1,23 \\
\hline 409 & $\mathrm{~m}$ & 1 & gut & & & 373 & 161 & 82 & 337 & 2,31 & 0,22 & 0,90 \\
\hline 411 & w & 0 & gut & 8,6 & 30,5 & 373 & & 78 & 391 & & 0,21 & 1,05 \\
\hline 414 & w & 1 & schlecht & & & 394 & 180 & 99 & 487 & 2,19 & 0,25 & 1,23 \\
\hline 415 & $\mathrm{~m}$ & 0 & schlecht & 26,1 & 18,8 & 386 & & 104 & 576 & & 0,27 & 1,49 \\
\hline 416 & w & 1 & schlecht & 14,7 & & 377 & 166 & 98 & 565 & 2,27 & 0,26 & 1,50 \\
\hline 417 & w & 0 & schlecht & & & 397 & 163 & 113 & 558 & 2,43 & 0,29 & 1,41 \\
\hline 422 & w & 1 & schlecht & & & 348 & 169 & 139 & 524 & 2,05 & 0,40 & 1,51 \\
\hline 423 & $\mathrm{~m}$ & 1 & schlecht & & & 342 & 166 & 85 & 539 & 2,06 & 0,25 & 1,58 \\
\hline 427 & w & 0 & schlecht & 25,9 & & 347 & 173 & 117 & 533 & 2,00 & 0,34 & 1,54 \\
\hline 441 & w & 0 & schlecht & 29,9 & & 358 & 170 & 80 & 440 & 2,11 & 0,22 & 1,23 \\
\hline 442 & $\mathrm{~m}$ & 0 & schlecht & & & 363 & 170 & 102 & 444 & 2,14 & 0,28 & 1,22 \\
\hline 456 & w & 0 & schlecht & 16,8 & & 365 & 166 & 146 & 556 & 2,20 & 0,40 & 1,52 \\
\hline 464 & w & 1 & gut & 5,7 & & 352 & 158 & 82 & 295 & 2,23 & 0,23 & 0,84 \\
\hline 465 & $\mathrm{~m}$ & 1 & schlecht & 29,9 & 13,5 & 362 & & 141 & 501 & & 0,39 & 1,38 \\
\hline 469 & $\mathrm{~m}$ & 0 & schlecht & 21,8 & 17,6 & 389 & 166 & 123 & 547 & 2,35 & 0,32 & 1,41 \\
\hline 470 & w & 1 & gut & 10,8 & 29,0 & 378 & & 79 & 293 & & 0,21 & 0,78 \\
\hline 473 & w & 1 & gut & 8,1 & 31,2 & 378 & & 117 & 335 & & 0,31 & 0,89 \\
\hline 477 & w & 0 & schlecht & & & 374 & 165 & 110 & 489 & 2,27 & 0,29 & 1,31 \\
\hline 478 & $\mathrm{~m}$ & 1 & gut & & & 358 & 159 & 95 & 302 & 2,25 & 0,26 & 0,84 \\
\hline 480 & $\mathrm{~m}$ & 0 & schlecht & & & 370 & 161 & 116 & 428 & 2,29 & 0,31 & 1,16 \\
\hline 481 & w & 1 & schlecht & 14,6 & & 342 & 160 & 115 & 500 & 2,14 & 0,34 & 1,46 \\
\hline 482 & $\mathrm{~m}$ & 1 & gut & & & 371 & 175 & 75 & 244 & 2,13 & 0,20 & 0,66 \\
\hline 485 & $\mathrm{~m}$ & 0 & gut & & & 361 & 174 & 89 & 330 & 2,08 & 0,25 & 0,91 \\
\hline 487 & w & 1 & schlecht & 20,0 & 24,8 & 388 & & 113 & 535 & & 0,29 & 1,38 \\
\hline 488 & w & 0 & schlecht & & & 376 & 166 & 114 & 481 & 2,26 & 0,30 & 1,28 \\
\hline 494 & w & 0 & schlecht & 46,8 & 13,7 & 346 & 172 & 110 & 450 & 2,02 & 0,32 & 1,30 \\
\hline 496 & w & 1 & schlecht & & & 350 & 170 & 107 & 366 & 2,05 & 0,31 & 1,05 \\
\hline 497 & $\mathrm{~m}$ & 0 & gut & & & 390 & 173 & 87 & 509 & 2,25 & 0,22 & 1,30 \\
\hline 505 & w & 0 & schlecht & & & 371 & 169 & 151 & 530 & 2,19 & 0,41 & 1,43 \\
\hline 506 & $\mathrm{~m}$ & 0 & schlecht & & & 372 & 166 & 85 & 542 & 2,24 & 0,23 & 1,46 \\
\hline 507 & w & 1 & schlecht & & & 364 & 167 & 88 & 556 & 2,18 & 0,24 & 1,52 \\
\hline 511 & w & 1 & schlecht & 30,1 & 17,4 & 374 & & 123 & 494 & & 0,33 & 1,32 \\
\hline 556 & w & 1 & schlecht & & & 363 & 169 & 126 & 534 & 2,15 & 0,35 & 1,47 \\
\hline 560 & w & 0 & schlecht & & & 384 & 179 & 90 & 368 & 2,15 & 0,23 & 0,96 \\
\hline 562 & $\mathrm{~m}$ & 0 & schlecht & & & 354 & 168 & 88 & 495 & 2,11 & 0,25 & 1,40 \\
\hline 565 & $\mathrm{~m}$ & 1 & schlecht & & & 356 & 166 & 119 & 488 & 2,15 & 0,33 & 1,37 \\
\hline 567 & w & 1 & gut & & & 345 & 168 & 111 & 344 & 2,06 & 0,32 & 1,00 \\
\hline 568 & w & 0 & schlecht & & & 389 & 164 & 137 & 490 & 2,38 & 0,35 & 1,26 \\
\hline 569 & w & 0 & schlecht & & & 366 & 166 & 81 & 569 & 2,20 & 0,22 & 1,55 \\
\hline 576 & w & 0 & schlecht & 35,5 & 24,9 & 377 & & 107 & 356 & & 0,28 & 0,94 \\
\hline 577 & w & 1 & gut & 9,8 & 28,6 & 373 & & 95 & 321 & & 0,26 & 0,86 \\
\hline 580 & w & 1 & schlecht & & & 354 & 163 & 80 & 554 & 2,17 & 0,22 & 1,56 \\
\hline 586 & w & 0 & schlecht & & & 340 & 164 & 107 & 591 & 2,07 & 0,31 & 1,74 \\
\hline 587 & w & 0 & schlecht & & & 339 & 167 & 121 & 545 & 2,03 & 0,36 & 1,61 \\
\hline 600 & w & 1 & schlecht & 16,1 & & 338 & 173 & 112 & 570 & 1,96 & 0,33 & 1,69 \\
\hline 611 & w & 1 & schlecht & 18,4 & 21,2 & 380 & & 151 & 535 & & 0,40 & 1,41 \\
\hline 615 & w & 1 & gut & 10,3 & 28,9 & 384 & & 63 & 339 & & 0,16 & 0,88 \\
\hline 616 & $\mathrm{~m}$ & 1 & gut & 10,3 & 29,8 & 373 & & 105 & 298 & & 0,28 & 0,80 \\
\hline
\end{tabular}


Fortsetzung Tabelle 25

\begin{tabular}{|c|c|c|c|c|c|c|c|c|c|c|c|c|}
\hline \multirow[t]{2}{*}{ Grab } & \multirow{2}{*}{$\begin{array}{l}\text { Sex } \\
\mathrm{m} / \mathrm{w}\end{array}$} & \multirow{2}{*}{$\begin{array}{c}\text { Rang } \\
0 / 1\end{array}$} & \multirow[t]{2}{*}{ Histologie } & \multicolumn{2}{|c|}{ Masseverlust [\%] } & \multirow{2}{*}{$\begin{array}{c}\mathrm{Ca} \\
{[\mathrm{mg} / \mathrm{g}]}\end{array}$} & \multirow{2}{*}{$\begin{array}{c}P \\
{[\mathrm{mg} / \mathrm{g}]}\end{array}$} & \multirow{2}{*}{$\begin{array}{c}\mathrm{Ba} \\
{[\mu \mathrm{g} / \mathrm{g}]}\end{array}$} & \multirow{2}{*}{$\begin{array}{c}\mathrm{Sr} \\
{[\mu \mathrm{g} / \mathrm{g}]}\end{array}$} & \multirow[t]{2}{*}{$\mathrm{Ca} / \mathrm{P}$} & \multirow{2}{*}{$\begin{array}{l}\mathrm{Ba} / \mathrm{Ca} \\
{\left[\times 10^{-3}\right]}\end{array}$} & \multirow{2}{*}{$\begin{array}{l}\mathrm{Sr} / \mathrm{Ca} \\
{\left[\times 10^{-3}\right]}\end{array}$} \\
\hline & & & & Ätzen & Veraschen & & & & & & & \\
\hline 619 & $\mathrm{~m}$ & 1 & gut & 6,6 & 31,0 & 378 & & 70 & 332 & & 0,18 & 0,88 \\
\hline 620 & w & 1 & gut & 20,2 & 22,9 & 383 & & 101 & 614 & & 0,26 & 1,60 \\
\hline 625 & $\mathrm{~m}$ & 0 & schlecht & 18,2 & 17,2 & 377 & & 124 & 569 & & 0,33 & 1,51 \\
\hline 630 & w & 0 & schlecht & & & 335 & 164 & 116 & 516 & 2,05 & 0,35 & 1,54 \\
\hline 631 & $\mathrm{~m}$ & 0 & schlecht & & & 368 & 165 & 94 & 566 & 2,23 & 0,26 & 1,54 \\
\hline 636 & $\mathrm{~m}$ & 1 & schlecht & & & 378 & 164 & 103 & 552 & 2,30 & 0,27 & 1,46 \\
\hline 638 & w & 0 & schlecht & & & 363 & 169 & 72 & 445 & 2,14 & 0,20 & 1,23 \\
\hline 641 & $\mathrm{~m}$ & 0 & gut & & & 377 & 159 & 77 & 285 & 2,37 & 0,20 & 0,76 \\
\hline 644 & $\mathrm{~m}$ & 0 & schlecht & & & 368 & 166 & 81 & 369 & 2,21 & 0,22 & 1,00 \\
\hline 648 & w & 0 & schlecht & & & 371 & 166 & 87 & 419 & 2,24 & 0,23 & 1,13 \\
\hline 654 & $\mathrm{~m}$ & 1 & schlecht & & & 386 & 167 & 131 & 525 & 2,32 & 0,34 & 1,36 \\
\hline 656 & w & 0 & schlecht & & & 373 & 166 & 131 & 535 & 2,24 & 0,35 & 1,44 \\
\hline 658 & w & 0 & schlecht & & & 364 & 170 & 108 & 524 & 2,14 & 0,30 & 1,44 \\
\hline 664 & w & 0 & schlecht & & & 359 & 164 & 122 & 525 & 2,19 & 0,34 & 1,46 \\
\hline 668 & w & 1 & schlecht & 19,6 & 22,6 & 382 & & 141 & 568 & & 0,37 & 1,49 \\
\hline 676 & w & 1 & gut & 8,9 & 30,0 & 375 & & 93 & 335 & & 0,25 & 0,89 \\
\hline 693 & w & 1 & schlecht & 13,1 & 16,6 & 382 & & 111 & 622 & & 0,29 & 1,63 \\
\hline 696 & $\mathrm{~m}$ & 1 & schlecht & & & 398 & 168 & 46 & 509 & 2,36 & 0,11 & 1,28 \\
\hline 697 & w & 0 & gut & & & 368 & 161 & 89 & 302 & 2,28 & 0,24 & 0,82 \\
\hline 699 & w & 0 & schlecht & & & 407 & 163 & 98 & 558 & 2,50 & 0,24 & 1,37 \\
\hline 704 & $\mathrm{~m}$ & 0 & gut & & & 378 & 169 & 96 & 369 & 2,24 & 0,25 & 0,97 \\
\hline 709 & $\mathrm{~m}$ & 0 & schlecht & & & 380 & 168 & 93 & 523 & 2,26 & 0,24 & 1,38 \\
\hline 711 & w & 1 & schlecht & 22,5 & 24,0 & 380 & & 88 & 499 & & 0,23 & 1,31 \\
\hline 727 & w & 0 & schlecht & 13,6 & 18,1 & 379 & & 127 & 581 & & 0,33 & 1,53 \\
\hline 730 & w & 0 & schlecht & & & 366 & 173 & 126 & 521 & 2,11 & 0,34 & 1,42 \\
\hline 732 & w & 0 & gut & 39,3 & 33,7 & 368 & & 42 & 311 & & 0,11 & 0,85 \\
\hline 733 & $\mathrm{~m}$ & 1 & gut & & & 372 & 165 & 52 & 286 & 2,25 & 0,14 & 0,77 \\
\hline 737 & w & 1 & schlecht & 25,4 & 23,5 & 375 & & 106 & 471 & & 0,28 & 1,26 \\
\hline 743 & $\mathrm{~m}$ & 0 & schlecht & & & 364 & 171 & 103 & 494 & 2,13 & 0,28 & 1,36 \\
\hline 748 & $\mathrm{~m}$ & 1 & schlecht & & & 392 & 173 & 86 & 507 & 2,27 & 0,22 & 1,29 \\
\hline 751 & w & 1 & schlecht & & & 374 & 169 & 117 & 515 & 2,21 & 0,31 & 1,38 \\
\hline 757 & $\mathrm{~m}$ & 1 & schlecht & & & 330 & 152 & 96 & 478 & 2,17 & 0,29 & 1,45 \\
\hline 758 & $\mathrm{~m}$ & 0 & schlecht & & & 391 & 169 & 129 & 527 & 2,31 & 0,33 & 1,35 \\
\hline 764 & w & 0 & schlecht & & & 374 & 170 & 65 & 629 & 2,21 & 0,17 & 1,68 \\
\hline 769 & w & 1 & schlecht & & & 388 & 170 & 76 & 526 & 2,29 & 0,20 & 1,35 \\
\hline 770 & w & 1 & schlecht & 26,8 & 16,4 & 382 & & 82 & 518 & & 0,22 & 1,36 \\
\hline 781 & $\mathrm{~m}$ & 0 & schlecht & & & 351 & 164 & 84 & 533 & 2,14 & 0,24 & 1,52 \\
\hline 785 & $\mathrm{~m}$ & 0 & gut & & & 367 & 162 & 83 & 543 & 2,27 & 0,23 & 1,48 \\
\hline 790 & w & 1 & gut & & & 378 & 166 & 79 & 266 & 2,29 & 0,21 & 0,70 \\
\hline 797 & w & 1 & schlecht & & & 367 & 171 & 130 & 588 & 2,15 & 0,35 & 1,60 \\
\hline 799 & $\mathrm{~m}$ & 0 & schlecht & & & 365 & 170 & 100 & 509 & 2,15 & 0,27 & 1,39 \\
\hline \multicolumn{13}{|l|}{ Sex: } \\
\hline Rang: & & $y-$ & Eler rans & mo & & & & & & & & \\
\hline Ätzen: & & Mas & & & . & and & $\ddot{n}$ & & & & & \\
\hline Verasc & & Mass & erlust bein & rase & & & & & & & & \\
\hline Leerfelc & & keine & aten verfügh & & & & & & & & & \\
\hline
\end{tabular}

Tabelle 26: Kirchheim unter Teck - Probenliste und auswertungsrelevante Individualdaten.

\begin{tabular}{|c|c|c|c|c|c|c|c|c|}
\hline Grab & Histologie & $\begin{array}{c}\mathbf{C a} \\
{[\mathbf{m g} / \mathbf{g}]}\end{array}$ & $\begin{array}{c}\mathbf{P} \\
{[\mathbf{m g} / \mathbf{g}]}\end{array}$ & $\begin{array}{c}\mathbf{B a} \\
{[\mathbf{\mu g} / \mathbf{g}]}\end{array}$ & $\begin{array}{c}\mathbf{S r} \\
{[\mathbf{\mu g} / \mathbf{g}]}\end{array}$ & $\mathbf{C a} / \mathbf{P}$ & $\begin{array}{c}\mathbf{B a} / \mathbf{C a} \\
{\left[\times \mathbf{1 0}^{-3}\right]}\end{array}$ & $\begin{array}{c}\mathbf{S r} / \mathbf{C a} \\
{\left[\times \mathbf{1 0}^{-\mathbf{3}}\right]}\end{array}$ \\
\hline 1 & schlecht & 412 & 165 & 77 & 148 & 2,50 & 0,19 & 0,36 \\
2 & schlecht & 369 & 169 & 91 & 181 & 2,18 & 0,25 & 0,49 \\
3 & schlecht & 354 & 179 & 80 & 124 & 1,97 & 0,23 & 0,35 \\
5 & schlecht & 394 & 170 & 116 & 193 & 2,32 & 0,29 & 0,49 \\
6 & gut & 371 & 155 & 71 & 174 & 2,39 & 0,19 & 0,47 \\
7 & gut & 359 & 161 & 52 & 162 & 2,23 & 0,14 & 0,45 \\
9 & schlecht & 386 & 173 & 64 & 197 & 2,23 & 0,17 & 0,51 \\
11 & schlecht & 422 & 168 & 76 & 165 & 2,52 & 0,18 & 0,39 \\
12 & schlecht & 403 & 171 & 89 & 178 & 2,36 & 0,22 & 0,44 \\
14 & gut & 409 & 168 & 54 & 160 & 2,44 & 0,13 & 0,39 \\
15 & gut & 417 & 175 & 46 & 142 & 2,39 & 0,11 & 0,34 \\
17 & schlecht & 392 & 166 & 84 & 165 & 2,35 & 0,21 & 0,42 \\
18 & schlecht & 441 & 176 & 121 & 150 & 2,50 & 0,27 & 0,34 \\
19 & schlecht & 381 & 168 & 82 & 194 & 2,26 & 0,22 & 0,51 \\
20 & schlecht & 353 & 166 & 73 & 194 & 2,13 & 0,21 & 0,55 \\
\hline
\end{tabular}


Fortsetzung Tabelle 26

\begin{tabular}{|c|c|c|c|c|c|c|c|c|}
\hline Grab & Histologie & $\begin{array}{c}\mathrm{Ca} \\
{[\mathrm{mg} / \mathrm{g}]}\end{array}$ & $\begin{array}{c}P \\
{[\mathrm{mg} / \mathrm{g}]}\end{array}$ & $\begin{array}{c}\text { Вa } \\
{[\mu g / g]}\end{array}$ & $\begin{array}{c}\mathrm{Sr} \\
{[\mu \mathrm{g} / \mathrm{g}]}\end{array}$ & $\mathrm{Ca} / \mathrm{P}$ & $\begin{array}{l}\mathrm{Ba} / \mathrm{Ca} \\
{\left[\times 10^{-3}\right]}\end{array}$ & $\begin{array}{l}\mathrm{Sr} / \mathrm{Ca} \\
{\left[\times 10^{-3}\right]}\end{array}$ \\
\hline 22 & schlecht & 359 & 185 & 68 & 169 & 1,94 & 0,19 & 0,47 \\
\hline 24 & schlecht & 421 & 178 & 103 & 215 & 2,36 & 0,25 & 0,51 \\
\hline 32 & gut & 374 & 162 & 92 & 168 & 2,31 & 0,24 & 0,45 \\
\hline 38 & schlecht & 371 & 177 & 72 & 156 & 2,10 & 0,19 & 0,42 \\
\hline 39 & gut & 381 & 179 & 66 & 137 & 2,13 & 0,17 & 0,36 \\
\hline 40 & gut & 368 & 165 & 79 & 165 & 2,22 & 0,21 & 0,45 \\
\hline 43 & schlecht & 404 & 172 & 162 & 226 & 2,36 & 0,40 & 0,56 \\
\hline 46 & gut & 425 & 164 & 62 & 191 & 2,59 & 0,15 & 0,45 \\
\hline 49 & schlecht & 264 & 176 & 90 & 193 & 1,50 & 0,34 & 0,73 \\
\hline 50 & gut & 394 & 166 & 48 & 169 & 2,38 & 0,12 & 0,43 \\
\hline 51 & schlecht & 367 & 169 & 94 & 205 & 2,17 & 0,26 & 0,56 \\
\hline 52 & gut & 363 & 171 & 83 & 142 & 2,12 & 0,23 & 0,39 \\
\hline 53 & schlecht & 393 & 172 & 139 & 193 & 2,29 & 0,35 & 0,49 \\
\hline 54 & gut & 343 & 158 & 82 & 175 & 2,18 & 0,24 & 0,51 \\
\hline 56 & schlecht & 405 & 167 & 99 & 170 & 2,42 & 0,25 & 0,42 \\
\hline 58 & schlecht & 374 & 165 & 103 & 187 & 2,27 & 0,28 & 0,5 \\
\hline 59 & schlec & 379 & 167 & 142 & 231 & 2,27 & 0,38 &, 61 \\
\hline 61 & schlecht & 486 & 173 & 83 & 190 & 2,81 & 0,17 &, 39 \\
\hline 62 & schlecht & 327 & 161 & 89 & 154 & 2,03 & 0,27 & 0,47 \\
\hline 66 & out & 356 & 167 & 54 & 174 & 2,13 & 0,15 & 0,49 \\
\hline 67 & gut & 370 & 179 & 95 & 192 & 2,07 & 0,26 & 0,52 \\
\hline 68 & schlecht & 423 & 171 & 104 & 203 & 2,48 & 0,25 & 0,48 \\
\hline 72 & gut & 398 & 168 & 75 & 155 & 2,37 & 0,19 & 0,39 \\
\hline 73 & chlect & 393 & 166 & 127 & 212 & 2,37 & 0,32 &, 54 \\
\hline 75 & schlecht & 402 & 171 & 176 & 217 & 2,35 & 0,44 & 0,54 \\
\hline 76 & lecht & 387 & 170 & 97 & 178 & 2,27 & 0,25 & 0,46 \\
\hline 77 & ans & & 173 & 79 & 178 & 2,05 & 0,22 & 0,5 \\
\hline 78 & gut & 349 & 159 & 59 & 136 & 2,20 & 0,17 & 0,39 \\
\hline 79 & gut & 423 & 168 & 76 & 152 & 2,52 & 0,18 & 0,36 \\
\hline 80 & schlecht & 373 & 172 & 106 & 284 & 2,17 & 0,28 & 0,76 \\
\hline 81 & gut & 382 & 164 & 62 & 176 & 2,33 & 0,16 & 0,46 \\
\hline 85 & gut & 359 & 177 & 66 & 187 & 2,03 & 0,18 & 0,52 \\
\hline 87 & schlecht & 363 & 174 & 60 & 196 & 2,08 & 0,17 & 0,54 \\
\hline 89 & schlecht & 90 & 173 & 132 & 195 & 2,26 & 0,34 & 0,5 \\
\hline 90 & lecht & 357 & 170 & 94 & 200 & 2,10 & 0,26 & 0,56 \\
\hline 92 & schlecht & 399 & 173 & 98 & 171 & 2,31 & 0,25 & 0,43 \\
\hline 93 & $\mathrm{t}$ & 374 & 164 & 97 & 179 & 2,28 & 0,26 & 0,48 \\
\hline 94 & schlecht & 346 & 171 & 73 & 221 & 2,02 & 0,21 & 0,64 \\
\hline 95 & schlecht & 355 & 168 & 87 & 177 & 2,11 & 0,25 & 0,5 \\
\hline 96 & $x^{2}$ & & 17 & 93 & 153 & 2,06 & 0,26 & 0,43 \\
\hline 99 & hlecht & 366 & 170 & 95 & 150 & 2,15 & 0,26 & 0,41 \\
\hline 100 & gut & 346 & 172 & 65 & 159 & 2,01 & 0,19 & 0,46 \\
\hline 105 & & & 167 & 74 & 187 & 2,03 & 0,22 & 0,55 \\
\hline 106 & schlech & & 17 & 83 & 17 & 2,28 & 0,21 & 0,45 \\
\hline 107 & schlecht & 342 & 180 & 97 & 212 & 1,90 & 0,28 & 0,62 \\
\hline 108 & crblesht & 440 & 182 & 100 & 194 & 2,41 & 0,23 & 0,44 \\
\hline 109 & sch & & 184 & 112 & 179 & 1,95 & 0,31 & 0.5 \\
\hline 111 & - & 377 & 170 & 64 & 192 & 2,22 & 0,17 & 0,51 \\
\hline 112 & schlecht & 427 & 191 & 124 & 235 & 2,23 & 0,29 & 0,55 \\
\hline 113 & coblesht & & 175 & 70 & 148 & 2,02 & 0,20 & 0,42 \\
\hline 114 & & & $17 \mathrm{H}-2$ & 12 & 210 & 2,17 & 0,34 & 0,57 \\
\hline 115 & $s$ & 379 & 174 & 108 & 144 & 2,18 & 0,28 & 0,38 \\
\hline 116 & - & 438 & 169 & 72 & 166 & 2,58 & 0,16 & 0,38 \\
\hline 117 & $\mathrm{scl}$ & & 171 & 104 & 169 & 2,24 & 0,27 & 0,44 \\
\hline 118 & $x_{1}+2>$ & & 17 & 75 & 169 & 2,08 & 0,21 & 0,47 \\
\hline 120 & schlecht & 36 & 173 & 102 & 195 & 2,13 & 0,28 & 0,53 \\
\hline 123 & 100 & & 175 & 61 & 23 & 2,24 & 0,15 & 0,61 \\
\hline 130 & 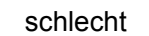 & 39 & 17 & 87 & 166 & 2,25 & 0,22 & 0,42 \\
\hline 132 & schlecht & 366 & 173 & 101 & 187 & 2,11 & 0,28 & 0,51 \\
\hline 133 & schlecht & 383 & 176 & 99 & 207 & 2,17 & 0,26 & 0,54 \\
\hline 136 & & 408 & 18 & 70 & 159 & 2,19 & 0,17 & 0,39 \\
\hline 137 & schlecht & 360 & 171 & 82 & 101 & 2,10 & 0,23 & 0,28 \\
\hline 138 & on & 431 & 167 & 59 & 138 & 2,58 & 0,14 & 0,32 \\
\hline 141 & schlech & 365 & 178 & 95 & 193 & 2,05 & 0,26 & 0,53 \\
\hline 143 & schlec & 345 & 16 & 55 & 176 & 2,09 & 0,16 & 0,51 \\
\hline 145 & schlecht & 360 & 177 & 51 & 216 & 2,03 & 0,14 & 0,6 \\
\hline 146 & gut & 363 & 162 & 50 & 167 & 2,23 & 0,14 & 0,46 \\
\hline 147 & . & 343 & 183 & 59 & 141 & 1,87 & 0,17 & 0,41 \\
\hline 150 & gut & 334 & 174 & 65 & 194 & 1,92 & 0,19 & 0,58 \\
\hline 152 & schlecht & 354 & 169 & 94 & 238 & 2,09 & 0,27 & 0,67 \\
\hline 154 & schlecht & 371 & 187 & 83 & 253 & 1,99 & 0,22 & 0,68 \\
\hline
\end{tabular}


Fortsetzung Tabelle 26

\begin{tabular}{|c|c|c|c|c|c|c|c|c|}
\hline Grab & Histologie & $\begin{array}{c}\mathbf{C a} \\
{[\mathbf{m g} / \mathbf{g}]}\end{array}$ & $\begin{array}{c}\mathbf{P} \\
{[\mathbf{m g} / \mathbf{g}]}\end{array}$ & $\begin{array}{c}\mathbf{B a} \\
{[\mathbf{\mu g} / \mathbf{g}]}\end{array}$ & $\begin{array}{c}\mathbf{S r} \\
{[\mathbf{\mu g} / \mathbf{g}]}\end{array}$ & $\mathbf{C a / P}$ & $\begin{array}{c}\mathbf{B a} / \mathbf{C a} \\
{\left[\times \mathbf{1 0}^{-3}\right]}\end{array}$ & $\begin{array}{c}\mathbf{S r} / \mathbf{C a} \\
{\left[\times \mathbf{1 0 ^ { - 3 }}\right]}\end{array}$ \\
\hline 155 & schlecht & 411 & 183 & 140 & 169 & 2,24 & 0,34 & 0,41 \\
156 & schlecht & 354 & 179 & 103 & 227 & 1,98 & 0,29 & 0,64 \\
157 & schlecht & 484 & 176 & 120 & 223 & 2,75 & 0,25 & 0,46 \\
161 & schlecht & 349 & 182 & 86 & 143 & 1,92 & 0,25 & 0,41 \\
162 & schlecht & 385 & 204 & 109 & 173 & 1,89 & 0,28 & 0,45 \\
165 & schlecht & 340 & 157 & 87 & 180 & 2,17 & 0,26 & 0,53 \\
166 & schlecht & 394 & 182 & 57 & 174 & 2,17 & 0,15 & 0,44 \\
167 & schlecht & 357 & 180 & 52 & 175 & 1,98 & 0,15 & 0,49 \\
168 & gut & 351 & 178 & 34 & 155 & 1,97 & 0,10 & 0,44 \\
169 & schlecht & 363 & 186 & 74 & 189 & 1,96 & 0,20 & 0,52 \\
172 & schlecht & 391 & 178 & 73 & 145 & 2,19 & 0,19 & 0,37 \\
174 & schlecht & 382 & 183 & 68 & 183 & 2,08 & 0,18 & 0,48 \\
175 & schlecht & 379 & 190 & 84 & 186 & 1,99 & 0,22 & 0,49 \\
176 & schlecht & 368 & 174 & 92 & 180 & 2,11 & 0,25 & 0,49 \\
177 & gut & 361 & 177 & 73 & 137 & 2,03 & 0,20 & 0,38 \\
\hline Átzen:
\end{tabular}

Veraschen: Masseverlust beim Veraschen

Tabelle 27: Wenigumstadt - Probenliste und auswertungsrelevante Individualdaten.

\begin{tabular}{|c|c|c|c|c|c|c|c|c|c|c|}
\hline \multirow[t]{2}{*}{ Grab } & \multirow[t]{2}{*}{ Histologie } & \multicolumn{2}{|c|}{ Masseverlust [\%] } & \multirow{2}{*}{$\begin{array}{c}\mathrm{Ca} \\
{[\mathrm{mg} / \mathrm{g}]}\end{array}$} & \multirow{2}{*}{$\begin{array}{c}P \\
{[\mathrm{mg} / \mathrm{g}]}\end{array}$} & \multirow{2}{*}{$\begin{array}{c}\mathrm{Ba} \\
{[\mu \mathrm{g} / \mathrm{g}]}\end{array}$} & \multirow{2}{*}{$\begin{array}{c}\mathrm{Sr} \\
{[\mu \mathrm{g} / \mathrm{g}]}\end{array}$} & \multirow[t]{2}{*}{$\mathrm{Ca} / \mathrm{P}$} & \multirow{2}{*}{$\begin{array}{l}\mathrm{Ba} / \mathrm{Ca} \\
{\left[\times 10^{-3}\right]}\end{array}$} & \multirow{2}{*}{$\begin{array}{l}\mathrm{Sr} / \mathrm{Ca} \\
{\left[\times 10^{-3}\right]}\end{array}$} \\
\hline & & Ätzen & Veraschen & & & & & & & \\
\hline 10 & schlecht & 11,8 & 13,1 & 374 & 168 & 79 & 273 & 2,22 & 0,21 & 0,73 \\
\hline 11 & schlecht & 14,4 & 12,8 & 374 & 158 & 81 & 338 & 2,37 & 0,22 & 0,90 \\
\hline 12 & schlecht & 11,3 & 15,9 & 380 & 170 & 96 & 265 & 2,24 & 0,25 & 0,70 \\
\hline 13 & schlecht & 8,4 & 17,4 & 388 & 174 & 59 & 171 & 2,24 & 0,15 & 0,44 \\
\hline 14 & schlecht & 13,2 & 14,4 & 375 & 161 & 73 & 295 & 2,33 & 0,20 & 0,79 \\
\hline 15 & schlecht & 11,1 & 13,7 & 372 & 165 & 69 & 213 & 2,26 & 0,19 & 0,57 \\
\hline 17 & schlecht & 15,0 & 14,1 & 387 & 166 & 114 & 322 & 2,34 & 0,29 & 0,83 \\
\hline 18 & gut & 8,1 & 29,6 & 377 & 159 & 70 & 327 & 2,37 & 0,19 & 0,87 \\
\hline 19 & schlecht & 11,8 & 14,0 & 387 & 165 & 95 & 364 & 2,34 & 0,25 & 0,94 \\
\hline 25 & schlecht & 13,9 & 13,8 & 351 & 144 & 99 & 337 & 2,44 & 0,28 & 0,96 \\
\hline 29 & schlecht & 15,5 & 11,6 & 389 & 167 & 108 & 345 & 2,33 & 0,28 & 0,89 \\
\hline 31 & schlecht & 15,4 & 15,3 & 378 & 157 & 93 & 408 & 2,40 & 0,25 & 1,08 \\
\hline 32 & gut & 7,9 & 31,1 & 378 & 166 & 70 & 255 & 2,28 & 0,19 & 0,67 \\
\hline 34 & gut & 9,5 & 31,6 & 375 & 155 & 76 & 261 & 2,42 & 0,20 & 0,70 \\
\hline 36 & schlecht & 12,6 & 13,2 & 376 & 174 & 115 & 359 & 2,17 & 0,31 & 0,95 \\
\hline 37 & gut & 10,6 & 26,1 & 374 & 163 & 78 & 236 & 2,30 & 0,21 & 0,63 \\
\hline 39 & schlecht & 17,8 & 14,4 & 373 & 152 & 131 & 402 & 2,45 & 0,35 & 1,08 \\
\hline 41 & qut & 11.0 & 28,6 & 375 & 159 & 70 & 334 & 2,36 & 0,19 & 0,89 \\
\hline 42 & schlecht & 12,1 & 12,2 & 387 & 169 & 119 & 387 & 2,30 & 0,31 & 1,00 \\
\hline 44 & schlecht & 12,5 & 18,7 & 381 & 176 & 73 & 404 & 2,16 & 0,19 & 1,06 \\
\hline 47 & gut & 9,1 & 29,1 & 387 & 163 & 77 & 276 & 2,38 & 0,20 & 0,71 \\
\hline 48 & gut & 10,6 & 31,8 & 386 & 166 & 64 & 240 & 2,33 & 0,17 & 0,62 \\
\hline 50 & gut & 14,2 & 28,5 & 387 & 159 & 60 & 266 & 2,43 & 0,15 & 0,69 \\
\hline 52 & schlecht & 12,2 & 13,1 & 388 & 167 & 98 & 348 & 2,32 & 0,25 & 0,90 \\
\hline 53 & schlecht & 13,0 & 13,9 & 358 & 166 & 119 & 425 & 2,16 & 0,33 & 1,19 \\
\hline 96 & schlecht & 15,3 & 20,3 & 376 & 163 & 172 & 495 & 2,30 & 0,46 & 1,32 \\
\hline 111 & gut & 10,4 & 31,0 & 387 & 155 & 81 & 280 & 2,49 & 0,21 & 0,72 \\
\hline 115 & schlecht & 13,7 & 17,5 & 374 & 160 & 215 & 423 & 2,34 & 0,57 & 1,13 \\
\hline 148 & gut & 10,6 & 28,7 & 371 & 162 & 67 & 267 & 2,29 & 0,18 & 0,72 \\
\hline 165 & gut & 8,9 & 30,5 & 346 & 161 & 70 & 273 & 2,14 & 0,20 & 0,79 \\
\hline 170 & schlecht & 11,8 & 15,2 & 379 & & 118 & 383 & & 0,31 & 1,01 \\
\hline 171 & schlecht & 12,5 & 12,5 & 383 & 163 & 110 & 298 & 2,35 & 0,29 & 0,78 \\
\hline 173 & schlecht & 13,6 & 13,9 & 388 & 167 & 107 & 289 & 2,32 & 0,28 & 0,75 \\
\hline 178 & schlecht & 8,5 & 16,9 & 391 & 182 & 58 & 237 & 2,15 & 0,15 & 0,60 \\
\hline 179 & gut & 10,0 & 29,6 & 415 & 175 & 83 & 298 & 2,38 & 0,20 & 0,72 \\
\hline 184 & gut & 7,0 & 30,4 & 390 & 169 & 62 & 229 & 2,32 & 0,16 & 0,59 \\
\hline 185 & schlecht & 9,4 & 17,7 & 386 & & 106 & 337 & & 0,28 & 0,87 \\
\hline 188 & schlecht & 10,0 & 11,9 & 380 & 168 & 82 & 317 & 2,27 & 0,22 & 0,83 \\
\hline 189 & schlecht & 10,2 & 22,4 & 380 & 170 & 37 & 272 & 2,24 & 0,10 & 0,72 \\
\hline 191 & schlecht & 12,0 & 18,4 & 389 & 162 & 102 & 423 & 2,39 & 0,26 & 1,09 \\
\hline 194 & gut & 8,3 & 31,2 & 376 & 165 & 43 & 240 & 2,27 & 0,11 & 0,64 \\
\hline 200 & schlecht & 12,1 & 14,2 & 380 & 162 & 108 & 326 & 2,34 & 0,28 & 0,86 \\
\hline 201 & gut & 9,8 & 32,2 & 370 & 162 & 71 & 275 & 2,29 & 0,19 & 0,74 \\
\hline
\end{tabular}


Fortsetzung Tabelle 27

\begin{tabular}{|c|c|c|c|c|c|c|c|c|c|c|}
\hline \multirow[t]{2}{*}{ Grab } & \multirow[t]{2}{*}{ Histologie } & \multicolumn{2}{|c|}{ Masseverlust [\%] } & \multirow{2}{*}{$\begin{array}{r}\mathrm{Ca} \\
{[\mathrm{mg} / \mathrm{g}]}\end{array}$} & \multirow{2}{*}{$\begin{array}{c}P \\
{[\mathrm{mg} / \mathrm{g}]}\end{array}$} & \multirow{2}{*}{$\begin{array}{c}\mathrm{Ba} \\
{[\mu \mathrm{g} / \mathrm{g}]}\end{array}$} & \multirow{2}{*}{$\begin{array}{c}\mathrm{Sr} \\
{[\mu \mathrm{g} / \mathrm{g}]}\end{array}$} & \multirow[t]{2}{*}{$\mathrm{Ca} / \mathrm{P}$} & \multirow{2}{*}{$\begin{array}{l}\mathrm{Ba} / \mathrm{Ca} \\
{\left[\times 10^{-3}\right]}\end{array}$} & \multirow{2}{*}{$\begin{array}{l}\mathrm{Sr} / \mathrm{Ca} \\
{\left[\times 10^{-3}\right]}\end{array}$} \\
\hline & & Ätzen & Veraschen & & & & & & & \\
\hline 202 & schlecht & 12,8 & 16,4 & 376 & & 127 & 355 & & 0,34 & 0,94 \\
\hline 203 & schlecht & 12,0 & 15,0 & 376 & 168 & 119 & 275 & 2,24 & 0,32 & 0,73 \\
\hline 204 & schlecht & 6,2 & 20,9 & 391 & 176 & 70 & 281 & 2,23 & 0,18 & 0,72 \\
\hline 205 & gut & 5,8 & 31,1 & 390 & & 95 & 307 & & 0,24 & 0,79 \\
\hline 208 & schlecht & 8,2 & 17,4 & 369 & 164 & 107 & 353 & 2,26 & 0,29 & 0,95 \\
\hline 209 & gut & 11,0 & 31,3 & 381 & 164 & 97 & 287 & 2,33 & 0,25 & 0,75 \\
\hline 211 & gut & 6,8 & 31,5 & 376 & 158 & 76 & 287 & 2,38 & 0,20 & 0,76 \\
\hline 212 & schlecht & 9,7 & 18,9 & 384 & 168 & 124 & 386 & 2,29 & 0,32 & 1,01 \\
\hline 216 & schlecht & 10,1 & 13,7 & 387 & 159 & 123 & 335 & 2,44 & 0,32 & 0,87 \\
\hline 217 & schlecht & 9,5 & 13,4 & 384 & 156 & 92 & 400 & 2,46 & 0,24 & 1,04 \\
\hline 218 & gut & 7,3 & 31,9 & 364 & & 73 & 208 & & 0,20 & 0,57 \\
\hline 219 & gut & 6,4 & 31,9 & 364 & & 54 & 182 & & 0,15 & 0,50 \\
\hline 220 & schlecht & 10,8 & 15,0 & 377 & 148 & 160 & 394 & 2,55 & 0,42 & 1,04 \\
\hline 221 & schlecht & 15,6 & 15,7 & 386 & & 72 & 286 & & 0,19 & 0,74 \\
\hline 222 & gut & 6,1 & 30,8 & 383 & 162 & 45 & 258 & 2,36 & 0,12 & 0,67 \\
\hline 224 & schlecht & 14,1 & 16,8 & 411 & 162 & 108 & 358 & 2,54 & 0,26 & 0,87 \\
\hline 225 & schlecht & 8,9 & 15,3 & 363 & 167 & 113 & 362 & 2,18 & 0,31 & 1,00 \\
\hline 226 & schlecht & 9,2 & 13,4 & 383 & 181 & 86 & 231 & 2,11 & 0,23 & 0,60 \\
\hline 231 & schlecht & 13,9 & 13,5 & 378 & 162 & 172 & 466 & 2,34 & 0,45 & 1,23 \\
\hline 234 & gut & 9,1 & 32,4 & 370 & & 82 & 389 & & 0,22 & 1,05 \\
\hline 236 & schlecht & 17,1 & 17,0 & 404 & & 134 & 373 & & 0,33 & 0,92 \\
\hline 238 & schlecht & 15,3 & 26,0 & 386 & 158 & 91 & 332 & 2,45 & 0,24 & 0,86 \\
\hline 239 & schlecht & 6,7 & 20,9 & 374 & 168 & 51 & 276 & 2,22 & 0,14 & 0,74 \\
\hline 240 & gut & 10,1 & 29,5 & 378 & 157 & 105 & 321 & 2,41 & 0,28 & 0,85 \\
\hline 241 & schlecht & 12,8 & 9,2 & 378 & & 124 & 305 & & 0,33 & 0,81 \\
\hline 242 & gut & 12,2 & 29,3 & 406 & & 112 & 278 & & 0,28 & 0,69 \\
\hline 243 & gut & 10,1 & 29,5 & 346 & & 92 & 310 & & 0,27 & 0,90 \\
\hline 244 & schlecht & 14,7 & 13,0 & 377 & 167 & 169 & 392 & 2,26 & 0,45 & 1,04 \\
\hline 245 & schlecht & 9,4 & 15,2 & 362 & 178 & 87 & 315 & 2,03 & 0,24 & 0,87 \\
\hline 248 & schlecht & 12,3 & 17,2 & 353 & 159 & 79 & 285 & 2,22 & 0,22 & 0,81 \\
\hline 249 & & 9,8 & 288 & 379 & 170 & 58 & 28 & 2,24 & 0,15 & 0,75 \\
\hline 251 & schlecht & 11,5 & 16,8 & 367 & 168 & 69 & 301 & 2,18 & 0,19 & 0,82 \\
\hline 252 & schlecht & 15,0 & 17,9 & 398 & 150 & 135 & 384 & 2,65 & 0,34 & 0,97 \\
\hline 253 & schlecht & 13,3 & 16,8 & 366 & & 165 & 386 & & 0,45 & 1,06 \\
\hline 254 & schlecht & 13,3 & 15,9 & 343 & 170 & 103 & 441 & 2,02 & 0,30 & 1,29 \\
\hline 255 & gut & 8,4 & 29,5 & 362 & & 44 & 214 & & 0,12 & 0,59 \\
\hline 256 & schlecht & 10,0 & 14,4 & 363 & & 108 & 328 & & 0,30 & 0,90 \\
\hline 258 & schlecht & 14,2 & 17,4 & 372 & 165 & 108 & 362 & 2,26 & 0,29 & 0,97 \\
\hline 260 & gut & 11,6 & 28,8 & 394 & 166 & 61 & 276 & 2,37 & 0,16 & 0,70 \\
\hline 261 & schlecht & 13,1 & 16,3 & 382 & & 155 & 394 & & 0,41 & 1,03 \\
\hline 262 & schlecht & 11,4 & 14,7 & 379 & 164 & 170 & 334 & 2,31 & 0,45 & 0,88 \\
\hline 263 & schlecht & 9,3 & 13,4 & 381 & 170 & 86 & 278 & 2,25 & 0,23 & 0,73 \\
\hline 264 & gut & 7,6 & 31,3 & 375 & 163 & 59 & 284 & 2,30 & 0,16 & 0,76 \\
\hline
\end{tabular}


Tabelle 28: Kirchheim am Ries - Auswertungsrelevante Individualdaten für den unbehandelten Probensatz.

\begin{tabular}{|c|c|c|c|c|c|c|c|c|c|c|c|c|}
\hline Grab & Histologie & $\begin{array}{c}\mathrm{Ca} \\
{[\mathrm{mg} / \mathrm{g}]}\end{array}$ & $\begin{array}{c}P \\
{[\mathrm{mg} / \mathrm{g}]}\end{array}$ & $\begin{array}{c}\mathrm{Ba} \\
{[\mu \mathrm{g} / \mathrm{g}]}\end{array}$ & $\begin{array}{c}\mathrm{Sr} \\
{[\mu \mathrm{g} / \mathrm{g}]}\end{array}$ & $\begin{array}{c}M g \\
{[\mu g / g]}\end{array}$ & $\begin{array}{c}\text { Al } \\
{[\mu g / g]}\end{array}$ & $\mathrm{Ca} / \mathrm{P}$ & $\begin{array}{l}\mathrm{Ba} / \mathrm{Ca} \\
{\left[\times 10^{-3}\right]}\end{array}$ & $\begin{array}{l}\mathrm{Sr} / \mathrm{Ca} \\
{\left[\times 10^{-3}\right]}\end{array}$ & $\begin{array}{l}\mathrm{Mg} / \mathrm{Ca} \\
{\left[\times 10^{-3}\right]}\end{array}$ & $\begin{array}{l}\mathrm{Al} / \mathrm{Ca} \\
{\left[\times 10^{-3}\right]}\end{array}$ \\
\hline 52 & gut & 374 & 159 & 42 & 132 & 1053 & 107 & 2,36 & 0,11 & 0,35 & 2,81 & 0,29 \\
\hline 73 & schlecht & 377 & 162 & 54 & 140 & 879 & 65 & 2,32 & 0,14 & 0,37 & 2,33 & 0,17 \\
\hline 73,1 & schlecht & 379 & 160 & 59 & 163 & 757 & 88 & 2,37 & 0,16 & 0,43 & 2,00 & 0,23 \\
\hline 74 & gut & 369 & 158 & 37 & 90 & 819 & 38 & 2,34 & 0,10 & 0,24 & 2,22 & 0,10 \\
\hline 81 & schlecht & 377 & 165 & 41 & 130 & 866 & 541 & 2,28 & 0,11 & 0,34 & 2,30 & 1,43 \\
\hline 84,1 & schlecht & 377 & 164 & 68 & 135 & 690 & 205 & 2,31 & 0,18 & 0,36 & 1,83 & 0,54 \\
\hline 92 & schlecht & 376 & 156 & 87 & 184 & 661 & 152 & 2,41 & 0,23 & 0,49 & 1,76 & 0,40 \\
\hline 106 & gut & 373 & 156 & 69 & 130 & 1131 & 172 & 2,39 & 0,18 & 0,35 & 3,03 & 0,46 \\
\hline 115 & gut & 373 & 159 & 32 & 119 & 946 & 134 & 2,35 & 0,09 & 0,32 & 2,54 & 0,36 \\
\hline 133,1 & schlecht & 379 & 162 & 105 & 168 & 783 & 526 & 2,34 & 0,28 & 0,44 & 2,07 & 1,39 \\
\hline 149 & gut & 372 & 158 & 32 & 117 & 970 & 53 & 2,36 & 0,09 & 0,32 & 2,61 & 0,14 \\
\hline 152 & schlecht & 380 & 155 & 109 & 191 & 741 & 541 & 2,45 & 0,29 & 0,50 & 1,95 & 1,42 \\
\hline 159 & schlecht & 380 & 160 & 98 & 238 & 602 & 147 & 2,37 & 0,26 & 0,63 & 1,58 & 0,39 \\
\hline 166 & gut & 371 & 157 & 47 & 130 & 1532 & 91 & 2,37 & 0,13 & 0,35 & 4,13 & 0,24 \\
\hline 167 & schlecht & 377 & 160 & 64 & 165 & 660 & 103 & 2,35 & 0,17 & 0,44 & 1,75 & 0,27 \\
\hline 168 & gut & 376 & 161 & 61 & 163 & 1467 & 63 & 2,33 & 0,16 & 0,43 & 3,90 & 0,17 \\
\hline 172 & gut & 378 & 160 & 29 & 114 & 1377 & 40 & 2,36 & 0,08 & 0,30 & 3,64 & 0,11 \\
\hline 178 & gut & 377 & 160 & 59 & 155 & 1001 & 119 & 2,36 & 0,16 & 0,41 & 2,66 & 0,32 \\
\hline 185 & schlecht & 377 & 161 & 71 & 173 & 1000 & 100 & 2,34 & 0,19 & 0,46 & 2,65 & 0,26 \\
\hline 206 & schlecht & 385 & 164 & 68 & 163 & 628 & 17 & 2,35 & 0,18 & 0,42 & 1,63 & 0,04 \\
\hline 211 & schlecht & 383 & 162 & 72 & 146 & 709 & 208 & 2,36 & 0,19 & 0,38 & 1,85 & 0,54 \\
\hline 216 & gut & 375 & 158 & 43 & 113 & 1315 & 93 & 2,38 & 0,11 & 0,30 & 3,51 & 0,25 \\
\hline 233,1 & schlecht & 388 & 169 & 46 & 152 & 795 & 211 & 2,30 & 0,12 & 0,39 & 2,05 & 0,54 \\
\hline 235 & schlecht & 380 & 162 & 79 & 167 & 688 & 174 & 2,35 & 0,21 & 0,44 & 1,81 & 0,46 \\
\hline 248 & schlecht & 380 & 162 & 70 & 204 & 937 & 384 & 2,34 & 0,19 & 0,54 & 2,47 & 1,01 \\
\hline 263 & schlecht & 387 & 164 & 81 & 168 & 740 & 128 & 2,35 & 0,21 & 0,43 & 1,91 & 0,33 \\
\hline 268 & gut & 385 & 164 & 48 & 126 & 1099 & 384 & 2,34 & 0,13 & 0,33 & 2,86 & 1,00 \\
\hline 298 & schlecht & 384 & 169 & 52 & 131 & 880 & 103 & 2,27 & 0,14 & 0,34 & 2,29 & 0,27 \\
\hline 304 & gut & 376 & 161 & 45 & 123 & 1042 & 101 & 2,34 & 0,12 & 0,33 & 2,77 & 0,27 \\
\hline 311,1 & schlecht & 382 & 165 & 116 & 233 & 824 & 333 & 2,31 & 0,30 & 0,61 & 2,16 & 0,87 \\
\hline 319,2 & schlecht & 367 & 160 & 71 & 172 & 813 & 445 & 2,29 & 0,19 & 0,47 & 2,21 & 1,21 \\
\hline 321,1 & gut & 371 & 160 & 33 & 145 & 1489 & 36 & 2,31 & 0,09 & 0,39 & 4,02 & 0,10 \\
\hline 345 & schlecht & 370 & 157 & 147 & 230 & 787 & 300 & 2,36 & 0,40 & 0,62 & 2,13 & 0,81 \\
\hline 350 & gut & 367 & 158 & 41 & 155 & 1011 & 123 & 2,32 & 0,11 & 0,42 & 2,75 & 0,34 \\
\hline 358 & schlecht & 367 & 165 & 54 & 173 & 740 & 39 & 2,23 & 0,15 & 0,47 & 2,02 & 0,11 \\
\hline 374 & schlecht & 371 & 162 & 56 & 229 & 865 & 148 & 2,29 & 0,15 & 0,62 & 2,33 & 0,40 \\
\hline 375 & schlecht & 372 & 161 & 60 & 144 & 803 & 106 & 2,31 & 0,16 & 0,39 & 2,16 & 0,29 \\
\hline 380 & schlecht & 373 & 155 & 114 & 203 & 710 & 405 & 2,40 & 0,31 & 0,54 & 1,90 & 1,09 \\
\hline 384 & schlecht & 375 & 164 & 50 & 142 & 714 & 52 & 2,29 & 0,13 & 0,38 & 1,90 & 0,14 \\
\hline 402 & gut & 371 & 162 & 35 & 135 & 1493 & 82 & 2,29 & 0,09 & 0,36 & 4,02 & 0,22 \\
\hline 425 & gut & 378 & 165 & 46 & 129 & 980 & 90 & 2,28 & 0,12 & 0,34 & 2,60 & 0,24 \\
\hline 430 & schlecht & 380 & 164 & 74 & 159 & 773 & 189 & 2,32 & 0,19 & 0,42 & 2,03 & 0,50 \\
\hline 434 & gut & 378 & 166 & 23 & 101 & 970 & 115 & 2,27 & 0,06 & 0,27 & 2,57 & 0,31 \\
\hline 444 & schlecht & 385 & 166 & 59 & 200 & 706 & 162 & 2,32 & 0,15 & 0,52 & 1,83 & 0,42 \\
\hline 455 & schlecht & 379 & 163 & 81 & 188 & 620 & 230 & 2,33 & 0,21 & 0,49 & 1,63 & 0,61 \\
\hline 456 & schlecht & 376 & 162 & 82 & 170 & 731 & 191 & 2,33 & 0,22 & 0,45 & 1,95 & 0,51 \\
\hline 458 & gut & 373 & 162 & 51 & 143 & 987 & 122 & 2,30 & 0,14 & 0,38 & 2,65 & 0,33 \\
\hline 459 & schlecht & 378 & 166 & 50 & 154 & 763 & 55 & 2,28 & 0,13 & 0,41 & 2,02 & 0,15 \\
\hline
\end{tabular}


Tabelle 29: Kirchheim am Ries - Auswertungsrelevante Individualdaten für den Probensatz, der nur geätzt wurde.

\begin{tabular}{|c|c|c|c|c|c|c|c|c|c|c|c|c|c|c|}
\hline \multirow[t]{2}{*}{ Grab } & \multirow[t]{2}{*}{ Histologie } & \multicolumn{2}{|c|}{ Masseverlust [\%] } & \multirow{2}{*}[\begin{array}{c}{\mathrm{Ca}}\\
{[\mathrm{mg}/\mathrm{g}]}\end{array}]{} & \multirow{2}{*}{$\begin{array}{c}P \\
\mathrm{mg} / \mathrm{g}]\end{array}$} & \multirow{2}{*}{$\begin{array}{c}\mathrm{Ba} \\
{[\mu \mathrm{g} / \mathrm{g}]}\end{array}$} & \multirow{2}{*}{$\begin{array}{c}\mathrm{Sr} \\
{[\mu \mathrm{g} / \mathrm{g}]}\end{array}$} & \multirow{2}{*}{$\begin{array}{c}\mathrm{Mg} \\
{[\mu \mathrm{g} / \mathrm{g}]}\end{array}$} & \multirow{2}{*}{$\begin{array}{c}\mathrm{Al} \\
{[\mu \mathrm{g} / \mathrm{g}]}\end{array}$} & \multirow[t]{2}{*}{$\mathrm{Ca} / \mathrm{P}$} & \multirow{2}{*}{$\begin{array}{l}\mathrm{Ba} / \mathrm{Ca} \\
{\left[\times 10^{-3}\right]}\end{array}$} & \multirow{2}{*}{$\begin{array}{l}\mathrm{Sr} / \mathrm{Ca} \\
{\left[\times 10^{-3}\right]}\end{array}$} & \multirow{2}{*}{$\begin{array}{l}\mathrm{Mg} / \mathrm{Ca} \\
{\left[\times 10^{-3}\right]}\end{array}$} & \multirow{2}{*}{$\begin{array}{l}\mathrm{Al} / \mathrm{Ca} \\
{\left[\times 10^{-3}\right]}\end{array}$} \\
\hline & & Ätzen & Veraschen & & & & & & & & & & & \\
\hline 52 & gut & 19,0 & 29,8 & 365 & 164 & 37 & 125 & 1199 & 18 & 2,23 & 0,10 & 0,34 & 3,29 & 0,05 \\
\hline 73 & schlecht & 6,8 & 14,3 & 361 & 167 & 50 & 129 & 854 & 41 & 2,16 & 0,14 & 0,36 & 2,36 & 0,11 \\
\hline 73,1 & schlecht & 24,1 & 13,8 & 364 & 166 & 56 & 153 & 733 & 85 & 2,20 & 0,15 & 0,42 & 2,01 & 0,23 \\
\hline 74 & gut & 19,7 & 29,0 & 361 & 165 & 36 & 87 & 891 & 26 & 2,19 & 0,10 & 0,24 & 2,47 & 0,07 \\
\hline 81 & schlecht & 24,5 & 13,7 & 368 & 168 & 59 & 129 & 674 & 163 & 2,20 & 0,16 & 0,35 & 1,83 & 0,44 \\
\hline 84,1 & schlecht & 25,5 & 12,0 & 372 & 169 & 68 & 132 & 675 & 250 & 2,19 & 0,18 & 0,35 & 1,82 & 0,67 \\
\hline 92 & schlecht & 21,8 & 17,2 & 369 & 164 & 86 & 180 & 655 & 156 & 2,25 & ,23 & 0,49 & 1,77 & 0,42 \\
\hline 106 & gut & 20,9 & & 369 & 165 & 68 & 125 & 1190 & 57 & 2,24 & 0,18 & 0,34 & 3,22 & 0,15 \\
\hline 115 & & 21,2 & 28,7 & 366 & 167 & 29 & 114 & 929 & 87 & 2,19 & ,08 & 0,31 & 2,54 & 0,24 \\
\hline 133,1 & schlecht & 44,6 & 14,1 & 363 & 173 & 91 & 147 & 736 & 569 & 2,10 &, 25 & 0,41 & 2,03 & 1,57 \\
\hline 149 & & 21,0 & 26,6 & 365 & 164 & 33 & 117 & 1054 & 13 & 2,22 &, 09 & 0,32 & 2,89 & 0,03 \\
\hline 152 & niec & 30,7 & & 369 & 167 & 110 & 182 & 723 & 669 & 2,21 & 0 & & 96 & 1,82 \\
\hline 159 & & 24 & 1 & 374 & 167 & 94 & 231 & 577 & 134 & 2,24 & 5 & 0,62 & & 0,36 \\
\hline 166 & & 12 & 25 & 365 & 162 & 47 & 128 & 1620 & 73 & 2,25 & 13 & 0,35 & 4,44 & 0,20 \\
\hline 167 & ecl & 22 & & & 168 & 63 & 166 & 618 & 67 & 2,20 & 7 & & 1,68 & 0,18 \\
\hline 168 & & & & & 166 & & 151 & 1526 & 35 & 2,20 & & & & 0,10 \\
\hline 172 & & 1 & ? & 363 & 162 & 30 & 112 & 1419 & 11 & 2,24 & 08 & & 3,91 & 0,03 \\
\hline 178 & & 1 & & 367 & 164 & 56 & 151 & 1007 & 62 & 2,23 & 5 & 0,41 & 2,75 & 0,17 \\
\hline 185 & hlecht & 1 & & 3 & 167 & 73 & 178 & 978 & 121 & 2,20 & 0 & 0 & 6 & 0,33 \\
\hline 206 & & 2 & 14 & 36 & 168 & 64 & 156 & 617 & 0 & 2,20 & 7 & 0 & 37 & 0,00 \\
\hline 211 & blecht & 7 & 1 & 375 & 175 & 63 & 160 & 722 & 544 & 2,15 &, 17 & 0,43 & 1,93 & 1,45 \\
\hline 216 & & & & & 163 & 44 & 115 & 1381 & 21 & 2,26 & 2 & & 5 & 0,06 \\
\hline 233,1 & & 2 & & & 17 & 12 & 150 & 819 & 109 & 2,13 & 2 & & & 0,29 \\
\hline 235 & If & 23,6 & & 361 & 167 & 80 & 163 & 677 & 228 & 2,16 & 2 & 0,45 & 1,88 & 0,63 \\
\hline 248 & & & & & 16 & 67 & 201 & 846 & 343 & 2,19 & 8 & & & 0,93 \\
\hline 263 & s & 2 & & & 16 & 76 & & 705 & 44 & 2,22 & 1 & & & 0,12 \\
\hline 268 & & 2 & & & 169 & 40 & 113 & 1028 & 254 & 2,17 & 11 & & 2,81 & 0,69 \\
\hline 298 & hlect & 24,8 & 127 & 367 & 173 & 50 & 127 & 763 & 109 & 2,13 & 0,14 & 0,35 & 2,08 & 0,30 \\
\hline 304 & & & & & 16 & 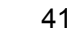 & 116 & 1032 & 23 & 2,19 & & & & 0,06 \\
\hline 311,1 & chlecht & 2 & & 363 & 169 & 113 & 216 & 649 & 404 & 2,15 & 1 & 0,60 & 1,79 & 1,11 \\
\hline 319,2 & schlecht & & & & 174 & 37 & 116 & 788 & 493 & 2,10 & 0 & & 2,17 & 1,36 \\
\hline 321,1 & & & & & & & & 1447 & 0 & 2,26 & & & & 0,00 \\
\hline 345 & (II & & & & 165 & 146 & 21 & 686 & 316 & 2,22 & 0 & 0, & 8 & 0,86 \\
\hline 350 & & 18,8 & & 366 & 165 & 38 & 147 & 983 & 40 & 2,21 & 0 & 0,40 & 2,69 & 0,11 \\
\hline 358 & & & & & & & 170 & 646 & 14 & 2,13 & & & & 0,04 \\
\hline 374 & & & & & & & 23 & 78 & 62 & 2,19 & 1 & & & 0,17 \\
\hline 375 & & & & & 168 & 59 & 137 & 708 & 33 & 2,18 & 16 & & 1,93 & 0,09 \\
\hline 380 & & 26, & & & 161 & 111 & 189 & 593 & 293 & 2,23 & ,31 & 0,53 & 1,65 & 0,82 \\
\hline 384 & ( & & & & 16 & 16 & 130 & 610 & 11 & 2,18 & & & & 0,03 \\
\hline 402 & & & & & 16 & & 12 & 1396 & 0 & 2,20 & 9 & 0 & 3,98 & 0,00 \\
\hline 425 & & 21,8 & 27,1 & 355 & 164 & 39 & 113 & 867 & 0 & 2,17 & 0,11 & 0,32 & 2,44 & 0,00 \\
\hline 430 & schlecl & 20,1 & 12 & 369 & 16 & 62 & 143 & 660 & 40 & 2,21 & 0,17 & 0,39 & 1,79 & 0,11 \\
\hline 434 & & & & & 16 & & 91 & 845 & 60 & 2,18 & 6 & 0,25 & 2,35 & 0,17 \\
\hline 444 & & & & & 165 & 55 & 184 & 527 & 60 & 2,17 & & 0,51 & 1,47 & 0,17 \\
\hline 455 & lecht & 24,1 & 13,5 & 366 & 165 & 77 & 177 & 471 & 112 & 2,22 & 0,21 & 0,48 & 1,29 & 0,30 \\
\hline 456 & echt & 32,1 & 16,9 & 363 & 167 & 79 & 157 & 569 & 211 & 2,18 & 0,22 & 0,43 & 1,57 & 0,58 \\
\hline 458 & & 16 , & 27 & 3 & 16 & 43 & 12 & 895 & 71 & 2,18 & 0,12 & 0,35 & 2,49 & 0,20 \\
\hline 459 & schlecht & 20,4 & 13,7 & 364 & 168 & 45 & 144 & 640 & 13 & 2,16 & 0,12 & 0,40 & 1,76 & 0,04 \\
\hline
\end{tabular}

Ätzen: Masseverlust bei chemischer Probenaufreinigung durch Ätzen

Veraschen: Masseverlust beim Veraschen 
Tabelle 30: Kirchheim am Ries - Auswertungsrelevante Individualdaten für den Probensatz, der oberflächenbehandelt wurde.

\begin{tabular}{|c|c|c|c|c|c|c|c|c|c|c|c|c|}
\hline Grab & Histologie & $\begin{array}{c}\mathrm{Ca} \\
{[\mathrm{mg} / \mathrm{g}]}\end{array}$ & $\begin{array}{c}P \\
{[\mathrm{mg} / \mathrm{g}]}\end{array}$ & $\begin{array}{c}\mathrm{Ba} \\
{[\mu \mathrm{g} / \mathrm{g}]}\end{array}$ & $\begin{array}{c}\mathrm{Sr} \\
{[\mu \mathrm{g} / \mathrm{g}]}\end{array}$ & $\begin{array}{c}M g \\
{[\mu g / g]}\end{array}$ & $\begin{array}{c}\text { Al } \\
{[\mu \mathrm{g} / \mathrm{g}]}\end{array}$ & $\mathrm{Ca} / \mathrm{P}$ & $\begin{array}{l}\mathrm{Ba} / \mathrm{Ca} \\
{\left[\times 10^{-3}\right]}\end{array}$ & $\begin{array}{c}\mathrm{Sr} / \mathrm{Ca} \\
{\left[\times 10^{-3}\right]}\end{array}$ & $\begin{array}{l}\mathrm{Mg} / \mathrm{Ca} \\
{\left[\times 10^{-3}\right]}\end{array}$ & $\begin{array}{l}\mathrm{Al} / \mathrm{Ca} \\
{\left[\times 10^{-3}\right]}\end{array}$ \\
\hline 52 & gut & 372 & 163 & 37 & 129 & 1314 & 0 & 2,28 & 0,10 & 0,35 & 3,54 & 0,00 \\
\hline 73 & schlecht & 374 & 168 & 52 & 137 & 977 & 36 & 2,23 & 0,14 & 0,37 & 2,61 & 0,10 \\
\hline 73,1 & schlecht & 382 & 167 & 52 & 155 & 852 & 65 & 2,28 & 0,14 & 0,41 & 2,23 & 0,17 \\
\hline 74 & gut & 373 & 166 & 37 & 87 & 988 & 0 & 2,25 & 0,10 & 0,23 & 2,65 & 0,00 \\
\hline 81 & schlecht & 376 & 164 & 59 & 132 & 756 & 69 & 2,28 & 0,16 & 0,35 & 2,01 & 0,18 \\
\hline 84,1 & schlecht & 378 & 167 & 71 & 137 & 764 & 183 & 2,26 & 0,19 & 0,36 & 2,02 & 0,48 \\
\hline 92 & schlecht & 376 & 161 & 89 & 190 & 672 & 46 & 2,34 & 0,24 & 0,51 & 1,79 & 0,12 \\
\hline 106 & gut & 371 & 161 & 70 & 129 & 1287 & 48 & 2,30 & 0,19 & 0,35 & 3,46 & 0,13 \\
\hline 115 & gut & 373 & 162 & 30 & 117 & 995 & 69 & 2,29 & 0,08 & 0,31 & 2,67 & 0,18 \\
\hline 133,1 & schlecht & 374 & 166 & 76 & 153 & 854 & 299 & 2,24 & 0,20 & 0,41 & 2,29 & 0,80 \\
\hline 149 & gut & 371 & 163 & 34 & 121 & 1135 & 0 & 2,28 & 0,09 & 0,33 & 3,06 & 0,00 \\
\hline 152 & schlecht & 374 & 158 & 110 & 191 & 810 & 428 & 2,37 & 0,29 & 0,51 & 2,17 & 1,15 \\
\hline 159 & schlecht & 379 & 161 & 97 & 241 & 628 & 61 & 2,35 & 0,26 & 0,63 & 1,66 & 0,16 \\
\hline 166 & gut & 373 & 159 & 49 & 132 & 1706 & 54 & 2,35 & 0,13 & 0,35 & 4,57 & 0,14 \\
\hline 167 & schlecht & 383 & 163 & 68 & 179 & 667 & 35 & 2,34 & 0,18 & 0,47 & 1,74 & 0,09 \\
\hline 168 & gut & 373 & 162 & 49 & 147 & 1782 & 13 & 2,31 & 0,13 & 0,39 & 4,77 & 0,04 \\
\hline 172 & gut & 377 & 161 & 33 & 115 & 1518 & 0 & 2,34 & 0,09 & 0,30 & 4,03 & 0,00 \\
\hline 178 & gut & 374 & 161 & 51 & 149 & 1073 & 41 & 2,33 & 0,14 & 0,40 & 2,87 & 0,11 \\
\hline 185 & schlecht & 382 & 166 & 74 & 189 & 1110 & 124 & 2,30 & 0,19 & 0,50 & 2,90 & 0,32 \\
\hline 206 & schlecht & 385 & 166 & 70 & 169 & 686 & 0 & 2,32 & 0,18 & 0,44 & 1,78 & 0,00 \\
\hline 211 & schlecht & 386 & 166 & 69 & 175 & 814 & 351 & 2,33 & 0,18 & 0,45 & 2,11 & 0,91 \\
\hline 216 & gut & 382 & 162 & 50 & 124 & 1457 & 0 & 2,36 & 0,13 & 0,33 & 3,82 & 0,00 \\
\hline 233,1 & schlecht & 385 & 171 & 46 & 163 & 909 & 60 & 2,26 & 0,12 & 0,42 & 2,36 & 0,16 \\
\hline 235 & schlecht & 385 & 166 & 104 & 195 & 783 & 158 & 2,31 & 0,27 & 0,51 & 2,04 & 0,41 \\
\hline 248 & schlecht & 385 & 166 & 69 & 216 & 1027 & 285 & 2,32 & 0,18 & 0,56 & 2,67 & 0,74 \\
\hline 263 & schlecht & 385 & 164 & 87 & 174 & 815 & 42 & 2,35 & 0,23 & 0,45 & 2,12 & 0,11 \\
\hline 268 & gut & 378 & 166 & 40 & 119 & 1141 & 222 & 2,27 & 0,11 & 0,31 & 3,02 & 0,59 \\
\hline 298 & schlecht & 386 & 171 & 52 & 134 & 928 & 113 & 2,25 & 0,14 & 0,35 & 2,41 & 0,29 \\
\hline 304 & gut & 374 & 162 & 45 & 125 & 1200 & 0 & 2,31 & 0,12 & 0,33 & 3,20 & 0,00 \\
\hline 311,1 & schlecht & 377 & 161 & 119 & 236 & 816 & 317 & 2,35 & 0,31 & 0,62 & 2,16 & 0,84 \\
\hline 319,2 & schlecht & 385 & 172 & 41 & 133 & 933 & 276 & 2,24 & 0,11 & 0,35 & 2,42 & 0,72 \\
\hline 321,1 & gut & 380 & 163 & 36 & 142 & 1582 & 0 & 2,33 & 0,09 & 0,37 & 4,16 & 0,00 \\
\hline 345 & schlecht & 389 & 165 & 155 & 244 & 821 & 242 & 2,35 & 0,40 & 0,63 & 2,11 & 0,62 \\
\hline 350 & gut & 381 & 164 & 41 & 151 & 1136 & 30 & 2,33 & 0,11 & 0,40 & 2,99 & 0,08 \\
\hline 358 & schlecht & 386 & 172 & 61 & 185 & 742 & 0 & 2,25 & 0,16 & 0,48 & 1,92 & 0,00 \\
\hline 374 & schlecht & 383 & 167 & 52 & 244 & 892 & 17 & 2,30 & 0,14 & 0,64 & 2,33 & 0,04 \\
\hline 375 & schlecht & 383 & 164 & 64 & 149 & 802 & 27 & 2,33 & 0,17 & 0,39 & 2,09 & 0,07 \\
\hline 380 & schlecht & 382 & 159 & 112 & 201 & 716 & 188 & 2,40 & 0,29 & 0,53 & 1,87 & 0,49 \\
\hline 384 & schlecht & 383 & 163 & 55 & 151 & 693 & 11 & 2,35 & 0,14 & 0,40 & 1,81 & 0,03 \\
\hline 402 & gut & 379 & 163 & 36 & 136 & 1563 & 0 & 2,32 & 0,09 & 0,36 & 4,13 & 0,00 \\
\hline 425 & gut & 382 & 167 & 41 & 122 & 1006 & 0 & 2,28 & 0,11 & 0,32 & 2,63 & 0,00 \\
\hline 430 & schlecht & 369 & 159 & 68 & 156 & 738 & 16 & 2,32 & 0,18 & 0,42 & 2,00 & 0,04 \\
\hline 434 & gut & 371 & 163 & 22 & 100 & 959 & 32 & 2,28 & 0,06 & 0,27 & 2,59 & 0,09 \\
\hline 444 & schlecht & 372 & 159 & 62 & 199 & 638 & 27 & 2,34 & 0,17 & 0,53 & 1,72 & 0,07 \\
\hline 455 & schlecht & 374 & 159 & 82 & 190 & 572 & 93 & 2,36 & 0,22 & 0,51 & 1,53 & 0,25 \\
\hline 456 & schlecht & 368 & 157 & 87 & 178 & 732 & 178 & 2,34 & 0,24 & 0,48 & 1,99 & 0,48 \\
\hline 458 & gut & 365 & 160 & 46 & 137 & 1019 & 39 & 2,28 & 0,13 & 0,37 & 2,79 & 0,11 \\
\hline 459 & schlecht & 369 & 163 & 48 & 152 & 762 & 0 & 2,27 & 0,13 & 0,41 & 2,07 & 0,00 \\
\hline
\end{tabular}


Tabelle 31: Kirchheim am Ries - Auswertungsrelevante Individualdaten für den Probensatz, der oberflächenbehandelt und geätzt wurde.

\begin{tabular}{|c|c|c|c|c|c|c|c|c|c|c|c|c|}
\hline Grab & Histologie & $\begin{array}{c}\mathrm{Ca} \\
{[\mathrm{mg} / \mathrm{g}]}\end{array}$ & $\begin{array}{c}P \\
{[\mathrm{mg} / \mathrm{g}]}\end{array}$ & $\begin{array}{c}\mathrm{Ba} \\
{[\mu \mathrm{g} / \mathrm{g}]}\end{array}$ & $\begin{array}{c}\mathrm{Sr} \\
{[\mu \mathrm{g} / \mathrm{g}]}\end{array}$ & $\begin{array}{c}M g \\
{[\mu g / g]}\end{array}$ & $\begin{array}{c}\text { Al } \\
{[\mu \mathrm{g} / \mathrm{g}]}\end{array}$ & $\mathrm{Ca} / \mathrm{P}$ & $\begin{array}{l}\mathrm{Ba} / \mathrm{Ca} \\
{\left[\times 10^{-3}\right]}\end{array}$ & $\begin{array}{c}\mathrm{Sr} / \mathrm{Ca} \\
{\left[\times 10^{-3}\right]}\end{array}$ & $\begin{array}{l}\mathrm{Mg} / \mathrm{Ca} \\
{\left[\times 10^{-3}\right]}\end{array}$ & $\begin{array}{l}\mathrm{Al} / \mathrm{Ca} \\
{\left[\times 10^{-3}\right]}\end{array}$ \\
\hline 52 & gut & 370 & 168 & 36 & 126 & 1346 & 0 & 2,20 & 0,10 & 0,34 & 3,63 & 0,00 \\
\hline 73 & schlecht & 362 & 168 & 49 & 124 & 901 & 61 & 2,16 & 0,14 & 0,34 & 2,49 & 0,17 \\
\hline 73,1 & schlecht & 366 & 170 & 47 & 137 & 671 & 54 & 2,16 & 0,13 & 0,38 & 1,83 & 0,15 \\
\hline 74 & gut & 358 & 165 & 38 & 83 & 912 & 0 & 2,17 & 0,11 & 0,23 & 2,55 & 0,00 \\
\hline 81 & schlecht & 362 & 168 & 51 & 118 & 636 & 54 & 2,16 & 0,14 & 0,33 & 1,76 & 0,15 \\
\hline 84,1 & schlecht & 378 & 168 & 71 & 132 & 643 & 255 & 2,25 & 0,19 & 0,35 & 1,70 & 0,67 \\
\hline 92 & schlecht & 378 & 163 & 89 & 182 & 611 & 75 & 2,32 & 0,23 & 0,48 & 1,62 & 0,20 \\
\hline 106 & gut & 372 & 162 & 66 & 122 & 1147 & 37 & 2,30 & 0,18 & 0,33 & 3,08 & 0,10 \\
\hline 115 & gut & 372 & 163 & 28 & 110 & 970 & 41 & 2,27 & 0,08 & 0,30 & 2,61 & 0,11 \\
\hline 133,1 & schlecht & 373 & 173 & 54 & 130 & 653 & 281 & 2,16 & 0,14 & 0,35 & 1,75 & 0,75 \\
\hline 149 & gut & 372 & 159 & 32 & 117 & 1135 & 0 & 2,34 & 0,09 & 0,31 & 3,05 & 0,00 \\
\hline 152 & schlecht & 376 & 167 & 107 & 176 & 655 & 465 & 2,25 & 0,28 & 0,47 & 1,74 & 1,24 \\
\hline 159 & schlecht & 381 & 165 & 94 & 227 & 572 & 89 & 2,31 & 0,25 & 0,59 & 1,50 & 0,23 \\
\hline 166 & gut & 371 & 158 & 50 & 128 & 1584 & 61 & 2,35 & 0,14 & 0,34 & 4,27 & 0,16 \\
\hline 167 & schlecht & 379 & 167 & 59 & 157 & 572 & 49 & 2,27 & 0,16 & 0,41 & 1,51 & 0,13 \\
\hline 168 & gut & 372 & 163 & 47 & 136 & 1788 & 22 & 2,28 & 0,13 & 0,37 & 4,81 & 0,06 \\
\hline 172 & gut & 367 & 158 & 28 & 107 & 1596 & 0 & 2,33 & 0,08 & 0,29 & 4,34 & 0,00 \\
\hline 178 & gut & 375 & 165 & 48 & 144 & 989 & 35 & 2,27 & 0,13 & 0,38 & 2,64 & 0,09 \\
\hline 185 & schlecht & 377 & 166 & 74 & 183 & 977 & 215 & 2,27 & 0,20 & 0,48 & 2,59 & 0,57 \\
\hline 206 & schlecht & 380 & 166 & 65 & 155 & 630 & 0 & 2,29 & 0,17 & 0,41 & 1,66 & 0,00 \\
\hline 211 & schlecht & 381 & 171 & 68 & 161 & 654 & 406 & 2,23 & 0,18 & 0,42 & 1,72 & 1,07 \\
\hline 216 & gut & 370 & 158 & 45 & 110 & 1310 & 0 & 2,34 & 0,12 & 0,30 & 3,54 & 0,00 \\
\hline 233,1 & schlecht & 376 & 168 & 46 & 157 & 771 & 58 & 2,24 & 0,12 & 0,42 & 2,05 & 0,15 \\
\hline 235 & schlecht & 380 & 169 & 64 & 146 & 619 & 95 & 2,24 & 0,17 & 0,39 & 1,63 & 0,25 \\
\hline 248 & schlecht & 381 & 168 & 56 & 198 & 839 & 245 & 2,26 & 0,15 & 0,52 & 2,20 & 0,64 \\
\hline 263 & schlecht & 380 & 166 & 82 & 158 & 689 & 74 & 2,30 & 0,22 & 0,41 & 1,81 & 0,19 \\
\hline 268 & gut & 374 & 167 & 34 & 106 & 1036 & 241 & 2,24 & 0,09 & 0,28 & 2,77 & 0,65 \\
\hline 298 & schlecht & 380 & 170 & 41 & 115 & 841 & 144 & 2,23 & 0,11 & 0,30 & 2,22 & 0,38 \\
\hline 304 & gut & 377 & 163 & 41 & 116 & 1169 & 0 & 2,31 & 0,11 & 0,31 & 3,10 & 0,00 \\
\hline 311,1 & schlecht & 379 & 167 & 115 & 222 & 694 & 419 & 2,27 & 0,30 & 0,59 & 1,83 & 1,11 \\
\hline 319,2 & schlecht & 379 & 174 & 38 & 119 & 767 & 414 & 2,18 & 0,10 & 0,31 & 2,02 & 1,09 \\
\hline 321,1 & out & 375 & 160 & 31 & 139 & 1432 & 0 & 2,34 & 0,08 & 0,37 & 3,82 & 0,00 \\
\hline 345 & schlecht & 379 & 164 & 137 & 220 & 694 & 248 & 2,30 & 0,36 & 0,58 & 1,83 & 0,65 \\
\hline 350 & gut & 374 & 163 & 39 & 143 & 1094 & 17 & 2,29 & 0,10 & 0,38 & 2,92 & 0,05 \\
\hline 358 & schlecht & 380 & 171 & 54 & 174 & 612 & 0 & 2,22 & 0,14 & 0,46 & 1,61 & 0,00 \\
\hline 374 & schlecht & 387 & 172 & 50 & 244 & 843 & 37 & 2,26 & 0,13 & 0,63 & 2,18 & 0,10 \\
\hline 375 & schlecht & 384 & 171 & 52 & 127 & 708 & 30 & 2,24 & 0,13 & 0,33 & 1,85 & 0,08 \\
\hline 380 & schlecht & 383 & 168 & 103 & 187 & 572 & 208 & 2,29 & 0,27 & 0,49 & 1,49 & 0,54 \\
\hline 384 & schlecht & 383 & 168 & 52 & 140 & 651 & 0 & 2,28 & 0,14 & 0,37 & 1,70 & 0,00 \\
\hline 402 & gut & 377 & 166 & 29 & 126 & 1520 & 10 & 2,28 & 0,08 & 0,33 & 4,03 & 0,03 \\
\hline 425 & gut & 379 & 169 & 42 & 114 & 945 & 13 & 2,24 & 0,11 & 0,30 & 2,50 & 0,03 \\
\hline 430 & schlecht & 384 & 170 & 64 & 146 & 642 & 30 & 2,26 & 0,17 & 0,38 & 1,67 & 0,08 \\
\hline 434 & at & 381 & 169 & 22 & 95 & 941 & 23 & 2,25 & 0,06 & 0,25 & 2,47 & 0,06 \\
\hline 444 & schlecht & 379 & 168 & 54 & 186 & 584 & 33 & 2,26 & 0,14 & 0,49 & 1,54 & 0,09 \\
\hline 455 & schlecht & 381 & 166 & 75 & 176 & 482 & 76 & 2,30 & 0,20 & 0,46 & 1,26 & 0,20 \\
\hline 456 & schlecht & 382 & 170 & 82 & 163 & 595 & 185 & 2,24 & 0,21 & 0,43 & 1,56 & 0,49 \\
\hline 458 & gut & 378 & 166 & 44 & 130 & 971 & 28 & 2,27 & 0,12 & 0,34 & 2,57 & 0,07 \\
\hline 459 & schlecht & 380 & 169 & 47 & 147 & 666 & 0 & 2,25 & 0,12 & 0,39 & 1,75 & 0,00 \\
\hline
\end{tabular}

Tabelle 32: Kirchheim am Ries - Daten von den Natriumanalysen

\begin{tabular}{|l|c|c|c|c|}
\hline Grab & Histologie & $\begin{array}{c}\mathbf{C a} \\
{[\mathbf{m g} / \mathbf{g}]}\end{array}$ & $\begin{array}{c}\mathbf{N a} \\
{[\mathbf{\mu} / \mathbf{g}]}\end{array}$ & $\begin{array}{c}\mathbf{N a} / \mathbf{C a} \\
{\left[\times \mathbf{1 0}^{-3}\right]}\end{array}$ \\
\hline 159 & schlecht & 384 & 3101 & 8,09 \\
166 & gut & 377 & 6550 & 17,36 \\
167 & schlecht & 384 & 2888 & 7,51 \\
168 & gut & 378 & 6100 & 16,13 \\
172 & gut & 374 & 6427 & 17,17 \\
321,1 & gut & 377 & 6686 & 17,72 \\
380 & schlecht & 383 & 2882 & 7,53 \\
402 & gut & 378 & 6442 & 17,05 \\
444 & schlecht & 383 & 3059 & 7,99 \\
455 & schlecht & 385 & 2520 & 6,55 \\
\hline
\end{tabular}


Tabelle 33: Datenliste der EPMA-Einzelpunktmessungen

\begin{tabular}{|c|c|c|c|c|c|c|c|c|c|c|}
\hline Meßpunkt & $\begin{array}{l}\text { Mineral- } \\
\text { zustand }\end{array}$ & $\begin{array}{c}\mathrm{Ca} \\
{[\mathrm{mg} / \mathrm{g}]}\end{array}$ & $\begin{array}{c}P \\
{[\mathrm{mg} / \mathrm{g}]}\end{array}$ & $\begin{array}{c}\mathrm{Mg} \\
{[\mu / g]}\end{array}$ & $\begin{array}{c}\mathrm{Na} \\
{[\mu / g]}\end{array}$ & $\begin{array}{c}S \\
{[\mu / g]}\end{array}$ & $\mathrm{Ca} / \mathrm{P}$ & $\begin{array}{l}\mathrm{Mg} / \mathrm{Ca} \\
{\left[\times 10^{-3}\right]}\end{array}$ & $\begin{array}{l}\mathrm{Na} / \mathrm{Ca} \\
{\left[\times 10^{-3}\right]}\end{array}$ & $\begin{array}{l}\mathrm{S} / \mathrm{Ca} \\
{\left[\times 10^{-3}\right]}\end{array}$ \\
\hline 1 & "nativ" & 292 & 121 & 687 & 3019 & 1206 & 2,41 & 2,35 & 10,33 & 4,13 \\
\hline 3 & "nativ" & 277 & 117 & 585 & 2715 & 1450 & 2,36 & 2,11 & 9,81 & 5,24 \\
\hline 4 & "nativ" & 285 & 124 & 663 & 2975 & 1330 & 2,30 & 2,33 & 10,43 & 4,66 \\
\hline 5 & "nativ" & 290 & 126 & 911 & 3064 & 1250 & 2,30 & 3,14 & 10,55 & 4,30 \\
\hline 7 & verändert & 328 & 140 & 1248 & 430 & 1093 & 2,35 & 3,80 & 1,31 & 3,33 \\
\hline 8 & "nativ" & 300 & 131 & 742 & 3346 & 969 & 2,29 & 2,48 & 11,17 & 3,24 \\
\hline 9 & "nativ" & 288 & 126 & 585 & 2693 & 1161 & 2,28 & 2,03 & 9,36 & 4,04 \\
\hline 10 & verändert & 328 & 144 & 603 & 786 & 637 & 2,27 & 1,84 & 2,39 & 1,94 \\
\hline 11 & "nativ" & 298 & 132 & 814 & 3116 & 1101 & 2,25 & 2,74 & 10,47 & 3,70 \\
\hline 12 & verändert & 344 & 152 & 235 & 0 & 713 & 2,26 & 0,68 & 0,00 & 2,07 \\
\hline 17 & "nativ" & 312 & 141 & 868 & 3583 & 1069 & 2,21 & 2,79 & 11,49 & 3,43 \\
\hline 18 & verändert & 354 & 162 & 350 & 0 & 701 & 2,19 & 0,99 & 0,00 & 1,98 \\
\hline 19 & "nativ" & 293 & 126 & 712 & 3190 & 961 & 2,32 & 2,43 & 10,89 & 3,28 \\
\hline 20 & "nativ" & 288 & 128 & 868 & 3257 & 1278 & 2,25 & 3,02 & 11,32 & 4,44 \\
\hline 21 & "nativ" & 300 & 131 & 947 & 3561 & 949 & 2,28 & 3,16 & 11,88 & 3,17 \\
\hline 22 & verändert & 362 & 162 & 470 & 675 & 621 & 2,24 & 1,30 & 1,86 & 1,71 \\
\hline 23 & verändert & 188 & 86 & 0 & 0 & 332 & 2,19 & 0,00 & 0,00 & 1,76 \\
\hline 24 & "nativ" & 311 & 140 & 850 & 2737 & 1121 & 2,22 & 2,73 & 8,80 & 3,61 \\
\hline 25 & verändert & 365 & 167 & 543 & 1254 & 501 & 2,18 & 1,49 & 3,43 & 1,37 \\
\hline 26 & verändert & 362 & 168 & 519 & 1921 & 497 & 2,15 & 1,43 & 5,30 & 1,37 \\
\hline 27 & verändert & 369 & 165 & 651 & 1625 & 405 & 2,24 & 1,77 & 4,41 & 1,10 \\
\hline 36 & verändert & 207 & 90 & 199 & 1046 & 224 & 2,29 & 0,96 & 5,06 & 1,08 \\
\hline 37 & verändert & 311 & 137 & 736 & 1736 & 1129 & 2,27 & 2,37 & 5,58 & 3,63 \\
\hline 38 & verändert & 377 & 170 & 549 & 1113 & 589 & 2,21 & 1,45 & 2,95 & 1,56 \\
\hline 39 & verändert & 351 & 157 & 356 & 712 & 669 & 2,24 & 1,01 & 2,03 & 1,91 \\
\hline 40 & "nativ" & 270 & 118 & 905 & 3301 & 1025 & 2,30 & 3,35 & 12,23 & 3,80 \\
\hline 41 & "nativ" & 286 & 130 & 1019 & 3064 & 941 & 2,20 & 3,57 & 10,72 & 3,29 \\
\hline 42 & "nativ" & 295 & 129 & 766 & 2255 & 1105 & 2,29 & 2,60 & 7,66 & 3,75 \\
\hline 43 & "nativ" & 288 & 128 & 911 & 3494 & 1214 & 2,24 & 3,16 & 12,14 & 4,22 \\
\hline 44 & verändert & 362 & 158 & 271 & 0 & 853 & 2,29 & 0,75 & 0,00 & 2,35 \\
\hline 45 & "nativ" & 337 & 151 & 609 & 1254 & 541 & 2,23 & 1,81 & 3,72 & 1,60 \\
\hline 46 & verändert & 217 & 96 & 374 & 0 & 749 & 2,27 & 1,72 & 0,00 & 3,44 \\
\hline 47 & "nativ" & 286 & 124 & 868 & 2864 & 1129 & 2,31 & 3,03 & 10,00 & 3,94 \\
\hline 48 & "nativ" & 278 & 124 & 959 & 2767 & 1306 & 2,25 & 3,45 & 9,96 & 4,70 \\
\hline 49 & "nativ" & 297 & 132 & 742 & 2559 & 1430 & 2,24 & 2,50 & 8,63 & 4,82 \\
\hline 50 & "nativ" & 295 & 130 & 868 & 3628 & 1129 & 2,28 & 2,94 & 12,30 & 3,83 \\
\hline 51 & "nativ" & 291 & 130 & 850 & 3235 & 1129 & 2,24 & 2,92 & 11,11 & 3,88 \\
\hline 52 & "nativ" & 278 & 125 & 1061 & 3576 & 1186 & 2,23 & 3,82 & 12,86 & 4,26 \\
\hline 53 & "nativ" & 299 & 135 & 639 & 2196 & 1306 & 2,22 & 2,14 & 7,34 & 4,36 \\
\hline 54 & "nativ" & 279 & 124 & 1049 & 2982 & 1218 & 2,24 & 3,76 & 10,69 & 4,36 \\
\hline 55 & "nativ" & 283 & 126 & 1073 & 3561 & 1198 & 2,26 & 3,79 & 12,58 & 4,23 \\
\hline 56 & "nativ" & 282 & 132 & 808 & 2567 & 1330 & 2,13 & 2,87 & 9,10 & 4,71 \\
\hline 57 & "nativ" & 290 & 132 & 850 & 3479 & 1097 & 2,20 & 2,93 & 12,00 & 3,79 \\
\hline 58 & "nativ" & 299 & 134 & 712 & 2760 & 1278 & 2,23 & 2,38 & 9,23 & 4,27 \\
\hline
\end{tabular}


Fortsetzung Tabelle 33

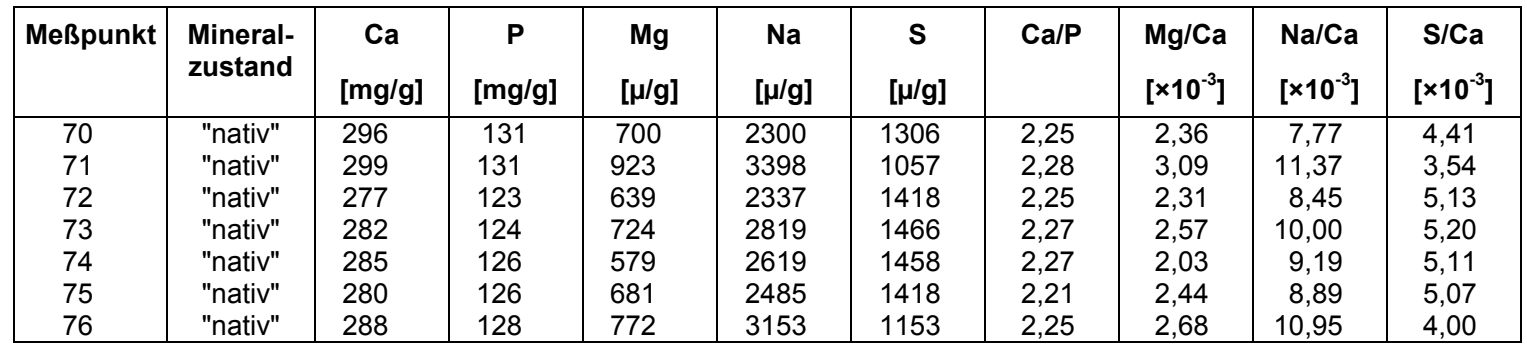

Tabelle 34: Datenliste der LA-ICP-TOFMS Analysen - Meßsignale und Signalquotienten.

\begin{tabular}{|c|c|c|c|c|c|c|c|}
\hline Meßpunkt & $\begin{array}{c}\mathrm{Ca} \\
\text { [mv] }\end{array}$ & $\begin{array}{l}{ }^{137} \mathrm{Ba} \\
\text { [cps] }\end{array}$ & $\begin{array}{l}{ }^{88} \mathrm{Sr} \\
{[\mathrm{mV}]}\end{array}$ & $\begin{array}{l}{ }^{238} \mathrm{U} \\
{[\mathrm{cps}]}\end{array}$ & $\mathrm{Ba} / \mathrm{Ca}$ & $\mathrm{Sr} / \mathrm{Ca}$ & $\mathrm{U} / \mathrm{Ca}$ \\
\hline 1 & 949 & 2764 & 45 & 773 & 2,911 & 0,048 & 0,814 \\
\hline 2 & 923 & 1824 & 37 & 810 & 1,976 & 0,040 & 0,878 \\
\hline 3 & 1229 & 3338 & 54 & 1125 & 2,717 & 0,044 & 0,916 \\
\hline 4 & 1153 & 2489 & 43 & 1045 & 2,158 & 0,037 & 0,906 \\
\hline 5 & 1421 & 4360 & 55 & 964 & 3,068 & 0,039 & 0,678 \\
\hline 6 & 1161 & 2800 & 42 & 631 & 2,411 & 0,037 & 0,543 \\
\hline 7 & 1562 & 4044 & 70 & 635 & 2,590 & 0,045 & 0,406 \\
\hline 8 & 1545 & 4488 & 70 & 408 & 2,905 & 0,045 & 0,264 \\
\hline 9 & 1324 & 2655 & 51 & 213 & 2,005 & 0,039 & 0,161 \\
\hline 10 & 1339 & 2998 & 54 & 89 & 2,239 & 0,041 & 0,067 \\
\hline 11 & 1644 & 3147 & 67 & 1 & 1,914 & 0,041 & 0,000 \\
\hline 12 & 1234 & 2275 & 50 & 37 & 1,843 & 0,041 & 0,030 \\
\hline 13 & 1468 & 2287 & 54 & 218 & 1,558 & 0,037 & 0,148 \\
\hline 14 & 1534 & 2681 & 62 & -4 & 1,748 & 0,041 & 0,000 \\
\hline 15 & 1450 & 2157 & 49 & 9 & 1,488 & 0,034 & 0,000 \\
\hline 16 & 1209 & 1717 & 40 & 39 & 1,420 & 0,033 & 0,032 \\
\hline 17 & 1210 & 1668 & 38 & -30 & 1,378 & 0,031 & 0,000 \\
\hline 18 & 1384 & 1973 & 47 & -29 & 1,425 & 0,034 & 0,000 \\
\hline 19 & 1074 & 920 & 30 & 15 & 0,856 & 0,028 & 0,014 \\
\hline 20 & 1498 & 2172 & 48 & 89 & 1,450 & 0,032 & 0,060 \\
\hline 21 & 1376 & 2340 & 47 & 223 & 1,701 & 0,034 & 0,162 \\
\hline 22 & 1506 & 3052 & 61 & 81 & 2,026 & 0,041 & 0,054 \\
\hline 23 & 1310 & 2585 & 53 & 273 & 1,974 & 0,040 & 0,209 \\
\hline 24 & 1294 & 2109 & 48 & -64 & 1,630 & 0,037 & 0,000 \\
\hline 25 & 1266 & 1293 & 38 & 114 & 1,021 & 0,030 & 0,090 \\
\hline 26 & 1313 & 1491 & 42 & 79 & 1,136 & 0,032 & 0,060 \\
\hline 27 & 1515 & 1814 & 44 & 41 & 1,198 & 0,029 & 0,027 \\
\hline 28 & 1431 & 1585 & 38 & 241 & 1,108 & 0,027 & 0,168 \\
\hline 29 & 1153 & 1200 & 31 & 209 & 1,041 & 0,027 & 0,181 \\
\hline Harz & 59 & 870 & 6 & -13 & & & \\
\hline
\end{tabular}


Tabelle 35: Rezente Femurdiaphyse - Liste der Einzelproben einschließlich auswertungsrelevanter Daten.

\begin{tabular}{|c|c|c|c|c|c|c|c|c|c|c|}
\hline Probe & Höhe & Bereich & Veraschen & $\begin{array}{c}\mathrm{Ca} \\
{[\mathrm{mg} / \mathrm{g}]}\end{array}$ & $\begin{array}{c}P \\
{[\mathrm{mg} / \mathrm{g}]}\end{array}$ & $\begin{array}{c}\mathrm{Sr} \\
{[\mu / g]}\end{array}$ & $\begin{array}{c}\mathbf{M g} \\
{[\mu / g]}\end{array}$ & $\mathrm{Ca} / \mathrm{P}$ & $\begin{array}{c}\mathrm{Sr} / \mathrm{Ca} \\
{\left[\times 10^{-3}\right]}\end{array}$ & $\begin{array}{l}\mathrm{Mg} / \mathrm{Ca} \\
{\left[\times 10^{-3}\right]}\end{array}$ \\
\hline 1 & 1 & 1 & 29,9 & 364 & 165 & 207 & 4265 & 2,21 & 0,57 & 11,72 \\
\hline 2 & 1 & 2 & 30,4 & 357 & 160 & 181 & 3974 & 2,22 & 0,51 & 11,14 \\
\hline 3 & 1 & 3 & 30,1 & 358 & 162 & 187 & 4072 & 2,21 & 0,52 & 11,38 \\
\hline 4 & 1 & 4 & 29,7 & 362 & 164 & 207 & 4228 & 2,20 & 0,57 & 11,70 \\
\hline 5 & 1 & 5 & 30,1 & 360 & 164 & 213 & 4216 & 2,20 & 0,59 & 11,72 \\
\hline 6 & 1 & 6 & 29,3 & 362 & 165 & 181 & 4230 & 2,20 & 0,50 & 11,70 \\
\hline 7 & 1 & 7 & 29,5 & 356 & 162 & 196 & 4155 & 2,20 & 0,55 & 11,66 \\
\hline 8 & 1 & 8 & 29,5 & 358 & 163 & 198 & 4230 & 2,20 & 0,55 & 11,82 \\
\hline 9 & 2 & 1 & 29,7 & 357 & 162 & 206 & 4260 & 2,20 & 0,58 & 11,93 \\
\hline 10 & 2 & 2 & 30,3 & 356 & 161 & 183 & 3969 & 2,22 & 0,52 & 11,15 \\
\hline 11 & 2 & 3 & 30,0 & 357 & 162 & 190 & 4075 & 2,20 & 0,53 & 11,40 \\
\hline 12 & 2 & 4 & 29,6 & 350 & 159 & 204 & 4095 & 2,19 & 0,58 & 11,72 \\
\hline 13 & 2 & 5 & 29,5 & 353 & 161 & 221 & 4221 & 2,19 & 0,63 & 11,96 \\
\hline 14 & 2 & 6 & 29,2 & 356 & 163 & 191 & 4177 & 2,18 & 0,54 & 11,74 \\
\hline 15 & 2 & 7 & 29,3 & 354 & 161 & 180 & 4145 & 2,19 & 0,51 & 11,71 \\
\hline 16 & 2 & 8 & 29,3 & 355 & 162 & 190 & 4224 & 2,19 & 0,54 & 11,88 \\
\hline 17 & 3 & 1 & 29,5 & 353 & 161 & 201 & 4265 & 2,19 & 0,57 & 12,10 \\
\hline 18 & 3 & 2 & 30,2 & 354 & 161 & 179 & 3951 & 2,20 & 0,50 & 11,17 \\
\hline 19 & 3 & 3 & 30,1 & 345 & 157 & 184 & 3930 & 2,19 & 0,53 & 11,38 \\
\hline 20 & 3 & 4 & 30,4 & 349 & 159 & 201 & 4078 & 2,20 & 0,58 & 11,68 \\
\hline 21 & 3 & 5 & 29,5 & 346 & 159 & 213 & 4118 & 2,18 & 0,61 & 11,90 \\
\hline 22 & 3 & 6 & 29,3 & 352 & 161 & 198 & 4130 & 2,18 & 0,56 & 11,74 \\
\hline 23 & 3 & 7 & 29,4 & 349 & 159 & 174 & 4063 & 2,19 & 0,50 & 11,65 \\
\hline 24 & 3 & 8 & 29,5 & 349 & 160 & 181 & 4144 & 2,18 & 0,52 & 11,88 \\
\hline 25 & 4 & 1 & 29,3 & 351 & 161 & 190 & 4217 & 2,18 & 0,54 & 12,03 \\
\hline 26 & 4 & 2 & 30,2 & 350 & 160 & 179 & 3941 & 2,19 & 0,51 & 11,25 \\
\hline 27 & 4 & 3 & 30,2 & 351 & 160 & 181 & 3943 & 2,19 & 0,52 & 11,23 \\
\hline 28 & 4 & 4 & 30,1 & 350 & 161 & 198 & 4108 & 2,18 & 0,57 & 11,73 \\
\hline 29 & 4 & 5 & 30,2 & 349 & 160 & 212 & 4139 & 2,18 & 0,61 & 11,87 \\
\hline 30 & 4 & 6 & 29,5 & 348 & 160 & 203 & 4077 & 2,18 & 0,58 & 11,72 \\
\hline 31 & 4 & 7 & 29,4 & 356 & 163 & 181 & 4132 & 2,18 & 0,51 & 11,62 \\
\hline 32 & 4 & 8 & 29,5 & 352 & 161 & 179 & 4160 & 2,18 & 0,51 & 11,81 \\
\hline 33 & 5 & 1 & 29,3 & 344 & 159 & 175 & 4105 & 2,17 & 0,51 & 11,93 \\
\hline 34 & 5 & 2 & 29,9 & 346 & 159 & 183 & 3936 & 2,18 & 0,53 & 11,37 \\
\hline 35 & 5 & 3 & 30,1 & 352 & 161 & 184 & 3907 & 2,18 & 0,52 & 11,11 \\
\hline 36 & 5 & 4 & 30,7 & 350 & 160 & 188 & 3981 & 2,18 & 0,54 & 11,37 \\
\hline 37 & 5 & 5 & 30,0 & 350 & 162 & 204 & 4144 & 2,17 & 0,58 & 11,83 \\
\hline 38 & 5 & 6 & 29,6 & 349 & 161 & 202 & 4093 & 2,17 & 0,58 & 11,72 \\
\hline 39 & 5 & 7 & 29,5 & 351 & 161 & 187 & 4044 & 2,17 & 0,53 & 11,53 \\
\hline 40 & 5 & 8 & 29,5 & 348 & 160 & 173 & 4110 & 2,18 & 0,50 & 11,80 \\
\hline
\end{tabular}

Tabelle 36: Rezente Femurdiaphyse - Natriumanalysen

\begin{tabular}{|c|c|c|c|c|c|}
\hline Probe & Höhe & Bereich & $\mathbf{C a}$ & $\mathbf{N a}$ & $\mathbf{N a / C a}$ \\
\hline & & & {$[\mathbf{m g} / \mathbf{g}]$} & {$[\boldsymbol{\mu} / \mathbf{g}]$} & {$\left[\times \mathbf{1 0}^{-3}\right]$} \\
\hline 17 & 3 & 1 & 371 & 11983 & 32,27 \\
18 & 3 & 2 & 395 & 12884 & 32,63 \\
19 & 3 & 3 & 364 & 11756 & 32,32 \\
20 & 3 & 4 & 366 & 11806 & 32,28 \\
21 & 3 & 5 & 367 & 11808 & 32,18 \\
22 & 3 & 6 & 368 & 11827 & 32,16 \\
23 & 3 & 7 & 363 & 11583 & 31,91 \\
24 & 3 & 8 & 362 & 11669 & 32,21 \\
\hline
\end{tabular}


Tabelle 37: Weingarten - Statistisch deskriptive Daten für die drei Gruppierungsfaktoren

\begin{tabular}{|c|c|c|c|c|c|c|c|c|c|c|}
\hline \multirow[t]{2}{*}{ Gruppe } & \multirow[t]{2}{*}{ Variable } & \multirow[t]{2}{*}{ Einheit } & \multirow[t]{2}{*}{ Anzahl } & \multirow[t]{2}{*}{$\begin{array}{l}\text { Mittel- } \\
\text { wert }\end{array}$} & \multirow[t]{2}{*}{ Median } & \multirow[t]{2}{*}{ Min } & \multirow[t]{2}{*}{ Max } & \multirow[t]{2}{*}{$\begin{array}{c}\text { Standard- } \\
\text { abweichung }\end{array}$} & \multicolumn{2}{|c|}{$\begin{array}{l}\text { Normalverteilungstest } \\
\text { Shapiro-Wilk's W-Test }\end{array}$} \\
\hline & & & & & & & & & $\mathbf{w}$ & $p$ \\
\hline \multirow{5}{*}{$\begin{array}{l}\text { Histologie } \\
\text { gut }\end{array}$} & Ätzen & [\%] & 25 & 11,5 & 10,3 & 5,6 & 39,3 & 6,6 & 0,63 & 0,0000 \\
\hline & Veraschen & [\%] & 24 & 29,2 & 29,2 & 22,5 & 33,7 & 2,4 & 0,84 & 0,0010 \\
\hline & $\mathrm{Ca} / \mathrm{P}$ & & 35 & 2,22 & 2,24 & 2,06 & 2,37 & 0,09 & 0,95 & 0,1223 \\
\hline & $\mathrm{Sr} / \mathrm{Ca}$ & {$\left[\times 10^{-3}\right]$} & 56 & 0,88 & 0,84 & 0,66 & 1,60 & 0,17 & 0,77 & 0,0000 \\
\hline & $\mathrm{Ba} / \mathrm{Ca}$ & {$\left[\times 10^{-3}\right]$} & 56 & 0,23 & 0,23 & 0,10 & 0,43 & 0,06 & 0,96 & 0,1203 \\
\hline \multirow{5}{*}{$\begin{array}{l}\text { Histologie } \\
\text { schlecht }\end{array}$} & Ätzen & & 38 & 20,1 & 19,0 & 6,2 & 46,00 & 8,0 & 0,95 & 0,1466 \\
\hline & Veraschen & & 28 & 20,2 & 18,8 & 11,7 & 31,00 & 5,2 & 0,95 & 0,2047 \\
\hline & $\mathrm{Ca} / \mathrm{P}$ & & 100 & 2,17 & 2,17 & 1,87 & 2,50 & 0,12 & 0,99 & 0,9424 \\
\hline & $\mathrm{Sr} / \mathrm{Ca}$ & {$\left[\times 10^{-3}\right]$} & 122 & 1,40 & 1,41 & 0,73 & 1,83 & 0,19 & 0,98 & 0,3235 \\
\hline & $\mathrm{Ba} / \mathrm{Ca}$ & {$\left[\times 10^{-3}\right]$} & 122 & 0,30 & 0,30 & 0,11 & 0,43 & 0,06 & 0,98 & 0,3937 \\
\hline \multirow{2}{*}{$\begin{array}{l}\text { niederer } \\
\text { Rang }\end{array}$} & $\mathrm{Sr} / \mathrm{Ca}$ & {$\left[\times 10^{-3}\right]$} & 95 & 1,31 & 1,37 & 0,71 & 1,79 & 0,27 & 0,92 & 0,0000 \\
\hline & $\mathrm{Ba} / \mathrm{Ca}$ & {$\left[\times 10^{-3}\right]$} & 95 & 0,29 & 0,29 & 0,10 & 0,43 & 0,07 & 0,98 & 0,3956 \\
\hline \multirow{2}{*}{$\begin{array}{l}\text { hoher } \\
\text { Rang }\end{array}$} & $\mathrm{Sr} / \mathrm{Ca}$ & {$\left[\times 10^{-3}\right]$} & 83 & 1,15 & 1,11 & 0,66 & 1,83 & 0,32 & 0,90 & 0,0000 \\
\hline & $\mathrm{Ba} / \mathrm{Ca}$ & {$\left[\times 10^{-3}\right]$} & 83 & 0,27 & 0,26 & 0,11 & 0,43 & 0,07 & 0,98 & 0,4704 \\
\hline \multirow[t]{2}{*}{ männlich } & $\mathrm{Sr} / \mathrm{Ca}$ & {$\left[\times 10^{-3}\right]$} & 78 & 1,21 & 1,31 & 0,66 & 1,83 & 0,30 & 0,91 & 0,0000 \\
\hline & $\mathrm{Ba} / \mathrm{Ca}$ & {$\left[\times 10^{-3}\right]$} & 78 & 0,28 & 0,28 & 0,11 & 0,43 & 0,07 & 0,97 & 0,3104 \\
\hline \multirow[t]{2}{*}{ weiblich } & $\mathrm{Sr} / \mathrm{Ca}$ & {$\left[\times 10^{-3}\right]$} & 100 & 1,26 & 1,31 & 0,68 & 1,79 & 0,31 & 0,92 & 0,0000 \\
\hline & $\mathrm{Ba} / \mathrm{Ca}$ & {$\left[\times 10^{-3}\right]$} & 100 & 0,28 & 0,28 & 0,10 & 0,41 & 0.07 & 0,97 & 0,2693 \\
\hline
\end{tabular}

für Shapiro-Wilk's W-Test $\boldsymbol{\alpha}=\mathbf{0 , 2 5}$

Tabelle 38: Kirchheim unter Teck - Statistisch deskriptive Daten der Histologiegruppen

\begin{tabular}{|l|l|l|r|r|r|r|r|r|r|r|}
\hline Gruppe & Variable & Einheit & Anzahl & $\begin{array}{c}\text { Mittel- } \\
\text { wert }\end{array}$ & Median & Min & Max & $\begin{array}{c}\text { Standard- } \\
\text { abweichung }\end{array}$ & $\begin{array}{c}\text { Normalverteilungstest } \\
\text { Shapiro-Wilk's W-Test } \\
\text { W }\end{array}$ \\
& & & & & & & & & & W \\
Histologie & $\mathrm{Ca} / \mathrm{P}$ & & 28 & 2,22 & 2,21 & 1,92 & 2,59 & 0,18 & 0,96 & 0,4030 \\
gut & $\mathrm{Sr} / \mathrm{Ca}$ & {$\left[\times 10^{-3}\right]$} & 28 & 0,44 & 0,45 & 0,32 & 0,58 & 0,06 & 0,97 & 0,6629 \\
& $\mathrm{Ba} / \mathrm{Ca}$ & {$\left[\times 10^{-3}\right]$} & 28 & 0,18 & 0,18 & 0,10 & 0,26 & 0,04 & 0,99 & 0,9619 \\
\hline Histologie & $\mathrm{Ca} / \mathrm{P}$ & & 73 & 2,18 & 2,17 & 1,50 & 2,81 & 0,21 & 0,98 & 0,4387 \\
schlecht & $\mathrm{Sr} / \mathrm{Ca}$ & {$\left[\times 10^{-3}\right]$} & 73 & 0,49 & 0,49 & 0,28 & 0,76 & 0,09 & 0,97 & 0,3392 \\
& $\mathrm{Ba} / \mathrm{Ca}$ & {$\left[\times 10^{-3}\right]$} & 73 & 0,25 & 0,25 & 0,14 & 0,44 & 0,06 & 0,96 & 0,0641 \\
\hline
\end{tabular}

für Shapiro-Wilk's W-Test $\boldsymbol{\alpha}=\mathbf{0 , 2 5}$

Tabelle 39: Wenigumstadt - Statistisch deskriptive Daten der Histologiegruppen

\begin{tabular}{|c|c|c|c|c|c|c|c|c|c|c|}
\hline \multirow[t]{2}{*}{ Gruppe } & \multirow[t]{2}{*}{ Variable } & \multirow[t]{2}{*}{ Einheit } & \multirow[t]{2}{*}{ Anzahl } & \multirow[t]{2}{*}{$\begin{array}{l}\text { Mittel- } \\
\text { wert }\end{array}$} & \multirow[t]{2}{*}{ Median } & \multirow[t]{2}{*}{ Min } & \multirow[t]{2}{*}{ Max } & \multirow[t]{2}{*}{$\begin{array}{c}\text { Standard- } \\
\text { abweichung }\end{array}$} & \multicolumn{2}{|c|}{$\begin{array}{l}\text { Normalverteilungstest } \\
\text { Shapiro-Wilk's W-Test }\end{array}$} \\
\hline & & & & & & & & & $\mathbf{w}$ & p \\
\hline \multirow{5}{*}{$\begin{array}{l}\text { Histologie } \\
\text { gut }\end{array}$} & Ätzen & [\%] & 29 & 9,3 & 9,5 & 5,8 & 14,2 & 2,0 & 0,98 & 0,7671 \\
\hline & Veraschen & [\%] & 29 & 30,3 & 30,5 & 26,1 & 32,4 & 1,5 & 0,93 & 0,0783 \\
\hline & $\mathrm{Ca} / \mathrm{P}$ & & 22 & 2,34 & 2,34 & 2,14 & 2,49 & 0,07 & 0,97 & 0,6485 \\
\hline & $\mathrm{Sr} / \mathrm{Ca}$ & {$\left[\times 10^{-3}\right]$} & 29 & 0,73 & 0,72 & 0,50 & 1,05 & 0,11 & 0,96 & 0,4615 \\
\hline & $\mathrm{Ba} / \mathrm{Ca}$ & {$\left[\times 10^{-3}\right]$} & 29 & 0,19 & 0,19 & 0,11 & 0,28 & 0,04 & 0,96 & 0,2887 \\
\hline \multirow{5}{*}{$\begin{array}{l}\text { Histologie } \\
\text { schlecht }\end{array}$} & Ätzen & & 57 & 12,1 & 12,2 & 6,2 & 17,8 & 2,5 & 0,98 & 0,8240 \\
\hline & Veraschen & & 57 & 2,7 & 2,7 & 2,2 & 3,3 & 2,9 & 0,94 & 0,0200 \\
\hline & $\mathrm{Ca} / \mathrm{P}$ & & 48 & 2,30 & 2,29 & 2,02 & 2,65 & 0,12 & 0,98 & 0,5909 \\
\hline & $\mathrm{Sr} / \mathrm{Ca}$ & {$\left[\times 10^{-3}\right]$} & 57 & 0,90 & 0,90 & 0,44 & 1,32 & 0,17 & 0,99 & 0,9552 \\
\hline & $\mathrm{Ba} / \mathrm{Ca}$ & {$\left[\times 10^{-3}\right]$} & 57 & 0,29 & 0,28 & 0,10 & 0,57 & 0,09 & 0,96 & 0,0875 \\
\hline
\end{tabular}

für Shapiro-Wilk's W-Test $\boldsymbol{\alpha}=\mathbf{0 , 2 5}$ 
Tabelle 40: Kirchheim am Ries - Statistisch deskriptive Daten der Histologiegruppen des unbehandelten Probensatz

\begin{tabular}{|c|c|c|c|c|c|c|c|c|c|c|}
\hline \multirow[t]{2}{*}{ Gruppe } & \multirow[t]{2}{*}{ Variable } & \multirow[t]{2}{*}{ Einheit } & \multirow[t]{2}{*}{ Anzahl } & \multirow[t]{2}{*}{$\begin{array}{c}\text { Mittel- } \\
\text { wert }\end{array}$} & \multirow[t]{2}{*}{ Median } & \multirow[t]{2}{*}{ Min } & \multirow[t]{2}{*}{ Max } & \multirow[t]{2}{*}{$\begin{array}{l}\text { Standard- } \\
\text { abweichung }\end{array}$} & \multicolumn{2}{|c|}{$\begin{array}{l}\text { Normalverteilungstest } \\
\text { Shapiro-Wilk's W-Test }\end{array}$} \\
\hline & & & & & & & & & $\mathbf{w}$ & p \\
\hline \multirow{5}{*}{$\begin{array}{c}\text { Histologie } \\
\text { gut }\end{array}$} & $\mathrm{Al} / \mathrm{Ca}$ & {$\left[\times 10^{-3}\right]$} & 18 & 0,29 & 0,26 & 0,10 & 1,00 & 0,20 & 0,72 & 0,0001 \\
\hline & $\mathrm{Ba} / \mathrm{Ca}$ & {$\left[\times 10^{-3}\right]$} & 18 & 0,11 & 0,11 & 0,06 & 0,18 & 0,03 & 0,97 & 0,7788 \\
\hline & $\mathrm{Mg} / \mathrm{Ca}$ & {$\left[\times 10^{-3}\right]$} & 18 & 3,07 & 2,79 & 2,22 & 4,13 & 0,62 & 0,86 & 0,0095 \\
\hline & $\mathrm{Ca} / \mathrm{P}$ & & 18 & 2,34 & 2,34 & 2,27 & 2,39 & 0,03 & 0,96 & 0,6372 \\
\hline & $\mathrm{Sr} / \mathrm{Ca}$ & {$\left[\times 10^{-3}\right]$} & 18 & 0,34 & 0,34 & 0,24 & 0,43 & 0,05 & 0,98 & 0,9253 \\
\hline \multirow{5}{*}{$\begin{array}{l}\text { Histologie } \\
\text { schlecht }\end{array}$} & $\mathrm{Al} / \mathrm{Ca}$ & {$\left[\times 10^{-3}\right]$} & 30 & 0,56 & 0,44 & 0,04 & 1,43 & 0,41 & 0,87 & 0,0016 \\
\hline & $\mathrm{Ba} / \mathrm{Ca}$ & {$\left[\times 10^{-3}\right]$} & 30 & 0,20 & 0,19 & 0,11 & 0,40 & 0,07 & 0,91 & 0,0138 \\
\hline & $\mathrm{Mg} / \mathrm{Ca}$ & {$\left[\times 10^{-3}\right]$} & 30 & 2,02 & 2,01 & 1,58 & 2,65 & 0,25 & 0,98 & 0,7417 \\
\hline & $\mathrm{Ca} / \mathrm{P}$ & & 30 & 2,33 & 2,33 & 2,23 & 2,45 & 0,04 & 0,98 & 0,8476 \\
\hline & $\mathrm{Sr} / \mathrm{Ca}$ & {$\left[\times 10^{-3}\right]$} & 30 & 0,46 & 0,44 & 0,34 & 0,63 & 0,08 & 0,93 & 0,0458 \\
\hline
\end{tabular}

für Shapiro-Wilk's W-Test $\boldsymbol{\alpha}=\mathbf{0 , 2 5}$

Tabelle 41: Kirchheim am Ries - Statistisch deskriptive Daten der Histologiegruppen des geätzten Probensatzes

\begin{tabular}{|c|c|c|c|c|c|c|c|c|c|c|}
\hline \multirow[t]{2}{*}{ Gruppe } & \multirow[t]{2}{*}{ Variable } & \multirow[t]{2}{*}{ Einheit } & \multirow[t]{2}{*}{ Anzahl } & \multirow[t]{2}{*}{$\begin{array}{l}\text { Mittel- } \\
\text { wert }\end{array}$} & \multirow[t]{2}{*}{ Median } & \multirow[t]{2}{*}{ Min } & \multirow[t]{2}{*}{ Max } & \multirow[t]{2}{*}{$\begin{array}{l}\text { Standard- } \\
\text { abweichung }\end{array}$} & \multicolumn{2}{|c|}{$\begin{array}{l}\text { Normalverteilungstest } \\
\text { Shapiro-Wilk's W-Test }\end{array}$} \\
\hline & & & & & & & & & $\mathbf{w}$ & p \\
\hline \multirow{6}{*}{$\begin{array}{l}\text { Histologie } \\
\text { gut }\end{array}$} & Ätzen & [\%] & 18 & 18,0 & 18,9 & 12,7 & 21,8 & 3,3 & 0,87 & 0,0180 \\
\hline & Veraschen & {$[\%]$} & 18 & 28,4 & 28,6 & 26,3 & 31,4 & 1,4 & 0,94 & 0,2576 \\
\hline & $\mathrm{Al} / \mathrm{Ca}$ & {$\left[\times 10^{-3}\right]$} & 18 & 0,13 & 0,08 & 0,00 & 0,69 & 0,16 & 0,69 & 0,0000 \\
\hline & $\mathrm{Ba} / \mathrm{Ca}$ & {$\left[\times 10^{-3}\right]$} & 18 & 0,11 & 0,11 & 0,06 & 0,18 & 0,03 & 0,96 & 0,6109 \\
\hline & $\mathrm{Mg} / \mathrm{Ca}$ & {$\left[\times 10^{-3}\right]$} & 18 & 3,17 & 2,87 & 2,35 & 4,44 & 0,69 & 0,89 & 0,0355 \\
\hline & $\mathrm{Sr} / \mathrm{Ca}$ & {$\left[\times 10^{-3}\right]$} & 18 & 0,34 & 0,33 & 0,24 & 0,42 & 0,05 & 0,94 & 0,3536 \\
\hline \multirow{6}{*}{$\begin{array}{l}\text { Histologie } \\
\text { schlecht }\end{array}$} & Ätzen & {$[\%]$} & 30 & 25,1 & 24,1 & 19,4 & 44,6 & 5,2 & 0,79 & 0,0000 \\
\hline & Veraschen & [\%] & 30 & 15,1 & 14,2 & 12,0 & 20,8 & 2,3 & 0,93 & 0,0592 \\
\hline & $\mathrm{Al} / \mathrm{Ca}$ & {$\left[\times 10^{-3}\right]$} & 30 & 0,52 & 0,32 & 0,00 & 1,82 & 0,51 & 0,85 & 0,0004 \\
\hline & $\mathrm{Ba} / \mathrm{Ca}$ & {$\left[\times 10^{-3}\right]$} & 30 & 0,19 & 0,17 & 0,10 & 0,40 & 0,07 & 0,90 & 0,0086 \\
\hline & $\mathrm{Mg} / \mathrm{Ca}$ & {$\left[\times 10^{-3}\right]$} & 30 & 1,88 & 1,85 & 1,29 & 2,66 & 0,28 & 0,98 & 0,8146 \\
\hline & $\mathrm{Sr} / \mathrm{Ca}$ & {$\left[\times 10^{-3}\right]$} & 30 & 0,45 & 0,43 & 0,32 & 0,62 & 0,09 & 0,94 & 0,1106 \\
\hline
\end{tabular}

für Shapiro-Wilk's W-Test $\boldsymbol{\alpha}=\mathbf{0 , 2 5}$

Tabelle 42: Kirchheim am Ries - Statistisch deskriptive Daten der Histologiegruppen des oberflächenbehandelten Probensatz

\begin{tabular}{|c|c|c|c|c|c|c|c|c|c|c|}
\hline \multirow[t]{2}{*}{ Gruppe } & \multirow[t]{2}{*}{ Variable } & \multirow[t]{2}{*}{ Einheit } & \multirow[t]{2}{*}{ Anzahl } & \multirow[t]{2}{*}{$\begin{array}{c}\text { Mittel- } \\
\text { wert }\end{array}$} & \multirow[t]{2}{*}{ Median } & \multirow[t]{2}{*}{ Min } & \multirow[t]{2}{*}{ Max } & \multirow[t]{2}{*}{$\begin{array}{c}\text { Standard- } \\
\text { abweichung }\end{array}$} & \multicolumn{2}{|c|}{$\begin{array}{l}\text { Normalverteilungstest } \\
\text { Shapiro-Wilk's W-Test }\end{array}$} \\
\hline & & & & & & & & & $\mathbf{w}$ & $p$ \\
\hline \multirow{4}{*}{$\begin{array}{c}\text { Histologie } \\
\text { gut }\end{array}$} & $\mathrm{Al} / \mathrm{Ca}$ & {$\left[\times 10^{-3}\right]$} & 18 & 0,08 & 0,02 & 0,00 & 0,59 & 0,14 & 0,61 & 0,0000 \\
\hline & $\mathrm{Ba} / \mathrm{Ca}$ & {$\left[\times 10^{-3}\right]$} & 18 & 0,11 & 0,11 & 0,06 & 0,19 & 0,03 & 0,93 & 0,1714 \\
\hline & $\mathrm{Mg} / \mathrm{Ca}$ & {$\left[\times 10^{-3}\right]$} & 18 & 3,39 & 3,13 & 2,59 & 4,77 & 0,70 & 0,90 & 0,0696 \\
\hline & $\mathrm{Sr} / \mathrm{Ca}$ & {$\left[\times 10^{-3}\right]$} & 18 & 0,34 & 0,34 & 0,23 & 0,40 & 0,04 & 0,95 & 0,3619 \\
\hline \multirow{4}{*}{$\begin{array}{l}\text { Histologie } \\
\text { schlecht }\end{array}$} & $\mathrm{Al} / \mathrm{Ca}$ & {$\left[\times 10^{-3}\right]$} & 30 & 0,33 & 0,18 & 0,00 & 1,15 & 0,32 & 0,87 & 0,0012 \\
\hline & $\mathrm{Ba} / \mathrm{Ca}$ & {$\left[\times 10^{-3}\right]$} & 30 & 0,20 & 0,18 & 0,11 & 0,40 & 0,07 & 0,91 & 0,0203 \\
\hline & $\mathrm{Mg} / \mathrm{Ca}$ & {$\left[\times 10^{-3}\right]$} & 30 & 2,10 & 2,08 & 1,53 & 2,90 & 0,31 & 0,97 & 0,6744 \\
\hline & $\mathrm{Sr} / \mathrm{Ca}$ & {$\left[\times 10^{-3}\right]$} & 30 & 0,47 & 0,46 & 0,35 & 0,64 & 0,09 & 0,93 & 0,0704 \\
\hline
\end{tabular}

für Shapiro-Wilk's W-Test $\boldsymbol{\alpha}=\mathbf{0 , 2 5}$ 
Tabelle 43: Kirchheim am Ries - Statistisch deskriptive Daten der Histologiegruppen des Probensatzes, der oberflächenbehandelt und geätzt wurde

\begin{tabular}{|c|l|l|l|r|r|r|r|r|r|r|}
\hline Gruppe & Variable & Einheit & Anzahl & $\begin{array}{c}\text { Mittel- } \\
\text { wert }\end{array}$ & Median & Min & Max & $\begin{array}{c}\text { Standard- } \\
\text { abweichung }\end{array}$ & $\begin{array}{c}\text { Normalverteilungstest } \\
\text { Shapiro-Wilk's W-Test } \\
\mathbf{~ W}\end{array}$ \\
& & & & & & & & & & $\mathbf{w}$ \\
\hline Histologie & $\mathrm{Al} / \mathrm{Ca}$ & {$\left[\times 10^{-3}\right]$} & 18 & 0,08 & 0,04 & 0,00 & 0,65 & 0,15 & 0,53 & 0,0000 \\
gut & $\mathrm{Ba} / \mathrm{Ca}$ & {$\left[\times 10^{-3}\right]$} & 18 & 0,10 & 0,10 & 0,06 & 0,18 & 0,03 & 0,95 & 0,4958 \\
& $\mathrm{Mg} / \mathrm{Ca}$ & {$\left[\times 10^{-3}\right]$} & 18 & 3,26 & 3,07 & 2,47 & 4,81 & 0,73 & 0,89 & 0,0469 \\
& $\mathrm{Sr} / \mathrm{Ca}$ & {$\left[\times 10^{-3}\right]$} & 18 & 0,32 & 0,32 & 0,23 & 0,38 & 0,04 & 0,96 & 0,6869 \\
\hline Histologie & $\mathrm{Al} / \mathrm{Ca}$ & {$\left[\times 10^{-3}\right]$} & 30 & 0,38 & 0,20 & 0,00 & 1,24 & 0,37 & 0,85 & 0,0003 \\
schlecht & $\mathrm{Ba} / \mathrm{Ca}$ & {$\left[\times 10^{-3}\right]$} & 30 & 0,18 & 0,16 & 0,10 & 0,36 & 0,06 & 0,89 & 0,0039 \\
& $\mathrm{Mg} / \mathrm{Ca}$ & {$\left[\times 10^{-3}\right]$} & 30 & 1,80 & 1,75 & 1,26 & 2,59 & 0,30 & 0,91 & 0,0202 \\
& $\mathrm{Sr} / \mathrm{Ca}$ & {$\left[\times 10^{-3}\right]$} & 30 & 0,43 & 0,42 & 0,30 & 0,63 & 0,09 & 0,94 & 0,1177 \\
\hline
\end{tabular}

für Shapiro-Wilk's W-Test $\boldsymbol{\alpha}=\mathbf{0 , 2 5}$

Tabelle 44: EPMA-Analysen - Statistisch deskriptive Daten für "native" und veränderte Mineralareale innerhalb des Kompaktaquerschnittes der Knochenprobe KR 261 von Kirchheim am Ries

\begin{tabular}{|c|l|r|l|r|r|r|r|r|r|r|}
\hline Gruppe & Variable & Einheit & Anzahl & $\begin{array}{c}\text { Mittel- } \\
\text { wert }\end{array}$ & Median & Min & Max & $\begin{array}{c}\text { Standard- } \\
\text { abweichung }\end{array}$ & $\begin{array}{c}\text { Normalverteilungstest } \\
\text { Shapiro-Wilk's W-Test } \\
\text { W }\end{array}$ \\
& & & & & & & & & W & $\mathbf{p}$ \\
\hline Mineral & $\mathrm{Na} / \mathrm{Ca}$ & {$\left[\times 10^{-3}\right]$} & 49 & 10,39 & 10,55 & 3,72 & 13,18 & 1,70 & 0,93 & 0,0082 \\
& $\mathrm{Ca} / \mathrm{P}$ & {$\left[\times 10^{-3}\right]$} & 49 & 2,26 & 2,25 & 2,13 & 2,44 & 0,05 & 0,89 & 0,0002 \\
& $\mathrm{Mg} / \mathrm{Ca}$ & {$\left[\times 10^{-3}\right]$} & 49 & 2,84 & 2,79 & 1,81 & 4,94 & 0,61 & 0,96 & 0,1456 \\
& $\mathrm{~S} / \mathrm{Ca}$ & {$\left[\times 10^{-3}\right]$} & 49 & 4,10 & 4,21 & 1,60 & 5,24 & 0,66 & 0,95 & 0,0419 \\
\hline Mineral & $\mathrm{Na} / \mathrm{Ca}$ & {$\left[\times 10^{-3}\right]$} & 27 & 2,10 & 2,02 & 0,00 & 5,58 & 1,71 & 0,91 & 0,0302 \\
verändert & $\mathrm{Ca} / \mathrm{P}$ & {$\left[\times 10^{-3}\right]$} & 27 & 2,25 & 2,25 & 2,15 & 2,35 & 0,05 & 0,97 & 0,7227 \\
& $\mathrm{Mg} / \mathrm{Ca}$ & {$\left[\times 10^{-3}\right]$} & 27 & 1,36 & 1,30 & 0,00 & 3,80 & 0,70 & 0,89 & 0,0091 \\
& $\mathrm{~S} / \mathrm{Ca}$ & {$\left[\times 10^{-3}\right]$} & 27 & 2,01 & 1,93 & 1,08 & 3,63 & 0,67 & 0,91 & 0,0221 \\
& $\mathrm{Na} / \mathrm{Ca}$ & {$\left[\times 10^{-3}\right]$} & 27 & 2,10 & 2,02 & 0,00 & 5,58 & 1,71 & 0,91 & 0,0302 \\
\hline
\end{tabular}

für Shapiro-Wilk's W-Test $\boldsymbol{\alpha}=\mathbf{0 , 2 5}$ 


\section{Danksagung}

Herrn Prof. Dr. Bernd Herrmann gilt mein Dank für die Bereitstellung des Themas und des Arbeitsplatzes sowie für die Nachsicht meinen "wenn und abers" gegenüber; vor allem aber für die Geduld in den letzten Wochen.

Ebenfalls bin ich PD Dr. Holger Schutkowski, der das Projekt beantragt, Daten zur Verfügung gestellt und mich betreut hat, zu großem Dank verpflichtet.

Bedanken möchte ich mich bei Frau Dr. Susanne Hummel für ihre Unterstützung in Form von Gesprächen, Diskussionen und Anregungen.

Für Übernahme des Korreferats und die Ratschläge hinsichtlich der statistischen Auswertungen danke ich Prof. Dr. Hartmut Rothe.

Herrn Dr. Joachim Wahl vom Landesdenkmalamt Baden-Württemberg danke ich für die Überlassung des Skelettmaterials.

Für die umfangreiche Unterstützung bei den Analysen, die weit über das übliche Maß hinausging, möchte ich mich bei Dr. Klaus Simon, Dr. Andreas Kronz und Erwin Schiffczyk aus der Geochemie des Göttinger Zentrums Geowissenschaften bedanken. Sie standen auch nach Abschluß der Analysen jederzeit für (viele) Nachfragen zur Verfügung und waren mir damit eine große Hilfe.

Ich danke Prof. Dr. Edgar Brunner, der sich die Zeit genommen hat, meine statistischen Probleme mit mir $\mathrm{zu}$ besprechen, und Carola Werner für die Realisierung der SAS-Berechnungen. In diesem Zusammenhang auch Stefan Suchi Dank für seine Beratung.

Ein ganz besonderer Dank gilt Sabine Becker, die mich in das Labor eingeführt und über die Jahre begleitet hat, ebenso Nicole Weber, Annegret Becker und Birgit Zeike für ihre vielfältige Mitarbeit bei Probenvorbereitungen und Analysen. Andrea Smoczyk danke ichfür ihre Mühe bei den Histologien. Ed George hat mich mit persönlichem (beruhigendem) Rat und technischer Hilfe jederzeit unterstützt. Dafür möchte ich mich ganz herzlich bei ihm bedanken. Für die Durchführung der phototechnischen Arbeiten danke ich Sibylle Hourticolon. Frau Tavakolian danke ich für ihre Umsicht angesichts meiner finanztechnischen Unzulänglichkeiten.

Ein Dankeschön meinen studentischen Hilfskräften im Labor: Nele Schwarz, Annette Dreschler, Oliver Beykirch.

Ich danke Freunden und Kollegen innerhalb und außerhalb des Institutes, die mich über die Jahre begleitet, unterstützt und nicht zuletzt meine Launen und meinen Pessimismus ertragen haben, auch wenn sie hier nicht namentlich genannt werden.

Zweien, Svenja Weise und Julia Gerstenberger, sei aber zusätzlich für die schier unermeßliche Geduld der letzten Wochen gedankt.

Das Projekt wurde aus Mitteln der deutschen Forschungsgemeinschaft finanziert.

Darüberhinaus bedanke ich mich bei meiner Familie, besonders bei meinen Eltern, für die vielfältige Unterstützung während der gesamten Studienzeit. 


\section{Lebenslauf}

Alexander Fabig

Staatsangehörigkeit

deutsch

01.03 .1968

$1974-1984$

$1984-1986$

geboren in Mühlhausen

Besuch der Zehnklassigen Allgemeinbildenden Polytechnischen Oberschule in Birkungen

Besuch der Erweiterten Allgemeinbildenden Polytechnischen Oberschule in Leinefelde

04.07 .1986

$1986-1987$

$1987-1988$

$1988-1990$

$1990-1998$

15.10 .1992

Absolvierung des Abiturs

Tätigkeit als Werkstoffprüfer in den Eichsfelder Zementwerken Deuna

Wehrdienst

Physikstudium an der Technischen Hochschule „Carl Schorlemmer" in Merseburg.

Biologiestudium an der Georg-August-Universität Göttingen

Biologie-Diplomvorprüfung in den Fächern Zoologie, Genetik, Anorganische Chemie und Physikalische Chemie

28.06 .1996

mündliche Prüfungen zum Diplom in Biologie in den Fächern Anthropologie, Zoologie und Ethnologie

$1996-1998$

30.03 .1998

$30.03 .1998-31.03 .1999$

seit 01.04.1999

Diplomarbeit in der Abteilung Historische Anthropologie und Humanökologie in Göttingen mit dem Titel „Die Rekonstruktion der Ernährungsgrundlage als Möglichkeit der Differenzierung zwischen berufs- und ernährungsbedingten Elementeinträgen in das Skelett am Beispiel einer Bergbaubevölkerung. Eine methodenorientierte Untersuchung an Knochenproben und Nahrungsmitten"

Diplom in Biologie

Anstellung als wissenschaftliche Hilfskraft in der Abteilung für Historische Anthropologie und Humanökologie, Göttingen

Wissenschaftlicher Angestellter in der Abteilung für Historische Anthropologie und Humanökologie und Beginn des Promotionsvorhabens „Spurenelementuntersuchungen an bodengelagertem Skelettmaterial. Validitätserwägungen im Kontext diagenetisch bedingter Konzentrationsänderungen des Knochenminerals“ im Rahmen des DFG-Projektes „Biokulturelle Variabilität der Ressourcennutzung und Nahrungsnetzanalyse in historischen Bevölkerungen“" 